\title{
IMPACT OF PROTESTANT SPIRITUALITY AND RELIGIOUS COMMUNITY ON THE WELL-BEING OF PEOPLE IN HONG KONG
}

\author{
Chi Wai (Eddie) Ng \\ Bachelor of Social Science (Psychology) \\ Master of Arts (Religious Studies)
}

Thesis submitted in fulfilment of the requirements of the degree of Doctor of Philosophy

School of Social Sciences and Psychology

Faculty of Arts, Education and Human Development

Victoria University

September 2009 


\begin{abstract}
While the literature demonstrates that the relationship between religion and wellbeing is generally positive, detailed information about the context where the religion is based is rarely given. Drawing on multi-dimensional perspectives of spirituality, the present study regards spirituality as an integral dimension of a person's religious beliefs, practices and experiences within a given context. The manifestation of spirituality can be viewed from personal, interpersonal and social dimensions. The primary objective of the study is to examine whether, how and why the spirituality and religious community of Protestant tradition can affect the well-being of people in the context of Hong Kong.

In the first phase of the study, 324 Chinese from four Protestant communities, representing four major kinds of Protestant spirituality, and one non-religious comparison group in Hong Kong were recruited. MANOVA results showed that religious community exerted a significant impact on people's spirituality and sense of community, which enhance their well-being - manifested in life satisfaction, social trust and civic participation. The different religious contexts exhibited different dimensions of spirituality and well-being, suggesting that the impact is not only direct but also dependent on and intertwined with the religious setting. Path analyses demonstrated the complex relationship between spirituality and well-being variables. While some of the spirituality-related variables may directly predict civic participation, the relationship between such variables and life satisfaction and social trust are mainly mediated through mental health and sense of community.

The second phase of the study incorporated a field study (participant observation, interview and documentary analysis). Activity setting analysis was used as the interpretive framework, to unpack the elements of the various religious settings and to identify the present and absent conditions that help explain the variety and diversity of well-being outcomes manifested in the survey section. The data showed that immediate religious settings affect people's emotions, cognitions and behavioural patterns, through relational, ideational and even material aspects of the social environment, which finally lead to various kinds of well-being. Results also demonstrate that not only may the different religious settings enhance different kinds of well-being, but that they also provide different pathways to enhance a certain kind of well-being. These findings
\end{abstract}


suggest that religious impacts on people's well-being are diverse and intricate. The present study thus confirms the important role of religion in people's well-being, but further signifies the intricate relationship between the various dimensions of religion and well-being. Moreover, when studying the effects of religion, both the subjective and the community context of a person's spirituality cannot be overlooked when drawing any conclusion on the costs and benefits of religion. 


\section{Declaration}

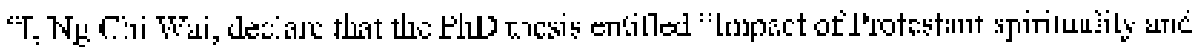

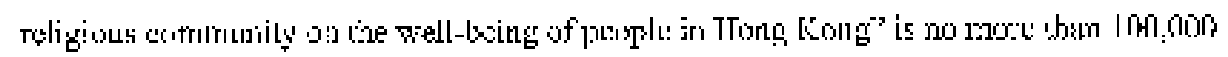

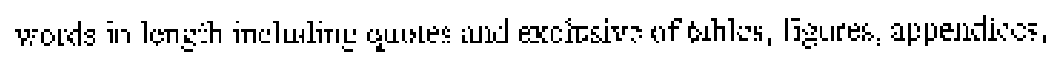

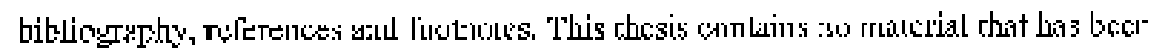

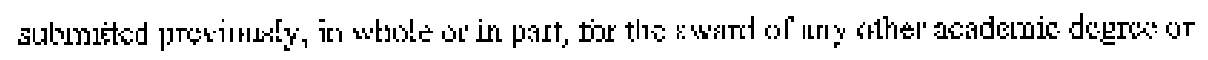

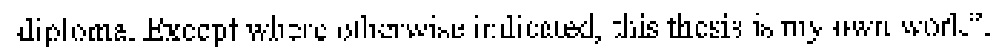

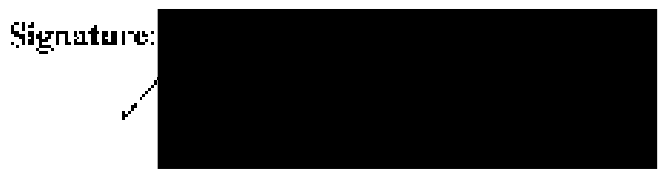

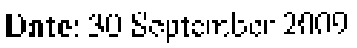




\section{Acknowledgements}

Furthering my study overseas is both my dream and the soul journey towards realizing my psychology ideal in the real world. In this journey I am so privileged to have many kind persons to support me, without whom, I cannot finish this journey.

I should thank Heather Gridley without whose tender guidance and ever-ready support in the beginning stage I think I would not start my journey in pursuing my dream study in community psychology and in Australia.

With no doubt, I am deeply indebted to Adrian Fisher, my supervisor, without his incisive mind, intellectual and all the tangible support I cannot pursue and finish this $\mathrm{PhD}$ degree. I have not only learned a lot from the supervision and criticism, but also enjoy our sharing about the university, community psychology and career or other professional development. But most importantly, his insistently saying "You can do it" often become my driving force especially when I was also wondering about my ability. Having a person who has confidence in you more than yourself is really my privilege and the blessing from the heaven.

I would also thank my Victoria University community: Associate Professor Michele Grossman and Professor Jill Asbury - who gave their support in my application of the IPRS scholarship; Lesley Birch - for her meticulous and laborious administrative support in my scholarship matters; Angela Rojter - for her patient and excellent language support, the office of Postgraduate Research staff - for their inspiring and stimulating research support; Chris Sonn, Wally Karnilowicz, Andrew Jago and Delwyn Goodrick for their stimulation and valuable suggestions in my study; the community psychology community - for their company and the joy we share along the years.

I would also like to thank the people in the Hong Kong community, in which, Dr. Lam, C. W. - for his support to my pursuing the PhD; Dr. Harry C. H. Hui, Dr. Cindy Lam, Prof. Shek. T. L., Prof. Bond, M. - in providing suggestions and advice in the study process; Dr. Lai Chi Tim, Dr. Simon Kwan, Dr. Ying Fuk Tsang - for allowing me to sit in their classes related to my study; Sylvia So, Henry Li, Yam Chi Keung - for their valuable feedback to my study in different stages; Kit Wong, Jeffrey Liu, Christine Au, 
Joe Yau, Peter Lok, Agnes Leung, and the STMC fellows brothers and sisters - for their help in the liaising process, piloting and data collection phases of the study.

Certainly, I would also give thanks to the four participating churches and the social centre and all the participants who took part in either the survey or interview. Your participation in the study not only confirms my thought about the religion and well-being, but also broadens my view about the intricacy of relationship between the religion and well-being.

Definitely, I would also like to express my deep gratitude to my parents who are selflessly and willing to provide whatever support they can to their child and gave me freedom and opportunities to pursue my dream. Thanks also to my brothers Conrad and Zeno for their concern and regards from time to time. Undoubtedly, I would also like to thank my wife, Ka Yee, for supporting me throughout the whole journey. Her company and support, whether tangible, emotional or spiritual, means a lot to me and her insightful, if not humorous, comments and suggestions are always a source of stimulation and fresh breath in this long, if not lonely, journey.

Finally, I would also like to thank my heavenly Father who keeps calling and sustaining me to attain the goal that I haven’t dreamed of before. To Him I offer my little contribution and my great honour.

\section{GOD MAKE MY LIFE A LITTLE LIGHT}

God make my life a little light, Within the world to glow;

A little flame that burneth bright, Wherever I may go.

God make my life a little flower, That giveth joy to all, Content to bloom in native bower, Although the place be small.

God make my life a little song That comforteth the sad ;

That helpeth others to be strong, And make the singer glad.

God make my life a little staff Whereon the weak may rest, That so what health and strength I have May serve my neighbours best.

God make my life a little hymn Of tenderness and praise,

Of faith that never waxeth dim, In all His wondrous ways.

(Matilda Betham-Edwards) 


\section{Table of Contents}

\section{Chapter 1}

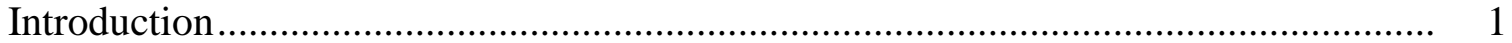

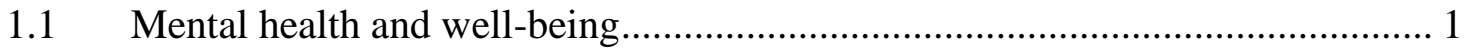

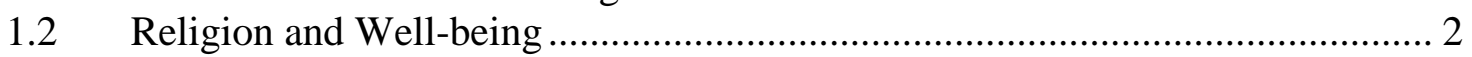

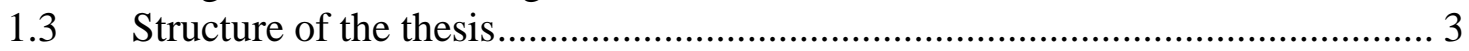

Chapter 2

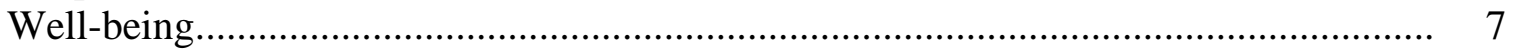

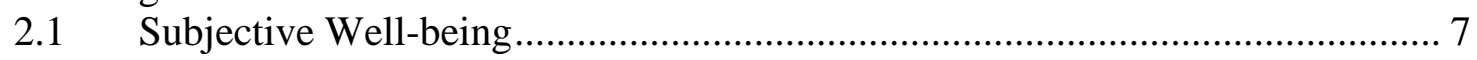

2.1.1 The components of subjective well-being …......................................... 7

2.1.2 Positive Psychology Movement............................................................ 9

2.1.3 Correlates of subjective well-being …................................................... 10

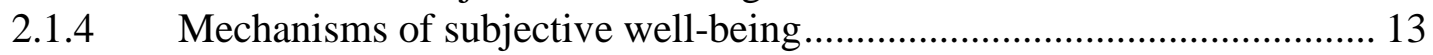

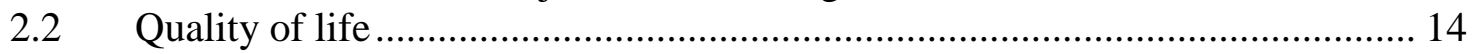

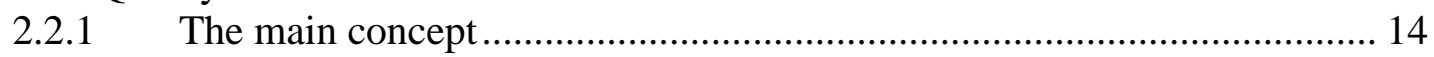

2.2.2 Quality of Life and Subjective Well-being ........................................... 17

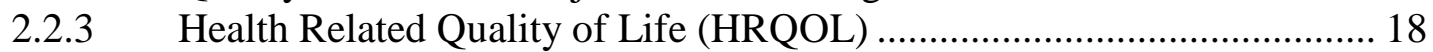

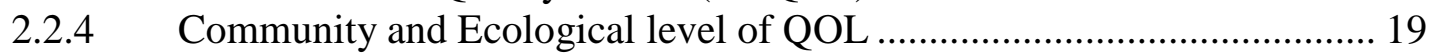

2.3 Multi-Level approach to well-being ........................................................... 20

2.3.1 The major proposition ..................................................................... 20

2.3.2 Comparison between different conceptualizations of well-being............. 21

2.3.3 Why multi-level understanding of well-being is important ...................... 22

2.4 A model of well-being ......................................................................... 24

Chapter 3

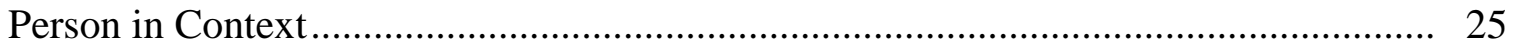

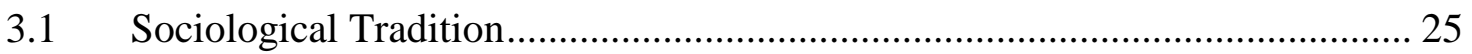

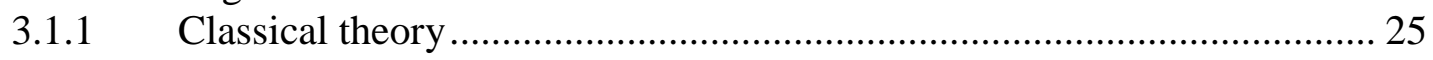

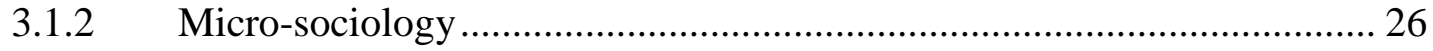

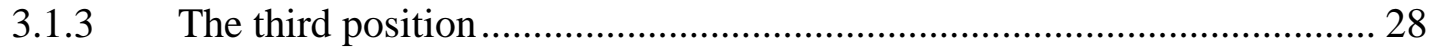

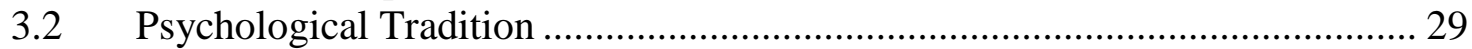

3.2.1 Barker’s Behaviour Setting Approach .................................................. 29

3.2.2 Bronfenbrenner's ecological approach ................................................ 31

3.2.3 Sarason's Psychological Sense of Community ...................................... 32

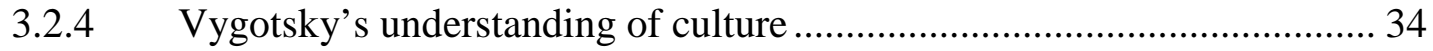

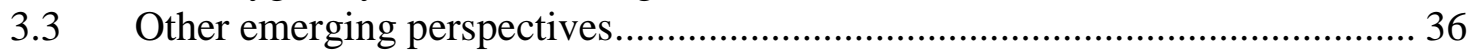

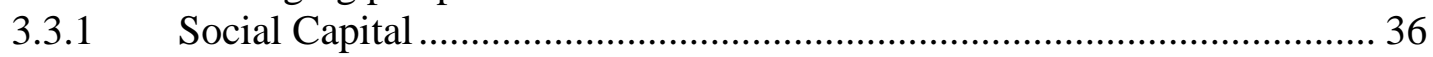

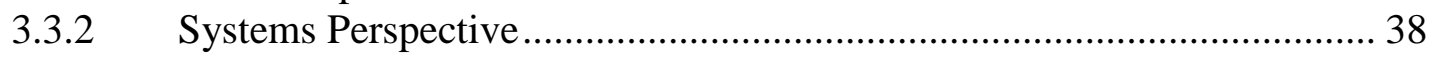

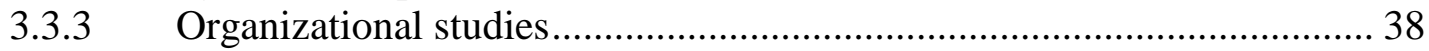

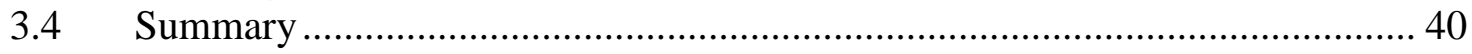

\section{Chapter 4}

The Conceptualization of Religion ..................................................................... 43

4.1 The Insider-Outsider Debate...................................................................... 43 


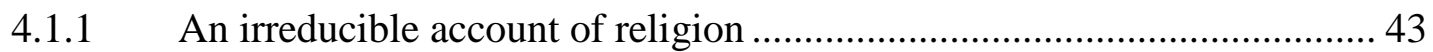

4.1.2 In defence of the reducible account of religion ......................................... 44

4.1.3 Agnostic stance ............................................................................... 44

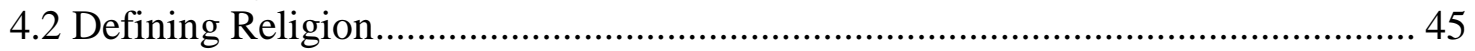

4.2.1 Spirituality and religion ....................................................................... 45

4.2.2 Phenomenological tradition ................................................................... 46

4.2.3 Psychological tradition - Religion as subjective religiosity ...................... 46

4.2.4 Sociological tradition - Religion as groups or organizations .................... 48

4.2.5 Anthropological tradition - Religion as a cultural system......................... 50

4.3 An integrated Approach......................................................................................... 51

Chapter 5

Religion and Well-being ...................................................................................... 55

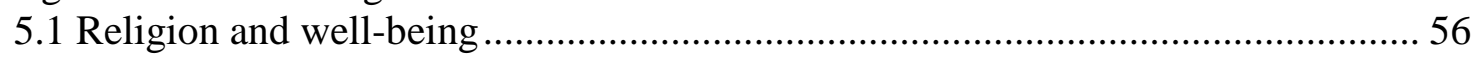

5.1.1 Religion and Individual Well-being.......................................................... 56

5.1.2 Religion and Interpersonal Well-being ..................................................... 57

5.1.3 Religion and Collective Well-being.......................................................... 58

5.1.4 Religion and the building of community .................................................. 60

5.2 The mechanism underlying religion and well-being ............................................. 61

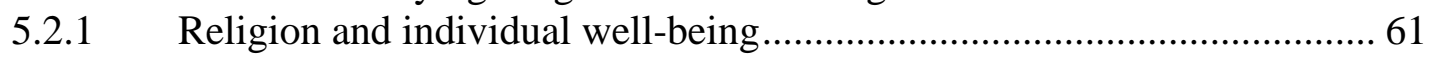

5.2.2 Religion and interpersonal and collective well-being.................................63 63

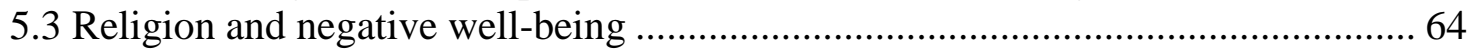

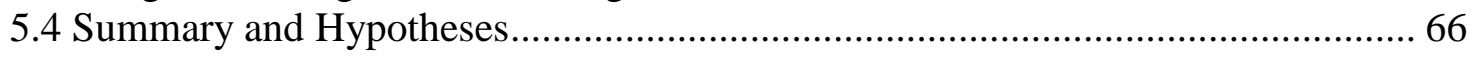

\section{Chapter 6}

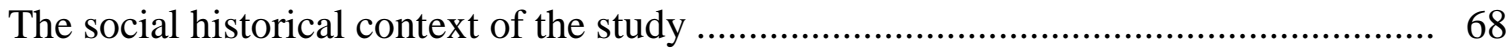

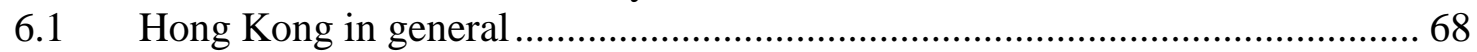

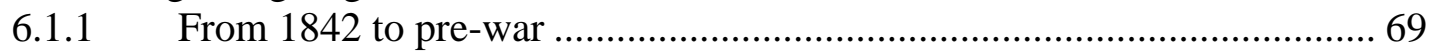

6.1.2 From post-war to the 1980s ............................................................................. 70

6.1.3 The return to Chinese sovereignty in 1997 .................................................... 71

6.2 Protestant Christianity in Hong Kong ......................................................... 72

6.2.1 From 1842 to pre-war .................................................................... 72

6.2.2 From post-war to the 1980s ........................................................................... 73

6.2.3 The return to Chinese sovereignty in 1997 ................................................... 75

6.3 The five settings in the present study ........................................................ 76

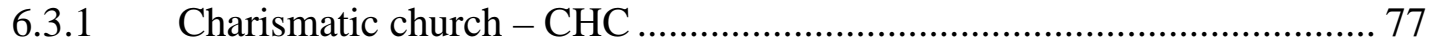

6.3.2 Social concern church - SCC …………………..................................... 78

6.3.3 Fundamentalist church - FDC ............................................................ 79

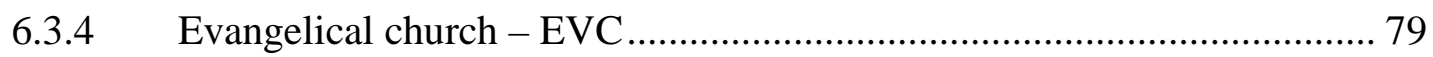

6.3.5 The comparison group - Social centre...................................................... 80

Chapter 7

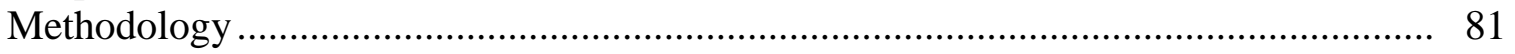

7.1 The rationale of using Mixed Method ……………................................................ 81

7.1.1 The limitation of conventional approach .................................................. 81

7.1.2 The contribution of qualitative research approach..................................... 82 
7.1.3 The importance of mixed methods in the present study ............................. 84

7.2 The procedure and structure of the present study ………….............................. 87

Chapter 8

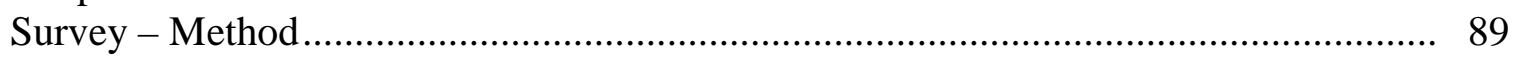

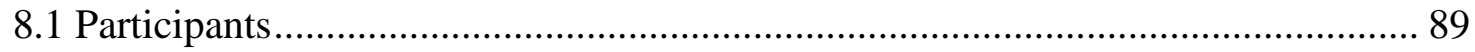

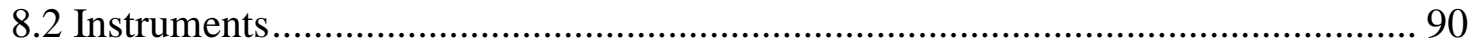

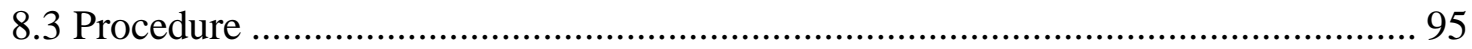

Chapter 9

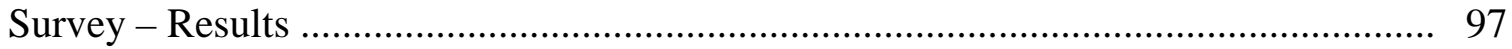

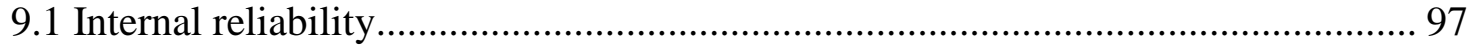

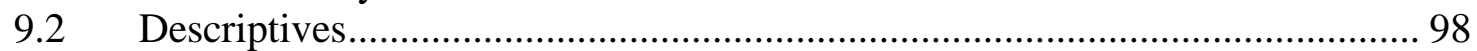

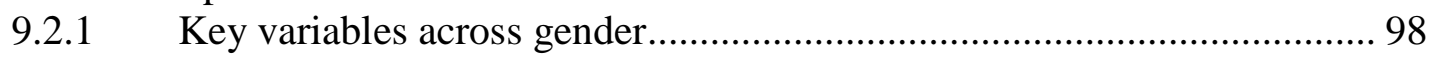

9.2.2 Education and income across settings............................................................. 100

9.2.3 Religious practices across settings.............................................................. 101

9.2.4 Participation in voluntary organizations across settings ........................... 103

9.2.5 Participation in church activities across settings ...................................... 104

9.2.6 Participants' personal identity across settings ......................................... 105

9.2.7 Congregational identity perceived by participants across settings .......... 106

9.3 The influence of spiritual context ......................................................................... 107

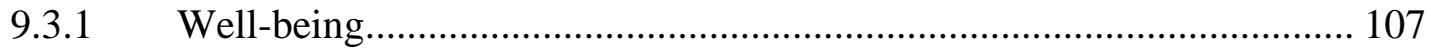

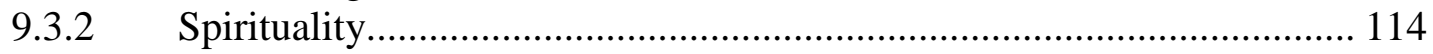

9.3.3 Sense of community and social belief .................................................. 117

9.4 Underlying patterns between groups ............................................................. 120

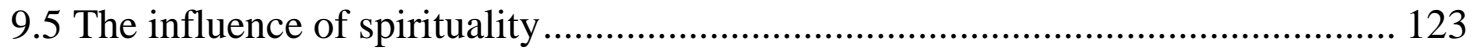

9.5.1 Relationship between Spirituality and life satisfaction............................ 124

9.5.2 Relation between Spirituality and Social Trust ........................................ 128

9.5.3 Relationship between Spirituality and Civic participation .............................. 130

9.5.4 Path Analysis using AMOS ...................................................................... 134

\section{Chapter 10}

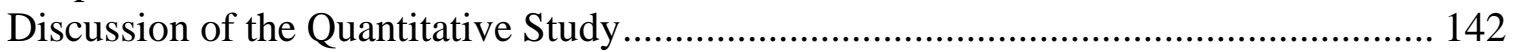

10.1 Comparisons between settings ........................................................................... 142

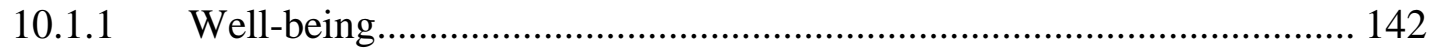

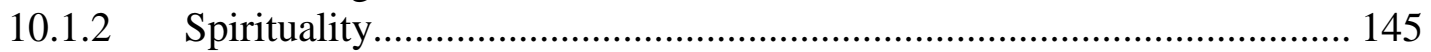

10.1.3 Sense of community and social belief .................................................. 147

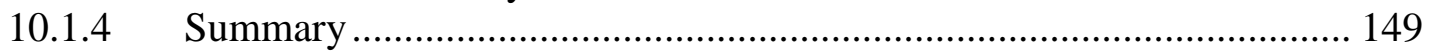

10.2 Dimensions differentiating the settings .................................................... 150

10.2.1 Taxonomy of settings....................................................................... 150

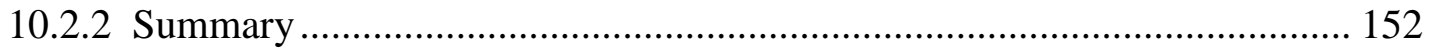

10.3 The association between spirituality on well-being.......................................... 153

10.3.1 Prediction of Life satisfaction............................................................ 153

10.3.2 Prediction of Social Trust .................................................................... 156

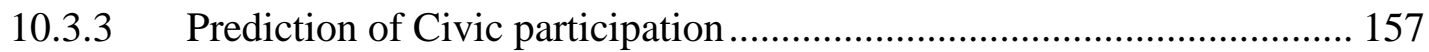

10.3.4 Overall Well-Being ...................................................................................... 159 


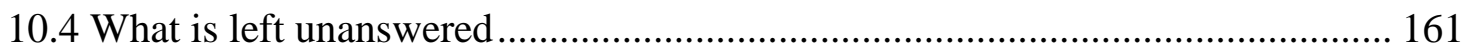

Chapter 11

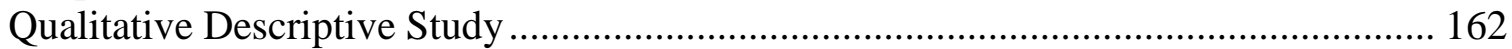

11.1 Activity Setting Analysis ................................................................................ 162

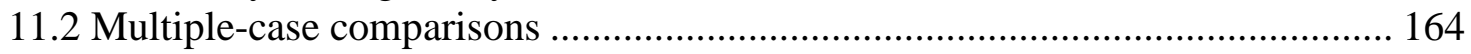

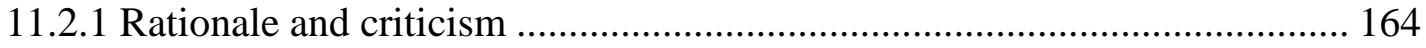

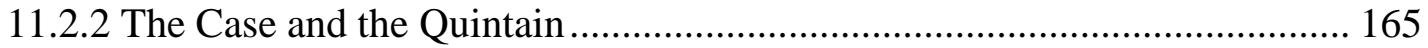

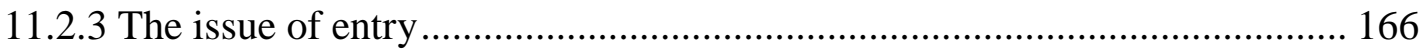

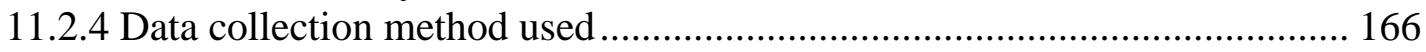

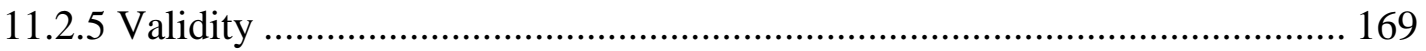

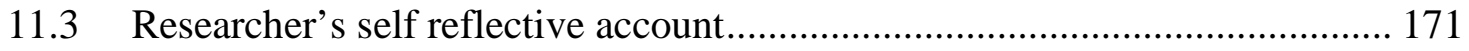

11.4 Chapters ahead ......................................................................................... 172

Chapter 12

The Activity Setting of Four churches......................................................................... 173

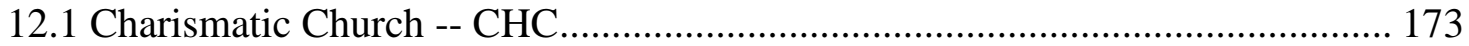

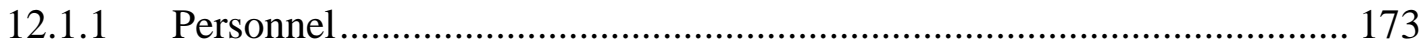

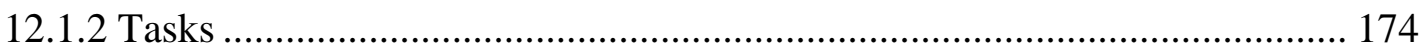

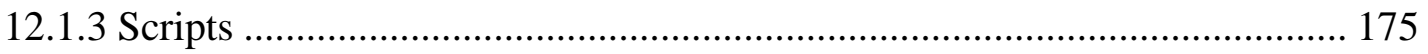

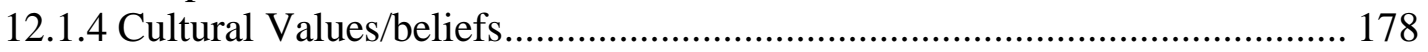

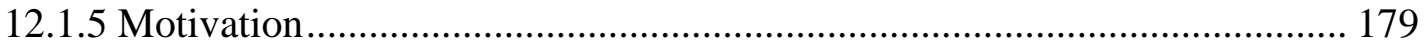

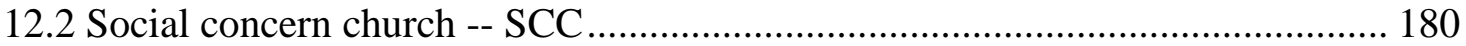

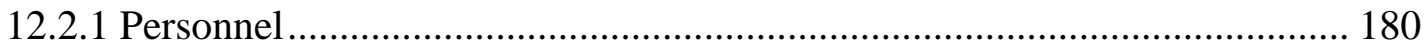

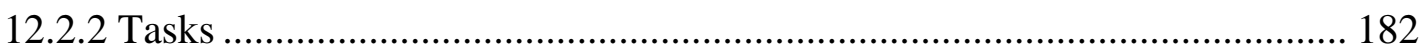

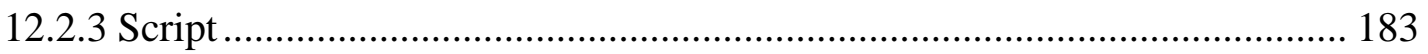

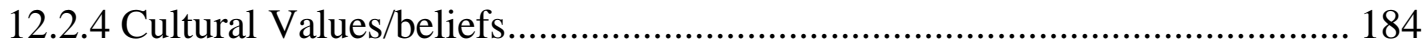

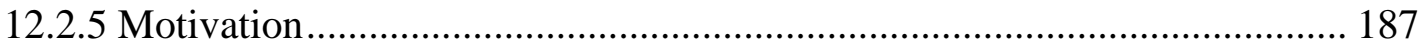

12.3 Fundamentalist Church -- FDC......................................................................... 188

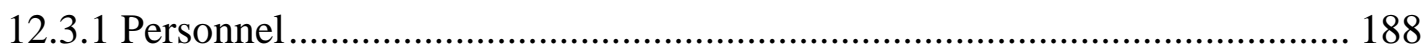

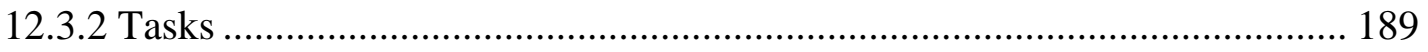

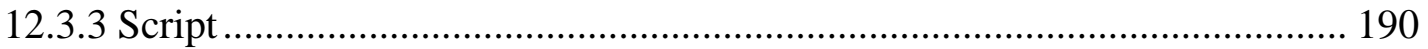

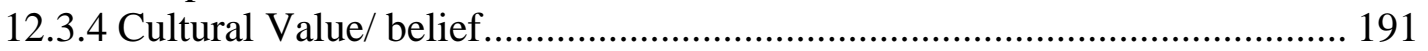

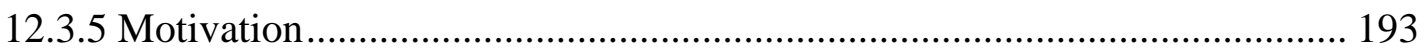

12.4 Evangelical Church -- EVC ................................................................................. 195

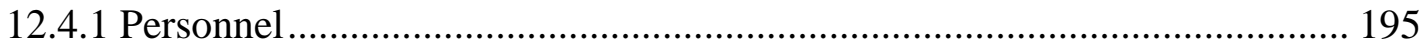

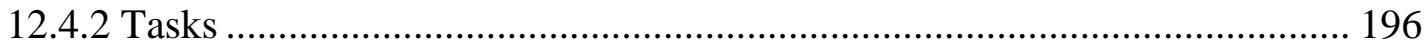

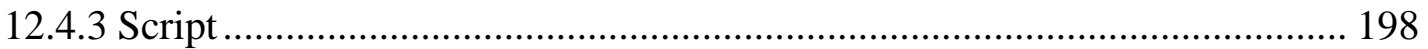

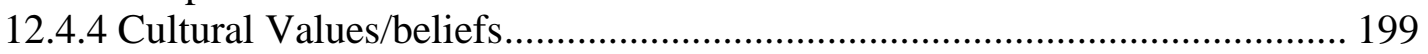

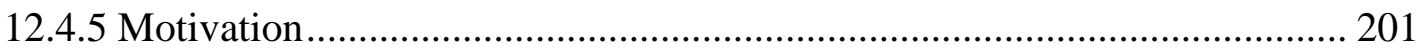

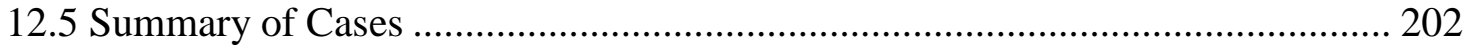

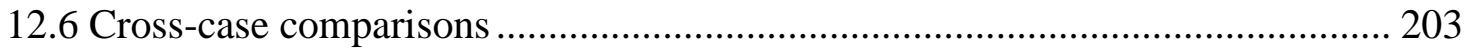

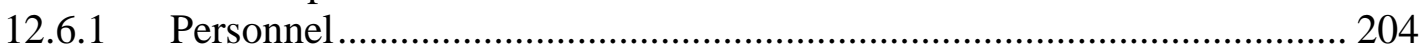

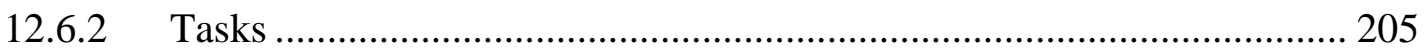

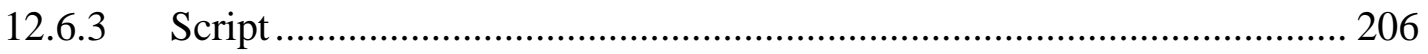

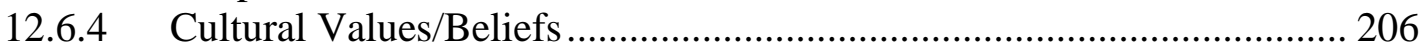

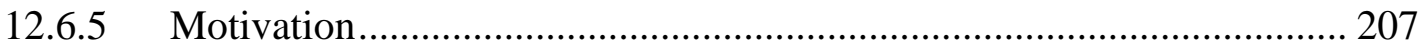




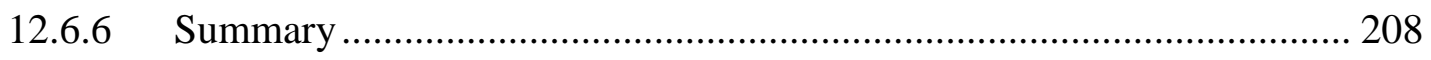

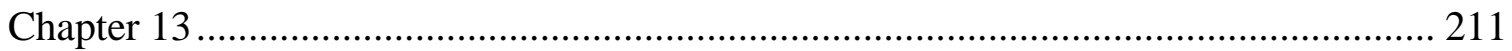

Findings and Discussion of the Qualitative Study ........................................................ 211

13.1 The effect of religion on well-being ………............................................... 211

13.1.1 Sense of community ....................................................................... 211

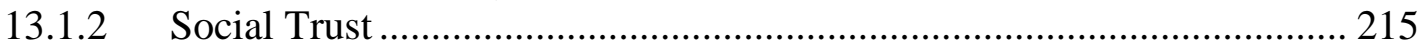

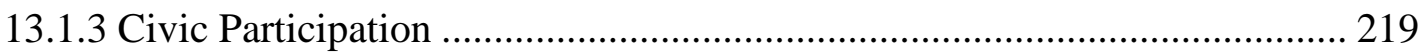

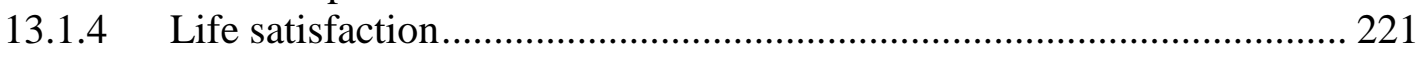

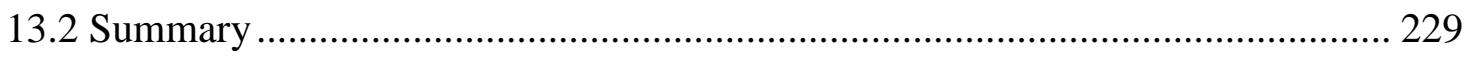

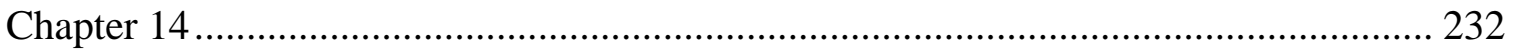

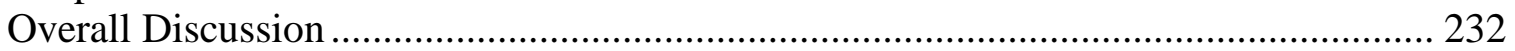

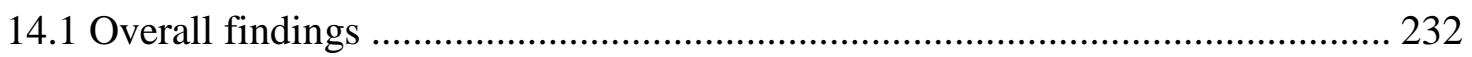

14.2 Contributions and Implications of the study ………......................................... 236

14.2.1 The importance of religion on people's well-being ...................................... 236

14.2.2 The importance of social context on people's well-being ............................. 239

14.2.3. The importance of mixed methodology ………………………………....... 244

14.3 Strengths and limitations of the study............................................................ 247

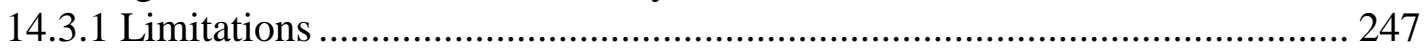

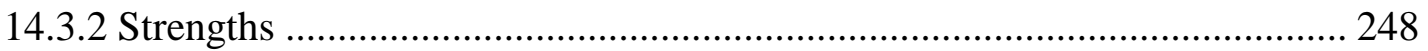

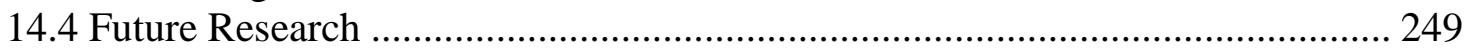

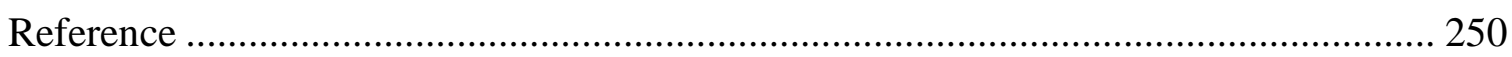

Appendix 1: Spirituality and Well-being Survey............................................................. 272

Appendix 2 _Assessing Model Invariance between the five settings ............................... 281

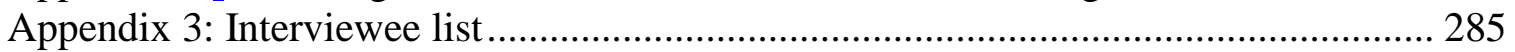

Appendix 4: Information Letter for Participants ....................................................... 286

Appendix 5: Consent Form for Participants ………………........................................ 288

Appendix 6 : Interview Guide.............................................................................. 289

Appendix 7: Social concern church activities................................................................ 291 


\section{List of Tables}

Table 1. Questionnaire responses …………………….......................................... 89

Table 2. Comparison of present study to the Hong Kong population data..................... 90

Table 3: Demographic data of studied settings ……………....................................... 91

Table 4: Cronbach's alphas for the scale .................................................................... 97

Table 5: Mean and standard deviations of variables among males and females and the

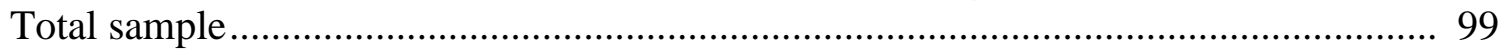

Table 6: Chi square test of settings by educational level .............................................. 100

Table 7: Chi square test of settings by monthly income ………….................................. 100

Table 8: Chi square test of settings by prayer and meditation.......................................... 101

Table 9: Chi square test of settings by bible reading .................................................. 101

Table 10: Chi square test of settings by serving in the church ...................................... 101

Table 11: Chi square test of settings by church attendance ............................................. 102

Table 12: Chi square test of settings by prayer and meditation...................................... 102

Table 13: Chi square test of settings by bible reading .................................................... 102

Table 14: Chi square test of settings by serving in the church ........................................ 103

Table 15: Chi square test of settings by church attendance .............................................. 103

Table 16: Chi square test of the setting by participation in voluntary organization....... 103

Table 17: Type of voluntary organization affiliation across setting ............................... 104

Table 18: Chi square test of settings by church activities participation ........................... 104

Table 19: Type of church activities participated by 4 church members ........................... 105

Table 20: Participants' personal identity across settings .................................................. 106

Table 21: Congregational identity perceived by members across settings ...................... 107

Table 22: Means and standard deviations of well-being variables by members across

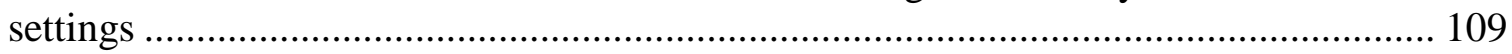

Table 23: Tests of the settings, sex and their interaction................................................ 112

Table 24: Tests of age (covariate), the settings, sex and their interaction ....................... 114

Table 25: Test of age (covariate) and the settings ........................................................ 117

Table 26: Means and standard deviations of spirituality variables by members across

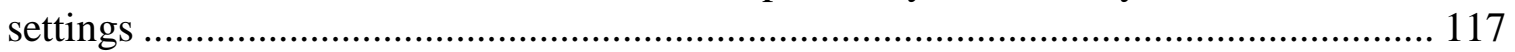

Table 27: Test of Age (covariate) and the settings ........................................................ 119

Table 28: Means and standard deviations of sense of community and social belief by members across settings............................................................................................. 119

Table 29: Wilks's lambda and canonical correlation for the five settings....................... 121

Table 30: Standardized discriminant function and structure coefficients for the five

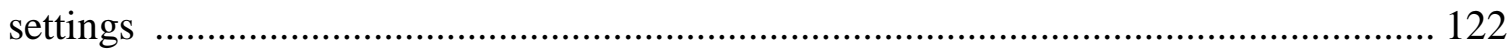

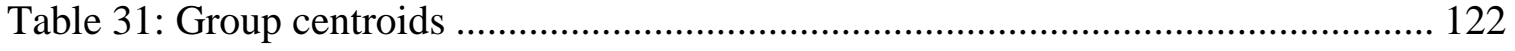

Table 32: Inter-correlations between all key variables ................................................... 125

Table 33: Stepwise Regression of spirituality related variables on life satisfaction....... 126

Table 34: Stepwise Regression of spirituality related variables on sense of community 126

Table 35: Stepwise regression of spirituality related variables on mental health ........... 127

Table 36: Stepwise regression of variables predicting life satisfaction ........................... 128

Table 37: Stepwise regression of spirituality related variables on social trust ............... 129

Table 38: Stepwise regression of variables predicting social trust .................................. 130 
Table 39: Stepwise regression of spirituality related variables on civic participation ... 131 Table 40: Stepwise regression of spirituality related variables on voluntary organization membership 132

Table 41: Stepwise regression of variables predicting civic participation. .................... 133 Table 42: Means, standard deviations, and correlations among the variables in path model 137

Table 43: Fit indices across endogenous variables, and chi-square difference tests for the models 138

Table 44: The activities participated by the researcher in each setting ........................ 167

Table 45: Synthesis of activity setting key dimension. 210 


\section{List of Figures}

Figure 1:Theoretical Model of the present study............................................ 54

Figure 2:Visual Model for Mixed Methods Design........................................... 88

Figure 3:Participants' personal identity across settings................................. 106

Figure 4: Congregational identity perceived by members across settings..................... 107

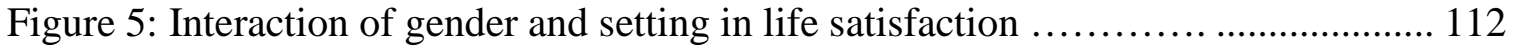

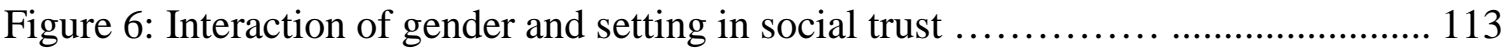

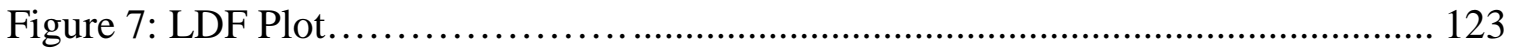

Figure 8: The hypothesized model of religion and well-being ................................... 135

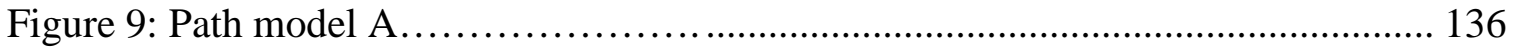

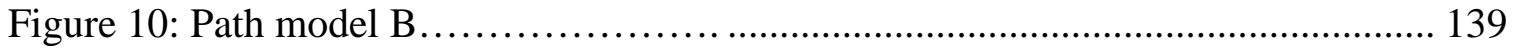

Figure 11: Path model C............................................................................. 139 


\section{Chapter 1}

\section{Introduction}

\subsection{Mental health and well-being}

What constitutes a good life (or well-being), what factors influence it, whether it can be promoted and how to attain it are all interesting and important questions appealing to a wide range of people. Philosophers or academics may debate whether well-being is concerned with people's happiness or human virtue, with subjective feelings or objective conditions, out of intellectual curiosity or in the interests of gaining insight into appropriate interventions. Policy makers may be more concerned about the operations of the concept and how it can be addressed by the use of public policy. The general public, however, may not be so concerned with how the term is defined, or whether or not the policy has explicitly addressed it: what they are interested in is how and what they can do to enhance their quality of life, or simply to feel good. Thus, it may be argued, mental health and well-being are everybody's business (2009a).

Although the diverse points of view just highlighted are only a small part of the public discourse on the issue, they are sufficient to provide us with an idea that wellbeing is a multi-faceted phenomenon. Is it hedonic or eudemonic in nature (Keyes, 2006; Ryan \& Deci, 2001)? Is it attributed to individual perception or objective conditions (Cummins, 1997; Diener, Sapyta, \& Suh, 1998)? Who can judge what well-being is? Can it be changed? If it can be changed, does any intervention have to come from individual or societal domains (Lyubomirsky, Sheldon, \& Schkade, 2005; Sirgy, et al., 2006)? Wellbeing is therefore not only an emerging research agenda, but also a critical issue for the individual as well as for societal development, because how the issue is viewed has a huge theoretical as well as practical, even policy, implication. This concern about wellbeing is especially apparent in a culture of surplus (Diener, 2009; Eckersley, 2004), which is concerned about living a life - not merely existing, but a quality existence.

In academic disciplines, although there are some (especially psychologists) who argue that well-being lies in the subjective perception of one's life or psychological functioning, others argue that well-being is closely related to, if not in, the objective conditions and broader environment of a person. Whichever approach taken, the concept 
of well-being has flourished to a large extent, either the concept itself or its measurement, through scholarly endeavour for several decades. For example, in psychology, mental health has long been associated with the absence of mental illness, rather than the positive notion of well-being. The major mission and task of psychologists was to treat problems, if not fix the person. Thus, not surprisingly, a considerable number of psychologists felt dissatisfied with the exclusive focus on the pathology of the discipline and later pioneered research exploring the positive nature of human psychology, such as subjective wellbeing, happiness, life satisfaction or other human character strengths and virtues (Diener, 1984; Diener, Suh, Lucas, \& Smith, 1999; Peterson \& Seligman, 2004; Seligman \& Csikszentmihalyi, 2000; Snyder \& Lopez, 2002). In the meantime, the social indicator movement or the recent notion of quality of life, more aligned with, and used at the policy level (because it takes seriously the objective determinants of health and wellbeing), is also acquiring more attention and momentum (Rapley, 2003; Sirgy, et al., 2006). Therefore, although the concept of well-being still needs further refinement, empirical research on well-being is shown to be not only feasible, but also promising. The next question is what can influence or even enhance people's well-being.

\section{$1.2 \quad$ Religion and Well-being}

There is compelling evidence that religion is a potent force in society, shaping both individuals and the larger society (Pargament \& Maton, 2000). The legacy of Pope John Paul II, the benevolence of Mother Teresa and the September 11 terrorist attacks are just a few examples of the influence of religion in the world. No matter whether its influence is good or bad, what we cannot deny is that religion is not only a personal and private matter, but can also play an important, if not decisive, role in the public sphere.

Nevertheless, religion remains strangely neglected by the social sciences, including psychology. The disagreement between psychology and religion is clearly shown by the disparaging stand on religion taken by influential figures in psychology such as Freud and Skinner. A lack of interest in, if not an active rejection of, religion is reflected in the psychological literature and in research. Fortunately, this situation has begun to change and interest in the effects of religion on human and social life has been renewed (Ebaugh, 2002; Emmons \& Paloutzian, 2003; Weaver, Pargament, Flannelly, \& Oppenheimer, 2006). In addition, as the world is urgently looking for ways and practices 
that can enhance people's well-being and quality of life, religion, as a traditional wisdom and human resource, and its beneficial and enhancing effects on personal and social wellbeing, has attracted increasing attention, and the results are also impressive (D. G. Myers, 2008; Paloutzian \& Kirkpatrick, 1995). In this way, in recent decades studies on the relationship between religion and subjective well-being, a host of human virtues, or even the building of community, have also been energetically pursued. No doubt the most recent WHO Melbourne charter, developed from the Fifth Conference on the Promotion of Mental Health and the Prevention of Mental and Behavioural Disorder, also regards spirituality as one important protective factor for mental health and well-being (VicHealth, 2009b).

Despite the impressive effect of religion on people's health and well-being, the religious community has rarely been explored, seeming to suggest that spirituality can be separated from the religion-based context. This is not, however, the stance of psychologists of religion (Pargament, 1999; Wulff, 1997), who regard spirituality and religion as two overlapping and closely tied concepts. Thus, following their perspective, the author of this thesis believes that, to understand the influence of religion on people's well-being, spirituality cannot be understood in isolation from the religious context. On the contrary, digging deep into the religious context in which people are embedded can uncover rich religious and socio-cultural resources people can draw on to enhance their well-being. In addition, religion, as a multi-faceted phenomenon covering religious belief, innermost experience and behaviour practices and also involving both private and public faces, is able to enhance people's well-being in a multiple and comprehensive way. Thus, when examining the relationship between religion and well-being, both concepts having many facets, a multi-dimensional rather than a simple linear understanding is important. All these aspects have become the foundation of the present study.

\subsection{Structure of the thesis}

The primary objective of the present study is to examine the impact of Protestant spirituality and religious community on people's well-being in the context of Hong Kong. Whether, how and why Protestant spirituality and religious communities can enhance people’s well-being are the key research questions to be explored. To this end, the thesis will be divided into three main parts: the background literature, socio-historical context 
and methodology of the study, and all the details of the mixed-method study, including both quantitative and qualitative study.

Chapters two to five present and clarify the key concepts and the basic principles of the study. Chapter two introduces the three major propositions about well-being in existing empirical research: well-being at subjective and individual levels, quality of life (QOL), and the multi-level understanding of well-being. While the first proposition is aligned with the most commonly used concept of well-being SWB (Diener, 1984), and the recently emerging positive psychology movement (Seligman \& Csikszentmihalyi, 2000), QOL (Cummins, 1997; Schalock, 2000; Sirgy, et al., 2006) goes beyond individual and subjective levels of well-being to concern about objective conditions. In contrast, the multi-level understanding of well-being (Cowen, 1991, 2000; Nelson \& Prilleltensky, 2005; Prilleltensky \& Prilleltensky, 2006) does not focus on specific levels, but suggests that well-being spans multiple levels and emphasizes the importance of the interrelationship between well-being at various levels. The major ideas of the three propositions are presented and also compared in relation to their underlying philosophical roots.

Chapter three explores the critical idea of a person in context, acknowledging the multi-level understanding of well-being. It is realized that the individual and the broader environment are interrelated, but how to understand the person in context is still far from clear. Perspectives and ideas drawn from sociological and psychological traditions, as well as some recently emerging perspectives, are presented and discussed. A convergence is observed within the different disciplines on the understanding of relations between person and environment: people are intertwined with their environment and cannot be understood in isolation from it.

Chapter four presents the existing (and impressive) empirical findings about the relationships between religion and well-being. It is shown that religions not only enhance people's well-being at the individual, interpersonal and collective levels, but also help build a community which sustains and maintains people's well-being, or even further strengthens the well-being of that community. However, the dark side of religion is also recognized and discussed in this section.

Chapter five summarizes the debate on whether religious phenomena can be studied together with the few major conceptualizations of religion: phenomenological, 
psychological, sociological and cultural perspectives. Integrating the insights of various perspectives, at the end of the chapter a multi-dimensional perspective of spirituality, drawing on the ideas of Dorr (1984) and Dokecki, Newbrough and O’Gorman (2001), is proposed as the guiding theoretical framework of the whole study. To put it simply, spirituality is an integral dimension of a person's religious belief, practice and relationship within a spiritually based context, and the manifestation of spirituality can span personal, interpersonal and social dimensions.

After providing an overview and the major concepts of the study, Chapters six and seven further situate the socio-historical context of the present study, and elaborate the methodology it employs. Chapter six provides a brief introduction to the four religious settings to be examined, and demonstrates how they are deeply embedded in the Protestant Christian environment as well as the socio-historical context of Hong Kong, so that a multi-layered understanding of the phenomenon can be revealed.

Chapter seven provides the rationale for using the mixed method approach in this study, and demonstrates the limitations of the conventional survey method and the contributions of qualitative research methodology. In short, the understanding of the religious context and its relationship to people's well-being is important in this study, but the survey method is not able to capture all the nuances in the settings, and a mixedmethod approach is thus better employed to shed light on the understanding of the phenomenon studied.

While Chapter eight to ten are related to the quantitative study, chapters eleven to thirteen are about the qualitative study. Chapter eight summarizes the details of the method used in the survey study, including participants, instruments and procedure. Chapter nine presents the major survey findings: descriptive findings, ANOVA/ MANOVA methods, discriminant function analysis and path modelling. In Chapter ten, the researcher discusses the findings in relation to the existing literature and also identifies gaps left unanswered by the survey study.

Chapter eleven introduces the activity setting framework as the analytical framework in understanding the fieldwork data and the use of multiple case comparisons to present the findings. In addition, the data collection method used in the field study and the validity issue are also specified. Chapter twelve presents the findings in the four churches, through the use of activity setting analysis. The five key dimensions - personnel, 
tasks, scripts, cultural values and motivation - are delineated clearly across the four religious settings, and the cross-case comparisons further help in comparing the similarities and differences between the various settings, so that insights illuminating the key research questions can be drawn on. In Chapter thirteen the researcher further uses the activity setting findings to help explain the significant survey findings shown in the earlier chapters, in combination with the existing literature. In Chapter fourteen the researcher integrates the findings and major insights drawn from both qualitative and quantitative studies to highlight the contribution of the whole study. Finally, the strengths and limitations of the study are highlighted, and directions for future research recommended. 


\section{Chapter 2}

\section{Well-being}

Health and well-being are common and generic concepts that concern governments because of their policy implications. Although the World Health Organization (WHO), since 1948, has adopted a broad concept of health as a "state of complete physical, mental and social well-being” (Larson, 1996, p. 181), health has long been just seen as free from disease. Similarly, mental health has often been equated with the absence of mental illness, while positive notions of mental health (e.g., well-being) have remained elusive with little empirical investigation (Keyes \& Waterman, 2003). Since the first publication on positive mental health (Jahoda, 1958), the conceptualizations and measures of well-being have progressed a lot in the past 50 years (Diener \& Suh, 2000b; Keyes \& Waterman, 2003), with the most commonly mentioned one being subjective well-being (SWB, Diener, 1984).

\subsection{Subjective Well-being}

\subsubsection{The components of subjective well-being}

There are two main streams of research in understanding subjective well-being: hedonic and eudaimonic (Keyes, Shmotkin, \& Ryff, 2002; Ryan \& Deci, 2001). The hedonic stream of subjective well-being is rooted in the Greek philosophy of Aristippus, who considered that life's ideal is to maximize pleasure and happiness. Its psychological construct, normally termed SWB, relates to an individual's feelings towards life and evaluations of affective states, including overall happiness and life satisfaction and positive and negative affective experiences. Life satisfaction, the cognitive perspective of SWB, relates to an individual's evaluation of his or her own life in general or specific terms. Happiness, a balance between positive and negative affects, reflects a person's immediate experience of pleasant or unpleasant moments. Thus, SWB considers the affective as well as the cognitive components of people's emotional experience towards life. 
In contrast, eudaimonic subjective well-being concerns the notion that true happiness is not just about following human desires, but also can find expression in "the expression of virtues - that is, in doing what is worth doing” (Ryan \& Deci, 2001, p. 145). Its psychological construct, psychological well-being (PWB), assesses a person’s psychological and social functioning as the criteria of well-being. Compared to SWB, which specifically focuses on subjectivity, PWB is a person's potential to realize a meaningful life and to meet real life challenges. The major proponent of PWB is Carol Ryff (Ryff, 1989; Ryff \& Keyes, 1995), who argued that SWB and PWB are related but distinct conceptions of well-being. For her, PWB involves a person functioning positively in six aspects of life: looking at oneself in appropriate way (self-acceptance); having good relations with others (positive relations with others); having a sense of growth (personal growth); realizing a meaningful life (purpose in life); having the capability to manage the surrounding world (environmental mastery); and a sense of self-determination (autonomy). Other theorists define fully functioning people are those who can actualize themselves, in the area of autonomy, competence and relatedness (Ryan \& Deci, 2001), who live according to the true self (Waterman, 1993), or who strives for a good balance between happiness and meaningfulness (McGregor \& Little, 1998).

Keyes (1998), following Ryff, argued that, in addition to psychological functioning, to attain well-being people have to function well in regard to social challenges and tasks. He suggests five dimensions of social well-being: social integration, social contribution, social coherence, social actualization and social acceptance. Social integration concerns the individual's capability of being part of society. Social contribution is the degree to which a person can offer something valuable to the world. Social coherence is about whether a person finds what is happening around them is understandable. Social actualization refers to whether a person can envision the future and potential of a society. Social acceptance is about whether a person can trust and accept both good and bad of human nature. In summary, Keyes argues that attaining wellbeing goes beyond private life, towards good functioning in the public domain. He suggests that people who achieve all three aspects (SWB, PWB and social well-being) can be described as having complete mental health: they are flourishing. Otherwise, they could be said to be languishing (Keyes \& Lopez, 2002). 


\subsubsection{Positive Psychology Movement}

In line with the growing study of subjective well-being, positive psychology is another major force within psychology that pursues the brighter side of human nature. Seligman and Csikszentmihalyi (2000) contend that psychology needs to shift its focus from correcting the weaknesses of individuals to exploring human strengths and virtues. The underlying goal not only is to prevent further serious mental problems, but also to build and develop flourishing and fully functioning people. These goals have been advanced in the last two decade by research into positive emotion (Fredrickson, 2000, 2001; Isen, 1987), optimism (Scheier \& Carver, 1985), learned optimism (Seligman, 1991), hope (Snyder, 1994, 2000), courage (Rate, Clarke, Lindsay, \& Sternberg, 2007; O' Byrne et al. 2000 cited in Snyder \& Lopez, 2007), wisdom (Sternberg, 1990, 1998), gratitude (Emmons \& McCullough, 2004), forgiveness (McCullough, 2000; McCullough, Pargament, \& Thoresen, 2000), mindfulness (Langer, 2002), flow (Csikszentmihalyi, 1990), and spirituality (Larson, 1996; Pargament, 1997, 2002a).

Peterson and Seligman (2004) developed the VIA Classification of Strengths as the antithesis of the DSM category system. Although the universality of these virtues and strengths is still under debate, the fruits in this area of research are abundant and the advancement in empirical research into human strengths is impressive. Nevertheless, it should be noted that, like PWB, study into different kinds of human strength is still in the early stages. Most research into well-being focuses on SWB (of the hedonic tradition), while the eudaimonic approach only received attention in the 1980s onwards (Keyes, et al., 2002).

The differences between SWB and PWB or other human strengths, indicates the divergence of hedonic and eudaimonic approaches to well-being, and their differing underlying philosophical roots (Ryan \& Deci, 2001), but it also brings to light a more indepth discussion about whether well-being should be defined by individuals or others (like expert or normative values), and whether well-being can be universally or culturally applied. Although SWB is criticised as self indulgent, Diener and his colleagues reiterated that SWB pays more respect to people's own values and judgments, avoiding hegemony from external judgment (Diener, et al., 1998; Diener \& Suh, 1997). In addition, Diener et al. (1998) remarked that PWB, presumably universal, may vary across culture. They suggest that, while PWB may contribute to the understanding of well-being, SWB 
is still essential to well-being. In view of the widespread use of SWB and the emerging development of PWB as indicators in well-being research, more understanding and conceptualization on subjective well-being is anticipated. It also seems that complementing SWB with PWB may provide a better picture of the complex construction of well-being (Keyes, et al., 2002; Ryan \& Deci, 2001).

Despite different conceptualizations, the commonality and major focus of the aforementioned perspective of well-being has been on the personal and subjective realm.

\subsubsection{Correlates of subjective well-being}

As well as defining well-being, research also strives to understand the correlates of well-being, including antecedents and consequences. As discussed, we draw heavily on the study of SWB. Wilson's (1967) classic Correlates of Avowed Happiness tells us that happy people are those who are "young, healthy, well educated, well paid, extroverted, optimistic, worry free, religious, married person with high self esteem, high job morale, modest aspiration, of either sex and of a wide range of intelligence” (p. 294). More than three decades have passed since Wilson's classic, and studies quite consistently show the close relationship between people's SWB and the following contributing factors (Diener, 1984; Diener, et al., 1999):

\subsubsection{Demographics (Age, Income \& Education)}

Even more than wealthy people, those with higher education, and the young, were shown to be happier (Wilson, 1967), but later studies have shown that this is not the whole picture. Although wealthier people are generally happier than the poor, the impact of wealth on SWB is small and limited, especially in rich countries (Diener, et al., 1999). In contrast, wealth may have negative impact on people's SWB because wealth may bring more stress, or people may pursue materialistic gain at the expense of their inherent psychological needs (Kasser, 2000). In the same way, there is a significant, but small, relationship between education and SWB (Diener, et al., 1999). However, because of the high correlation between income, education and occupational status, researchers have found it difficult to distinguish their independent effects. They also explain that, education, like income, is a means for people to achieve their goals, rather than the ends. In certain cases, higher education may lead to distress because it raises expectations that cannot be met (Diener, et al., 1999). In terms of age, even though older people’s strong 
positive emotions may decline with age, their satisfaction towards life does not decline, and perhaps may increase (Diener, et al., 1999). This not only reflects the fact that the elderly can adapt quite well to old age, but also indicates that the elderly are now enjoying more opportunities and social advantages than in the previous generations.

\subsubsection{Physical states (Health, Exercise)}

The relationship between health and SWB is, despite expectations, not definite. Research shows that SWB is only related to perceived health, rather than objective health (Diener, et al., 1999). Researchers have argued that people adapt to worsening health by appraising their health positively, using downward comparison strategies or downplaying "the importance of their health when evaluating their global life satisfaction" (Diener, et al., 1999, p. 287). Nevertheless, Diener admits that when the disability is severe, the adaptation cannot mitigate the negative impact of ill-health on SWB.

In addition, the beneficial effects of doing exercise on greater happiness have been documented (Argyle, 2001; Carr, 2004; Penedo \& Dahn, 2005). The short term effects are related to the release of endorphins which have an enhancing effect on the brain, while long term effects related to exercise are better health and stronger social support, resulting from having company in the activities. A recent study (Joiner, Hollar, \& Van Orden, 2006) even found that fewer suicides occurred on the Super Bowl Sundays compared to non-Super Bowl Sundays, supporting the sport-related “pulling together” effect.

\subsubsection{Relationships (Marriage, friendship, religion)}

The positive and beneficial effects of marriage are enduring, across the time and culture (Argyle, 2001; Diener, et al., 1999; Myers, 1999; Myers \& Diener, 1995). Marriage not only provides people emotion and economic support, but also provides structured opportunities of interactions and role sharing, together contributing to life satisfaction. Nevertheless, the negative impact of divorce or stressful marital relationships is also clear, especially in individualistic countries. Williams’ (1988) study showed that it is the quality of marital relationships, rather than the marital status, that is important for individual well-being. Shapiro and Keyes’ (2008) study even demonstrated that the advantage of married persons over non-married persons, related to psychological 
well-being, does not exist in terms of social well-being. In addition, it is worth noting that recent research findings show that the benefit of marriage for males is higher than for females (Nock, 1998). That may be because, as Nock explains, marriage changes men in several aspects: achievement, participation in social life, and philanthropy, by reinforcing such kinds of behaviour in more integrated male human development. Williams’ (1988) study also supports the differentiated effect of marriage (related to gender) as he finds that "the effects of marital quality are stronger among women than among men” (p. 452).

Other social relationships, like friendship, also are also related to, and contribute to, people's mental and physical health. In the recent decades, the positive impact of spirituality and religion on peoples' well-being has become the focus of many researchers, because of advancement in the methodology and measurement of religious variables. Detailed discussion will be presented in Chapter 5, but the general finding is rather positive (D. G. Myers \& Diener, 1995), even though the negative effect is acknowledged.

\subsubsection{Environment (Wealth, culture)}

In addition to individual differences in SWB, national differences are also another focus of researchers who strive to understand the mechanisms and cultural roots of happiness of people from different cultural backgrounds (Diener \& Suh, 1999, 2000a). Studies indicate that people living in the countries with more wealth, an individualistic culture, income equality, a stronger emphasis on democracy and human rights, are happier and more satisfied with their life, despite the variables being highly correlated (Argyle, 2001; Diener \& Suh, 1999). It should be noted that health disparities grow with wealth disparities (Wilkinson, 1996) and social inequality can reduce SWB (Veenhoven, 2008). Furthermore, people living in countries with higher interpersonal trust were also found to have higher SWB. Therefore, the broader environment may influence people's being by affecting people’s social relations.

Studies into the correlates of PWB have been conducted by Ryff and Keyes (Keyes, 1998; Keyes, et al., 2002; Ryff \& Keyes, 1995). Demographic factors (including age and education), and personality factors were shown to be related to PWB. Correlates of various human strengths are also summarized in Snyder and Lopez (2002, 2007). They argue that while human strengths have a neurobiological base, the influence of childhood 
antecedents, the social environment, and the cultural context, are also critical to the development of people’s behaviours, including their well-being.

\subsubsection{Mechanisms of subjective well-being}

Apart from just describing the characteristics of happy people or happy countries, recent studies into well-being also dig deeper into the underlying processes or principles that make people or countries happier. The main reason is that the external and objective variables can only account for a small part of the variance in SWB (Diener, et al., 1999). The following are important variables relevant to the present study.

\subsubsection{Goals}

Diener et al.(1999) recognized the benefits of attaining goals in emotional and life satisfaction. In general, people react in positive ways when making progress toward goals and react negatively when they fail to achieve goals. This can help explain why richer people, with more resources, are normally happier, because they can more easily attain their goals. Conversely, it can also explain why prisoners, who have intimate relationships with people outside the prison, have lower well-being. Nevertheless, Diener et al. (1999) also realize that whether attaining goals has beneficial effects largely depends on whether the goal is matched with basic motives and needs, and whether the goal is aligned with cultural norms and expectations. In short, those pursuing extrinsic

goals (such as wealth and fame), rather than human needs (such as close relationships and self acceptance), were found to have lower well-being (Kasser, 2000; Kasser \& Ryan, 1993). Those pursuing goals contradictory to cultural values may also be less satisfied with their life (Kitayama \& Park, 2007; Oishi, 2000). Therefore, the study of goals in relation to SWB provides us with a deeper level of understanding between SWB and its correlates.

\subsubsection{Adaptation and Coping}

The fact that lottery winners are not necessarily happier than ordinary people, and that individuals with spinal cord injuries are not necessarily especially unhappy (Brickman, Coates, \& Janoff-Bulman, 1976 cited in Diener, et al., 1999), led researchers 
to suggest that adaptation plays a significant role in moderating people’s SWB.

Nevertheless, (Diener, et al., 1999) also remark that there is also a large amount of evidence against the "hedonic treadmill" theory - that is, that people adapt to all kinds of situations, such as carers who care for patients with chronic illness, or people facing extreme poverty. Diener et al. point out that adapting well to situations is closely related to coping strategy, goals, and structures.

\subsubsection{Cultural norms}

Besides these individual variables, the individual and national differences related to SWB may also be attributable to the cultural norms (Diener \& Suh, 2000a). The effect of culture on SWB is not direct, but mediated by the self (Suh, 2000), goals (Oishi, 2000), and interpersonal relationships (Kitayama \& Markus, 2000), through influencing motivation, cognitive factors or interpersonal interaction patterns.

Understanding these variables can help us make sense of differences in individual well-being under any given situation. It should be highlighted that most of these variables are closely related to environmental influences. Therefore we can understand that wellbeing is not just an intrapsychic matter, but is closely related to context. In summary, while the correlate study of SWB provides a general picture of what kinds of people are happier, the study of the mechanism of SWB provides a deeper understanding of how life situations affect people’s SWB.

\subsection{Quality of life}

\subsubsection{The main concept}

Another approach towards understanding good life or well-being is research into quality of life (QOL). QOL is a complex and still debatable construct that has been employed by different disciplines to delineate what a good life is. Further debate exists relating to the individual or collective level (Carr, 2004; Rapley, 2003; Sirgy, et al., 2006), concerning populations and individuals. It is debatable because there is no consensus regarding whether QOL should be objectively or subjectively measured, whether it should be defined by researchers or participants, or whether the concept of QOL can be applied universally or should be culturally defined. 
QOL has long been associated with economic indicators (such as GDP or income levels). The social indicator movement, popular in the 1960s, aimed to provide a broader measure of quality of life within a nation, not just monetary indicators; in recent decades, there has been QOL research into individual and subjective perspective of life (Rapley, 2003). Some even say that QOL is primarily subjective (Haas, 1999). Nevertheless, whether at the individual or population level, it is broadly agreed that QOL is a multidimensional construct, embracing both objective and subjective domains, despite the fact there is no consensus over the number of dimensions or definition of QOL (Costanza, et al., 2007; Cummins, 1997; Diener \& Suh, 1997; Rapley, 2003; Schalock \& Felce, 2004). Generally speaking, the definition used in research largely depends on the purpose of research and stance of the researcher.

With a goal to inform intervention and social policy, some national well-being indicators focus on comprehensive ranges of factors that may affect the quality of life related to individuals and society as well (Rapley, 2003). They include indicators related to housing, crime and safety, work, education, health, as well as family and the community. Drawing on 11 basic human needs, such as security, affection, leisure, reproduction and participation, Costanza et al. (2007) constructed a QOL framework in relation to various kinds of capital which can be advanced by policy implementation. So, we can say the content of QOL, as opposed to SWB, is more compatible with policy making, as it deals with the objective environment.

In psychology, Cummins (1997) has proposed the following definition: "Quality of life is both objective and subjective, each axis being the aggregate of seven domains: material well-being, health, productivity, intimacy, safety, community, and emotional well-being. Objective domains comprise culturally-relevant measures of objective wellbeing. Subjective domains comprise domain satisfaction weighted by their importance to the individual” (Cummins, 1997, p. 6).

Similar to Cummins, Schalock (2000) proposed a similar QOL conceptualization that is specifically relevant, but not exclusive, to people with intellectual disabilities. Drawing on a wide range of literature, Schalock proposed eight dimensions (emotional well-being, interpersonal relations, material well-being, personal development, physical well-being, self-determination, social inclusion, rights) as the core of quality of life. In 
each dimension, subjective personal appraisal and objective functional assessment are measured independently.

While Cummins (1997) covers more culturally relevant domains (e.g., safety and community), Schalock (2000) emphasizes autonomy and individual rights, particularly related to people with intellectual disabilities. They both, however, consider the importance of objective and subjective measurement of life, at the individual level. Schalock and Felce (2004) even suggest that that we should use the objective indicators of life experiences, instead of SWB, to evaluate the environmental impact on people in a more sensitive way.

Compared to the social indicator tradition that emphasizes objective external circumstances, Cummins and Schalock both focus on people's inner perceptions and satisfaction. In contrast to the SWB or PWB only considering people's happiness, feeling, and human functioning, Cummins' and Schalock's definitions of QOL concern both the subjective and objective evaluation of ones' situation, and in multiple domains that are culturally important and normatively relevant. Irrespective of whether those different domains are universal, Cummins’ and Schalock’s QOL definitions help bridge the traditional objective measures of the social situation of the population and the subjective evaluation of the individual. Their conceptualizations imply that objective indicators of the population affect the objective domains of individuals, which in turn affect the SWB of people.

As Michalos (in Sirgy, et al., 2006) argues, construction of QOL models is a political and philosophical exercise rooted in specific kinds of values. Whatever the model or conceptualization of QOL we adopt, it can be debated. Theoretically, there are rich accounts of approaches to quality of life involving different underlying philosophies: utility, needs, prudential values and capabilities, as summarized by Phillips (2006). According to Phillips, those that embrace utilitarianism envision and strive for societies which enable their citizens to achieve the greatest happiness. In contrast, those who adopt prudential values emphasize lives based not on people’s desires, but on values which make humanity better. Those values include values similar to the utilitarian approach -enjoyment and avoidance of pain -- but also include accomplishment, basic capabilities, understanding, liberty, self respect, deep personal relations, which are believed to enhance life in a general intelligible way. Those emphasizing needs contend that every 
human being should be entitled to a minimum level of basic needs, such as food, water, shelter, education. While this concept is simple, it provides no idea as to what quality of life should be. The capabilities approach is a kind of mix of the previous three approaches and regards quality of life as being about a person's “ability to do valuable acts or to reach valuable state of well-being” (Phillips, 2006, p. 91). This understanding of QOL takes both the external environment and human desire and need into consideration. Nevertheless, what constitutes an elementary capability set can vary in the literature.

\subsubsection{Quality of Life and Subjective Well-being}

In discussing Cummins’ and Schalock’s understandings of QOL, some comparisons have been made between the concepts of Quality of Life and Subjective Well-being. However, deeper reflection between the two concepts should be explored, especially related to underlying philosophy.

Subjective well-being emphasizes the personal level, including life satisfaction, happiness and human functioning; whereas quality of life is a broader concept, including life in multiple dimensions and embracing both subjective and objective domains, at the level of the individual as well as the population. Simply speaking, subjective well-being can be seen as a subset of quality of life, not accounting for external circumstances. However, because PWB is not being fully conceptualized and SWB indicators are in widespread use in subjective well-being research, subjective well-being is dominated by a narrow focus on the feeling and hedonistic approach to well-being, ignoring other psychosocial dimensions of well-being at the personal level. Despite this, Phillips (2006) still argues that QOL should start with subjective well-being, especially SWB, because it takes individual's judgment seriously. Otherwise the human autonomy would not receive enough respect.

Quality of life, however, does not stop there. Many studies indicate that subjective and objective well-being are not necessarily correlated (e.g., Phillips, 2006; Schalock \& Felce, 2004). So, a sole focus on subjective dimension without attending to social dimensions is inadequate, if not naïve. Some even use the term "happy slave” or "Fool's Paradise" to describe the contradictions of those who adapt quite well and report high levels of SWB, but suffer high levels of deprivation (Phillips, 2006; Sirgy, et al., 2006). As a result, many researchers have proposed that the best way of measuring QOL is to 
combine objective and subjective indicators because they each provide valuable information that can enhance quality of life and inform policy intervention (Costanza, et al., 2007; Diener \& Suh, 1997; Schalock \& Felce, 2004).

In relation to Phillip’s (2006) four main approaches to QOL, SWB is closely aligned to the utilitarian tradition and individualistic perspective, as it maximizes pleasure and avoids pain (Diener \& Suh, 1997; Phillips, 2006). PWB, or other human strengths, relate more to the prudential values and capabilities approach in which realizing potential and striving to make human life better is the goal. The prudential values and capabilities approach takes the objective environment more seriously and is more collectivist. Although the basic needs approach is one way to consider QOL, minimum standards related to human existence provides little vision of human nature (Phillips, 2006).

\subsubsection{Health Related Quality of Life (HRQOL)}

There is a long association between health and good life, in both the philosophical and medical fields. That is also why the WHO has long defined health using a holistic perspective, defining it as a "state of complete physical, mental and social well-being” (Larson, 1996, p. 141). As a result, health, well-being, QOL and health related QOL are “often used interchangeably in the health field” (Sirgy, et al., 2006, p. 401), manifested in Bowling's (1997) and Fitzpatrick’s (1996) definitions of health related to quality of life, incorporating wide ranges of human functioning, including physical health, emotional state, or social roles. These generic measures of health and QOL are generally used in national health surveys to monitor a nation's health (Rapley, 2003; Sirgy, et al., 2006). Despite not being as broad a concept as QOL, HRQOL conceptualization and measurement, especially the wide-ranging and multidimensional types, can be understood as quality of life in general (Michalos, 2004; Phillips, 2006). Indeed, Michalos suggests that, for clarity, we abandon the term "health related quality of life".

The wide ranging and amorphous nature of HRQOL is little use to health professionals, particularly if they need recommendations to inform decisions about life and death or treating illness, or to evaluate different medical procedures or policies. Therefore, a more impersonal, scientific and objective approach to HRQOL has been proposed in the field. Some of examples are Quality Adjusted Life Years (QALY) or Disability Adjusted Life Years (DALY), and time-trade off method (Phillips, 2006; 
Rapley, 2003). These approaches are useful because they provide a straightforward statistical method that can inform health practitioners in making medical decisions after comparing the relative costs and effects of different procedures, and the risks involved. They are, however, debatable because the patients' point of view is not highly regarded, and the ethical issues are centred around the relationship between value of life and quality of life (Phillips, 2006; Rapley, 2003).

The dilemmas of using QALY bring out a critical issue that physical health does not necessarily equate to having health related quality of life, let alone overall quality of life (Michalos, 2004). Apart from health measured in both objective and subjective senses, there is still more to contribute to overall quality of life, which we will examine in more detail in the following section.

\subsubsection{Community and Ecological level of QOL}

The social indicator tradition brings social circumstances to close attention, beyond monetary concerns; however, there is a lack of focus on the community context in which QOL is embedded. Raphael et al. (2001) recognize how community structure affects individual well-being. They warn that neo-liberalism threatens and deteriorates community infrastructure that is crucial to the building of strength and support between and within communities. The recent emergence of the community indicator movement also highlights the importance of satisfaction at the community level to overall well-being (Dluhy \& Swartz, 2006; Wiseman, 2009; C. Wong, 2002). Rapley (2003) argues that large scale indices (e.g., GDP, a fair and low crime society, a vigorous cultural life) are too distant from the concerns of local communities and citizens. Therefore, work on community indicators can bridge the gap and provide valuable information to policy makers at the local level, to link community development to the locally meaningful indices of QOL. While some proposed community QOL indicators may be members of the community's satisfaction with government, business, and non-profit services (Sirgy \& Rahtz, 2006), there are numerous other indicators adopted by different communities addressing various needs across various regions (Dluhy \& Swartz, 2006; Sawicki, 2002; Wiseman, 2009). Nowadays, more and more scholars suggest that community indicators should be developed through community consultation, with intellectual input from academia (Dluhy \& Swartz, 2006; Rapley, 2003; Sawicki, 2002; C. Wong, 2002). In 
addition to the individual and national levels, the community indicator approach suggests that there is an intermediate layer to focus on, because the community can play a significant role in developing well-being.

In summary, research into QOL presents a tension between a state of states versus as state of persons. It also indicates the necessity of an interdisciplinary approach to investigating such a complex issue. Nevertheless, Rapley (2003) argues that large scale indices (at the national level) may mask real differences between population subgroups and such indices are from the concerns of individuals or local communities. Community social indicators have been emerging in the recent decade. We can now examine wellbeing from a holistic and comprehensive perspective at individual, relational and community levels.

\subsection{Multi-Level approach to well-being}

\subsubsection{The major proposition}

Instead of targeting well-being at a specific levels (such as self esteem or community safety), some have proposed that well-being should be understood in multiple levels simultaneously. Cowen’s (1991, 2000) concept of “wellness” and Prilleltensky et al (Nelson \& Prilleltensky, 2005; Prilleltensky \& Prilleltensky, 2006) define well-being as beyond the personal and intra-psychic, requiring divergent solutions across different levels. Cowen $(1991,2000)$ argue that wellness is not “on/off”, but is a continuum. Furthermore, wellness is also not once-and-forever; it can be nurtured or inhibited by external circumstance. In addition, wellness depends on many aspects of life; there is no simplistic solution, but only comprehensive strategies targeting various levels. Cowen specifically highlights several sources of influence that have tremendous impact on wellness: (a) families in which children naturally grow up; (b) educational experience; (c) significant social setting or mediating structure in which people interact with each other; and (d) larger society. To promote wellness, Cowen proposes several concepts (competence, resilience, social setting modification and empowerment) that can be explored in more depth, so that greater understanding and subsequent intervention in specific areas can be obtained.

Prilleltensky and colleagues further argued that well-being is deeply embedded in the social environment including in family, community and society and that different 
aspects of well-being are closely intertwined (Nelson \& Prilleltensky, 2005; Prilleltensky \& Prilleltensky, 2006). "Well-being is not either personal, organizational, or collective, but the integration of them all. For any one of these spheres - personal, organizational, or collective - to experience well-being, the other two need to be in equally good shape” (Prilleltensky \& Prilleltensky, 2006, p. 11). To understand well-being in holistically, Nelson and Prilleltensky (2005) emphasise the importance of the personal (such as self esteem, independence), the interpersonal (having a supportive relationship and freely engaging in society), and collective levels of well-being (ability to acquire community resources).

As Prilleltensky and Prilleltensky (2006) explain, without the contribution of optimistic people, who are effective and have a sense of purpose, organizations cannot function properly. Similarly, people cannot flourish without an immediate environment that is stimulating, respecting, affirmative and reflective, as well as a broader society that is resourceful, fair and free of corruption. In other words, to enable people to be optimistic and effective, to be determined and to grow personally, an enabling organizational structure and a just community is necessary.

\subsubsection{Comparison between different conceptualizations of well-being}

The multi-level understanding of well-being can be compared with subjective well-being and QOL in several aspects. Firstly, Prilleltensky and Prilleltensky’s (2006) multi-level model of well-being is explicitly collective in nature, so individual enjoyment is not a primary concern. Although individual self determination, freedom and personal growth can be highlighted, it should be balanced with values at other levels, such as collaboration, respect for diversity or social justice. Otherwise, personal well-being will be confined. Therefore, we can see that Prilleltensky's model is distinct from the SWB or utilitarian tradition, and more in line with PWB in subjective well-being, and the prudential value approach to quality of life.

Secondly, the multi-level understanding of well-being complements the traditional disciplines which focus on either the personal or macro level of analysis. Instead, it realizes the critical role of the intermediate structure, which can play an enhancing or inhibiting role in bridging the individual and society. Thirdly, and most importantly, the multi-level approach to well-being acknowledges the importance of the interrelationship 
of different levels, instead of targeting a certain level. This is in line with social epidemiology’s claim that social relations matter in health. Last, it provides a comprehensive framework that we can build on to explore diverse disciples in relation to well-being.

This thesis will explain why a multi-level understating of well-being is so important and delineate how and why the interrelation of different levels (individual and social) is able to influence well-being. After that, this thesis will outline the model of well-being used in the present thesis.

\subsubsection{Why multi-level understanding of well-being is important}

In psychology, well-being has long been studied at the individual level. It was shown, however, in the previous section (2.1.4), that it is more fruitful to understand well-being by examining a few important variables (such as goal, adaptation or personality) and to explore the underlying mechanism. Nevertheless, individual goals and coping strategies, or even personality, do not arise in a vacuum, but are closely related to the social or cultural environment. Therefore, understanding well-being without attending to the social environment is limiting.

Furthermore, O’Neil (2005) cogently argues that the way we look at problems or issues affects how we study them and try to solve them, and this has ethical implications. Regarding the concept of well-being, if well-being is to be conceptualized only within certain level, strategies targeting that specific level will result, without attending to intertwining relationship between people and external circumstance. As previously argued, if well-being is to be conceptualized just within the individual, with no regard to the external circumstance, a "happy slave” or “fool’s paradise” may result. Strategies targeting individual or adjusting individual perceptions and cognitions may also arise. At worst, no hope exists if well-being is heredity or genetically determined. Similarly, if well-being is totally beyond individual perception, as is proposed by the social indicators approach, nothing can be done, except political advocacy. People will be falsely perceived as passive recipients of the environmental influence. However, a multi-level understanding of well-being requires a system perspective that is transactional, and reciprocal influence between the multiple levels should be explored to attain a clear understanding of well-being. In addition, subsequent intervention would also require 
multiple solutions, rather than a single solution, to fully address the well-being at multiple levels.

The multi-level understanding of well-being highlights the mediating role of the immediate setting or organization or smaller scale of community between person and society. This matches with QOL research focusing on organization (Sirgy, et al., 2006) and community social indicators (Dluhy \& Swartz, 2006; Sawicki, 2002; Wiseman, 2009). In view of citizens losing trust in government and bureaucracy, Berger and Neuhaus (1977) suggest that a mediating structure is needed between individuals and larger institutions, otherwise social policy will become detached from the realities of individual life. In other words, immediate settings can help to mediate between the micro and macro aspects of life; organizations can have impacts on the individual, whether good or bad. Therefore, understanding how this mediating structure interacts with wellbeing deepens our understanding of well-being, and also provides us with ideas for interventions to enhance well-being.

Lastly, a multi-level understanding of well-being emphasises the importance of interrelationships (between people and the environment) in well-being. This is in line with recent advancements in epidemiological studies. For example, the famous Whitehall studies of British civil servants (Marmot, Shipley, \& Rose, 1984; Marmot \& Wilkinson, 1999) indicated the impact of social hierarchy on ill health. Berkman and Glass (2000) illustrate how social networks influence health by providing social support, mutual influence, opportunities for engagement, close personal contact and resources, and material goods. Diez-Roux called for context in epidemiology (1998), and suggests using multilevel analysis to explore the complexity and reciprocal relationships between factors operating at different levels (2000).

A wide range of studies indicate the importance of social relations to health and well-being. Helliwell and his colleagues (2002; Helliwell \& Putnam, 2004) also substantiate that community well-being (manifested in higher social capital) is strongly linked to SWB. Baumeister and Leary (1995) and Myers (1999) ascertained that humans have a fundamental need to belong and that good interpersonal relationships are conducive to health and well-being. Therefore, all these findings indicate that different facets of well-being are closely related. The author of the present thesis also believes that individual well-being is not only about health and psychological state. Well-being is 
manifests across many levels and is closely associated with the individual's relations to the self, others, and society.

\subsection{A model of well-being}

In the present thesis, well-being is conceptualized as a multiple level construct.

Although the well-being at the community and cultural levels is not examined in detail because of time constraints, well-being at the individual and organizational levels is explored. Regarding well-being at the individual level, both SWB (manifested in measures of life satisfaction) and PWB (manifested in social trust and participation) will be used so that the strength of both traditions can be drawn upon. Moreover, health will also be measured because of its close relation to well-being. Regarding well-being at the organizational level, the goal, size and sense of community within the organization will be focused on in relation to impacts on individual well-being. The framework is comprehensive, and examining the interplay between the individual and the environment is not an easy endeavour, as will be discussed in the next chapter. 


\section{Chapter 3}

\section{Person in Context}

As discussed in the previous chapter, well-being is deeply embedded in the individual's relations to others and the social environment; however, understanding the person in context is not an easy task. As Cole (2003) argues, context is a slippery concept, notoriously polysemous. Trickett (1996) also claims that there is a diversity of contexts: social institutions, norms, policies or culture in general. Thus, clarifying the concept and the level of analysis are challenges for social scientists. Trickett argues that community psychology makes a unique contribution. In this chapter, sociological and psychological literature will be drawn upon to examine how the issue is viewed.

\subsection{Sociological Tradition}

There has been a long sociological tradition examining how social conditions influence people. In recent decades, micro-sociology theory has posed a great challenge to the macro sociology, leading to debate over the primacy of structure or agency on human thought and behaviour. Some sociologists, however, try to bridge the gap. A brief review of the sociological literature follows.

\subsubsection{Classical theory}

Durkheim, a foundational theorist, strongly believed that society is independent of individuals as it provides constraints to human action (Cheal, 2005). The analysis of social 'facts' is about function in relation to society as a whole, rather than it being reduced to individual and psychological explanation. For example, in explaining the social phenomenon of the division of labour, Durkheim (1964) argued against attributing it to individual characteristics as some economists did. Instead, Durkheim argues that it should be understood as the product of society. In his classic text Suicide, Durkheim (1952) argues that suicide, as an apparently highly individualist act, is deeply rooted in anomie. Conversely, in a cohesive society, people find mutual support as they exchange ideas and feelings. To Durkheim, "psychological facts are the results of social facts, not the reverse" (Cheal, 2005, p. 12). 
Like Durkheim, Marxists highlighted the determining role of society over individuals, particularly through economic means and the production of material life (Haralambos \& Holborn, 1995). Although Marx also realized that history is created by humans, he argued that social relations, the most important of which are economic relations (in his terms, the relations of production), are so determining that they nearly shape, if not control, everything in the society (Cheal, 2005, p. 88). Unless one becomes aware of the conflict between classes and strives for change, social relations sustaining the status quo will continuously and deeply embed the social structure, thus influencing the people. Thus, as he laments, "It is not the consciousness of men that determines their existence, but their social existence that determines their consciousness” (Marx, 1970, p. 21, quoted in Cheal, 2005, p. 88).

In contrast to Durkheim and Marx, Weber placed more emphasis on understanding the meaning people gave to their social behaviours. In his classic study of the emergence of capitalistic society, Weber (1958) tried to demonstrate the importance of the Protestant ethic in shaping individual action, finally leading to societal change (Giddens, Appelbaum, \& Duneier, 2005; Haralambos \& Holborn, 1995). Instead of having an independent structure 'out there', Weber believed that social structure arises from human actions and beliefs. Although his approach has been criticised as reducing social forces to individual action, his interpretive understanding of human action emphasises the importance of culture, not just the structure, of the society.

Generally speaking, the classical theory of sociology explains individual and societal operations according to rules embedded in the macro level and social structures.

\subsubsection{Micro-sociology}

Compared to the macro structure of classical sociological theory, micro-sociology focuses on human agency and examines how social systems can result from the microinteraction of individuals (Cahill, 2004; Collins \& Collins, 1994). It opposes the predetermined nature of society proposed by the classical functionalist or conflict theory. Instead, it "upholds the fluidity and meaningfulness of humanism” (Collins \& Collins, 1994, p. 243). Robert (2006) also regards micro social theory as a cluster of approaches unified by a common concern with human agency and the construction of meaning. The approaches aligned with this perspective include symbolic interactionism, social 
phenomenology and ethnomethodology (Knorr-Cetina, 1981; Roberts, 2006). Several theorists will be now highlighted because their work is beneficial to our later discussion.

Mead (1934) proposed the ideas of "I", “Me”, and “Generalized Other” to fully understand the concept of self. Instead of viewing the self as completely determined from the outside, Mead highlighted the reflexivity of human agents (Collins \& Collins, 1994). As Mead argues, there is an "I"-- a spontaneous self -- which actively responds to the social situation. Nevertheless, as he maintains, there is also a "Generalized other" -external-seeming rules -- that provides the abstract, non-specific audience on which the "I" can act and create thinking. As a result, people can have multiple "Mes"-- the derived self -- in different kinds of social relationships. Thus, to Mead, the self is derived from, but not determined by, the social experience and reality.

Building on Mead's idea, Blumer (1969) further emphasized the spontaneity and indeterminateness of social reality and develops his version of symbolic interactionism (Collins \& Collins, 1994). From Blumer's perspective, social reality is not a structure that is pre-determined, but a series of joint actions and processes socially constructed by the actors involved who undergo continuous negotiation and exchange of perspectives. Given the stability of the form, that does not mean it will not be different at other times. As Blumer argued, the existing patterns of social life "exist and persist only through the continued use of the same schemes of interpretation", which are "maintained only through their continued confirmation by the defining acts of others” (p. 67). However, once the interpretation sustaining them is undermined or changed, the same patterns of behaviours will rapidly collapse. Therefore, symbolic interactionism provides considerable space for human agency because people can infuse various meanings. Although the social environment may influence people's interpretation process, it has no determining role on human action (Blumer, 1969).

Influenced by Mead and Blumer, Goffman (1959) focuses on face to face interactions. In The presentation of self in everyday life, Goffman (1959) argues that people meticulously follow the ground rules of communication enabling smooth interaction and the maintenance of each other's "face". For example, civil inattention is a common strategy used by passers by. Wearing a white lab coat is the tactic medical persons use to display their professional image. Goffman's studies of the micro interaction revealed many neglected rules of human behaviour, but also helped him to 
build his conception of social order. As he understood, social order is "a set of ground rules which facilitate the pursuit of individual purposes in social situations, with the minimum of trouble for all concerned” (Cheal, 2005, p. 149). Thus, we can say that Goffman was also concerned about the structure, not the structure of society, but the structure of the social interaction.

In short, micro-sociology focuses on the meaning making process of individual and interpersonal relationships and how they are derived from the social interaction.

\subsubsection{The third position}

To resolve debates between micro and macro, or structure and agency, scholars have proposed innovative ideas, although whether these resolve the dilemma is debatable. Two of them are Giddens and Bourdieu.

Giddens (1984) proposed the structuration theory which eschews emphasizing either the structure or the individual. Instead, the theory focuses on regular social practices and actions (Giddens, 1979, p. 66) -- situated activities of human agents -which are influenced by both structure (such as rules and resources) and the human agent. Instead of viewing individuals as just passively imprinted by society, Giddens argues that individuals actively use their practical consciousness to draw on tacit knowledge, embedded in social activity, in human action. Nevertheless, only through the reflexive process of conduct can people verbalize and describe their acts and reasons behind these acts, to attain discursive consciousness, or to react to those acts. Otherwise, human agents are bounded and constrained by day-to-day life, which is reproduced by institutionalized structure (p.282). Therefore Giddens proposes the idea of duality of structure; that is, structure is both the medium and the outcome of human action. Social structure both produces and is produced by human action.

Compared to Giddens’ focus on structure, Bourdieu (1977) proposes the notion of habitus, around which he develops a theory of action. According to Bourdieu, habitus can be understood as a system of acquired patterns of perception, thought, and behaviour. These systems of acquired patterns of thought and practice are developed in the process and practice of responding to the demand of the objective social world (in his term, field). Habitus is not innate, but socially conditioned. People in the same field may have different habitus, because of the different positions they are in, the different previous 
experiences they have had, and the different capitals (see 3.3.1 for detail) they have acquired to respond to the challenges and demands of the objective world. For example, in the same classroom (field), different students will develop different patterns of thought and behaviour (habitus) to respond to the social challenge, according to the previous experience, social position, or resources (or capital) they have. Therefore, people may have different habitus, and the habitus acquired is socially and economically conditioned. Through the habitus, the culture and social structures are internalized in the individual or group experience. As argued by Maton (2008), the concept of habitus is anything but clear, but the concept may resolve the tension between the objective and subjective, and agency and structure (Cheal, 2005).

In summary, various sociological traditions -- classical theory, micro social theory, or the approaches of Giddens and Bourdieu, highlight the crucial role of social conditions in human action. Despite the fact that the room given to human agency vary.

\subsection{Psychological Tradition}

Compared to sociologists, psychologists seldom explore how the larger social condition can relate to human psychology; the majority of psychologists focus on internal and dispositional explanations of social behaviour (Fiske, Kitayama, Markus, \& Nisbett, 1998). As a result, situational and contextual factors are often disregarded. There are some psychologists, however, who do strive to understand individual behaviour in the social environment.

\subsubsection{Barker's Behaviour Setting Approach}

Barker, a pioneer in ecological psychology, extensively examined the relations between the environment and behavioural outcomes of children in two towns in the USA and England. He recognized that the traditional psychological approach, taking children as the unit of analysis, failed to provide sufficient insight and explanation. He took behavioural setting as the unit of analysis to explore environmental influences on behaviour. According to Barker, behaviour setting is a small scale system in which patterns of behaviour happen between human and nonhuman components, bounded within a temporal and spatial coordinate. For example, worship services are scheduled regularly in a particular place at specified times. Their components include pastors, choirs, 
congregation, pews, hymn books, bibles or other objects, which are arranged so that the services can run smoothly. Barker argues that the behaviour setting is independent of particular individuals, and each specific setting has certain scripts to direct people’s patterns of behaviour (Barker \& Schoggen, 1973). Moreover, behaviour settings are self regulating. Any “deviant” behaviour will be corrected so that appropriate behaviour patterns are maintained (Wicker, 1991).

The most significant contribution of behaviour setting is the 'manning theory'. Although any particular individual is not a concern to Barker's theorization, the number of people is still critical, because every setting requires a certain number of people to fill essential roles. In his studies (Barker \& Schoggen, 1973), Barker found that in settings with differing numbers of inhabitants, there are differing impacts on the setting itself, behaviour, and the psychology of participants. For example, people in a setting with fewer human components are generally engaged in a greater number and wider range of activities. They are more likely to take a leadership role and to be involved in more difficult and important actions. As a result, they may regard themselves as being more important. People in this kind of setting, however, may be simultaneously burdened with responsibilities beyond their control, so may feel greater insecurity. Conversely, people in overpopulated settings may experience greater competition and isolation within the settings. Nevertheless, overpopulated settings may be rich in resources and more diverse to suit different kinds of people.

Despite its stimulating and heuristic conceptualization and methodology, the widespread use of Barker's behaviour setting theory is still limited in psychology. One of the major reasons is its primary focus on environmental setting at the expense of concern for the individual. Another reason is its tedious, if not exhaustive, description survey methodology which is difficult for researchers to use. Wicker (1991) later expanded Barker's theory by acknowledging that the behaviour system itself has its own life cycle rather than having a "given" fixed entity; the motive and cognition of members within the setting still matters in the formation and change of the setting; and in the broader context, cultural and economic condition, for example, influence the immediate behaviour setting in a dynamic way. Most critically, Wicker argued that a behaviour setting is socially constructed, rather than being a pre-existing reality proposed and painstaking researched by Barker. However, Barker’s contribution is remarkable for he brought psychological 
studies back to people's natural setting. He also reminded the field of the crucial role of environment in human development.

\subsubsection{Bronfenbrenner's ecological approach}

In addition to immediate setting, Bronfenbrenner (1979) embraces both the individual and the environment, and puts various components in a system perspective, focusing on their interconnection and their aggregate impact on human development. Instead of focusing only on the immediate environment, like Barker, Bronfenbrenner proposes a comprehensive ecological model showing how an individual is embedded in, and influenced by, multi-level systems (micro, meso, exo and macro). In his conceptualization, the microsystem is the immediate setting where individuals interact with others face to face and where there is complex interrelation ( such as families and classrooms). The mesosystem is about interactions between microsystems where the developing person participates. The exosystem is the setting that is equally influential to individual development, but without the individual's direct involvement. For example, to children, the family school relationship is the mesosystem, while the parent's workplace is the exosystem. The macrosystem is the dominant culture and subculture where all socio-political and economic factors intertwine, providing the backdrop for all levels.

In his later explication, Bronfenbrenner (1995) argues that personal and environmental characteristics exert influence indirectly, through their impact on what he called proximal processes. Two propositions sum up his theory that emphasise the person, environment (both immediate and remote), and especially their joint interaction on the proximal process involving individuals.

Proposition 1: Human development takes place through processes of progressively more complex reciprocal interaction between an active, evolving biopsychological human organism and the persons, objects, and symbols in its immediate environment. To be effective, the interaction must occur on a fairly regular basis over extended periods of time. Such enduring forms of interaction in the immediate environment are referred to as proximal processes. Examples of enduring patterns of proximal process are found in parent child and child 
activities, group or solitary play, reading, learning new skills, studying, athletic activities, and performing complex task.

Proposition 2: The form, power, content and direction of the proximal processes effecting development vary systematically as a joint function of the biopsychological characteristics of the developing person; of the environment, both immediate and more remote, in which the processes are taking place, and the nature of the developmental outcomes under consideration. (Bronfenbrenner, 1995, pp. 620-621)

Bronfenbrenner (1995) argues that 'who' the children are, what the environments are, and their joint interaction, affects the proximal processes in which children are embedded, and play a role in enhancing or inhibiting child development. However, as he argues, the operation of proximal processes and methods of study are less familiar to researchers. Furthermore, Bronfenbrenner also explores the temporal dimension in relation to the environment: historical events in the developmental process, and the timing of those historical events, all shape and create the environment that individuals are embedded in. Therefore, Bronfenbrenner expanded his person-context model to propose the process-person-context-time (PPCT) model of human development (Bronfenbrenner, 1995). Bronfenbrenner not only took ecology seriously, but also provided a comprehensive framework to make sense of the complex relationship between environment and human development.

\subsubsection{Sarason's Psychological Sense of Community}

In contrast to Barker's focus on the immediate context and Bronfenbrenner's multi-level ecological system, Sarason (1974) explores the social context and community influence by focusing on individual psychology. Facing disintegrating contemporary society, he proposes the concept of the psychological sense of community (PSOC) as the overarching criterion of community psychology and argues that for community intervention to be effective, the individual's feeling of being a part of a larger structure should be the prime focus of researchers. 
Despite being illuminating, it took the work of McMillan and Chavis (1986), more than ten years later, to develop it. McMillan and Chavis proposed that PSOC is defined by four elements that together form a feeling of belonging, mutual care, and shared identity. The four elements are membership, influence, integration and fulfilment of needs, and shared emotional connection.

Membership is about who belongs to the community and who does not. Membership unavoidably generates boundaries against “deviants”, to protect insiders” emotional safety. Personal investment opportunities are also important to being a member because through such investment, identification and common symbol systems can be formed within the community.

Besides being a member of a community, influence is an important dimension for a person to have a sense of community within a setting. The individual influences the community, and the reverse is also true. The more cohesive the community, the more influence the community has on members, eventually resulting in conformity. Since influence can be mutual, between members and the community, those who are more influential may eventually become leaders of the community.

According to McMillan and Chavis, the integration and fulfilment of members' needs is also important; if the community experience is rewarding for members, they will be more involved in the community, resulting in the strengthening of the community. McMillan and Chavis suggest that effective reinforcers of community include status of membership, success of the community, and the competence of other members, which all contribute to satisfaction within the community.

Last, but not least, McMillan and Chavis suggest that to be a part of a community, individuals should have shared emotional connection, which is partly based on a shared history, or history identified with. To attain this, contact opportunities are primary, and the quality of interaction is very important. Moreover, the more important the shared events, the stronger bond the people can develop with the community. The extent of importance not only depends on the event per se, but is also determined by the extent people invest themselves, in terms of time, money or emotional risk. Therefore, all opportunities which offer the experience of honour, humiliation or common spiritual bonding, matter and contribute to the sense of community. 
After McMillan and Chavis’s major work, scholars have applied the concept to various types of groups or communities, including neighbourhood (Brodsky, 1996; Brodsky, O' Campo, \& Aronson, 1999), immigrant groups (Sonn, 2002; Sonn \& Fisher, 1996), religious community (Miers \& Fisher, 2002), young people (Pretty, 2002), gay people (Proescholdbell, Roosa, \& Nemeroff, 2006), and an international community of interest in science fiction (Obst, Zinkiewicz, \& Smith, 2002). ${ }^{1}$ A sense of community also has been shown to have positive influence on various outcome variables, such as wellbeing (Davidson \& Cotter, 1991; Obst \& Tham, 2009), and participation (Chavis \& Wandersman, 1990; Davidson \& Cotter, 1989). These studies demonstrate that the notion of a sense of community is useful and helpful to understand not only the locational, but also the relational dimension of the setting and to the psychological dimension. Despite criticism of reliance on personal perceptions, PSOC is important in studying community as it takes into account the community context.

\subsubsection{Vygotsky's understanding of culture}

Vygotsky (1978) also takes subjective perception seriously. While

Bronfenbrenner provided a comprehensive framework of human development, incorporating both individual and multi-level layers of environment, Vygotsky was one of the few, if not the first, psychologist to explore the broader environment -- culture-built into individual minds (Cole \& Scribner, 1978). By observing children’s behaviour in the experimental settings, Vygotsky discovered that children not only use symbols (particularly speech) in their actions, but also use speech to help complete an action, particularly difficult tasks. In other words, by using or not using speech, children complete actions differently.

Vygotsky argues that the use of signs affects perception, attention and memory, resulting in higher psychological functioning. The use of signs provides greater independence from the concrete visual situation, and also greater mastery over actions and responses to external stimuli. Therefore, instead of responding impulsively and spontaneously like animals, humans are able to plan, think and mentally process before action are taken, by the use of words and speech. Human action progresses from the elementary stimulus-response cycle to complex and mediated acts, the basis of all higher

\footnotetext{
${ }^{1}$ Interested readers can refer to Fisher, Sonn and Bishop (2002) for further review.
} 
psychological processing. Vygotsky (1978) argues that "the most significant moment in the course of intellectual development, which gives birth to the purely human forms of practical and abstract intelligence, occurs when speech and practical activity, two previously completely independent lines of development, converge” (p. 24). Humans are different from the higher animals through the ability to use symbols and language (in addition to tools), enabling more complex and higher level psychological processing. Vygotsky (1978) argues that there are different stages of sign operation. In the early stage of development, children still require external signs as auxiliary psychological instruments. In the later stage, adults can more readily transform external signs into internal signs that make the internalization process complete. Vygotsky notes that internalization involves “long series of developmental events” (p. 57), through interaction with others. Individuals also take time to transform the interpersonal process (including voluntary attention, logical memory and the formation of concepts) into an intrapersonal one, without guarantee of success. According to Vygotsky, child development is culturally mediated and occurs on two levels through the internalization process: "first, on the social level, and later, on the individual level; first between people, and then inside the child” (p. 57).

Vygotsky was not solely concerned with the sociogenesis of mind (Cole \& Wertsch, 2002) as he also emphasized the active construction of the individual; however, he does view human development (including thoughts, actions, and experiences) as being culturally mediated, if not culturally bounded. As Nicholl (2006, last para.) notes, "what we learn will depend on the psychological tools available to us and which tools are available will depend upon the culture we live in.”

Vygotsky's meticulous handling of signs helps bridge the gap between understanding culture and the human mind. His understandings of culture and its relationship to human development provides ideas and insight to subsequent psychological theory in the area of cultural psychology (Cole, 1996; Carl Ratner, 2006) and developmental psychology (Rogoff, 2003).

In summary, the various psychological perspectives described above are bold attempts to involve a broader context in the study of individuals. Countering a sole concern with societal conditions without room for human agency (as with some 
sociology), the psychological tradition tends to retain the human role, even as it acknowledges the influence of society.

\subsection{Other emerging perspectives}

Compared to sociological and psychological disciplines, the disciplinary boundary in the following emerging perspectives is rather blurred. Moreover, the focus is on multilevel understanding of social phenomena and change.

\subsubsection{Social Capital}

"Social capital” is a hotly debated concept that involves social networks and social relationships (e.g., trustworthiness) and the broader social context (such as economic development). The concept has implications for public policy and has attracted much attention in academia and more widely (Kwame McKenzie \& Harpham, 2006b). The major proponents of the concept are Boudieu, Coleman and Putnam, even though these scholars do not form a consensus around the concept.

In Bourdieu's (1986) understanding, capital are resources possessed to do certain things. Social capital, "is the sum of the resources, actual or virtual, that accrue to an individual or a group by virtue of possessing a durable network of more or less institutionalized relationships of mutual acquaintance and recognition” (Bourdieu \& Wacquant, 1992, p.119, quoted in Cheal, 2005, p. 157). Therefore, people in different positions may possess differing amounts of social capital because of the various social ties they acquire. Maintaining social ties takes time and requires economic resources or knowledge of interpersonal relationships. Social capital is also largely determined by possession of other capital, such as economic or cultural capital. According to Bourdieu, although social capital seems like an individual's property, it is actually deeply embedded in, and dependent upon, the community resources.

Coleman (1988) also regards social capital as “a resource for action” (p. 95), arguing that higher levels of social capital within and outside the family, in the form of obligations and expectations, information channels, and social norms, can help create human capital. Coleman notes that social capital arises in a social structure. Therefore, to Coleman, social capital is a valuable conceptual tool, involving the economic principle of 
rational action in analysis of the social system, without discarding social organization in the process.

Like Bourdieu and Coleman, Putnam (2000) also explores the value of social networks. Putnam puts more emphasis on intangible connections between individuals: mutual trust, reciprocity and social norms. In addition, compared to Coleman and Bourdieu who take a neutral stance towards the concept, Putnam uses the concept in a more positive notion. In his popular book Bowling Alone (Putnam, 2000), Putnam argues that many social ills are caused by a lessening of individual participation in society, leading to a decline of social capital. Although Putnam's concern with social capital is on the community level, his definition of social capital focuses on trust and relationship, rather than community resources. This creates the risk of reducing the concept to an individual attribute, without acknowledging that trust and relationships are deeply embedded in the environment.

Compared to Putnam's focus on the effects of social capital on the public good, the social network theory of social capital discussed by Lin (2001) is centred on the use of resources embedded in social networks that affect individual action. In Lin's conceptualization, people have two kinds of actions: instrumental and expressive. Instrumental action is about gaining resources and attaining certain goals (like finding a job), while expressive action is about maintaining and preserving resources (like sharing feeling with others). He argues that those at the top of the social hierarchy tend to display expressive action because less people with better resources are above them. Therefore, the motivation for instrumental action is reduced, and they choose to interact with others from similar demographic backgrounds to maintain valued resources (following the homophilous principle). In contrast, those in the middle of the social hierarchy tend to display instrumental action because of their potential access to those with better social capital. So they tend to interact with others from diverse backgrounds to increase their access to better resources - they do not act according to the homophilous principle.

In summary, despite being still being a fuzzy concept, social capital reveals the importance of the intertwining relationship between individual action, social relationships, and broader social structure. 


\subsubsection{Systems Perspective}

The social capital theory is generally proposed by sociologists or political scientists, but the systems perspective appeals to scholars from diverse disciplines, including scientists, social scientists, educational and organizational researchers.

Systems perspective has a long history, but it became an area of study after the 1940s, drawing from the work of scholars from various backgrounds, of whom Bertalanffy, who proposed his general systems theory in 1950, is one of the prominent figures (Bertalanffy, 1968). The major idea of this perspective is the concept of a system within which component parts are linked together in a web of relationships. The whole and the parts as well the interrelationships all crucially constitute the system. These features apply to all kinds of systems. However, Boulding (1956) noted that there are various types or levels of systems that differ in the complexity of their parts and in the nature of the interaction between the parts. The more complex the system (such as a social system in comparison to a mechanistic system), the less constrained and deterministic, if not loose, the relationships are between the interacting parts. Within the complex system the effect of one component part over the other component part is multiple, and less predictable.

Recently, some community psychologists have applied the systems perspective to understanding social setting and other phenomena, especially focusing on system change (Foster-Fishman \& Behrens, 2007; Kelly, Ryan, Altman, \& Stelzner, 2000). Using system thinking and the perspective of organizational change, Foster-Fishman, Nowell, \& Yang (2007) proposed a framework to understand the system-part interaction and mechanism, in order to lever system change. In their framework, they suggested that system norms, resources, regulations and operations be further examined to reveal the root causes and bring about system change. These four parts of the system also involve both participants and environmental setting.

\subsubsection{Organizational studies}

Similar to the system perspective, organizational researchers have long realized that organizations are difficult to comprehend because they are complex, deceptive and always ambiguous (Bolman \& Deal, 2003; Scott, 2003). Besides the structural dimensions of organizations, the human components within organizations can make 
understanding organizations more difficult. Further complicating the issue is the emerging consensus that organizations are not merely closed systems isolated from external influence, but open systems intertwined with the broader environment (Scott, 2003; Scott \& Davis, 2007). Scholars therefore regard social organizations as complex and loosely coupled systems (Buckley, 1967; Scott, 2003). Instead of using a single lens, multiple perspectives are adopted to study these complex issues.

The structural frame proposed by Bolman and Deal (2003), or the rational system perspective suggested by Scott (2003), can be viewed as among the more popular perspectives adopted. The rational system views organization as a machine in which the established goals of the organization guide and direct the role and behaviour of individuals and the structure of the organization. To maximize the effectiveness, a formal structure should be well designed so that it not only formulates and facilitates expectation and exchange between participants, but also prevents confusion and inefficiency. Therefore, formalization and goal specificity are important in this perspective, while individual differences are seldom of concern.

In view of the limitations of the classic model, the human dimension of organizations - or natural system in Scott's (2003) term - is gaining more attention (Bolman \& Deal, 2003; Scott, 2003). Scott observed that, since the classic Hawthorne studies, more researchers suggest that certain needs, especially need related to participants in organizations, have to be fulfilled if the organization is to survive. Therefore, while individuals provide labour for the organization, financial rewards or career advancement opportunities have to be offered in return. The rewards, however, are not necessarily financial or tangible in nature. Interpersonal relationships, motivation, and opportunities for participation by employees are some of the other factors contributing to individual satisfaction, crucial to the success or failure of the organization. The challenge faced by organisations is to balance and align individual needs with collective goals.

Besides the structural and human resource frames, Bolman and Deal (Bolman \& Deal, 2003) also discuss the political and symbolic frames. These perspectives align with conflict models and organizational culture perspective. The political frame is concerned with power and the political game played by different parties in the organization, while the symbolic frame is about cultural norms or expectations shared and created within an organization. As well as collective (or macro) goals and individual (micro) differences, 
these political or cultural perspectives provide additional insight into organizations, taking the meso level of organization into consideration.

Despite advance from mechanical to organic systems, organizational scholars still find the above perspectives too simplistic. Instead of viewing organizations as closed systems unaffected by the external environment, this perspective perceives organizations as open systems (Scott, 2003), both affected by and affecting the broader environment beyond the organizational level. Since the environment is always changing, organizations are seen as amorphous with extraordinary high complexity and variability in their individual but interdependent parts. That also explains why the contours of organizational theory shifts from viewing organizations as a structure to viewing organizations as a process (Scott \& Davis, 2007; Weick, 1979). In other words, organizations are no longer seen as fixed entities unaffected by internal dynamics or the external environment. Rather, they are always changing and interacting, both internally with the participants and within the system, and externally with the social world in which organizations are embedded.

Therefore, organizational studies seem to suggest that individual differences, organizational culture, and goals, as well as the wider environment, are all closely related and intertwined. To understand organizations, all these aspects have to be examined.

\subsection{Summary}

From this short review of literature, two major conclusions can be drawn. Firstly, as Trickett (1996) argues, there is a diversity of contexts which have impact on people, whether regarding social structure, network, economic system, cultural system, immediate setting, community, social or cultural environment, or organization. Secondly, as argued above, the environment and the individual cannot be easily separated. In other words, person and context are intertwined with each other (Cole, 1996; Rogoff, 2003). From the literature from across various disciplines, it is found that the sharpest and clearcut structure-agency division is breaking down; there is a concurrent convergence around disciplines traditionally responsible for understanding phenomena at different levels. Sociology, normally concerned with the macro level and social structures, now focuses more on understanding individuals who compose society; while psychology, which traditionally cares about the psyche of individuals, increasingly focuses on the ecological and social context and how it relates to the intrapsychic functioning. The recently 
emerging perspectives also adopt a more interdisciplinary and holistic approach that involves both the whole and the parts, as well as their interaction.

All these efforts remind us that to fully understand human behaviour, or social phenomenon (including well-being), we have to attend to both the person and the environment (Lewin, 1951; Riger, 2001). Without addressing the ecological context, psychologists may easily personalize the issue without focusing sufficiently on the individual. People may be perceived as being passive recipients of environmental influences, rather than active agents dealing with and influencing the environment (Riger, 2001). By understanding subjectivity, and how it is deeply embedded in a multi-layered social environment, we can avoid oversimplification of the issue and be able to provide intervention appropriately.

Nevertheless, there are also great challenges in understanding individuals in context and taking the ecology seriously. The challenge is not only conceptual, but also methodological. As mentioned previously, context is a slippery concept. One solution is to find a proper unit of analysis (Van der Veer, 2001) for each specific study, so that the phenomenon can be understood clearly. As regards methodology, as noted by Bronfenbrenner (1995), psychologists are still unfamiliar with the method that enables them to study people in context (especially the interplay between the person and the environment and the complex psychological process).Some social scientists tend to regard the environment as independent and as a factor or variable external to the individual; however, to understand people in context we cannot escape the intertwined relationship between people and the environment.

Spirituality and the religious community will also be a focus, when examining well-being. The influence of the broader social environment on religion cannot be ignored, but it is not the primary focus of this study, despite the social and historical context of religious setting being provided. Moreover, spirituality and religious community will not be examined separately because both levels influence each other; studying them in isolation hinders full understanding of the issue. Giddens (1984), and Hays (1994) both argue that structures can both constrain and enable human actions. Likewise, religious communities can both constrain and enable the development of spirituality. Furthermore, as argued in the previous chapter, well-being is deeply embedded in the social environment; well-being derived from spirituality is also deeply 
embedded in religious communities. In the following chapters, religion's impact on the individual is examined. In Chapter Four, we discuss the foundational concept of religion. In Chapter Five, we will explain how religion affects people's well-being at multiple levels. 


\section{Chapter 4}

\section{The Conceptualization of Religion}

What is religion? How is it measured? Can religion be studied scientifically and from an outside perspective? Is a science of religion possible? These are all thorny questions that scholars of religion have studied and that are not easy to answer. In the following section, the debate about the possibility of a science of religion will be presented and then different conceptualizations of religion will be explored in detail. At the end of the chapter, an integrated approach used in the present study will be presented.

\subsection{The Insider-Outsider Debate}

Religion is a long lasting human phenomenon and there are traces of it in ancient texts or even prehistoric burial offerings. There is the lay understanding of religion, but scholars find it extraordinarily difficult to reach a consensus on what religion really means. Comparative religious scholar Wilfred Cantwell Smith (1962/ 1991) finds the notion of religion problematic and heavily biased by the western influence. Smith claims that, despite early indications of the relationship between God and man, the term religion was later meant a system of beliefs, because of the institutionalization of religious faith, which was indeed abstract and nonexistent. He concludes that the term religion has multiple meanings, including personal piety, the ideal or empirical form of an overt system of beliefs, practices, values; or religion in general (pp. 48-49). It seems that religion is a multifaceted phenomenon and the debate over religious may results from different aspects of the same phenomenon referred to. One of the key issues surrounding the debate is the insider-outsider perspective; or, the reducibility or not of religion.

\subsubsection{An irreducible account of religion}

One of the major proponents of the irreducible account of religion is Mircea Eliade, who argues that religion should be understood on its own plane of reference, and cannot be reduced to other secondary aspects like historical, psychological or sociopolitical accounts (Eliade, 1961/ 1999). Otherwise, the essence and autonomy of religious phenomena will be lost. Instead, Eliade argues that scholars of religion have to 
understand the meaning of religion, on top of knowing the historical, socio-economic, or political context. Using artwork as an analogy, Eliade illustrates why understanding a particular country in a period of time, its corresponding theology and philosophy, and being familiar with the artist's life, is still insufficient to comprehend the artistic value of art work. Religious phenomena too can be understood by its meaning as an autonomous creation, without regressing to constituent elements; however, Eliade admits that religious phenomena cannot be isolated from historical and socio-cultural context.

\subsubsection{In defence of the reducible account of religion}

Opponents of the irreducible account of religion argue that an insider perspective accepts the believer's point of view, without justification, and argue outsider perspectives or reducible accounts of religion are not only valid, but necessary. Segal (1983/1999), in his article In Defence of Reductionism, argues that to non-believers, including agnostics, reductionistic interpretations of religion enable understanding (or even appreciation of) religious phenomena, and they are unable to hold or identify with the religious interpretation claimed by the believers. Moreover, Segal insists that the reductionist interpretation is not necessarily inadequate to understand religious phenomena merely because it is reductionistic. Rather, it is the scarce amount of study that makes the reductionistic interpretation not adequate for the truth claim. Segal believes that reductionist interpretations "have failed not because they have been reductionistic at all but because they have not been reductionistic enough” (p. 156).

\subsubsection{Agnostic stance}

Contrasting with the insider-outsider dichotomy, some scholars sidestep the notion of truth in religion and take a neutral stance on the metaphysical issue; they view religious phenomena as a social fact, but understand innermost religious experiences as inexpressible and unobservable. Wiebe (1999) argues that religion can be studied scientifically by examining “embodied” experience "in a diversity of observable, intellectual, practical and social expressions” (p. 266). Although religious experience is important to understand religious phenomena, religious understanding is not the only way, exclusive to outsiders, to make sense of religious phenomena. Scholars of diverse disciplines have valid observations to make about religious phenomena, if it is studied 
rigorously. Nevertheless, as Wiebe argued, it is reductionistic, but is not metaphysical reductionism. Rather, it is the reduction of the religious experience to some religious expression that is observable, describable and researchable.

We can now make sense of the varieties, if not the complexities, of conceptualizations of religion and subsequent debates. Different approaches focus on different aspects of religion: the innermost religious experience, expressible religious belief, or even religious organization upholding religious phenomena.

\subsection{Defining Religion}

Religion has both individual and social facets, spans geographical and temporal space; the conceptualization of religion may differ according to different disciplinary foci. As expected, a consensus on a definition of religion is far being accomplished. What is common is the intricacy of religion. Smart (1998) argues that there are many traditions and sub-traditions of religion and it is more practical to define what $a$ religion is, rather than what religion is in general (p. 12). Some scholars (e.g., P. C. Hill \& Pargament, 2003; McGuire, 2002) also suggest that it is better to view the definition of religion as strategies within specific investigations (e.g., in psychological studies concerning religion, and with respect to health, religion may be considered a specific kind of motivation or individual attribute), rather than as truth. A brief review of the different conceptualizations of religion will be presented before the author's position is outlined.

\subsubsection{Spirituality and religion}

Before presenting different conceptualizations, terminology can be defined. Particular attention can be paid to notions of spirituality. In current usage, spirituality concerns the individual and the personal; religion involves the institutional dimension. Another usage, unfortunately, involves the polarization of spirituality as 'good' and religion as 'bad' (Pargament, 1999). As Pargament (1999) argues, in the long tradition of the psychology of religion, religion embraces both the individual and the institutional, the substantive and functional, and the good and bad. In the present thesis, the author has no intention to draw a clear line between the two concepts or makes judgments. Following Pargament (1999) and Dorr (2004), religion will be used as an umbrella term encompassing spirituality as the core and essence of religious life. Religion is used as a 
general term including individual and/or institutions. When necessary, to explicitly differentiate between the two levels, spirituality and religious community are used.

\subsubsection{Phenomenological tradition}

Smart (1996, 1998), from the comparative and phenomenological tradition, categorizes religion in seven major dimensions: practical and ritual; experiential and emotional; narrative or mythic; doctrine and philosophy; ethical and legal; social and institutional; and material. The practical and ritual dimension concerns the faith practice of believers, and the experiential dimension looks at the development of people's feelings of the sacred. The narrative and mythic dimension is about the story and mystery embedded in religion. The doctrinal and philosophical dimension concerns the intellectual reflection. The ethical and legal dimension concerns the laws and ethical practices that a religious tradition incorporates into its social composition. The last two dimensions suggested by Smart concern the incarnated form of religion: religious organizations and various material forms of religion, such as buildings, art, icons, and holy places..

Smart's $(1996,1998)$ categorization provides a comprehensive mindmap of the varieties and richness of religion, without sticking to a specific type of religious tradition. Smart's dimensional approach also reminds us that religion can be understood from many perspectives, spanning various disciplines, such as psychology, sociology, anthropology, theology, history, and linguistics.

\subsubsection{Psychological tradition - Religion as subjective religiosity}

Psychologists focus on internal attributes, and use various dimensional approaches to understanding religion as subjective religiosity.

\subsubsection{Religious orientation}

In psychology, the classic, empirically useful definition of religion is contained in the work of Allport and colleagues (Allport \& Ross, 1967), who compared people who "live their religion" (intrinsically oriented) with those who "use their religion" (extrinsically oriented). They found that intrinsically religious people are more mature in faith, less prejudiced and more helpful towards others. Extrinsically religious people are 
more prone to prejudice and less helpful. Batson and Ventis (1982) attempt to revise the model, adding the "quest” dimension: an individual's openness and flexibility towards certain religious beliefs. However, religious orientation has been widely used in the psychology of religion.

\subsubsection{Religious experience}

Other psychologists try to understand religion from its innermost experience, including Otto’s (1917) “numinous” experience, James’s (1902/ 1960) varieties of exotic religious experiences and Pratt’s (1924) “milder type” religious experiences. In addition, other transcendent, mystical, aesthetic, charismatic, paranormal or near-death experiences are also not uncommon in understanding different kinds of religion (Argyle, 2000). In The Handbook of Religious Experience, Hood (1995) and colleagues provide us with a rich account of religious experience related to various psychological theories, including depth psychologies, developmental and cognitive theory, or other more specific psychological perspectives: role theory, attribution theory or attachment theory. Despite different foci, as Argyle (2000) notes, religious experience is crucial, and without it, there is no religion at all.

\subsubsection{Religious belief}

Another major dimension of religion is religious belief. Argyle (2000) and Silberman (2005) both argue that religion as a belief or meaning system is unique because it is not just the assent to certain kinds of verbal propositions, but grounded in powerful emotions towards sacred objects, strong motivation, as well as commitment to action. In addition, religion is able to provide people with meaning in nearly every aspect of life and provide answers to life's deepest questions, as well satisfying the human need for transcendence. Religious belief, in theology, metaphor, religious stories or myth, not only involves experience of God or the divine, but also act as moral guidance, directing behaviour, or even whole lives. In 2005, a special issue of the Journal of Social Issues (Silberman, 2005) explored how religion, as a unique meaning system, can enormously influence various aspects of people's lives. As argued by Park and Paloutzian (2005) and Silberman (2005), viewing religion as a meaning system provides a promising framework for researchers in psychology of religion to integrate their work. 


\subsubsection{Other measures}

There are other measures of religiosity, described in the work of Hill and Hood (1999). Church attendance and involvement are often cited, although they are not the only measures. Hill (2005) summarizes several recently emerging dimensions of religiosity. Hill and Pargament (2003) argue that church attendance, or general measures of religiosity, are useful to show the importance of religion and spirituality, but cannot explain how and why religion influences outcomes, such as health. They suggest that the measurement of religion largely depends on what other variables the researcher intends to relate with religion. For example, Hill and Pargament propose four promising concepts that are functionally related to physical and mental health: perceived closeness to God, religion as motivating forces, religious support, and religious struggle.

\subsubsection{Sociological tradition - Religion as groups or organizations}

Besides subjective religiosity, religious groups or bodies are also the critical area of concern in the study of religion, particularly in western society. In this area we should draw on the insights provided by sociology. Durkheim (1912/2001), a pioneer of sociology, argues that religion has a significant role in society, providing social integration, shared values and norms, which can direct behaviour. The social dimension of religion cannot be ignored. Although sociologist Glock (1962) describes religion in five dimensions - experiential, ideological, ritualistic, intellectual, and consequential but a more particular sociological contribution to the understanding of religion is the classification of religious traditions. Weber (1922, cited in McGuire, 2002) proposed church and sect to differentiate the mainstream orthodox and marginal religious bodies, and the concepts of denomination (Niebuhr, 1929) and cult were added to describe sects that become institutionalized, stabilized and accommodated to society, and those loose associations of persons with private religiosity (McGuire, 2002).

\subsubsection{Religious Factors}

Religious factors are also commonly used in sociological inquiry. Lenski’s (1963) classified American religion into four major categories: white Protestants, black Protestants, Roman Catholics, and Jews, noting that these various religious traditions are conducive to particular groupings of attitudes, values and behaviour (Wuthnow, 2004b). 
Following Lenski's work, the often used taxonomy of religious groups in the US include mainline Protestant, evangelical Protestant, black Protestant, Fundamentalists, Pentecostal, Catholic, and Jewish (Welch, Sikkink, Sartain, \& Bond, 2004; Wuthnow, 2004b). Their differing influences on social life has been studied (Welch, et al., 2004; Wuthnow, 1999). According to Welch et al. (2004), mainline Protestants tend to be open towards society, emphasizing outreach. Evangelicals, despite strong bonds within the church, still emphasize engagement with society. However, fundamentalists try to detach themselves, maintaining a strong boundary between the congregation and society. Pentecostal Protestants focus on individual religious experience. These different outlooks, according to Welch et al., have differing impacts on people’s trusting behaviour.

\subsubsection{Classification of denomination and congregation}

Other sociologists use specific dimensions to create religious groups for understanding. For example, the fundamentalist-liberal continuum is an often used dimension to classify denominations - fundamentalist, moderate and liberal - and is good at predicting various religious, social and political variables (T. W. Smith, 1990). McGuire (2002) observed that there are structural shifts in affiliation with official religion. The religious unit in which people engage is no longer the denomination, but local congregations or religious organizations. Various kinds of typological approaches are applied to congregations too (Becker, 1999; Carroll \& Roozen, 1990; Dudley \& Johnson, 1993; Roozen, McKinney, \& Carroll, 1984). Roozen et al., (1984) use two dimensions in relation to mission orientation -- whether the mission of the congregation is “this world” and "otherworldly”, and whether it is "member centered” or "publicly proactive” -- to derive four types of orientation. These orientations are: sanctuary, evangelist, civic and activist. The church with a sanctuary orientation tries to provide people with a sacred refuge from the world; the evangelist orientation focuses on converting people and preaching the gospel; a civic orientation tries to promote what is good in the world; and an activist orientation addresses the structural causes underlying suffering and injustice. Becker (1999) examines internal conflict in congregations and identified four fundamental congregational identities always in tension. A "leader congregation" is like an activist and mission focused community, the "community congregation” acts as a democratic body supporting members to pursue their societal 
ideal. The "family congregation” strives to provide members with close ties and a supportive environment, and the "house of worship” congregation provides religious goods and services to members. Moreover, using nine dimensions and cluster analysis, Carroll and Roozen (Carroll \& Roozen, 1990) derive six types of congregational identities within the Presbyterian Church, shown to be related to the tasks and programs that are available in the congregation.

The idea of classification and religious factors is important for two reasons. It provides another way to conceptualize religion, more compatible with religious context. Some also argue (McGuire, 2002; Wuthnow, 2004b), that classification or typology helps explore whether various religious types matter in social life or have a social impact.

\subsubsection{Anthropological tradition - Religion as a cultural system}

Although the psychological and sociological approaches take into account of both the individual and the religious setting, but standard survey approaches are criticized as being "extremely limited in their ability to tap the depth and complexity of each individual's religion and religiosity” (McGuire, 2002, p. 109). Belzen (1997) also argues that to really understand religious phenomena, the historicity of human subjectivity and cultural setting cannot be overlooked. Method and concepts drawn from other disciplines, like anthropology, are beneficial to understanding religion. Geertz (1973), one influential person in the discipline, views religion as a cultural system.

In contrast to sociological emphasis on material and structural components, Geertz (1973) argues that, to understand human communities, it is necessary uncover the “hidden attitudes and emotions” underlying social order: “culture” (Pals, 2006). Unlike other American pioneers, Geertz does not regard culture as only a kind of group attitude. Instead, he considers culture as an objective system of ideas, customs, meaning and symbols, governing the private emotions and behaviour of individuals.

Geertz agues that although culture is about the meaning and thought behind individual acts, it is not personal. On the contrary, it is shared in the community or society, otherwise the individual behaviour cannot be comprehended or understood. Moreover, culture is not just about meaning, it is also about the flow of behaviour and action through which "cultural forms find articulation” (Geertz, 1973, p. 17). Culture can be studied and articulated empirically and objectively. To reveal the culture of a society, 
Geertz advocates a method called "thick description" whereby researchers immerse themselves in the setting to discover the nuances and minute details of the context.

In his ethnographical study of the Java community, Geertz notes the role religion plays in influencing the values and meanings used to make sense of life. Geetz states:

"A religion is: (1) a system of symbols which acts to (2) establish powerful, pervasive, and long lasting moods and motivations in men by (3) formulating conceptions of a general order of existence and (4) clothing these conceptions which such an aura of factuality that (5) the moods and motivations seem uniquely realistic." (Geertz, 1973, pp. 90-91)

In this condensed sentence, religion is considered to be anything (object, event, ritual, gesture, action or image) able to pass on ideas. They induce aspiration, but religious symbols are public things that exist outside individuals (Pals, 2006). Compared to other cultural system like art, Geertz argues that religion elicits powerful motivations and moods, grounded in an ultimate explanation of the world assumed to be realistic and more real than anything else; a unique set of meanings intertwined with society and the individual human mind.

\subsection{An integrated Approach}

Acknowledging the positive and negative impact of religion on human society, psychologists increasingly strive to understand the psychology of religion so as to improve humanity and society (Allport \& Ross, 1967; Emmons \& Paloutzian, 2003; Paloutzian \& Kirkpatrick, 1995; Wulff, 1997 see the previous chapter for an overview). Nevertheless, what religion is about, empirically and conceptually, is still far from clear. Consensus is lacking among scholars. To advance the field, some problems encountered by previous study should be borne in mind.

Paloutzian and Kirkpartrick (1995) argue that many previous studies are methodologically and conceptually too simplistic, and more focused, sophisticated research informed by strong psychological theory is needed. Gorsuch (1984) notes that the goal of religious study is not to develop new measurement, but to study the impact of religious phenomena. He proposes using measurement paradigms with other less traditional approaches to develop more comprehensive understanding. Following Gorsuch, Paloutzian and others advocate the multilevel interdisciplinary paradigm to 
study religious phenomenon (Emmons \& Paloutzian, 2003; Paloutzian \& Park, 2005). Moreover, Pargament (2002a) and Maton (2001) argue that to fully understand the richness and complexity of religious phenomena, contextually based, multidimensional studies are needed to shed light on the multilayered nature of spirituality and religion. The dark side of religion needs to be noted, so as to examine the costs and benefits of religious life. As Pargament (2002a) argues, the fruits of religion can be bitter and sweet, depending on the kind of religion, the individual, the well-being being measured, the situation, social context, and the degree to which religious life is integrated into overall life. There are no simple answers. These issues form the basis of the present study.

Community psychologists Dokecki et al. (2001) undertook ten years of action research in a local parish, developed a "head-heart-hand" model of spirituality embracing both human and community development. They argue that spirituality is the pursuit of self-transcendence, going beyond the self, and relating to others, society, the cosmos and finally the Ultimate Other. In relating to the other, and finally the Ultimate Other, spirituality manifests itself in a form of bonding, reflection, and action; a communal experience of being together, reflecting on that relationality, and in action or service to strive for ultimate values. Dokecki et al. argue that balancing and integrating the three dimensions of spirituality (communion, reflection, and action) is essential for human and community development.

Realising the complexity of religion and the pitfalls of the simplistic methodology used in past research, a refined framework is suggested in the present study (see the Figure 1). The integrated approach involves subjective religiosity, religious typology, and cultural systems. The framework adopts Dorr’s (1984) and Dockeki et al.'s (2001) multidimensional aspects of spirituality because spirituality is integral to individual religious beliefs, practice, and experience within a spiritually based context, and manifests in personal, interpersonal and social dimensions. Spirituality (or subjective religiosity) is deeply embedded in specific historical and socio-cultural contexts; the religious typology and cultural environment of the congregation can strongly influence ways spirituality is manifested. The relationship between religion and well-being can be diverse and intricate depending on the kind of religion and well-being concerned, and the socio-cultural context. Religion not only can address people’s various religious needs (relational, intellectual and participatory), but also enhance well-being on various levels 
(individual, interpersonal and collective). It provides a context that facilitates and sustains development. We will now explore empirical findings concerning the relationship between religion and well-being. 


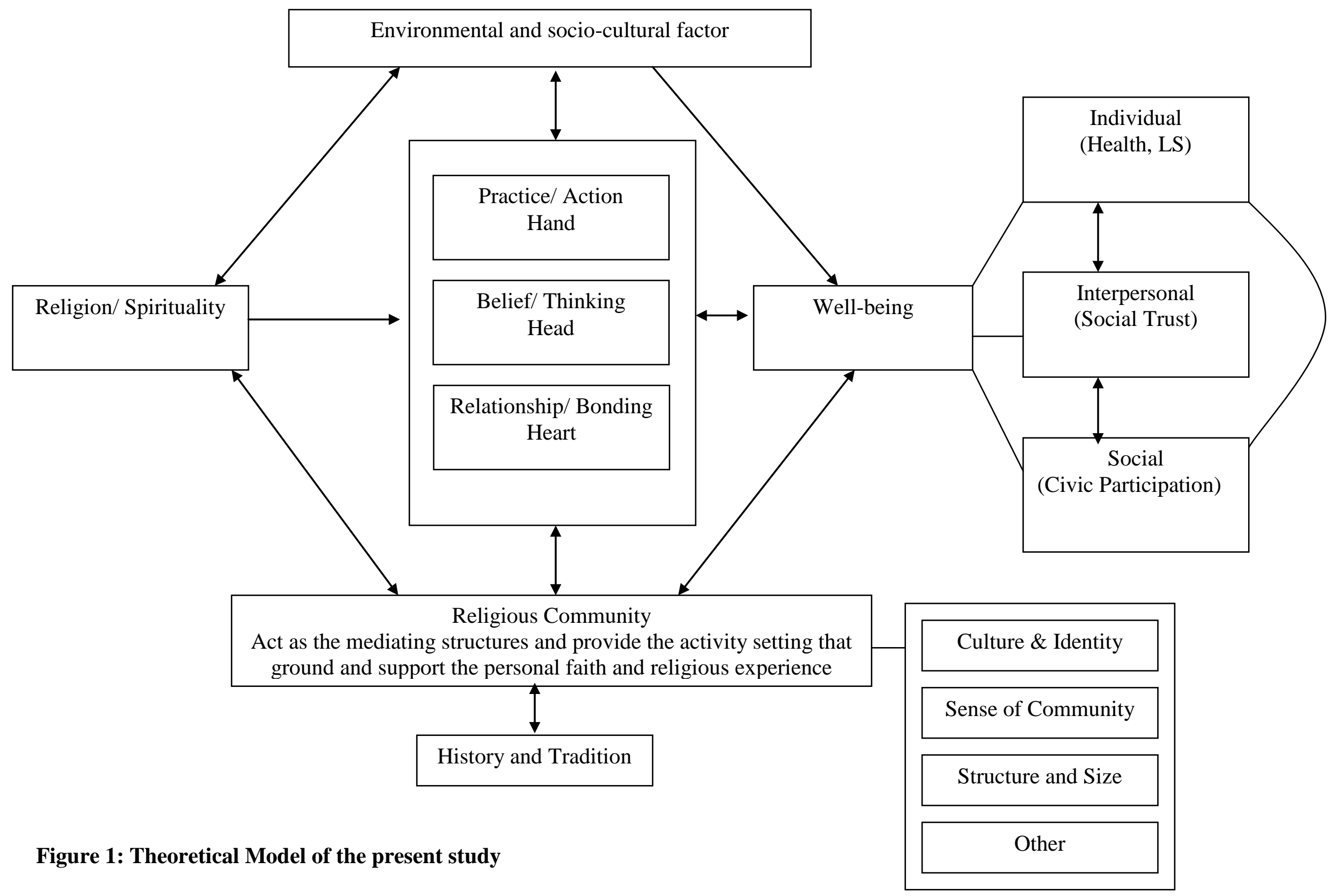




\section{Chapter 5}

\section{Religion and Well-being}

The impact of religion on human beings and human society has long been documented in social science (Durkheim, 1912/2001; Hall, 1891, cited in Gorsuch, 1988; James, 1902/ 1960; J. B. Pratt, 1924; Weber, 1958). Nevertheless, rigorous empirical research on the impact of religion on personal and societal well-being by psychologists has only occurred in recent decades (Maton, 2001; Paloutzian \& Kirkpatrick, 1995) The positivistic stance of social sciences in the $20^{\text {th }}$ century regarded religion as a nonempirical and antiscientific set of faith-based beliefs and many researchers tended not to be religious and viewed religion is a rare and marginal phenomenon (Baumeister, 2002; Ebaugh, 2002; Gorsuch, 1988; Pargament \& Maton, 2000).

Social scientists are now more aware of the need to reintegrate religion into social science (Ebaugh, 2002). This stems from a number of factors, including the continued role of religion in the people's lives, and the importance of religious systems in strengthening individuals, building civil society, particularly in the globalization process. Some say that taking religious pluralism or diversity seriously is the most important development in academic and international politics in the $21^{\text {st }}$ century (Thomas, 2003, 2004; Wuthnow, 2004a).

The impact of religion on individual and community well-being is not difficult to understand. As Dorr (1984) argues, although spirituality is deeply personal, the manifestation of spirituality for a person is not just bounded on that level; spirituality is something that motivates people. Spirituality not only reflects a person's being but also his or her becoming. Given that humans are social being, the experience of the sacred can be revealed in the personal, interpersonal or even the public sphere. Dorr argues that balanced spirituality has enormous impact on personal, interpersonal, and public life. Dionne and Chen (2001) develop these ideas in their book Sacred places, Civic purpose.

In the following section, empirical research of recent decades concerning relationship between religion and well-being is presented. It should be noted that the well-being, discussed in the present thesis, is multi-dimensional in nature. The possible mechanism of the relationship revealed by empirical endeavour will be delineated. Lastly, 
the negative impact of religion on well-being will be acknowledged and some relevant empirical findings will be presented.

\subsection{Religion and well-being}

\subsubsection{Religion and Individual Well-being}

At the individual level, religion and health has aroused much attention (Ferrell, 2008; Kluger, 2009; Koenig, McCullough, \& Larson, 2000; W. R. Miller \& Thoresen, 2003). Koenig et al., after reviewing more than 1200 research studies concerning religion and health, cautiously concluded that "religious beliefs and practices rooted within established religious traditions were found to be consistently associated with better health and predicted better health over time” (p. 591). They did, however, acknowledge that sometimes the opposite can occur. In their handbook, the physical health examined includes mortality, cancer, heart disease, disability or others, while the mental health under investigation includes depression, suicide, mental illness, drug use or other behaviours. Although the effects vary, the positive effects of religion are impressive.

The links between religion and positive notions of mental health are also strong. In the classic article Correlates of Avowed Happiness, Wilson (1967), under the limited information at that time, concluded that a happy person has certain characteristics (such as being young, healthy, well-educated, well-paid), of which being religious is one. After three decades, the connection between subjective well-being and religion have not changed much (Diener, et al., 1999). Others (Argyle, 2000; D. G. Myers \& Diener, 1995) also report that church members, in general, are happier than others and are less likely to engage in unhealthy or possibly damaging behaviour (such as drug or alcohol abuse, premarital and extra-marital sex, or breaking the law). Religiously active people are also healthier, live longer, are less fearful of death, and are generally more satisfied with life. Koenig (Koenig, 1998) recognized that nearly 500 studies (478 out of 724 studies) reported statistically significant associations between religion and better mental health. Koenig (2005) also noted that religion is closely related to positive mental health outcomes, including hope, optimism, meaning and purpose, gratefulness and forgiveness.

Besides physical health and mental health, the positive impact of religion on adolescent moral development has been documented (Erikson, 1964; Regnerus, Smith, \& 
Firitsch, 2003). Erikson claimed that religion is both an ideological and social resource for adolescence development. Recent studies also indicate that religion not only protects adolescents against delinquent behaviour, but also enhances a series of health-related outcomes and social behaviours, such as coping, positive self concept, or social competence (P. E. King \& Furrow, 2004). Some developmental scholars argue that the field can no longer overlook the critical role religion may have in adolescent development (Bension, Roehlkepartain, \& Rude, 2003; P. E. King \& Furrow, 2004).

At the other end of human development, studies also indicate religion has significant influence on people’s aging process (Koenig, 1994; Levin, 1994; Moberg, 2001). Although older people may lessen their participation in organized religious activities, other spiritually-related activities - such as prayer and meditation - still have impressive and beneficial effects on physical and mental health. Studies from a life course perspective indicate that the influence of religion may vary across ages. There are fluctuations in the importance of religion to human life, associated with major life events (such as marriage or getting old), and certain cohorts of people. However, religious influences on people at different ages seem to be consistently positive. Peacock and Poloma's (1998) study indicates that closeness to God is a strong and consistent predictor of life satisfaction. Ferraro and Albrecht-Jensen (1991) indicate that religious practice was positively associated with better health, regardless of age. In summary, religious influence on individual well-being is impressive and wide ranging.

\subsubsection{Religion and Interpersonal Well-being}

On the interpersonal level, studies have shown that religion's impact is striking. Family studies demonstrate the importance of religion and the varieties of religion-family linkages (Holden, 2001; Parke, 2001; Snarey \& Dollahite, 2001). Meta-analysis of religion and family literature over the past 20 years, carried out by Mahoney, Pargament, Tarakeshwar, and Swank (2001), indicates that church attendance is a significant protection against divorce, and higher levels of religiosity can enhance greater marital satisfaction. While more women are religious, the effects of religion on men is more impressive (K. T. Sullivan, 2001). Besides marital relationships, some studies also suggest that religion can benefit parent-child relationships or intergenerational relationships. Religious practice not only facilitates mother-child relationships (Pearce \& 
Axinn, 1998), but some qualitative studies seem to suggest that religious practice can also strengthen father-child bonding (V. King, 2003). In addition, positive family religious rituals, passed down from previous generations, are also shown to be positively related to the marriages in the next generation (Fiese \& Tomcho, 2001).

Homophily $^{2}$ studies also confirm that religious homophily exists in marriage, friendship and confiding relationships, but not in less close relationships, although the degree is not as strong as race and ethnicity (McPherson, et al., 2001). Religion still plays a significant role in building ties (especially close ties) between people.

Studies of altruism also show that religion enhances prosocial behaviour (Saroglou, 2006). Priming studies in social psychology further affirm that religion’s influence on sociability is unconscious, if not automatic (Pichon, Boccato, \& Saroglou, 2007; Shariff \& Norenzayan, 2007). Pichon et al. argue that religious priming may invoke religious ideals and norms, and Shariff and Norenzayan suggest that it may be activation of the felt experience that 'God is watching you'. The close link between religion and prosocial behaviour and the influence of religion on altruistic behaviour seems to be supported.

Furthermore, religion's impact on the interpersonal dimension does not appear to be limited to an individual's immediate circle. Moreover, religion can help build trust. The classic interpersonal trust literature has shown that people with any religion tend to be more trusting than atheists (Rotter, 1967). Erikson (1968) also argue that religious belief is a critical element nurturing basic trust. Delhey and Newton (2003), in a cross national comparative analysis, found that the Protestant tradition had a significant positive effect on trust. Welch et al., (2004) argue that strong bonding within the church can nurture mutual trust, later extending to beyond the church.

\subsubsection{Religion and Collective Well-being}

In the collective domain, the role of religion in forming civil society in the US is recognized (Putnam, 2000; Tocqueville, 2000; Wuthnow, 1999). Civic participation is a key element in the formation of civil society, and the beneficial effect of religion on voluntary work and civic behaviour is a focus of scholars interested in the relationship

\footnotetext{
${ }^{2}$ Homophily is "the principle that a contact between similar people occurs at a higher rate than among dissimilar people.” (McPherson, Smith-Lovin, \& Cook, 2001, p. 416)
} 
between religion and collective well-being. In his award winning book, Acts of Compassion, Robert Wuthnow (1991) concludes that faith has a clear and definite role in facilitating charity behaviour, especially for those involved in some kind of religious organization. Religious people tend to highly value helping people in need, and frequent churchgoers engage in more volunteer activities than non-members. However, Wuthnow acknowledged that what is regarded needs, and when and how to meet those needs, are strongly affected by the religious context. Religious conservatives are sharply distinguished from religious liberals in their underlying values of promoting charitable activity, even though their effect on actual helping behaviour is not vastly different.

After carefully examining changes in religion's impact on civic engagement in the USA since the 1960s, Wuthnow (1999) found that there are close relationships between religious involvement and civic engagement, particularly in relation to mainline Protestants. The religiously active people are more likely to be members of voluntary organizations, and participation in religious settings enhances civic skills which can be transferred to other settings. Putnam (2000), in his Bowling Alone, similarly argues that religion is beneficial to civic life as it directly provides social support to members and social services to the wider community, and also indirectly nurtures civic skills and moral values that motivate helping behaviours and improves the community. Wuthnow and Putnam both worry that increasingly popular evangelical Protestants participate more within the congregation rather than in the broader society, thereby limiting their "salutary effects on America's stock of social capital” (Putnam, 2000, p. 79). However, these scholars do not deny religion's capacity to mobilize civic engagement. Wuthnow (1999) asserts that whether religion has impact, beyond the parish to the broader society, depends on whether the religion can create innovative partnerships with the other bodies in society.

Although religion is notoriously associated with conflicts or even violence between groups of people or nations with different religious faith, the contributory role of religion in bringing peace has also been documented (Ellens, 2004; Silberman, Higgins, \& Dweck, 2005). Thomas (2004) argues that faith-based NGOs may help build mutual trust and humanitarian work from the grassroots. Helminiak (2008) suggests that spirituality, rather than the traditional religion (which focuses more on institutional dogmatism), develops the spirit of humanity that our global world requires. 


\subsubsection{Religion and the building of community}

Religion not only generates a civic engagement of individual congregants, but also provides a context that helps build a community. Durkheim's (1912/2001) classic The elementary forms of religious life carefully outlined how religion is crucial to the formation of society. Religion fosters shared beliefs and practices that are required for a society. By creating a common symbol of their own among members, religion also helps build a common social identity. As a result, religion can powerfully link the individual to society. Durkheim poignantly argued that "if religion generated everything that is essential in society, this is because the idea of society is the soul of religion" (p. 314).

Sarason (1974), in his Psychological Sense of Community, also argues that to understand community, we cannot ignore religious influence because of its strong interrelation with other factors. Recent empirical research also demonstrates that religiosity is significantly associated with people's connectivity in a church context, including a psychological sense of community, perception of social support and identification as a church member (Obst \& Tham, 2009).

Greeenberg (2000) posited that religion has the potential to revitalize political and community life by connecting people. Religious institutions not only can provide political information and participatory opportunities, but also can act as "intermediaries between the individual and the state” (p. 378). Religious institutions often engage in building communities, such as providing shared space for community groups, providing services for the needy and activities for members. Greenberg acknowledges that not religious institutions reach this ideal, because of various constraints (such as demographics, political, or normative), but the promise of a religious revitalization of politics and community is still anticipated. Smidt (2003) similarly noted the close link between religion and social capital. Congregational life is continuously an important component of association life in the USA. Religious beliefs shape the lives of members and religious behaviour "contribute[s] to the social capital formation in that volunteering, charitable contributions, and other distinct acts of mercy can.... help to provide a "safety net" for members of society who are "at risk" (p. 2).

From the above review, there is strong research evidence indicating the beneficial and enhancing effects of religion on well-being, at the individual, interpersonal, or 
collective levels. Moreover, religion also helps people build closer religious contexts to maintain and sustain social relationships and religious faith.

\subsection{The mechanism underlying religion and well-being}

As well as understanding the beneficial effects of religion on the various dimensions of well-being, social scientists also explore the mechanism involved in religion enhancing well-being. One possible drawback of research, however, is the reduction of religion to other non-religious processes, whether psychological, sociological or physiological (Dittes, 1969; Funder, 2002). Therefore, the irreducible account of religion (discussed earlier) is important (Eliade, 1961/ 1999). This study accords with what some scholars have argued (Levin, 1994; Pargament, 2002b); that is, explaining the underlying mechanism does not necessarily mean to "explain the religion away”. Instead, explanation can be used to reveal the underlying pathways through which religion can influence well-being in multiple ways, both religious and non-religious.

\subsubsection{Religion and individual well-being}

Regarding religion and health, Seeman, Dubin and Seeman’s (2003) review of studoes related to biological processes indicates that "spirituality is linked to healthrelated physiological processes - including cardiovascular, neuroendocrine, and immune function” (p. 53). They found that religious influence on health is particularly pronounced in studies of meditation, although they acknowledge that more evidence needed.

Apart from biological pathways, psychosocial pathways between religion and health have received greater attention. However, the mechanisms underlying religion and health are still far from clear. Nevertheless, some promising areas have been proposed. George, Ellison and Larson (2002) identify four major categories of mediators: health practice, social support, psychosocial resources, and sense of coherence or meaning. According to George et al., religious people tend to have healthy life habits and take care of their own bodies. They also tend to have stronger social ties and support with those sharing a similar worldview. Religious participation may strengthen psychosocial resources, such as self-esteem, self efficacy and mastery. In addition, religion offers people meaning and purpose to make sense of their lives, which means they can develop a sense of coherence. Like George et al. (2002), Levin (Ellison \& Levin, 1998; Levin, 
1994) also propose five mediating links between religion and health: healthy behaviour and lifestyle, social support provided within religious communities, positive emotions drawn from the praise and worship, health promoting religious beliefs, religious faith that leads to hope and optimism. He also cautiously suggests that mystical experiences and paranormal divine interventions may also be potential links.

Regarding religion and mental health, Koenig (2005) summarizes ten ways that religion can help improve mental health. They include promoting a positive worldview, helping make sense of difficult situations, giving purpose and meaning, discouraging maladaptive coping, enhancing social support, promoting other directedness, helping release the need for control, providing and encouraging forgiveness, encouraging thankfulness, and providing hope.

Besides explaining religious influence in terms of the biological and psychosocial processes, Pargament (2002b; Pargament, Magyar-Russell, \& Murray-Swank, 2005) persistently argue that religion is a unique process that cannot be easily reduced to other processes. In their paper, Pargament, Magyar-Russell et al. (2005) maintain that religion is a unique form of motivation, a unique source of significance, a unique contributor to mortality and health, and a unique form of coping. As a singular form of motivation, religion is not only uniquely tied to the sacred, but also supersedes all others kinds of strivings (Emmons, 1999). This singular form of motivation is not only associated with higher satisfaction with life and marital relationships, but also with lower level of depression and less conflict related to goals. Moreover, research on sanctification (Pargament \& Mahoney, 2002, 2005) indicates that perceiving something as sacred has a great impact on people in several ways: people tend to invest more in "the pursuit and care of those thing they hold sacred”, “preserve and protect sacred aspects of their lives”, and "derive greater satisfaction and well-being from the pursuit and experience of the sacred” (Pargament, Magyar, \& Benore, 2005, p. 60).

These claims are supported in a series of studies, including on marriage relationships, sexual relationships, and environmental behaviour. Pargament, MagyarRussell et al. (2005) regard religion as a unique contributor to mortality and health and discovered, from recent studies using more sophisticated research, that although some associations between religiosity and health may be reduced to basic psychosocial processes, there appears to be something left, something unique about religion after other 
explanations are examined. Pargament, Magyar-Russell et al. (2005) also found that religious coping - including the sacred in the coping with and appraisal of life - still makes a unique contribution after the non-religious coping method. They argue that the language of religion is associated with the limits of human power, particularly when life is out of control and becoming absurd.

\subsubsection{Religion and interpersonal and collective well-being}

Accounting for the relationship between religion and collective well-being is a more difficult task. Current research is still sparse, let alone conclusive. Some attempts are summarized below.

King and Furrow (P. E. King \& Furrow, 2004) apply the social capital theory to understanding the contributory effects of religion on adolescent moral behaviour. They found that religion facilitates moral outcomes by enhancing three aspects of social capital resources: structural interaction with significant others, trusting relation, and the cognitive dimension. In their words, "the influences of religious importance and participation on moral outcomes are mediated though trusting interaction with adults, friends, and parents who share similar views of the world” (p. 709).

Wald, Silverman and Fridy (2005) draw on three components of social movement theory (culture, resources and political opportunity) to understand why religion can facilitate the political engagement of its members. Firstly, religion is able to tell us who we are and what we ought to do accordingly. By using the "language of framing", religious groups interpret grievances differently to provide justification and motives to the following actions. Secondly, besides offering a culture and identity that is needed for social action, religion is relatively rich in resources, especially in five aspects: culture, leadership, material resources, communication networks, and space. Lastly, but equally important, even though the extent is still largely limited by the wider political environment, religion can extend the opportunity of its members to participate in political life. Greenberg (2000) also supports the idea that religious institutions, as intermediaries between the individual and the state, can serve "as sources of political information, opportunities, resources, and incentives to engage the political process”(p. 378).

As well as accounting for civic life from the sociological theories and perspective, some researchers also try to understand how religion benefits civic participation. 
Nevertheless, research on the underlying mechanisms is still vague, if not ambivalent. For example, Park and Smith (2000) report the significant effect of participation in church activities on community volunteering; however, Loveland, Sikkink, Myers, and Radcliff (2005) did not find such a relationship. Instead, they purport that private religious practice (private prayer) relates to secular civic involvement, even though they realize the effect of individual prayer on civic participation is further enhanced by belonging to religious groups and the content of prayer is also important. Lam’s (2002) study indicates that four dimensions of religiosity (participatory, devotional, affiliative, and theological) have powerful, but distinctive, impacts on secular voluntary association in the US context. Schwadel (2005) concludes that congregational context matters. For example, theologically conservative congregations not only limit members' involving in civic area, but also reduce their connection with other members of the community, thereby decreasing the bridging form of social capital.

These findings show that religious impact on civic life may be mediated variously: both through religious and non-religious means as well as on both the individual and societal level. For example, religion influences civic behaviour not only through changing personal behaviour (like religious practices), but also by providing people with a religious context and a network with resources, which enable them to develop various kinds of civic skills.

In summary, the above review provides us with an intricate picture of the complex relationship between religion and well-being. Religion not only affects well-being on different levels, whether individual or collective, but also influences various kinds of well-being in multiple ways, including through the bio-psycho-social pathways and the the unique spiritual process.

\subsection{Religion and negative well-being}

Obviously, religious impacts are not necessarily positive. As many authors argue, religion is a double edged sword (e.g., Pargament, 2002a; Silberman, et al., 2005). While religion can benefit believers, it can also bring distress and conflict in relationships with others and society.

Allport and Ross’s (1967) classic study indicates that people with an extrinsic religious orientation tend to have more prejudice than those with intrinsic religious 
orientation. Later, Batson, Schoenrade, and Ventis (1993) proposed the 'quest dimension' is correlated with tolerance and compassion, but argued that intrinsic religious orientation may be related to intolerance in some circumstance. As well as religious orientation, Hunsberger (1995) added that people with fundamentalist beliefs and rightwing authoritarianism tend to be more prejudiced. A recent article (Hunsberger \& Jackson, 2005), summarized relevant studies from 1990 to 2003, and delineated clearly how various types of religious orientation influence the type of intolerance and forms of prejudice. To put it simply, people with a fundamentalist orientation tend to be intolerant to various targets of prejudice (like racial/ethnic, gay/lesbian persons, women, or religious out groups), while the people with quest orientation tend to be more tolerant. The effect of other groups is ambivalent. For example, people with intrinsic orientation are not related to racism, but they may show prejudice towards gay/ lesbian people, because that kind of intolerance is not proscribed. This line of argument accords with the work showing the close relation between religion and dogmatism (Saroglou, 2002c), finally leading to increased prejudice (Altemeyer \& Hunsberger, 1992). In addition, Ellens (2004), in his four volume edited series, The Destructive power of religion: Violence in Judaism, Christianity, and Islam, maintains that the apocalyptic worldview held by some religious fundamentalists - "the cosmic contest between the transcendental good and evil” (p. 4) - creates violence and many psychosocial and political problems in societies. Ellens understands that fundamentalists may be serious and sincere about their faith, but he found simplified dualism between good and evil, in addition to the actionoriented nature of Western religion, results in a propensity for violence rather than peace.

Besides prejudice, intolerance or even violence, Bottoms, Shaver, Goodman, and Qin (1995) highlight the fact that child abuse or neglect occurs within the parish or family, on religious grounds. Certainly, losing freedom of thought, facing religious or spiritual struggle, or other religious prohibition is not uncommon to some religious people (Argyle, 2000; P. C. Hill \& Pargament, 2003). As Pargament, Magyar-Russell et al. (2005) argue, not only is religion a unique benefit to human well-being, but it is also a unique source of distress. For example, negative religious coping - such as feeling abandoned by God or perceiving a situation as a punishment by God - was shown to be related to mortality (Pargament, Magyar, et al., 2005). Events perceived to be in violation of the sacred will also have enormous, if not disastrous, effects on the well-being of people (Pargament, 
Magyar, et al., 2005). Different faiths between partners or parents and children may also lead to conflict. Therefore, religion can bring positive or negative impacts depending on the nature of the religion.

\subsection{Summary and Hypotheses}

In summary, the present chapter demonstrates that the religious impact on people is complex. Not only can the religious impact be positive or negative, but its impact can also span different levels, including individual, interpersonal and social domains. In addition, religion can provide a platform for members to build a sustainable network and community in which collective action can be developed and long term change can result. In this study, general health, life satisfaction, social trust, and civic participation are used as variables because the relationship between religion, health and social capital is emerging in psychology.

There are studies showing that members of strict churches have better health than the members of other mainline churches (Argyle, 2000), and it is expected in the present study that churches with strict or moderate religious beliefs will have better outcomes than the liberal congregations. Regarding civic participation, based on Wuthnow (1999)'s study, the more liberal the churches the more involved they are in the society. Regarding social trust in the interpersonal domain, the effect is more difficult to forecast because the conservative congregation may create a boundary between the religious community and the outside world, but the strong social bonding within the "strict” churches may nurture their faith and trust in human nature (Welch, et al., 2004). Thus, it is expected that the level of social trust in conservative and liberal congregations may be roughly the same. More specifically, the following hypotheses have been proposed:

1. Religious groups will have a higher level of spirituality, manifested in religious beliefs, experiences and religious practices, than non-religious groups.

2. Religious groups will have a greater sense of community towards their immediate settings, compared to non-religious groups.

3. Religious groups will have a higher level of generic health and life satisfaction than the non-religious groups.

4. There will be positive relationships between life satisfaction and spirituality, whether regarding religious beliefs, experiences and religious practices. 
5. There will be positive relationships between sense of community and spirituality, whether regarding religious beliefs, experiences and religious practices.

6. There will be positive relationships between sense of community and life satisfaction.

7. There will be positive relationships between sense of community and social trust.

8. The components of spirituality will differentially contribute to the prediction of respondents’ life satisfaction, social trust and civic participation.

9. Age, gender, education and income will be significant predictors of life satisfaction. 


\section{Chapter 6}

\section{The social historical context of the study}

Before we move on to the methodology section, it is necessary to contextualize the present study so that a richer and thicker description of the studied phenomenon can be obtained. In the following sections, information about Hong Kong in general, Protestant Christianity in Hong Kong, and the four studied churches will be delineated in sequence. Certainly, the various realms are not isolated from each other, so the interaction between them will be elaborated as well.

\subsection{Hong Kong in general}

From the time it became a British colony, Hong Kong underwent a unique historical development, developing into an energetic and dynamic city, and earning a reputation as one of Asia’s “four dragons” (Estes, 2005; Tse, 2002). Although it is believed that the history of Hong Kong can be traced back to Han and Chin Dynasties, it is fair to say that Hong Kong gained its increasingly important role and global attention starting from 1842, after the signing of the Nanjing Treaty by the Chinese government. Hong Kong became a thriving port open to the western world. Before that, Hong Kong was subordinate to the coastal provinces in China. Accounts of Hong Kong can be drawn from various perspectives, respectively the colonial government (Endacott, 1964), western missionaries (Eitel \& Lethbridge, 1895/ 1983) and some local scholars (Faure, 2003; Fok, 1990; Lo, 1963; Lui, 1997; C. T. Smith, 1995; G. Wang, 1997). What is common is that Hong Kong is regarded as playing a unique and significant role in the modernization of China and in the exchange of Chinese and western culture.

Scholars find it difficult to tell the story of Hong Kong because Hong Kong is basically a society based on migration; local identity only emerged in the 1970s but was still in flux in the 1980s (Lui, 1997). Because of its unique socio-political history, some writers have described Hong Kong as a borrowed place within a borrowed time (Hughes, 1976), indicating the significance of the socio-political and historical dimensions in the 
development of Hong Kong. Thus, this chapter will focus on the historical account of Hong Kong and the socio-political development of Hong Kong.

\subsubsection{From 1842 to pre-war}

The earliest accounts and records of dwellers in Hong Kong area date from the Han Dynasty (Lo, 1963). At that time, Hong Kong was just a small and underdeveloped coastal port in the south of China. It received some attention, but soon its importance declined compared to neighbouring ports, because of pirates surrounding the area.

The British Empire established a leading in the world and further expanded east. To strengthen its colonial empire, it built operational bases around the world to support trade. Hong Kong, on the lee shore and close to China, soon became the target of the British Empire in eastern colonial expansion. After the Opium war in 1842, Hong Kong was ceded $^{3}$ to Britain and another five Chinese ports were opened to the western world. Since then, Hong Kong not only opened Chinese society to western culture, but also became a colonial society and a stepping stone for westerners waiting to enter the Chinese market. Scholars have argued (Kwong, 2000; Ng Lun, 1989; G. Wang, 1997) that Hong Kong served as an important “gateway between the West and China”. Economically, because of its unique geographical position and favorable environment (such as relative political stability, law and order, free trade and laissez-faire policies), Hong Kong soon developed as the entrepot port and became a key player in China's external trade (Ng Lun, 1989). Culturally, Hong Kong became the centre of cultural interchange between China and the west (Fok, 1990; Lo, 1963). Fok argues that Hong Kong educated Chinese, because of their bicultural perspective, greatly helped Chinese modernization and openness towards Western learning at the turn of the twentieth century.

From 1842 Hong Kong was under British colonial government rule, but the territory still had a very close relationship and frequent exchanges with mainland China until 1949, regarding economic and social development. When disasters or rebellions occurred in China, refugees would flee to Hong Kong to stay for a short period of time before returning home when the situation became calmer (Tse, 2002). Therefore, Hong

\footnotetext{
${ }^{3}$ While the Hong Kong Island and Kowloon Peninsula was ceded to Britain in 1842 and 1860 respectively, the New Territories of Hong Kong was leased to Britain in 1898 for 99 years, meaning it should be returned to China in 1997. Although Chinese government had opportunity to claim back the sovereignty of Hong Kong after the World War II, it did not until the 1980s when the Chinese Communist government opened the door of China again. See Tse (2002) for more detail.
} 
Kong was often regarded as a migration society in which people had no unique and clear sense of identity. The colonial government initiated no long term planning and development of the society because of the high mobility of the population (Tse, 2002). Nevertheless, after 1949, the Communist Party took control of mainland China, and a closed door policy was adopted. As a result, Hong Kong and China became alienated from each other and pursued their separate development.

\subsubsection{From post-war to the 1980s}

Post-war Hong Kong was characterized by tremendous population increases, economic growth and extension of social services (Ng Lun, 1989; Tse, 2002). The civil war in China in the 1950s brought a large number of refugees to Hong Kong. Coupled with natural increase, the population in Hong Kong increased from 1.8 million in 1947, to 3 million in 1960, and to 5 million in the 1980s (Ng Lun, 1989). The growing population brought Hong Kong increased levels of labour and capital, and helped develop the economy, but the territory also developed social issues that the government had to address. Housing was a primary concern. A severe fire in Shek Kip Mei in 1953 forced the colonial government to start providing public housing. The role of government in social welfare and social service developed from the mid-1960s, although it was the voluntary and religious agencies who took the initiative and an active role in the betterment of society in the 1950s -1970s (Ng Lun, 1989; Webb, 1977). In 1971 and 1978, free education was introduced at primary and junior secondary level, resulting in educational development in Hong Kong. All these community building measures, coupled with economic development, slowly generated a unique kind of Hong Kong identity, characterized by the emphasis on freedom, economic success and individual effort (T. W. P. Wong, 2003). Baker (1983) also characterised the Hong Kong 'Man’ as "go-getting and highly competitive, tough for survival, quick-thinking and flexible. He wears western clothes, speaks English or expects his children to do so, drinks western alcohol, has sophisticated tastes in cars and household gadgetry, and expects life to provide constant stream of excitement and new openings. But he is not British or western (merely westernized). At the same time he is not Chinese in the same way that the citizens of the People's Republic of China are Chinese.” (p. 478). Therefore, despite being a Chinese society, Hong Kong developed a unique kind of ethos different from mainland 
China (Lau \& Kuan, 1988). Despite the socio-political nature of Hong Kong society, the 'Hong Kong Man’, ironically, had no strong interest in political matters. Instead, an amoral individualism was deeply embedded in the Hong Kong ethos (Lui, 1997; T. W. P. Wong, 2003), resulting from the transient nature of society and colonial governance which did not provide integrative symbols for human striving (T. W. P. Wong, 2003).

\subsubsection{The return to Chinese sovereignty in 1997}

In December 1984, the Sino-British Joint Declaration was signed, indicating that British sovereignty over Hong Kong would be terminated in 1997. Although the colonial government initiated political reforms in the early 1980s and introduced direct elections to District Boards in 1982 and the Legislative Council in 1991, the people of Hong Kong had no say in determining their own future in the negotiations between the Chinese and British government (Kwong, 2000). As the wealthy tried to emigrate overseas, especially after the Tiananmen Square massacre in Beijing in 1989, others who were not able to leave remained indifferent to the broader social context.

The return to Chinese sovereignty in 1997, however, was surprisingly smooth and calm, and the "one country-two system” principle was enacted. Instead of worrying about interference from the central government of mainland China, the Hong Kong people’s trust of the central government was high (W. M. Lam, 2007). The more pressing problems in Hong Kong by the end of 1997 were domestic issues, such as the Asian economic crisis and the bird flu (Kwong, 2000). In 2003, a series of social and political events, as well as the poor governance by the Hong Kong government, brought forward an outburst of dissatisfaction from Hong Kong citizens, which resulted in the $1^{\text {st }}$ July rally with 500,000 protestors, symbolizing the emergence of people power and civil society in Hong Kong (J. M. Chan, 2005).

Nevertheless, Lam and Perry (2000) and Lam and Tong (2007) observe that, while civil society in Hong Kong is vibrant, because of the underdevelopment of the political structure, the influence of civil society on government policy is very limited. Ma (2007) also argues that the post-1997 crisis in Hong Kong was due to incongruities between the state and society's demands, and the fact that the government did not handle the rapidly changing relationship between the state and civil society well. Estes (2005) also notes out that, although Hong Kong has developed economically, being a most 
advancing society in global terms, the development of Hong Kong is still far from satisfactory because, in reality, not all people and sectors share the fruits of economic development. Instead, the discrepancy between the rich and the poor is severe. That is why Estes (2005) advocated a more balanced approach between the economic and social development in Hong Kong.

\subsection{Protestant Christianity in Hong Kong}

From the history of Hong Kong, we find the development of Hong Kong society not only relates to the colonial expansion of Great Britain, but also closely intertwines with western missionaries and the development of Christianity in China. Christians (including Protestant and Catholics) in Hong Kong total about 10\% of the population, and over $60 \%$ of social charity works (N. W. Kwok, 1997; Kwong, 2000; Ying, 2004) and around 50\% of educational services (Central Policy Unit, 2004; N. W. Kwok, 1997; Kwong, 2000) are provided by Christian churches or organizations, reflecting the disproportional role of Christianity in Hong Kong society and development. In the following section, how Protestant Christianity was established and developed in Hong Kong and its relationship to the society will be discussed before we turn to the four studied churches.

\subsubsection{From 1842 to pre-war}

Before it was ceded to Great Britain in 1842, there were already missionaries arriving in Hong Kong as well as China. The first missionary was Robert Morrison, from the London Missionary Society, who landed in Macau in 1807. In 1841, one year before the signing of treaties, eight western missionaries visited Hong Kong (Liu, 1941). Since then, several Protestant denominations established churches in Hong Kong, and regarded this small place as a stepping stone and base of operations towards the greater harvest in mainland China. Before the twentieth century, there were already seven established denominations in Hong Kong: the London Missionary Society, Congregation church, Southern Baptist Convention, The Anglican Church, Wesleyan Methodist Missionary Society, Basel German Evangelical Missionary Society, and the Barmen Missionary Society (Liu, 1941; Ying, 2004, p. 12). All these denominations have a very long history in (and close affiliation with), the western world. 
During this period of time, the critical issue regarding the church in Hong Kong was the transition of governance from western missionaries to local Chinese Christians. Although the western missionaries were that rare group of westerners in Hong Kong who were more willing to approach the Chinese, the development of Protestant Christianity in Hong Kong was still limited because of the high mobility of people in Hong Kong (Ying, 2004). Christian churches grew and declined as the migrants moved in and out of the society. Ying (2004) notes that if Hong Kong was a borrowed place within a borrowed time, the church in Hong Kong was also a "borrowed church" (p. 73). Ying further argues that, although Protestant churches in Hong Kong markedly developed from the late nineteenth to the early twentieth century, strictly speaking there were no Hong Kong churches before 1949, because churches in Hong Kong were considered to be stations in the regional strategic plan of western missionary work in south China. A primary concern for Hong Kong’s particular situation was lacking.

\subsubsection{From post-war to the 1980s}

Another important watershed in the history of Protestant Christianity in Hong Kong occurred in 1949, when the Communist Party took full control of mainland China (Ying, 2004). The political change in mainland China forced western missionaries to evacuate to Hong Kong, a bridgehead or last stop where they could focus on missionary work. The resources these missionary organizations brought to Hong Kong were a timely help to relieve social problems caused by the massive influx of immigrants from mainland China. In this period, besides the evangelical ministry, Protestant churches in Hong Kong also played a very important role in social development, by providing education, medical services, and social welfare through their affiliated schools or colleges, hospitals, and social welfare agencies (Webb, 1977; Ying, 2004).

As the closed door policy was adopted in China, the Protestant churches in Hong Kong started to develop a local identity. During this period of time, the number of believers in Hong Kong Protestant Christianity grew rapidly from less than ten thousand before 1949 to more than one hundred thousand $(112,200)$ in the early 1960s (Noren, 1963; Ying, 2004, p. 177), and then 270,000 in 1989 (Wu \& Fok, 2006). The numbers of congregations also increased from 188 to 344 between 1955 and 1962 (Noren, 1963; Ying, 2004) and 634 in 1980 (Wu \& Fok, 2006). It should also be noted that in the 1950s, 
the number of denominations was estimated to be over 50 , far beyond the seven established denominations before the turn of the twentieth century (Ying, 2004).

Alongside the increase in the number of believers, the spiritual orientation of the Protestant church in Hong Kong also experienced a major shift, from a moderate stance to a more conservative and fundamentalist orientation (Ying, 2004). This shift was apparent in two ways. Firstly, because of their conservative doctrinal outlook, apart from providing relief work, the majority of Hong Kong Protestant churches (especially the evangelical churches) evaded social-political involvement in 1950-60s (W. L. Kwok, 2002). This doubtlessly matched with the social atmosphere of that time in which people tended to eschew worldly affairs (Ying, 2004, p. 175). Another reason is that the evangelical churches had not articulated well or resolved the dilemma concerning the role of social concern in a dominant fundamentalist religious faith tradition, which emphasized the primacy of preaching the gospel. That explains why conservative churches in the 1960s were criticised as being apathetic towards social problems, which gave rise to a number of para-church organizations ${ }^{4}$ which attended more closely to the needs of the younger generation, grassroots people, and social issues in the 1970s (W. L. Kwok, 2002). Secondly, the established mainline Protestant churches were still active, if not expanding, and taking up the responsibility for providing service in education, medical, and social service in the 1960s, but expensive social welfare later became a burden to the church. As the support from the foreign missionary societies diminished in the mid-1960s, local churches had to rely on government subsidies and sponsors $(\mathrm{N}$. W. Kwok, 1997; Y. M. Wong, 2001). The consequence of this kind of partnership is that Protestant churches were no longer able to emphasize their evangelical ideal as the ultimate goal of their work (Y. M. Wong, 2001). Another major implication of this close partnership with government is a reduction in the church’s independence and prophetic voice in social political issues in the 1980s (N. W. Kwok, 1997).

\footnotetext{
${ }^{4}$ According to Kwok (2002), several para-church organizations have been set up to respond actively to societal needs. For example, the Breakthrough organization and the Fellowship of Evangelical Students were initiated to deal with the needs of the younger generation, while the Industrial Evangelical Fellowship was concerned with labourers in industry.
} 


\subsubsection{The return to Chinese sovereignty in 1997}

Stepping into the 1980s, the demand for greater involvement of Protestants in society, if not politics, was becoming more critical due to political transition and, like many citizens, Christians in Hong Kong became more concerned about the prospects of Hong Kong. For example, in April 1984, a few months before the signing of the SinoBritish Joint Declaration, Christian leaders published a document ${ }^{5}$ that expressed their commitment towards society, and outlined the role and responsibilities of churches (W. L. Kwok, 2001). Moreover, an important minority of Protestants advocated an egalitarian and democratic society (Luk, 1989). According to Chan (1999), they adopted a critical opposition stance toward the government. Nevertheless, it should also be noted that there were also Protestant leaders, like the Anglican bishop, who were more aligned with political authority, whether British or that of Beijing (N. W. Kwok, 1997; Luk, 1989). As Kwok (1997) explains, the churches in Hong Kong were heavily dependent on the government (either colonial or Chinese) for their financial resources to provide social service and charity work, and they lost their prophetic spirit, for fear of losing status and influence. Kwok (1997) notes that Hong Kong churches have been involved in very impressive work in the development of Hong Kong in the 1960 and 1970s, through their partnership with government in providing social service; however, by the 1980s, they had become "victims of their (own) success" (p. 31). Moreover, in the 1980s, they lost their prophetic voice, and spirit for a more human and equitable society.

Approaching 1997, the social involvement of Christians lessened. Like many citizens facing a "crisis of confidence" in the 1980s, there were also people of faith who made decisions about either remaining or retreating. Protestant churches in Hong Kong, however, also attempted to win souls before the "deadline" in 1997. As a result, many evangelical meetings were organized and churches expanded rapidly. From church statistics, the number of believers in Hong Kong Protestant churches grew from 270, 000 to 390,000 between 1989 and 2004 (Wu \& Fok, 2006). The number of congregations also increased from 634 to 1181 between 1980 and 2004 (Wu \& Fok, 2006).

\footnotetext{
${ }^{5}$ The document was called The Conviction of Hong Kong Christians in the Present Social and Political Changes. Although the document was drafted by a small group of the young Christian leaders, it was endorsed by about 3000 Christians and over 200 church leaders and workers in Protestant circles. See Kwok (2001).
} 
After the return to Chinese sovereignty, the church-state relationship also entered a new phase. As some observe (Kwong, 2000, 2001), the influence of Protestant churches in the Hong Kong society declined because of the change of government, which no longer granted the Protestant church special privileges. Kung (2004) observes that tension existed between Beijing and churches in Hong Kong (especially the Catholic church, where Bishop Zen often made high profile criticism of government policy). Nevertheless, others (e.g. Chow, 2001) thought that the decreasing status of the Protestant churches was natural and not negative, particularly as Hong Kong is a global city where pluralism is necessary. Kung also argues that the tension between religion and politics is unavoidable, but not unhealthy. Instead, it indicates explicitly that the "one country, two systems" can be realized despite still undergoing alignment.

From this very brief overview of Protestant Christianity in Hong Kong, we discover the intertwined relationship between the Protestant churches and the development of Hong Kong society. Protestant churches, relying on the special status of Hong Kong as a colonial place, developed and consolidated their missionary work in Chinese society. Their investment and work in the development of Hong Kong society in the 1950s - in times of need - bore fruit and they gained a favourable reputation with citizens. In general, the Protestant churches in Hong Kong adopt a supportive role in relation to government, often in partnership with government. However, the relationship with government, be it partnership or critical opposition (S. H. Chan, 1999), is still very dynamic and evolving as the social and political environment changes.

\subsection{The five settings in the present study}

After reviewing the historical and socio-political context of society in general and of Protestant churches in Hong Kong, the focus now shifts to the churches of concern to the present thesis. The four congregations in the present study happen to represent the several important periods in the development of Protestant Christianity in Hong Kong. While the social concern church is one of the earliest established churches in Hong Kong, the evangelical church was established in the era characterised by the influx of immigrants fleeing civil war in mainland China. While the charismatic church represents Christian effort in the 1970s and was related to the global charismatic movement, the fundamentalist church represents indigenous evangelical zeal and the churches of the 
1990s. More importantly, the four studied churches represent the four major types of Protestant spirituality. According to Chan (2002), the Protestant Christianity in Hong Kong can be mainly divided into three types: evangelical church, mainline church and charismatic church (p. 387). But understanding that there are relatively more congregations in Hong Kong belonging to the evangelical camp (Ying, 2004); this camp includes both the relatively moderate evangelical population and the more fundamentalist evangelical groups. There is no formal statistics regarding the number of congregations for each religious type, and proportionate sampling cannot be obtained. Instead, equal weight sampling method is used in the present study. Moreover, these four types of congregation are also aligned along the fundamentalist-liberalism continuum mentioned in Chapter Four (4.2.4.2). Two religious communities are affiliated to the moderate end (one being evangelical and the other charismatic); another two religious communities represent either end of fundamentalist-liberalism continuum (socially concerned and fundamentalist). A very brief overview of the four churches follows below, while more detailed information about the operation of each church is presented in Chapter 12. For convenience, four associated abbreviations will also be used throughout the thesis: CHC for charismatic church, SCC for social concern church, FDC for fundamentalist church, and EVC for evangelical church.

\subsubsection{Charismatic church - CHC}

CHC is a medium-sized church with a charismatic background. Established in 1969, CHC was founded by an overseas missionary who was called to serve China. After several expansions and relocations, CHC now serves 500 members, cutting across all ages, and is located in a district close to the city. Although the founder of the church is still the pastor in chief, in recent years the leadership in Hong Kong has shifted to his daughter and son-in-law and several newly ordained pastors, while the founder engages in missionary work in mainland China. The majority of congregants are Chinese, but there are also participants from non-Chinese backgrounds. Some of them are just visitors while some are missionaries waiting to be sent to the field. A unique feature of CHC is the focus on the work of the Holy Spirit. Speaking in tongues and spiritual healing are not uncommon practices in $\mathrm{CHC}$, which develops a very special kind of spirituality within 
the Christian tradition. It should be noted that the charismatic tradition is not exclusive to Protestant Christianity, but is also seen in the Catholic tradition.

\subsubsection{Social concern church - SCC}

SCC is a small church that is persistent in its social concern orientation. Despite having a history stretching back over 100 years, SCC has less than 100 members. Nevertheless, SCC has developed an unusual, if not unique, culture and character within the Christian community in Hong Kong. The critical period for SCC was in 1960s, during which a chapel was purchased and built and a visionary pastor joined. Most of the current rituals, practices, and the church spirit of SCC can be traced back to those early years. Nevertheless, SCC, has undergone tremendous change in recent decades. Not only has the turnover of leadership been frequent, but also the conflicts and politics happenings within the church have greatly hindered church development. In the year in which the present study was conducted, a female pastor (in charge of SCC for nearly three years) resigned from the position just few weeks before the data collection phase commenced. After more than four months of recruitment, a male pastor reported for duty. In the four months without a pastor in charge, deacons and lay leaders of the church played an active role in leadership. SCC now belongs to a denomination (now with more than 50 congregations) founded in the very beginning of Hong Kong's development. Like mainline Protestants churches in western countries, SCC and its denomination are actively involved in wider society; however, like its western counterparts, its development is not as rapid as other evangelical and stricter churches.

The SCC is located in an old area (at least in Hong Kong terms), that dates back to the 1950s, and currently being redeveloped. The demography of the SCC congregations is skewed towards both ends - concentrating on adolescents, young adults and elders, while those aged 30-40 years old are fewer in number, if not completely absent. Judging by the clothes people wear, a small number of people in SCC come from poor socioeconomic backgrounds. It is also known that some SCC members belong to the same kinship family, or even extended family. 


\subsubsection{Fundamentalist church - FDC}

FDC is a medium-sized church with a fundamentalist orientation. Established in 1996, FDC now serves about 200 people and is a branch seeding church whose mother church was set up in the $1960 \mathrm{~s}^{6}$. Although it was initially started by about 10 people who left a more established church from an evangelical background, these new flocks now have developed into large groups of people with more than 30 meeting places serving more than 5000 people. They also have an affiliated seminary, kindergartens and some social service centres under their umbrella. The religious outlook of FDC and its sister churches is relatively conservative, if not fundamentalist, because of its roots in the Brethren movement tradition, which emphasizes pietism and the importance of scripture. It is the author's understanding that separate seating for male and females during church activities is still practiced by some of the sister churches of FDC. Nevertheless, it is acknowledged that there is diversity among the 30 meeting places. Leadership is performed in the FDC by an elder, three pastoral workers and with support from some deacons in the church.

The FDC church is located in the centre of Hong Kong, in an old but vigorous district, five minutes walk away from major public transport hubs. FDC occupies a commercial building near construction material companies and a few streets away from night clubs and saunas which provide sex services. FDC members are mainly adults, above 30 years of age. A Saturday service specially designed for teenagers and youth, (12-18 years of age), was started at the beginning of the studied period.

\subsubsection{Evangelical church - EVC}

EVC is a mega-church with evangelical zeal. With more than 50 years of history, EVC now serves more than 5000 congregations and belongs to a denomination of more than 100 congregations serving more than 30,000 believers in Hong Kong. With its good reputation, large size and leading role in the denomination, it is fair to say EVC is one of the key players in the evangelical camp in Hong Kong. To serve more than 5000 members in the congregation, the pastoral team of EVC is huge. According to 2007

\footnotetext{
${ }^{6}$ To be more correct, FDC is a breakaway branch of MFDC (the mother church) and of SFDC, which is also the breakaway branch of MFDC established in the 1980s. Since MFDC is the founding church of all the meeting places later on, including FDC, MFDC will be referred as the mother church throughout the chapters.
} 
statistics, there were 45 pastoral workers helping in pastoral work in the church at that time, and more than 2000 members (42\%) were also involved in serving the church.

EVC is located in a well off district in Hong Kong close to a major public transport interchange, and commercial and residential buildings. Due to its large congregations, EVC now has four gathering places, located in four separate but nearby buildings in the same region within 5 to 10 minutes walking distance. The main hall of EVC is at the exit of a railway station, and therefore the flow of people is huge, especially on Sunday morning. It is also believed that some church participants are people passing by. EVC congregations are very diverse. Although adults dominate the population, there are groups or fellowships for teenagers or children. There are special services for people in Mandarin and English. Moreover, a group of people with physical disabilities also attend services on Saturday.

\subsubsection{The comparison group - Social centre}

Following this brief introduction to the studied churches, it is also necessary to provide a brief understanding to the comparison group in our survey study - the social centre. The social centre is a faith-based organization providing a wide range of secular social services to the general public, including employment training, school support services, a service for the elderly, counselling, family services, and community development. Social service organizations, either religious or non-religious, are normally partners in service provision: the government provides funding and the organization provides services. For all organisations, including religious organizations, providing quality services is the primary goal and the religious atmosphere is not strong, if it exists at all. As a result, it would be more accurate to describe such services as social centres rather than religious organizations. The participants in the present study mainly belong to the continuing education section of the agency, which offers youth and adult interest classes, as well as professional certificate or diploma courses. The questionnaires were distributed mainly in three professional diploma courses and the adult interest class, and participation was voluntary.

Following this overview of the socio-historical context and a brief introduction to the studied settings, the methodology employed by this study, and its rationale, will be discussed. 


\section{Chapter 7}

\section{Methodology}

Whether, how, and why spirituality and religious communities enhance wellbeing are the major research questions of the present study. This investigation will combine quantitative and qualitative methods: a field study including participant observation, semi-structured interviews, and documentary analysis. This mixed method approach is rooted in both philosophical and practical reasons which are explained in detail.

\subsection{The rationale of using Mixed Method}

\subsubsection{The limitation of conventional approach}

In psychology, the positivistic, deductive empirical approach has dominated the field since it separated from philosophical roots. It keeps a distance from the phenomena it aims to study (Flyvbjerg, 2001), and also has limited and narrowed its point of view, if not mission (Kral, 2007; Rappaport, 2005; Trickett, 1996). This is manifested in several aspects. Firstly, conventional scientific investigations have been criticised for their failing to take seriously the subjective experience of those being studied (e.g., Bruner, 1990; J. A. Smith, Harré, \& Langenhove, 1995). Secondly, the overemphasis on quantitative methods and the relative disregard of qualitative methods constrain the ability of psychology to capture the rich contextual information of the phenomena (Banyard \& Miller, 1998; Trickett, 1996), and the core essence of community research and action (Primavera \& Brodsky, 2004). Thirdly, in empirical investigation it is not ensured that researchers’ imposed variables or categories are culturally anchored and valid to the studied phenomena, especially with culturally diverse backgrounds (Maton, 1993). Last, as argued by Flyvbjerg (2001), given that the ultimate goal to generate a rule, scientific investigation is still unable to prepare social scientists to be "experts" in understanding social phenomena because experts require intuitive and holistic understanding, characterized by a flowing and effortless performance, which is context dependent and which cannot be offered by rational rules. 
Nevertheless, the author does not reject the value of quantitative and empirical approaches to social science. As argued by Byrne (2002), any studies of community are too big and the context is changing too fast; so, quantitative measures can be used to contextualize or provide a snapshot of the studied phenomenon. By using complex measurement, we can draw nearer to reality. Therefore, instead of fully embracing or discarding the empirical quantitative approach, we acknowledge and highlight the limitations of the conventional approach, which can be complemented by using multiple research methods. Moreover, to strive for excellence in community research (Rapkin \& Mulvey, 1990), the author uses a mixed method approach, avoiding overemphasis on the conventional approach at the expense of other qualitative approaches; the researcher therefore can gain better understanding of social phenomena. As Axinn and Pearce (2006) argue, mixed method approaches enable researchers to achieve a balanced method and comprehensive empirical documentation counterbalancing the weakness inherent in a specific method, and thereby consolidating the claims of potential mechanisms discovered in the studied phenomena. In the following section, ethnographic and narrative research approaches will be discussed to illustrate how these research approaches can complement the conventional approach to studying human phenomena.

\subsubsection{The contribution of qualitative research approach}

Bruner (1990), in his Act of Meaning, criticized positivistic science that constrains psychology from understanding the meaning making of humans. The cognitive movement of 1950s, in response to behaviourism, tried to go beyond the simple stimulusresponse model of human behaviour and take the human mind into consideration, but the technical information processing model was still based on computation and the simple operating mechanism, rather than fully embracing the diverse meaning of human agency. According to Bruner, to really understand the meaning and the processes involved in creating meaning, it is necessary to study the culture and language in which symbolic meaning is embedded. Likewise, community psychology, since its inception, has been against the ahistorical and acontextual notion of the human in mainstream psychology, which makes it a new branch of psychology that emphasizes and strives for the study of ecology and community context, as well as attention to process (Linney, 2005; Prilleltensky, 1994; Wandersman, Kloos, Linney, \& Shinn, 2005). The common 
complaint about mainstream psychology is the limitations of conventional methodologies, which do not allow psychologists to fully understand the subjective meaning of "person" or the intricacies of interactions between humans and contexts, both of which are the core elements in the discipline. In addition, the emphasis on studying the person in context and the person as an agent, rather than an information processing device, apparently and inevitably leads to a research approach which is holistic, qualitative and interpretive. To attain their goal, two promising approaches are attracting more attention.

Narrative approaches have recently gained attention in the psychological research area (Andrews, 2000; Giles, 2002; M. Murray, 2000, 2003). It is considered especially important to gain a subjective perspective and to understand and explain social and psychological phenomena that span the different levels of analysis (Andrews, Sclater, Rustin, Squire, \& Treacher, 2000; Atkinson, 1998; Mankowski \& Rappaport, 2000; Salzer, 1998). Murray (2003) argues that we live in a storied world and that we interpret the actions of others and ourselves through the stories we exchange. We not only shape the world through the stories we tell, but also are shaped by the narrative around us. Through the storytelling, people organize their experience and make sense of their life as well as answering the question "Who am I?” (Atkinson, 1998; M. Murray, 2000).

Because of the social nature of narrative, experience is organized in a narrative form that can be grasped by others; the narrative embodies the relationship between the narrator and the audience, as well as the broader social context (M. Murray, 2003). Narrative accounts enable the researchers to explore the distinct "psychosocial zone" (Andrews, Sclater, Rustin, Squire, \& Treacher, 2000) of a person, including both the outer socio-cultural realm and inner psychological realm.

Mankowski and Rappaport (2000) used “personal story” and “community narrative” to describe narratives occurring at the personal and communal levels. Whatever nomenclature one uses, it is agreed that by detailed documentation and analysis of the personal story and narrative within the community, the researcher not only understands the meaning making process and the psychological world of persons, but also can realize the social reality and context of a given situation (Andrews, et al., 2000; Atkinson, 1998; Mankowski \& Rappaport, 2000; M. Murray, 2003). Maton (2000) argues that a narrative approach can provide information about the culture of the setting, its impact on members, and the mechanisms by which the different settings exert influence 
over members. Therefore, a narrative approach can capture the reciprocal relationship between individuals and society and the multi-layers of analysis of a phenomenon (Mankowski \& Rappaport, 2000; Rappaport, 1995; Salzer, 1998).

We can draw on personal narrative accounts to understand cultural settings, but to provide a thick description of phenomena, observations in context are necessary (Geertz, 1973). To realize this goal, ethnographic field research is appropriate because it is good at providing information about groups of people interacting with each other, on site, in ways which are constantly changing, complex, and without any fixed form, all of which is less comprehensible to outsiders (Neuman, 2006). As well as providing information about the routines that make up organizational life (Schwartzman, 1993), the ethnographic approach can also enable researchers to understand the cultural meaning collectively shared among members of an organization or a community (P. J. Miller, Hengst, \& Wang, 2003). Neuman (2006) is correct to note that ethnography studies are good at providing detailed description of a context, especially the explicit and tacit cultural knowledge used by members in the context. Kral (2007) argues that, through the interpretive ethnographic approach, both the subjectivity of the individual and the objective cultural world can be revealed. Schwartzman (1993) also has similar ideas, that ethnography not only helps researchers to learn about a culture from the inside out, but also enables them to link between the micro-level, interactional processes and the macrolevel structure. Tedlock (2000) contends that, combined with other methods, ethnographic fieldwork can "produce historically, politically, and personally situated accounts, descriptions, interpretations, and representations of human lives” (p. 455). Combining surveys with ethnographic observation as well as understanding personal narrative accounts can therefore enable a better understanding of social phenomena.

\subsubsection{The importance of mixed methods in the present study}

The use of mixed methods is necessary, if not imperative, for several reasons. The issues involve a complex system, interweaving both individual (spirituality) and social spheres (religious community) in many dimensions. Instead of seeking simple explanations using either quantitative or qualitative traditions, the author concurs with Byrne (2002), and considers quantitative or qualitative inquiry and modelling as part of an array of methods. They need to be compared and understood in parallel with other 
possible, but valid, methods before any definite account of the phenomena can be reached. Therefore, this study uses a more expansive and inclusive paradigm (Greene \& Caracelli, 2003). Multiple methods will be used only if they can shed light on and make sense of the issue.

Moreover, as mentioned earlier, the insider-outsider problem is crucial in the study of religion (McCutcheon, 1999). McCutcheon observes that there are two major ways to study human behaviour, including religious behaviour. One attempts to understand the meaning and motives underlying human behaviour, while the other looks for trends and regularities so that explanations and predictions can be made through interpreting data. The first takes an emic stance, and empathetically understands human experience from the inside. The second one adopts an etic stance to critically examine from the outside. Both are valid approaches to study religion.

McCutcheon (1999) also notes two other theoretical options in the study of religion. The first takes a neutral stance regarding truth and value, but still strives to describe, delineate and articulate the complexity and relations of phenomena in the hope that interesting findings can emerge. The second, aligning with postmodernism, maintains that we are only able to know our own position. According to this perspective, all claims of truth or neutrality are misguided except meaning in relation to the reflexivity of researchers. The scholar needs to reflectively articulate their own point of view, regarding the studied issue, for public examination. As religious faith is personal, it should be respected in an era of diversity (Wuthnow, 2004a). However, manifestation of religious faith is public and social, and should be monitored, particularly realizing that religion can be sweet and bitter (Pargament, 2002a). Therefore, neither etic nor emic perspectives will dominate; both the insider and outsider perspectives are used to understand phenomena, revealing a more complete picture.

Although the empirical approach to the study of religion and well-being is familiar and useful, it is still lacking if we take seriously the issue of the person in context. As discussed in Chapter Three, to really understand the person in context, information about the multiple level of context is needed (i.e., the practices and routines performed by people, the micro-meso system, or even macro system), and the objective and subjective realm of context. Survey data cannot fully reflect the complexity of the issues, and 
therefore complementing the survey with qualitative approaches can provide a fuller understanding of the researched phenomena.

Moreover, besides the survey approach, ethnographic and narrative approaches are suitable to the study of religion and well-being. Mankowski and Rappaport (2000) argue that a narrative approach is particularly suited to describe and analyse spirituallybased communities because religious communities are full of narrative (biblical text, legacy or personal sharing) used to guide and shape the individual and collective lives, and for religious purposes. Collective identity and cohesion are high while the narratives are well developed and communicated very explicitly. Atkinson (1998) also notes that story telling can tell us what is of most value to a person. By studying life stories, we can enter the sacred realm of a person to know what matters most in a life and gain understanding of "human dilemmas, human struggles, and human triumphs, as well as a greater appreciation for how values and beliefs are acquired, shaped, held onto, experienced, and understood” (p. 14).

Weisner (1996) also maintains that ethnography is the most promising method to study human development, including well-being. Weisner contends that any important question in human development cannot be answered with a single method because every setting is particular and methodcentrism not only indicates the imperialistic attitude of specific disciplines, but also leads to bias and distortion. Weisner argues that to really understand the development and well-being of people, cultural place has to be examined in detail, because it is cultural beliefs, practices, meanings, and ecological settings that significantly influence, if not determine, human development. In other words, to understand well-being, multiple methods need to be used.

Lastly, in addition to philosophical reasons, mixed methods are used for practical purposes, including the nature of research questions, as well as the ability to provide a more holistic picture of the phenomena. The survey will provide objective data on health and well-being, and will facilitate modelling with types of community. It cannot, however, provide sufficient information on the dynamics of the religious context. Moreover, the forced choice variables used in the survey cannot capture all the complexity and multidimensional aspects of spirituality and religious community (J. Miller, 1997).

Furthermore, the evolving interaction between the researcher and the researched is also one of the areas that cannot be captured by questionnaires with a preset framework. 
Finally and most importantly, the subjective account and meaning of religious experience and behaviour cannot be revealed without the help of qualitative methods (Banyard \& Miller, 1998). A mixed method approach, however, can prevent the limitations inherent in some forms of enquiry, and can answer questions that cannot be addressed by just one research method (Maton, 1993; Tashakkori \& Teddlie, 1998).

\subsection{The procedure and structure of the present study}

In this thesis, a mixed method will be adopted based on the philosophical and practical reasons discussed. While the quantitative survey provides us with a snapshot of phenomena, qualitative methods, combining both participant observation and interview data, provide contextual information and human subjective information to interpret and inform the quantitative results. Creswell, Clark, Gutmann and Hanson (2003) identified six commonly used designs in mixed methods research design, and the present study uses two of those: sequential explanatory and concurrent triangulation design. A visual model of mixed methods, as discussed by Creswell et al. (2003), and Teddlie and Tashakkori (2009), is used in the present study (see Figure 2), to summarize and clarify procedure. The quantitative survey is the main driver of this study, complemented by qualitative study. This provides richer understanding of phenomena and an explanatory account to triangulate and illuminate significant survey findings in what Teddlie and Tashakkori (2009) term crossover track analysis. Although the quantitative and qualitative study are independent, both sets of data and analyses are used in analysis. In the following chapters, the survey examining the relations and associations between the key variables will be presented first. Next, the qualitative description of the religious setting will follow to situate the study and inform interpretation. Then, qualitative data and survey findings will be integrated and discussed further to provide deeper understanding of phenomena. 


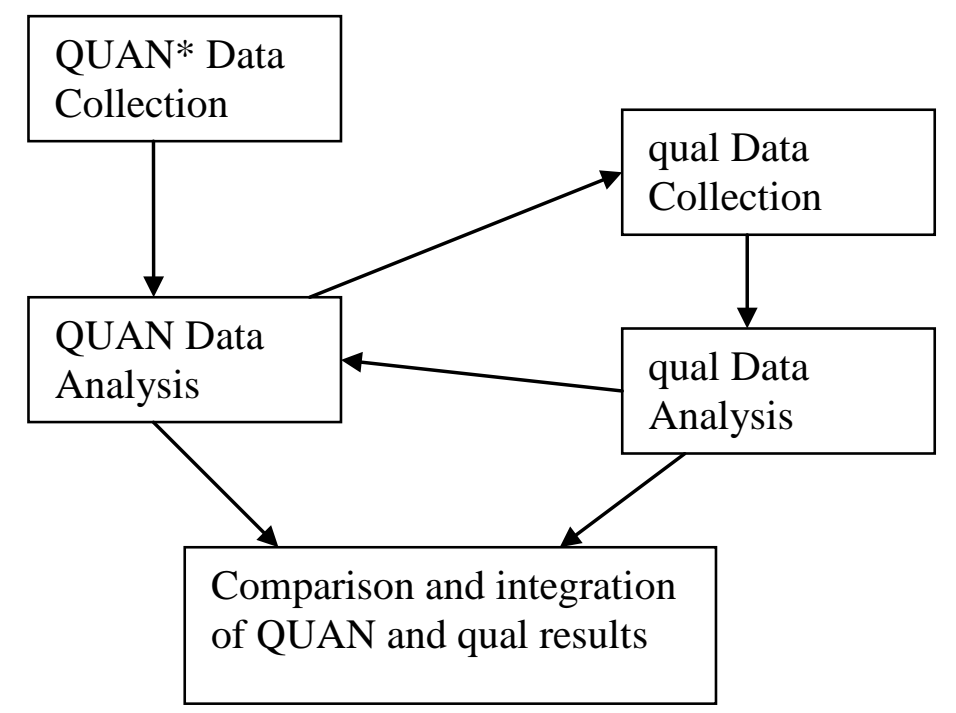

Figure 2 Visual Model for Mixed Methods Design

*According to Creswell et al. (2003) and Morse's (1991) notational system, the uppercase letter suggest major emphasis, while the lowercase letters imply less emphasis. 


\section{Chapter 8}

\section{Survey - Method}

\subsection{Participants}

The survey was carried out during the period from May to November 2007. Participants were recruited, with the help of a parish worker or social worker, from four congregations and one social centre. More than seven hundred questionnaires were distributed, and the response rate varied across the settings because participation in the survey was voluntary. A total of 324 questionnaires were collected and the overall response rate was $45.3 \%$ (see Table 1 ).

Table 1 Questionnaire response

\begin{tabular}{lccccc}
\hline Congregation & FDC & CHC & SCC & EVC & Social centre \\
\hline Collected questionnaires & 60 & 69 & 44 & 94 & 57 \\
Response rate (\%) & 75 & 96 & 55 & 29 & 38 \\
\hline
\end{tabular}

Overall, 132 males and 187 females participated (5 did not specify) in the present study. The mean age of participants was 37.39 years (ranging between 16-78 years of age). While $48.1 \%$ of participants were single, $45.7 \%$ were married and $5.2 \%$ were divorced or widowed. Regarding education, respondents with senior secondary level education or above were the largest group: over $50 \%$ of respondents. Therefore, respondents in the sample had an overall higher education level than Hong Kong overall, with $24.3 \%{ }^{7}$ of the general Hong Kong population with senior secondary level education or above. The median monthly income level of the sample ranged from 10,000 HKD to just under 20,000 HKD. A total of 274 respondents claimed to be Protestant Christian, 48 participants claimed another religion or not to be religious. In the congregation sample, Table 2 indicates that the demographic data matches the general makeup of Protestant congregants in Hong Kong: female dominated with the majority of members aged around 25-44, of middle income, and with medium to high education levels (Wu \& Fok, 2006).

\footnotetext{
${ }^{7}$ http://www.statistics.gov.hk/hk_in_figures/educ/educ2c.html
} 
Table 2 Comparison of present study to Hong Kong population data

\begin{tabular}{|c|c|c|c|c|c|}
\hline & & $\begin{array}{l}\text { Studied } \\
\text { congregation }\end{array}$ & $\begin{array}{l}\text { HK church } \\
2004\end{array}$ & $\begin{array}{l}\text { Studied sample } \\
\text { (including } 4 \\
\text { church and } \\
\text { social centre) }\end{array}$ & $\begin{array}{l}\text { HK population } \\
2004\end{array}$ \\
\hline \multicolumn{6}{|l|}{ Sex } \\
\hline & Male & $40.5 \%$ & $38.5 \%$ & $41.4 \%$ & $48.2 \%$ \\
\hline & Female & $59.5 \%$ & $61.5 \%$ & $58.6 \%$ & $51.8 \%$ \\
\hline \multicolumn{6}{|l|}{ Age } \\
\hline & $0-14$ & $0 \%$ & $14.1 \%$ & $0 \%$ & $15.1 \%$ \\
\hline & $15-24$ & $10.5 \%$ & $16.1 \%$ & $20.1 \%$ & $13.1 \%$ \\
\hline & $25-44$ & $42.3 \%$ & $36.5 \%$ & $36.7 \%$ & $35 \%$ \\
\hline & $45-64$ & $32.2 \%$ & $21.5 \%$ & $29 \%$ & $24.9 \%$ \\
\hline & $\geq 65$ & $15 \%$ & $11.9 \%$ & $14.2 \%$ & $11.9 \%$ \\
\hline
\end{tabular}

Nevertheless, looking in depth into each participating organization, the demographic findings related to each organization reveal more information about the unique characteristics of each setting (see Table 3).

The findings show that there are no significant gender differences in churches and social centres. The participants in EVC appear older than the other groups, while those in the social centre are the youngest. Churchgoers, particularly members of EVC and SCC, are generally more educated and earn higher incomes than the non-religious group (see section 9.2.2).

\subsection{Instruments}

The questionnaires included measures of various dimensions of spirituality, perception of community, and well-being outcomes. In addition, respondents' social beliefs and demographics were explored. All the scales were translated into Chinese, with the assistance of a third person who had a qualification in translation, to ensure the meaning of the measurement was correct (see Appendix 1 for details of the questionnaire).

Regarding spirituality, several scales were used to measure belief, experience and practice related to spirituality. 
Table 3 Demographic data of studied settings

\begin{tabular}{|c|c|c|c|c|c|}
\hline & Social centre \% & $\mathrm{CHC} \%$ & SCC \% & FDC \% & EVC \% \\
\hline \multicolumn{6}{|l|}{ Sex } \\
\hline Male & 45.6 & 30.3 & 45.5 & 40 & 45.2 \\
\hline Female & 54.4 & 69.7 & 54.5 & 60 & 54.8 \\
\hline \multicolumn{6}{|l|}{ Age } \\
\hline 20 or below & 54.9 & 3.9 & 7.7 & 5.8 & 0 \\
\hline $21-30$ & 19.6 & 17.7 & 43.6 & 13.4 & 23 \\
\hline $31-40$ & 5.9 & 35.3 & 7.7 & 32.7 & 20.7 \\
\hline $41-50$ & 17.6 & 33.3 & 15.4 & 36.6 & 25.3 \\
\hline $51-60$ & 2 & 9.8 & 23 & 11.5 & 27.6 \\
\hline$>60$ & 0 & 0 & 2.6 & 0 & 3.4 \\
\hline \multicolumn{6}{|l|}{ Education } \\
\hline Primary School & 1.8 & 4.5 & 0 & 1.7 & 5.4 \\
\hline Junior Secondary & 5.5 & 10.4 & 4.7 & 11.6 & 5.4 \\
\hline Senior Secondary & 69.1 & 41.8 & 25.6 & 31.7 & 11.8 \\
\hline Tertiary education & 16.4 & 9.0 & 18.6 & 13.3 & 16.1 \\
\hline University & 5.5 & 26.9 & 27.9 & 35 & 35.5 \\
\hline Postgraduate & 1.8 & 7.5 & 23.3 & 6.7 & 25.8 \\
\hline \multicolumn{6}{|l|}{ Income } \\
\hline$<\$ 5000$ & 48.7 & 10.2 & 17.6 & 7.5 & 5.4 \\
\hline$\$ 5000-<1000$ & 12.8 & 20.3 & 14.7 & 24.5 & 10.8 \\
\hline$\$ 10000-<20000$ & 17.9 & 45.8 & 38.2 & 28.3 & 27.0 \\
\hline$\$ 20000-<30000$ & 5.1 & 13.6 & 8.8 & 15.1 & 18.9 \\
\hline$\$ 30000-<40000$ & 10.3 & 1.7 & 5.9 & 15.1 & 14.9 \\
\hline$\geq \$ 40000$ & 5.1 & 8.5 & 14.7 & 9.4 & 23.0 \\
\hline \multicolumn{6}{|l|}{ Religion } \\
\hline No religion & 64.9 & 0 & 6.8 & 1.7 & 0 \\
\hline Protestant & 24.6 & 100 & 90.9 & 98.3 & 100 \\
\hline Other religion & 10.5 & 0 & 2.3 & 0 & 0 \\
\hline
\end{tabular}


Religious Fundamentalism Scale. The revised short version Religious Fundamentalism Scale (Altemeyer \& Hunsberger, 2004) was used to measure participants' religious belief, especially their belief about the truth of their religious faith. While the scale is not specific to particular kinds of religious belief, it is suitable for “fundamentalist” Protestant denominations. Examples of the scale included: “God has given humanity a complete unfailing guide to happiness and salvation, which must be totally followed", and "The basic cause of evil in this world is Satan, who is still constantly and ferociously fighting against God”. The reliability and validity of the original 20 items, and the shortened religious fundamentalism scale of 12 items, are discussed in the Altemeyer and Hunsberger's study. The alpha coefficient of the scale attained was over 0.9, and the religious fundamentalism scale also highly and positively correlated with right-wing authoritarianism, religious ethnocentrism and dogmatism, thereby confirming its validity. These 12 items were presented on a Likert scale ranging from -4 (strongly disagree) to 4 (strongly agree). Negatively worded questions were reverse scored and then every score was added a constant of 5 (to avoid negative values). Therefore, total score ranged from 12 to 108.

Daily Spiritual Experience Scale. The experiential dimension of spirituality will be explored by the Daily Spiritual Experience Scale (DSES, Underwood \& Teresi, 2002). The DSES scale tapped the ordinary spiritual experience of people and attained good reliability with internal consistency estimates in the 0.9s. Examples of the scale included: “I feel God's presence”, and “I experience a connection to all life”. DSES has been applied and recently translated to Spanish, Korean, Hebrew, French and Vietnamese (Underwood, 2006) and the Chinese version is in progress. Among these 16 items, the first 15 were scored using a modified Likert scale (the response categories are many times a day $=6$, every day $=5$, most days $=4$, some days $=3$, once in a while $=2$, and never or almost never=1), as suggested by Underwood and Teresi (Underwood \& Teresi, 2002). The last item “in general, how close do you feel to God?” has four response categories (not close at all $=1$, somewhat close $=2$, very close $=3$, and as close as possible $=4$ ). The total score ranges from 16 to 94 .

In terms of the participatory dimension of spirituality, both personal religious practice and the congregational participation were explored. The extent of personal religious practice was assessed by asking participants how frequently they attended 
church, or were involved in private prayer and meditation, bible reading, or church missions, on a six point scale. The respondents' level of congregational participation was assessed by a question related to the Hong Kong context. The participants were asked whether they had participated and also asked to estimate the time they spent on 20 major kinds of activities provided by congregations in Hong Kong (Wu \& Fok, 2006), such as fellowship, choir, visitation or evangelical preaching activities. The total number of activities they joined and the time they spent on the activities were examined separately in the analysis. The number of years they had attended church was also assessed.

Perception towards community. The respondents' perception toward their settings was assessed in several ways. Firstly, respondents were asked the extent to which they identified with a particular label (that is, member of their own congregation/centre, Christian/ religious believer, Hong Konger, and Chinese). Secondly, the believerrespondents were asked the extent to which they think their church had a certain orientation (such as, concern about social justice, caring for neighbours, or preaching the gospel). Both questions used the 7 point Likert-scale.

Sense of community Index (SCI). In addition, respondents' sense of community within their immediate setting was examined by using an adapted version of Sense of Community Index (SCI, Perkins, Florin, Rich, Wandersman, \& Chavis, 1990). Examples of the scale included: “It is very important to me to belong to this particular church", and “I feel at home at this church". It was expected the respondents' relationship towards the congregation be revealed. The psychometric properties of dimensions of SCI are still under much debate; however, the total SCI index has been found to be reliable (Long \& Perkins, 2003; Obst \& White, 2004; N. A. Peterson, Speer, \& Hughey, 2006), and SCI is used in this study to measure people's subjective sense of community in the immediate context: religious setting, and social centre. These 12 items were presented on a Likert scale ranging from 1 (strongly disagree) to 7 (strongly agree). Negatively worded questions were reverse scored before analysis. The scale ranges from 12 to 48.

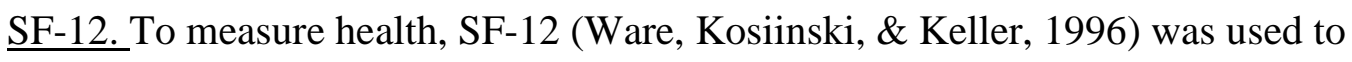
measure the general health. The Chinese version of SF-12 was recently translated and validated (C. L. K. Lam, Tse, \& Gandek, 2005). SF-12 measurement can be divided into two major domains: physical health (PCS), and mental health (MCS). The physical health component is mainly associated with physical functioning, role physical and bodily pain 
(examples of questions included: "During the past 4 weeks, how much did pain interfere with your normal work?”, and “Does your health limit you in certain kind of activities?”) The mental health component correlated strongly with mental health, emotional role, and social functioning (examples of questions included: “Have you felt calm and peaceful?”, and "have you felt downhearted and depressed"). The data from both domains - PCS and MCS scores - were examined separately and calculated by the standard algorithm described in the SF-12 v.2 Manual (Ware, Kosinski, Turner-Bowker, \& Gandeck, 2002).

Satisfaction with Life Scale. Satisfaction with Life Scale (SWLS) (Diener, Emmons, Larsen, \& Griffen, 1985) was used to measure the respondents’ overall subjective evaluation of life satisfaction. The SWLS have been widely applied and validated across nations, including with the Chinese population (Sachs, 2003). Examples of items included: "In most ways my life is close to my ideal”, and "The conditions of my life are excellent”. The five items were presented on a Likert scale ranging from 1 (strongly disagree) to 7 (strongly agree). The scale ranged from 5 to 35.

Social Trust and Civic participation. The participants’ level of social trust was assessed by nine items (three items of each measuring general social trust, trust in coworkers and trust in neighbours respectively), adapted from Welch et al. (2004). Examples of the items included: "Most of the time people try to be helpful", and "most people try to take advantage of you”. These nine items were presented on a Likert scale ranging from 1 (strongly disagree) to 5 (strongly agree). The negative worded questions were reverse scored before analysis. The scale ranged from 9 to 45 .

Regarding civic participation, respondents faced four items, assessing how often they participated in voluntary organizations (volunteering and serving on any committee), reading newspapers, and following current and political affairs. These four items were also present on a modified Likert scale (Never $=1$, seldom $=2$, sometimes $=3$, often $=4$, always=5). The scale ranged from 4 to 20 .

Furthermore, membership of voluntary organizations was also measured by a question asking "How many voluntary associations, other than your church/social centre, do you belong to or are affiliated with?”.

Social Axiom Survey. Regarding the cultural dimension, 25 items of the social axiom survey (SAS, Leung \& Bond, 2004) was used to tap general social beliefs, in five dimensions, including social cynicism (an example included: "Powerful people tend to 
exploit others”); reward for application (an example included: "Every problem has a solution”); social flexibility (an example included: "Human behaviour changes with the social context”); control by fate (an example included: "Fate determines one’s success and failure”); and religiosity (an example included: 'Religious faith contributes to good mental health"). The scale can be conducted at the individual level and had been widely applied (Leung \& Bond, 2004; Singelis, Hubbard, Her, \& An, 2003). The reliability and validity of SAS was satisfactory (The average alphas of the 25 items version for the Hong Kong sample was 0.76 , Chen, Bond, \& Cheung, 2006). Negative worded questions were reverse scored before analysis. The scale of each dimension ranged from 5 to 25 .

\subsection{Procedure}

Permission from participating congregations and social centre to carry out the present study from was sought in early 2007. To minimize disturbing the normal operation of the organisations, the researcher carefully negotiated with the organizations. With the help of the parish or social workers, the questionnaires were distributed at the Sunday service, fellowships meetings, or some relevant functional groups. People were invited to take part in the study after their original planned activities. Although the researcher was present at two congregations (social concern and charismatic church) to answer any queries, basically the questionnaires were left to parish or social workers to distribute and collect according to their schedule. Participation in the survey was voluntary and the purpose and the (voluntary) nature of the study were clearly stated in covering letters. The consent of the participants was assumed by returned questionnaires.

It is worth noting two things about the data collection process. The first is the ease in conducting the survey in the charismatic church ( $\mathrm{CHC}$ ), while the second is the difficulty faced in liaising with the evangelical church (EVC). In the charismatic church, the survey was conducted after Sunday bible classes which more than 100 members attended. At the request of the pastor, unexpectedly nearly all the people stayed to fill in the questionnaires, more than the number of questionnaires prepared by the researcher. In contrast, in the evangelical church, given that the approval was gained from the pastor in charge, conducting the survey was still difficult, if not impossible, because of the size, (over 5000 members) of EVC. Whom to recruit and how to recruit became critical issues that nearly spoiled the study. The EVC is a church with a hierarchical structure and clear 
division of labour in pastoral work, and it was a challenging task to reach the huge population of the EVC, particularly when the liaising pastor, who was responsible for only a few fellowships in a young adult ministry and one specific ministry, was only one of the nearly 50 pastors in the church. Therefore, to reach the wider population of the church, the researcher had to make use of his personal network to be introduced to four other pastors (two of which were senior pastors) who were responsible for the adult ministry, men's ministry, church music ministry, and young adult ministry. Finally, the questionnaires were distributed to the four fellowships in the young adult ministry, adult ministry, men and couple ministry, and one choir team out of five. As requested by the pastor who was in charge of the adult ministry, the questionnaires distributed to the adult fellowships had return envelopes attached so that the finished questionnaires could be sent back to the researcher directly. For the other fellowships or ministry, the researcher was fortunate to have the assistance of key people who helped to distribute and collect the questionnaires. 


\section{Chapter 9}

\section{Survey - Results}

This research was designed to test the impact of both spirituality and religious community on people's well-being. For the basic sets of analyses, the five settings represented the main independent variable, and spirituality and well-being the key dependent variables. It was anticipated that complex, rather than simple linear relationships would be found, and a series of analyses ranging from t-tests and ANOVAs to regressions and discriminant function analyses were completed. Finally, path analysis was conducted to test direct and mediating effects of the combined variables.

\subsection{Internal reliability}

Many of the tests used in the data collection have established psychometrics in non-Chinese populations. In order to determine their utility with the current sample, reliability analyses were conducted. The Cronbach's alphas for each scale are presented in Table 4 Cronbach's alphas for the scales

\begin{tabular}{lllc}
\hline Scale (no. of items) & Male & Female & Total sample \\
\hline $\begin{array}{l}\text { Satisfaction with Life Scale (5 items) } \\
\text { Trust scale }\end{array}$ & 0.847 & 0.864 & 0.855 \\
Total Scale (9 items) & 0.746 & 0.755 & 0.751 \\
Generalized trust (3 items) & 0.476 & 0.538 & 0.516 \\
Trust towards colleague (3 items) & 0.736 & 0.781 & 0.759 \\
Trust towards neighbor (3 items) & 0.475 & 0.493 & 0.483 \\
Fundamentalism Scale (12 items) & 0.890 & 0.846 & 0.863 \\
Daily Spiritual Experience Scale (16 items) & 0.961 & 0.962 & 0.962 \\
Sense of Community Index (12 items) & 0.709 & 0.720 & 0.716 \\
Social Belief Scale (25 items) & & & \\
Social Cynicism (5 items) & 0.701 & 0.633 & 0.668 \\
Reward for Application (5 items) & 0.698 & 0.632 & 0.667 \\
Social Flexibility(5 items) & 0.671 & 0.534 & 0.608 \\
Fate control (5 items) & 0.631 & 0.622 & 0.626 \\
Spirituality (5 items) & 0.801 & 0.686 & 0.744 \\
\hline
\end{tabular}

The reliability measure for each of the scales was satisfactory. The Satisfaction with Life Scale (SWLS), Fundamentalism Scale, and Daily Spiritual Experience Scale 
had an alpha of $0.855,0.863$, and 0.962 respectively for the total sample, which are strong coefficients.

Two of the three sub-scales of the Trust Scale had quite low reliabilities, with generalized trust being 0.52 and trust towards neighbour being 0.48 . As the nine-item Trust scale attained a Cronbach’s alpha (0.75) much higher than the three subscales, with three items in each domain, the total trust score was used instead.

\subsection{Descriptives}

\subsubsection{Key variables across gender}

Means and standard deviations were calculated for the key major variables (see Table 5) and four transformed variables "Number of voluntary organizations joined”, “SF12 -- physical health dimension”, "Number of years attending church” and "Time (number of hours) spent on church activities". The four variables were transformed for further analyses because of the violation of the normality assumption. According to Tabachnick and Fidell (2007), different ways of transformation of the variables may be used depending on the extent to which they diverge from normal. For example, in this study, an inverse transformation was used on the number of affiliated organizations joined because of severe violation, while a "reflect and square root" transformation $^{8}$ was applied on the measure of physical health because of the negative skewness. A square root transformation was applied to the number of years of attending church and a logarithmic transformation was used on the time spent on church activities because of their moderate to substantial violation of normality assumptions. One variable, identification as the member of the setting, was negatively skewed without transformation.

Simple independent sample t-tests were conducted across all samples pooled together for the major variables to examine whether there were differences between the sexes (Table 5). The results indicated most of these means were not significantly different, except the physical health dimension and the measure of social cynicism. In both cases,

\footnotetext{
${ }^{8}$ The inverse transformation (Field, 2005) and reflected variable (Tabachnick \& Fidell, 2007) have the effect of reversing the score; therefore, as Andy, Tabachnick and Fidell suggest, the direction was reversed in the interpretation.
} 
Table 5 Mean and Standard Deviations of variables among Males and Females and the Total sample



\footnotetext{
\# As mentioned before, because of the inverse and reflected transformation of the variable, the direction should be reversed in the interpretation.

$* p<.05$
} 
the male participants also had a significantly higher score than their female counterparts. Specifically, males $(\mathrm{M}=3.46, \mathrm{SD}=0.92)$ had a significantly higher mean in physical health than females $(\mathrm{M}=3.73, \mathrm{SD}=0.99), \mathrm{t}(308)=-2.43, \mathrm{p}=.016$. Males $(\mathrm{M}=17.2, \mathrm{SD}=$ 3.09) had a significantly higher mean level of social cynicism than their female counterparts $(\mathrm{M}=16.46, \mathrm{SD}=2.85), \mathrm{t}(308)=2.17, \mathrm{p}=.031$.

\subsubsection{Education and income across settings}

Chi-square tests were conducted to examine any significant differences between the settings and the members' educational level and monthly income. The sample was divided into three educational levels: low (primary or junior secondary level); medium (senior secondary level or post-secondary level); and high (university or post-graduate level). Income was also divided into three levels: low (up to 10,000 HKD per month); medium (from 10,000 to 30,000 HKD); and high (above 30,000 HKD).

Table 6 and 7 demonstrate that there is a significant association between settings, educational level, and monthly income earned by participants. With respect to educational level, more than half of the SCC and EVC participants had high educational levels, while the majority of participants in the social centre, CHC had FDC had medium educational levels. Regarding income, over half of the participants in the social centre earned low levels of income, while those belonging to the church had medium to high incomes. Cramer's V value indicates a medium strength of association.

Table 6 Chi square test of settings by educational level

\begin{tabular}{lcccccc}
\hline $\mathrm{N}=318$ & \multicolumn{5}{c}{ Educational level } \\
Settings & Soc Center & CHC & SCC & FDC & EVC & Total \\
\hline Low & $4(7.3 \%)$ & $10(14.9 \%)$ & $2(4.7 \%)$ & $8(13.3 \%)$ & $10(10.8 \%)$ & $34(10.7 \%)$ \\
Medium & $47(85.5 \%)$ & $34(50.7 \%)$ & $19(44.2 \%)$ & $27(45 \%)$ & $26(28 \%)$ & $153(48.1 \%)$ \\
High & $4(7.3 \%)$ & $23(34.3 \%)$ & $22(51.2 \%)$ & $25(41.7 \%)$ & $57(61.3 \%)$ & $131(41.2 \%)$ \\
Total & $55(17.3 \%)$ & $67(21.1 \%)$ & $43(13.5 \%)$ & $60(16.9 \%)$ & $93(29.2 \%)$ & $318(100 \%)$ \\
\hline$\chi 2(8)=54.02, p<0.001$, Cramer's V=.291
\end{tabular}

Table 7 Chi square test of settings by monthly income

\begin{tabular}{|c|c|c|c|c|c|c|}
\hline \multirow{2}{*}{$\begin{array}{l}\mathrm{N}=259 \\
\text { Settings }\end{array}$} & \multirow[b]{2}{*}{ Soc Center } & \multicolumn{3}{|c|}{ Monthly Income } & \multirow[b]{2}{*}{ EVC } & \multirow[b]{2}{*}{ Total } \\
\hline & & $\mathrm{CHC}$ & SCC & FDC & & \\
\hline Low & $24(61.5 \%)$ & $18(30.5 \%)$ & 11(32.4\%) & $17(32.1 \%)$ & $12(16.2 \%)$ & 82(31.7\%) \\
\hline Medium & $9(23.1 \%)$ & $35(59.3 \%)$ & $16(47.1 \%)$ & $23(43.4 \%)$ & $34(45.9 \%)$ & $117(45.2 \%)$ \\
\hline High & $6(15.4 \%)$ & $6(10.2 \%)$ & $7(20.6 \%)$ & $13(24.5 \%)$ & $28(37.8 \%)$ & $60(23.2 \%)$ \\
\hline Total & 39(15.1\%) & $59(22.8 \%)$ & $34(13.1 \%)$ & $53(20.5 \%)$ & 74(28.6\%) & $259(100 \%)$ \\
\hline
\end{tabular}




\subsubsection{Religious practices across settings}

The association between settings and several categorical variables of religious practices is shown in Tables 8-11. They are: prayer and meditation, bible reading, serving in church, and church attendance. The variables were divided into two levels: low (participants who undertake the practice less than once per month), and high (participants who practice monthly or more). As expected, Chi-square tests indicate that there are also significant differences in the following four religious practices across settings.

Table 8 Chi square test of settings by prayer and meditation

\begin{tabular}{lcccccc}
\hline $\begin{array}{l}\text { N= 311 } \\
\text { Settings }\end{array}$ & Soc Center & CHC & SCC & FDC & EVC & Total \\
\hline $\begin{array}{l}\text { Low (less than } \\
\text { monthly) }\end{array}$ & $30(55.6 \%)$ & $1(1.5 \%)$ & $6(14 \%)$ & $2(3.4 \%)$ & $2(2.2 \%)$ & $41(13.2 \%)$ \\
$\begin{array}{l}\text { High } \\
\text { (monthly or more) }\end{array}$ & $24(44.4 \%)$ & $64(98.5 \%)$ & $37(86 \%)$ & $56(96.6 \%)$ & $89(97.8 \%)$ & $270(86.8 \%)$ \\
\begin{tabular}{l} 
Total \\
\hline$\chi^{2}(4)=106.83, p<0.001$, Cramer's V=.586)
\end{tabular} & $43(13.8 \%)$ & $58(18.6 \%)$ & $91(29.3 \%)$ & $311(100 \%)$ \\
\hline
\end{tabular}

Table 9 Chi square test of settings by bible reading

\begin{tabular}{lcccccc}
\hline $\begin{array}{l}\mathrm{N}=310 \\
\text { Settings }\end{array}$ & Soc Center & CHC & SCC & FDC & EVC & Total \\
\hline $\begin{array}{l}\text { Low (less than } \\
\text { monthly) }\end{array}$ & $40(74.1 \%)$ & $2(3.1 \%)$ & $9(20.9 \%)$ & $1(1.7 \%)$ & $1(1.1 \%)$ & $53(17.1 \%)$ \\
$\begin{array}{l}\text { High } \\
\text { (monthly or more) }\end{array}$ & $14(25.9 \%)$ & $63(96.9 \%)$ & $34(79.1 \%)$ & $57(98.3 \%)$ & $89(98.9 \%)$ & $257(82.9 \%)$ \\
Total & $54(17.4 \%)$ & $65(21 \%)$ & $43(13.9 \%)$ & $58(18.7 \%)$ & $90(29 \%)$ & $310(100 \%)$ \\
\hline
\end{tabular}
$\chi^{2}(4)=159.04, \mathrm{p}<0.001$, Cramer’s $\mathrm{V}=.716$

Table 10 Chi square test of settings by serving in the church

\begin{tabular}{lcccccc}
\hline $\begin{array}{l}\text { N= 309 } \\
\text { Settings }\end{array}$ & Soc Center & CHC & SCC & FDC & EVC & Total \\
\hline $\begin{array}{l}\text { Low (less than } \\
\text { monthly) }\end{array}$ & $40(74.1 \%)$ & $7(10.8 \%)$ & $6(14 \%)$ & $14(24.1 \%)$ & $15(16.9 \%)$ & $82(26.5 \%)$ \\
$\begin{array}{l}\text { High } \\
\text { (monthly or more) }\end{array}$ & $14(25.9 \%)$ & $58(89.2 \%)$ & $37(86 \%)$ & $44(75.9 \%)$ & $74(83.1)$ & $227(73.5 \%)$ \\
$\begin{array}{l}\text { Total } \\
\chi^{2}(4)=78.83, p<0.001, \text { Cramer's V=.505 }\end{array}$ & $43(13.9 \%)$ & $58(18.8 \%)$ & $89(28.8 \%)$ & $309(100 \%)$ \\
\hline
\end{tabular}


Table 11 Chi square test of settings by church attendance

\begin{tabular}{|c|c|c|c|c|c|c|}
\hline \multirow{2}{*}{$\begin{array}{l}\mathrm{N}=311 \\
\text { Settings }\end{array}$} & \multicolumn{6}{|c|}{ Church attendance } \\
\hline & Soc Center & $\mathrm{CHC}$ & SCC & FDC & EVC & Total \\
\hline $\begin{array}{l}\text { Low (less than } \\
\text { monthly) }\end{array}$ & 41 (74.5\%) & $1(1.5 \%)$ & $3(7 \%)$ & $1(1.8 \%)$ & 1 (1.1\%) & 47 (15.1\%) \\
\hline $\begin{array}{l}\text { High } \\
\text { (monthly or more) }\end{array}$ & $14(25.5 \%)$ & 64 (98.5\%) & 40 (93\%) & 56 (98.2\%) & 90 (98.9\%) & $264(84.9 \%)$ \\
\hline Total & 55 (17.7\%) & 65 (20.9\%) & 43 (13.8\%) & 57 (18.3\%) & $91(29.3 \%)$ & $311(100 \%$ \\
\hline
\end{tabular}

The findings indicate that there is a significant association between settings and religious practice in the four dimensions. Cramer's V value confirms the association is strong, particularly regarding church attendance.

Comparing the four religious settings (Table 12-15), the variables were recategorized in two levels: low (participants who practice less than once per week) and high (participants who practice weekly or more). The chi-square test indicates significant association between the four churches in prayer, meditation, and reading the bible, but not in serving in the church. Here, members of the social concern church practise less frequently than members of the other three churches. Moreover, there was a significant association between church settings and church attendance, but note there are two cells (25\%) of expected frequencies below 5 , resulting in a loss of statistical power.

Table 12 Chi square test of settings by prayer and meditation

\begin{tabular}{|c|c|c|c|c|c|}
\hline \multirow{2}{*}{$\begin{array}{l}\mathrm{N}=257 \\
\text { Settings }\end{array}$} & \multicolumn{5}{|c|}{ Prayer and meditation } \\
\hline & $\mathrm{CHC}$ & SCC & FDC & EVC & Total \\
\hline $\begin{array}{l}\text { Low (less than } \\
\text { weekly) }\end{array}$ & $3(4.6 \%)$ & 17 (39.5) & $4(13.3 \%)$ & $6(6.6 \%)$ & $30(11.7 \%)$ \\
\hline $\begin{array}{l}\text { High } \\
\text { (weekly or more) }\end{array}$ & 62 (95.4\%) & $26(60.5 \%)$ & 54 (93.1\%) & 85 (93.4\%) & 227(88.3\%) \\
\hline Total & $65(25.3 \%)$ & $43(16.7 \%)$ & $58(22.6 \%)$ & $91(35.4 \%)$ & 257 (100\%) \\
\hline
\end{tabular}

Table 13 Chi square test of settings by bible reading

\begin{tabular}{lccccl}
\hline $\begin{array}{l}\mathrm{N}=256 \\
\text { Settings }\end{array}$ & CHC & SCC & FDC & EVC & Total \\
\hline $\begin{array}{l}\text { Low (less than } \\
\text { weekly) }\end{array}$ & $5(7.7 \%)$ & $27(62.8 \%)$ & $10(17.2 \%)$ & $13(14.4 \%)$ & $55(21.5 \%)$ \\
$\begin{array}{l}\text { High } \\
\text { (weekly or more) }\end{array}$ & $60(92.3 \%)$ & $16(37.2 \%)$ & $48(82.8 \%)$ & $77(85.6 \%)$ & $201(78.5 \%)$ \\
Total & $65(25.4 \%)$ & $43(16.8 \%)$ & $58(22.7 \%)$ & $90(35.2 \%)$ & $256(100 \%)$ \\
\hline$\chi^{2}(3=54.09, \mathrm{p}<0.001$, Cramer's $\mathrm{V}=.46, \mathrm{p}<.001$ &
\end{tabular}


Table 14 Chi square test of settings by serving in the church

\begin{tabular}{|c|c|c|c|c|c|}
\hline \multirow{2}{*}{$\begin{array}{l}\mathrm{N}=255 \\
\text { Settings }\end{array}$} & \multicolumn{5}{|c|}{ Serving in the church } \\
\hline & $\mathrm{CHC}$ & SCC & FDC & EVC & Total \\
\hline $\begin{array}{l}\text { Low (less than } \\
\text { weekly) }\end{array}$ & $29(44.6 \%)$ & $21(48.8 \%)$ & $25(43.1 \%)$ & $31(34.8 \%)$ & 106(41.6\%) \\
\hline $\begin{array}{l}\text { High } \\
\text { (weekly or more) }\end{array}$ & $36(55.4 \%)$ & $22(51.2)$ & $33(56.9 \%)$ & $58(65.2 \%)$ & $149(58.4 \%)$ \\
\hline Total & $65(25.5 \%)$ & $43(16.9 \%)$ & $58(22.7 \%)$ & 89(34.9\%) & 255 (100\%) \\
\hline \multicolumn{6}{|c|}{$\chi^{2}(3)=2.9, p^{>} .05$, Cramer's V $=.107, p^{>} .05$} \\
\hline \multicolumn{6}{|c|}{ Table 15 Chi square test of settings by church attendance } \\
\hline $\mathrm{N}=256$ & & & hurch attenda & ince & \\
\hline Settings & $\mathrm{CHC}$ & SCC & FDC & EVC & Total \\
\hline $\begin{array}{l}\text { Low (less than } \\
\text { weekly) }\end{array}$ & $1(1.5 \%)$ & $15(34.9 \%)$ & $2(3.5 \%)$ & $3(3.3 \%)$ & $21(8.2 \%)$ \\
\hline $\begin{array}{l}\text { High } \\
\text { (weekly or more) }\end{array}$ & 64 (98.5\%) & $28(65.1 \%)$ & 55 (96.5\%) & $88(96.7 \%)$ & 235(91.8\%) \\
\hline Total & $65(25.4 \%)$ & $43(16.8 \%)$ & $57(22.3 \%)$ & $91(35.5 \%)$ & $256(100 \%)$ \\
\hline
\end{tabular}

\subsubsection{Participation in voluntary organizations across settings}

Chi-square tests measured any significant associations between the settings and members' affiliation with voluntary organization outside their setting. The sample was divided into two levels: no affiliation (participants not affiliated with any other organizations); and with affiliation (participants affiliated with other organizations).

Table 16 indicates a significant association between the settings and voluntary organization affiliation. More than half of SCC members are affiliated with organizations outside church; meanwhile, less than $30 \%$ of CHC members are affiliated with organizations outside church. Nevertheless, Cramer's V value indicates a weak strength of association.

Table 16 Chi square test of the setting by participation in voluntary organization

\begin{tabular}{lcccccc}
\hline N=317 & \multicolumn{6}{c}{ Voluntary organization affiliation } \\
Settings & Soc Center & CHC & SCC & FDC & EVC & Total \\
\hline No & $30(52.6 \%)$ & $49(72.1 \%)$ & $16(37.2 \%)$ & $35(61.4 \%)$ & $55(59.8 \%)$ & $185(58.4 \%)$ \\
Yes & $27(47.4 \%)$ & $19(27.9 \%)$ & $27(62.8 \%)$ & $22(38.6 \%)$ & $37(40.2 \%)$ & $132(41.6 \%)$ \\
Total & $57(18 \%)$ & $68(21.5 \%)$ & $43(13.6 \%)$ & $57(18 \%)$ & $92(29 \%)$ & $317(100 \%)$
\end{tabular}

$\chi^{2}(4)=14.23, p<0.01$, Cramer's V=.212 
Table 17 shows that detail of affiliation of organization across the settings.

Table 17 Type of voluntary organization affiliation across settings

\begin{tabular}{lccccc}
\hline Type of affiliated organization & Social centre & CHC & SCC & FDC & EVC \\
\hline Social service Groups & 15 & 7 & 11 & 7 & 5 \\
Professional association & 2 & 5 & 6 & 5 & 14 \\
Environmental Groups & 0 & 1 & 0 & 0 & 0 \\
Youth work & 11 & 3 & 3 & 0 & 2 \\
Community based Assoc & 5 & 2 & 4 & 0 & 3 \\
Health Groups & 2 & 2 & 3 & 1 & 0 \\
Recreation groups & 8 & 0 & 1 & 1 & 6 \\
Senior Citizen groups & 0 & 3 & 3 & 2 & 1 \\
Women Groups & 1 & 2 & 3 & 4 & 1 \\
Political groups & 1 & 0 & 2 & 0 & 2 \\
Education, art and culture Groups & 2 & 3 & 8 & 3 & 7 \\
Support Groups & 3 & 2 & 1 & 1 & 3 \\
Other association & 6 & 3 & 3 & 3 & 10 \\
\hline
\end{tabular}

\subsubsection{Participation in church activities across settings}

A Chi-square test measured any significant association between the settings and members' participation in church activities. The sample was divided into two levels: without participation in other church activities (participants who did not participate in any of the 20 kinds of church activities in the questionnaire); and participation in church activities (participants who did participate in any of the 20 kinds of church activities in the questionnaire).

Table 18 indicates that there is a significant, but small, association between the settings and members’ participation in church activities. Generally speaking, about $90 \%$ of church members participate in church activities. Nearly $90 \%$ of CHC and SCC members participate in church activities, and over 95\% FDC and EVC members participate in church activities.

Table 18 Chi square test of settings by church activities participation

\begin{tabular}{lccccc}
\hline N=255 & \multicolumn{5}{c}{ Church activities participation } \\
Settings & CHC & SCC & FDC & EVC & Total \\
\hline No & $7(10.9 \%)$ & $6(14 \%)$ & $2(3.4 \%)$ & $2(2.2 \%)$ & $17(6.7 \%)$ \\
Yes & $57(89.1 \%)$ & $37(86 \%)$ & $56(96.6 \%)$ & $88(97.8 \%)$ & $238(93.3 \%)$ \\
Total & $64(25.1 \%)$ & $43(16.9 \%)$ & $58(22.7 \%)$ & $90(35.3 \%)$ & $255(100 \%)$ \\
\hline
\end{tabular}

$\chi 2(4)=9.37, \mathrm{p}<0.05$, Cramer's $\mathrm{V}=.192$ 
Table 19 shows the detail of participation of members in different kinds of church activities across the settings.

Table 19 Type of church activities participated by 4 church members

\begin{tabular}{lccccr}
\hline & \multicolumn{5}{c}{ Church } \\
Type of members' attended church activities & CHC & SCC & FDC & EVC & \multirow{2}{*}{ Total } \\
\hline Fellowship & 45 & 32 & 55 & 82 & 214 \\
Nurturing new believer prog & 13 & 0 & 8 & 12 & 33 \\
Personal evangelical preaching & 11 & 0 & 10 & 9 & 30 \\
Missionary & 5 & 0 & 4 & 6 & 15 \\
Church planting/ church development & 0 & 0 & 1 & 2 & 3 \\
Sunday bible school teaching & 13 & 11 & 11 & 17 & 52 \\
Mentor or leader in the fellowship & 6 & 10 & 23 & 24 & 63 \\
Singsparation/ worship team & 11 & 1 & 3 & 7 & 22 \\
Choir & 6 & 6 & 7 & 16 & 35 \\
Service leader & 0 & 3 & 9 & 3 & 15 \\
Prayer team & 11 & 5 & 19 & 20 & 55 \\
Meditation training & 13 & 1 & 16 & 9 & 39 \\
Visitation & 9 & 8 & 12 & 12 & 41 \\
Social concern & 2 & 10 & 1 & 5 & 18 \\
Church leader training/ meeting & 0 & 2 & 4 & 1 & 7 \\
Discipleship training/ bible study & 5 & 0 & 2 & 4 & 11 \\
Group leader training & 3 & 0 & 0 & 7 & 10 \\
Theology training & 29 & 1 & 8 & 6 & 44 \\
Counseling & 5 & 1 & 2 & 5 & 13 \\
Spiritual healing & 2 & 0 & 0 & 3 & 5 \\
Others & 7 & 0 & 2 & 5 & 14 \\
\hline
\end{tabular}

\subsubsection{Participants' personal identity across settings}

To understand the extent of various personal identities, aggregate scores of members' personal identification across the settings was reported, using a radar chart provided by Excel 2003. Table 20 and Figure 3 show the participants' personal identity across settings. Overall, participants in church settings tend to identify with their religious belief, while those in the social centre feel a stronger national identity rather than religious faith or immediate settings. Moreover, except for the SCC, members of the other three churches also identify more with their church settings than their own national identity (be that identity Hong Kong or Chinese). Nevertheless, it should be noted that the national identity of participants in church settings is still higher than those in social 
centres. This may suggest that religious identity does not cancel out believers’ national identity. Rather, religious faith provides people another source of identification.

Table 20 Participants' personal identity across settings

\begin{tabular}{llllll}
\hline & Social centre & CHC & SCC & FDC & EVC \\
\hline Church/ center member & 4.04 & 6.08 & 4.86 & 5.86 & 5.7 \\
Religious believer & 4.07 & 6.62 & 5.52 & 6.52 & 6.77 \\
Hong Konger & 5.21 & 5.65 & 5.25 & 5.46 & 5.41 \\
Chinese & 4.61 & 5.4 & 4.66 & 5.37 & 5.23 \\
\hline
\end{tabular}

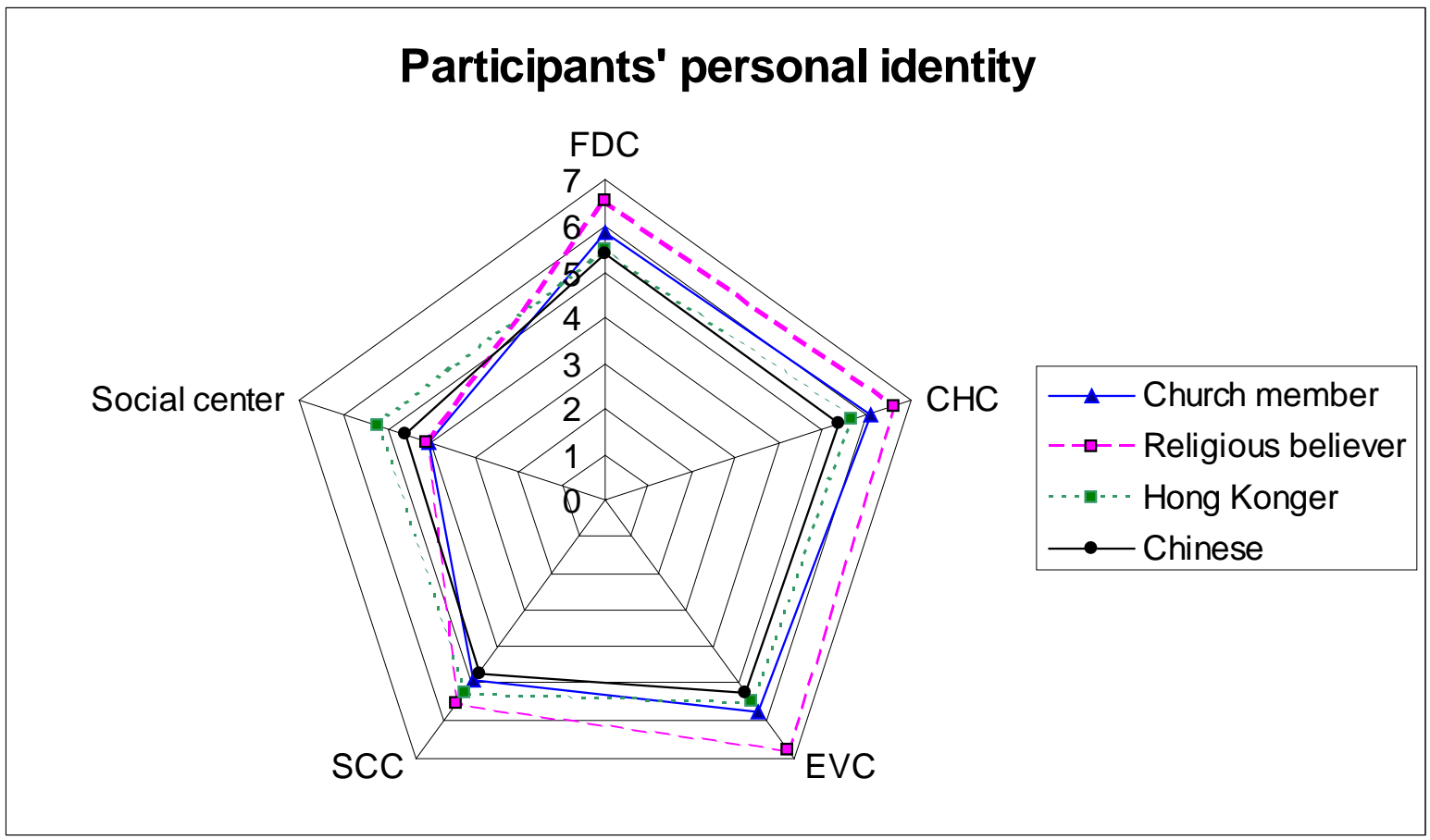

Figure 3 Participants’ personal identity across settings

\subsubsection{Congregational identity perceived by participants across settings}

To understand how church members view their respective churches, the aggregate score of members' perception of their congregation, across the settings, was reported, using a radar chart provided by Excel 2003. Table 21 and Figure 4 show the congregational identity perceived by participants across church settings. The participants in FDC and EVC identify with their church strongest in gospel preaching and building members’ relationship with God. CHC members identified with their church in emphasizing the power of the Holy Spirit, and SCC members identified with their church 
the highest concerning social justice. It should also be noted that ratings by $\mathrm{CHC}$ members on 7 out of 8 congregational identities are also the highest.

Table 21 Congregational identity perceived by members across settings

\begin{tabular}{lcccc}
\hline Congregational identity & CHC & SCC & FDC & EVC \\
\hline Social justice & 6 & 5.62 & 4.85 & 5.68 \\
Care for neighbour & 5.83 & 5.5 & 5.07 & 5.71 \\
Sense of mission & 6.47 & 5 & 5.43 & 6.29 \\
Family type & 5.92 & 4.29 & 5.39 & 5.3 \\
Traditional & 5.8 & 5.15 & 5.61 & 6.01 \\
Relationship with God & 6.55 & 5 & 5.98 & 6.31 \\
Gospel preaching & 6.53 & 4.74 & 5.81 & 6.31 \\
Holy Spirit & 6.71 & 4.5 & 5.19 & 5.7 \\
\hline
\end{tabular}

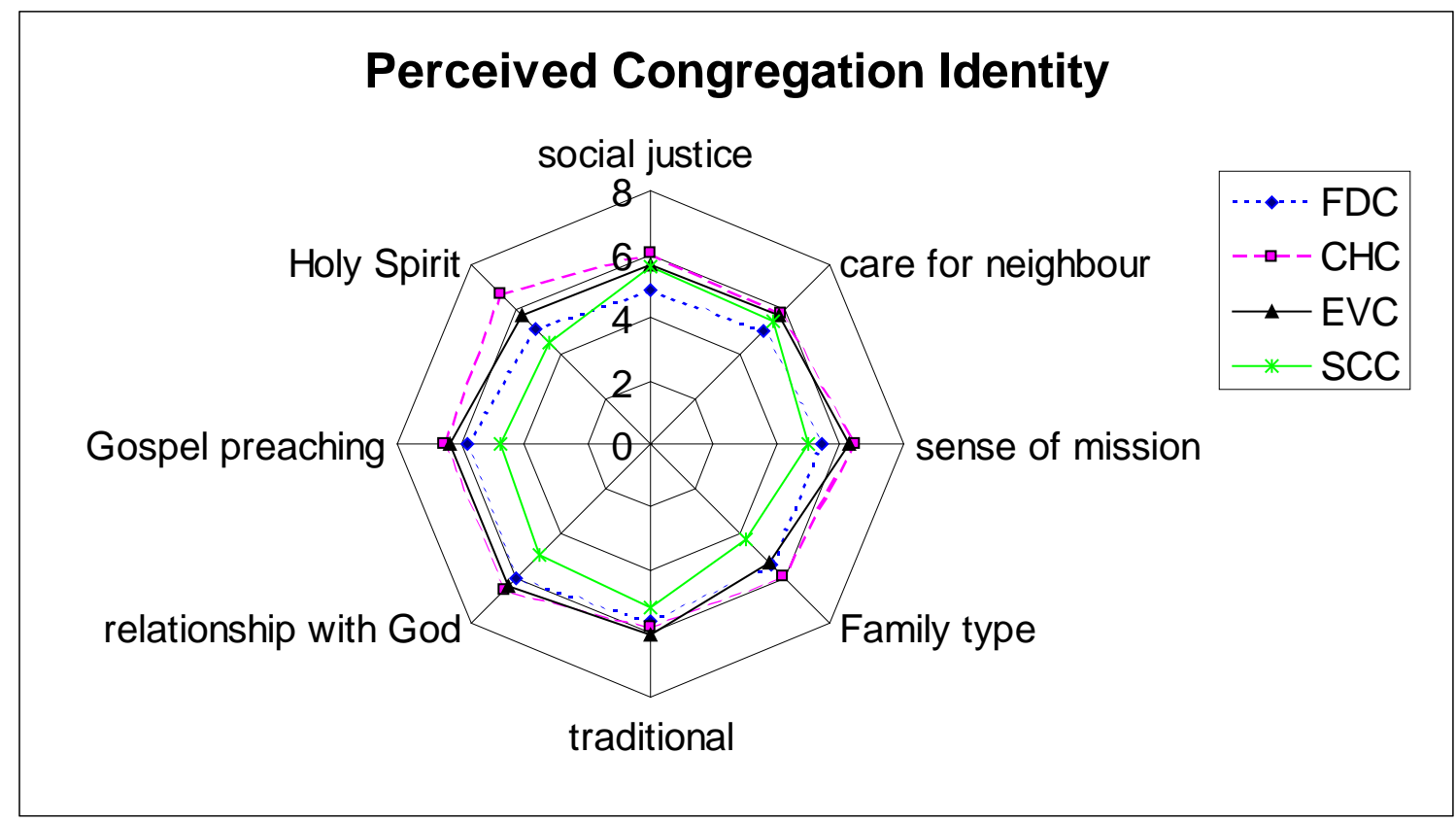

Figure 4 Congregational identity perceived by members across settings

\subsection{The influence of spiritual context}

\subsubsection{Well-being}

\subsubsection{Multivariate analysis of variance}

To examine whether there were differences in well-being between the settings, multivariate analysis of variance (MANOVA) was used because of theoretical concerns close relationship between subjective and psychological well-being - mentioned in 
Chapter One, and some recent empirical findings supporting the relationship between life satisfaction, trust, participation and health (Flavin, 2007; Mueller, 2009; Veenstra, 2002; H. M. Wang, Schlesinger, Wang, \& Hsiao, 2009; Yip, Subramanian, Mitchell, Lee, \& Wang, 2007). MANOVA reduces the chance of making Type I errors as it considers the correlation between the dependent variables (Field, 2005). A one way between subject MANOVA was performed on six dependent variables: life satisfaction, social trust, number of voluntary organizations joined, civic participations, and the two dimensions of SF-12: physical health and mental health. The independent variable was the setting: four churches and a social centre.

Both of the number of voluntary organizations joined and physical health were transformed due to the violation in the assumptions of normality. As mentioned before, an inverse transformation was used on the number of voluntary organizations joined. A reflect and square root transformation was used on the measure of SF12 - physical health. There were no univariate or multivariate outliers within cell at $\mathrm{p}<.001$. The results of evaluating assumptions of the homogeneity of variance-covariance matrix, linearity and multicollinearity were satisfactory.

Multivariate analysis demonstrated a significant overall effect between settings on the well-being outcomes (Wilk's $\Lambda=0.75, \mathrm{~F}(24,946.6)=3.40, \mathrm{p}<.001)$. Wilk's $\Lambda$ was significant, and the univariate comparisons can be interpreted without needing to adjust the individual alpha levels for each analysis.

To determine any significant differences regarding well-being between the five settings, univariate analyses were conducted, with post hoc tests. The results are summarized in Table 22. Life satisfaction was found to be significantly different across the five settings $\left(F(4,276)=4.30, p=.002, \eta^{2}=.059\right)$. Multiple comparisons using the Games-Howell procedure (very suitable for uneven samples, Field, 2005, p.341) indicated that the $\mathrm{CHC}$ members $(\mathrm{M}=24.55, \mathrm{SD}=6.04)$, $\mathrm{SCC}(\mathrm{M}=24.15, \mathrm{SD}=4.63)$, and $\mathrm{EVC}(\mathrm{M}=23.58, \mathrm{SD}=6.04)$ had significantly higher life satisfaction scores than those in the social centre $(M=20.25, S D=6.38)$. There was no significant difference between the four churches, and FDC was not significantly different from the social centre. 
Table 22 Means and Standard Deviations of well-being variables by members across settings

\begin{tabular}{|c|c|c|c|c|c|c|c|c|c|c|c|c|c|}
\hline & \multicolumn{2}{|c|}{ Social centre } & \multicolumn{2}{|c|}{$\mathrm{CHC}$} & \multicolumn{2}{|c|}{ SCC } & \multicolumn{2}{|c|}{ FDC } & \multicolumn{2}{|c|}{$\mathrm{EVC}$} & \multirow[b]{2}{*}{$\mathrm{F}$} & \multirow[b]{2}{*}{$\mathrm{p}$} & \multirow[b]{2}{*}{$\eta^{2}$} \\
\hline & M & $\mathrm{SD}$ & M & $\mathrm{SD}$ & M & $\mathrm{SD}$ & M & $\mathrm{SD}$ & M & $\mathrm{SD}$ & & & \\
\hline Satisfaction with Life & $20.25^{\mathrm{a}}$ & 6.38 & $24.55^{b}$ & 6.04 & $24.15^{b}$ & 4.63 & 22.47 & 6.14 & $23.58^{b}$ & 6.04 & 4.30 & .002 & .059 \\
\hline Social Trust & $28.14^{\mathrm{a}}$ & 4.10 & 29.87 & 4.42 & 29.41 & 3.56 & $31.09^{b}$ & 3.70 & 28.42 & 4.57 & 4.71 & .001 & .064 \\
\hline Civic participation & $12.12^{\mathrm{a}}$ & 2.34 & $11.94^{\mathrm{a}}$ & 2.71 & $13.61^{\mathrm{b}}$ & 2.70 & $11.53^{\mathrm{a}}$ & 2.65 & $12.06^{\mathrm{a}}$ & 2.78 & 3.96 & .004 & .054 \\
\hline $\begin{array}{l}\text { The number of voluntary } \\
\text { organization membership }\end{array}$ & $0.84^{\mathrm{a}}$ & 0.28 & $0.84^{\mathrm{a}}$ & 0.27 & $0.65^{\mathrm{b}}$ & 0.32 & $0.87^{\mathrm{a}}$ & 0.24 & $0.83^{\mathrm{a}}$ & 0.27 & 4.52 & .001 & .062 \\
\hline Physical health & 3.52 & 1.04 & 3.46 & 0.89 & 3.47 & 0.93 & 3.94 & 0.92 & 3.50 & 0.89 & 2.66 & .033 & .037 \\
\hline Mental health & $42.18^{\mathrm{a}}$ & 8.70 & 46.06 & 9.76 & $47.41^{\mathrm{b}}$ & 7.56 & 44.52 & 9.40 & 46.74 & 9.56 & 2.70 & .031 & .038 \\
\hline
\end{tabular}

Note. Means with different superscripts differ significantly at $\mathrm{p}<.05$ in the Games-Howell post hoc comparison 
Social trust was also found to be significantly different across the five settings $\left(\mathrm{F}(4,276)=4.71, \mathrm{p}=.001, \eta^{2}=.064\right)$. The Games-Howell post hoc test indicated that the social centre $(\mathrm{M}=28.14, \mathrm{SD}=4.10)$ and $\mathrm{EVC}(\mathrm{M}=28.42, \mathrm{SD}=4.57)$ had significantly lower scores than FDC $(\mathrm{M}=31.09, \mathrm{SD}=3.70)$. There were no significant differences between SCC and CHC and all other communities.

Civic participation was found to be significantly different across the five settings $\left(F(4,276)=3.96, p=.004, \eta^{2}=.054\right)$. The Games-Howell post hoc test indicated that the SCC members $(M=13.61, S D=2.70)$ have a significantly higher score regarding civic participation than those in the other settings, including the social centre $(\mathrm{M}=12.12, \mathrm{SD}=$ 2.34), and the other churches $\mathrm{CHC}(\mathrm{M}=11.94, \mathrm{SD}=2.71), \mathrm{EVC}(\mathrm{M}=12.06, \mathrm{SD}=2.78)$, FDC $(M=11.53, S D=2.65)$, while there was no significance difference between the social centre and the other three churches.

The number of voluntary organizations joined was also significantly different across the five settings $\left(\mathrm{F}(4,276)=4.52, \mathrm{p}=.001, \eta^{2}=.062\right)$. The Games-Howell post hoc test indicated that SCC members $(\mathrm{M}=.065, \mathrm{SD}=.32)$ have a significantly higher score $^{9}$ related to the number of voluntary organizations joined compared to those in the other settings, including the social centre $(\mathrm{M}=.84, \mathrm{SD}=.28)$ and the the other churches [CHC $(\mathrm{M}=.84, \mathrm{SD}=.27), \mathrm{EVC}(\mathrm{M}=.83, \mathrm{SD}=.27)$, FDC $(\mathrm{M}=.87, \mathrm{SD}=.24)]$. There was no significance difference between the social centre and the other three churches.

Physical health was found to be significantly different across the five settings ( $F(4$, 276) $=2.66, p=.033, \eta^{2}=.037$ ). The Games-Howell post hoc test indicated no significant differences between the mean of any setting.

The mental health (subscale of SF-12) was also significantly different across the five settings $\left(F(4,276)=2.70, p=.031, \eta^{2}=.038\right)$. The Games-Howell post hoc test indicated that SCC members $(\mathrm{M}=47.41, \mathrm{SD}=7.56)$ had a significantly higher mental health score than those in the social centre $(M=42.18, S D=8.70)$; regarding those in the other three churches, there was no difference in mental health score compared to both the social centre and SCC.

\footnotetext{
${ }^{9}$ As mentioned, as the variable was inversely transformed, the direction is reversed in interpretation.
} 


\subsubsection{Factorial multivariate analysis of variance}

A 2x5 between subjects multivariate analysis of variance was performed to further test for gender and setting effects. The same six dependent variables were used: life satisfaction, social trust, civic participation, mental health, transformation of physical health, and the number of affiliated organizations joined. The independent variables were the setting (the four churches and the social centre), and gender.

There were no univariate or multivariate outliers within cell at $\mathrm{p}<.001$. The results regarding assumptions of homogeneity of variance-covariance matrix, linearity and multicollinearity were satisfactory. Multivariate analysis indicated a significant effect between settings and well-being outcomes (Wilk's $\Lambda=0.74, \mathrm{~F}(24,915.2)=3.45$, p $<.001$ ), but not between gender and well-being outcomes (Wilk's $\Lambda=0.98, \mathrm{~F}(6,262)$ $=.84, \mathrm{p}=.54$, n.s.). Nevertheless, there is a significant interaction effect between gender and settings, on the well-being outcomes (Wilk's $\Lambda=0.86, \mathrm{~F}(24,915.2)=1.67, \mathrm{p}<.05$ ).

The impact of each main effect on individual DVs was explored and results summarized in Table 23. Univariate analyses indicated significant interaction effects between settings and gender on two well-being variables: satisfaction with life, F(4, $267)=2.71, p=.031, \eta^{2}=.039$ and social trust, $F(4,267)=3.86, p=.005, \eta^{2}=.055$. This indicates that males and females were affected differently by attending different spiritual settings related to life satisfaction and social trust. Specifically, there was no significant difference in the life satisfaction of males and female participants in the social centre and the three other churches, but the females $(M=25.49, S D=5.53)$ in the charismatic church have a significantly higher life satisfaction compared to males $(\mathrm{M}=22.8, \mathrm{SD}=6.54)$ (see Figure 5). Regarding social trust, a similar result accords with the charismatic church. While there was no significant difference in social trust between males and females participants in the social centre and the three other churches, this time males $(M=32.33$, $\mathrm{SD}=3.09$ ) in charismatic church have a significant higher social trust compared to their female counterparts $(\mathrm{M}=29.2, \mathrm{SD}=4.35)$. (see Figure 6). 
Table 23 Tests of the settings, sex and their interaction

\begin{tabular}{llllll}
\hline IV & DV & Univariate F & df & $\mathrm{p}$ & $\eta^{2}$ \\
\hline Setting & Life satisfaction & 4.068 & $4 / 267$ & .003 & .057 \\
& Social Trust & 5.875 & $4 / 267$ & .000 & .081 \\
& Civic participation & 3.870 & $4 / 267$ & .004 & .055 \\
& Voluntary organization & 4.550 & $4 / 267$ & .001 & .064 \\
& membership & 2.628 & $4 / 267$ & .035 & .038 \\
& Physical health & 3.219 & $4 / 267$ & .013 & .046 \\
& Mental health & & & & \\
& Lex & .008 & $1 / 267$ & .928 & .000 \\
& Life satisfaction & .398 & $1 / 267$ & .529 & .001 \\
& Social Trust & .412 & $1 / 267$ & .522 & .002 \\
& Civic participation & $1 / 267$ & .922 & .000 \\
& Voluntary organization & .010 & & .92 & \\
& membership & 3.217 & $1 / 267$ & .074 & .012 \\
& Physical health & .923 & $1 / 267$ & .338 & .003 \\
& Mental health & & & & \\
Setting by Sex & Life satisfaction & 2.706 & $4 / 267$ & .031 & .039 \\
& Social Trust & 3.855 & $4 / 267$ & .005 & .055 \\
& Civic participation & .372 & $4 / 267$ & .828 & .006 \\
& Voluntary organization & .984 & $4 / 267$ & .417 & .015 \\
& membership & & & & \\
& Physical health & .062 & $4 / 267$ & .993 & .001 \\
& Mental health & 2.244 & $4 / 267$ & .065 & .033 \\
\hline
\end{tabular}

Figure 5 Interaction of gender and setting in life satisfaction

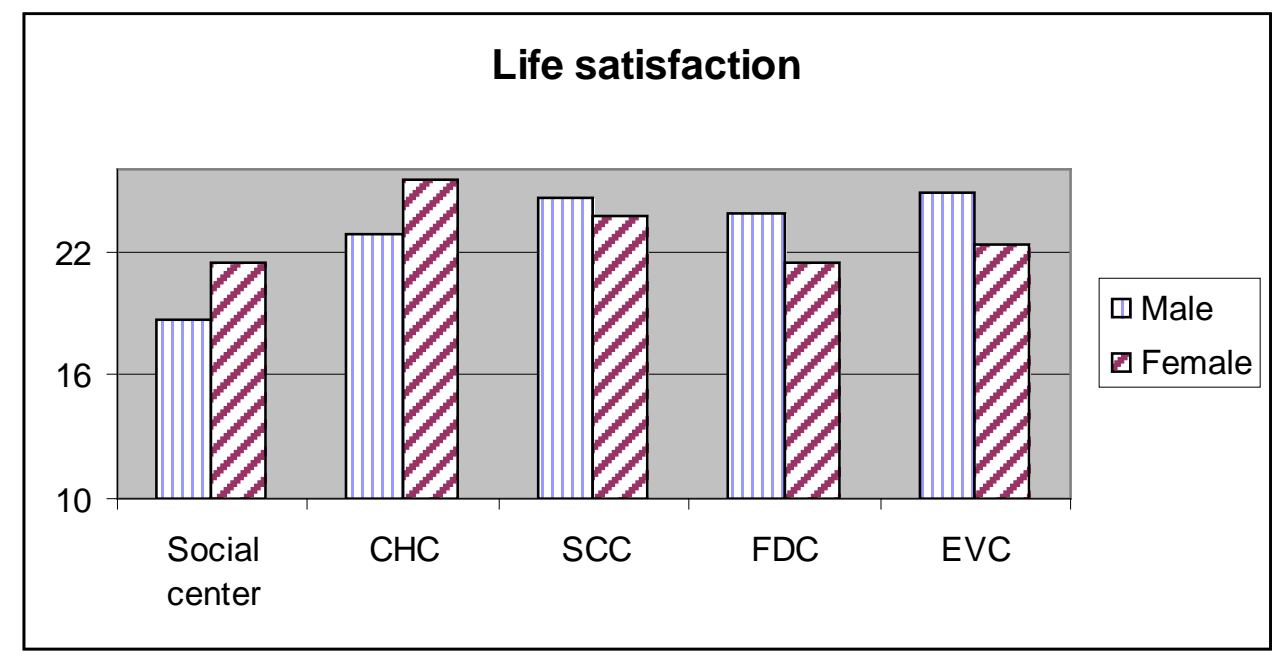


Figure 6 Interaction of gender and setting in social trust

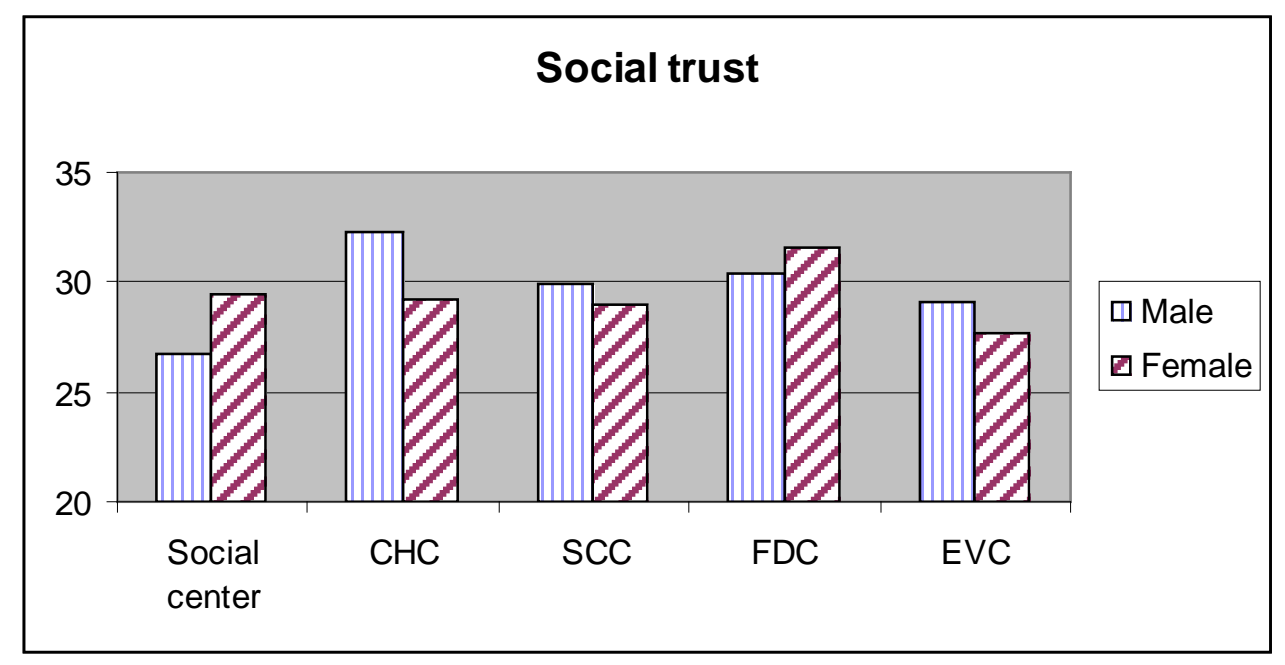

\subsubsection{Multivariate analysis of covariance}

As age is significantly different between the religious settings and the social centre, a 2 x 5 between subjects multivariate analysis of covariance was performed on the same six variables. The independent variables were the settings and gender, and age was used as a covariate.

Using Wilks' criterion, the combined DVs were significantly related to the age (the covariate), Wilk's $\Lambda=0.88, \mathrm{~F}(6,231)=5.49, \mathrm{p}<.001$, to setting Wilk's $\Lambda=0.76, \mathrm{~F}$ $(24,807.1)=2.78, p<.001$, and to the interaction, sex x setting, Wilk’s $\Lambda=0.85, F(24$, 807.1 $)=1.62, \mathrm{p}=.03$, but not to the $\operatorname{sex}(\mathrm{F}(6,231)=1.09, \mathrm{p}=0.37$, n.s. $)$.

The effects of the covariate and the setting and gender on the DVs after adjustment for covariates were investigated in univariate analysis and the results are summarized in Table 24. The covariate, age, was significantly related to participants' voluntary organizations joined, $F(1,236)=4.21, \mathrm{p}=.04, \eta^{2}=.018$; civic participation, (F $\left.(1,236)=7.55, p=.006, \eta^{2}=.031\right)$; physical health, $F(1,236)=5.79, p=.017, \eta^{2}=.024$; and mental health, $\mathrm{F}(1,236)=13.56, \mathrm{p}<.001, \eta^{2}=.054$. After controlling the covariates, ages, the effect of the setting was still significant on participants' life satisfaction, F (4, 236) $=2.60, \mathrm{p}=.037, \eta^{2}=.042$; social trust, $\mathrm{F}(4,236)=5.09, \mathrm{p}=.001, \eta^{2}=.079$; voluntary organizations joined, $\mathrm{F}(4,236)=4.40, \mathrm{p}=.002, \eta^{2}=.069$; civic participation, $\mathrm{F}(4$, 236)=3.52, $\mathrm{p}=.008<.05, \eta^{2}=.056$; physical health, $\mathrm{F}(4,236)=2.64, \mathrm{p}=.034, \eta^{2}=.043$; 
but not mental health, F $(4,236)=1.08$, p.367, n.s. The gender and setting interaction was still significant on participants' life satisfaction, $F(4,236)=2.79, p=.027, \eta^{2}=.045$;

social trust, $F(4,236)=3.93, p=.004, \eta^{2}=.062$.

Table 24 Tests of Age (covariate), the settings, sex and their interaction.

\begin{tabular}{llllll}
\hline IV & DV & Univariate F & df & $\mathrm{p}$ & $\eta^{2}$ \\
\hline Age(Covariate) & Life satisfaction & 2.374 & $1 / 236$ & .125 & .010 \\
& Social Trust & .180 & $1 / 236$ & .672 & .001 \\
& Civic participation & 4.211 & $1 / 236$ & .041 & .018 \\
& Voluntary organization & 7.549 & $1 / 236$ & .006 & .031 \\
& membership & 5.786 & $1 / 236$ & .017 & .024 \\
& Physical health & 13.557 & $1 / 236$ & .000 & .054 \\
& Mental health & 2.597 & $4 / 236$ & .037 & .042 \\
& Life satisfaction & 5.089 & $4 / 236$ & .001 & .079 \\
& Social Trust & 4.404 & $4 / 236$ & .002 & .069 \\
& Civic participation & & $4 / 236$ & .008 & .056 \\
& Voluntary organization & 3.518 & & & \\
& membership & 2.644 & $4 / 236$ & .034 & .043 \\
& Physical health & 1.080 & $4 / 236$ & .367 & .018 \\
& Mental health & .035 & $1 / 236$ & .852 & .000 \\
& Life satisfaction & .130 & $1 / 236$ & .719 & .001 \\
& Social Trust & .218 & $1 / 236$ & .641 & .001 \\
& Civic participation & $1 / 236$ & .120 & .010 \\
& Voluntary organization & 2.439 & & & \\
& membership & & $1 / 236$ & .149 & .009 \\
& Physical health & 2.095 & $1 / 236$ & .201 & .007 \\
& Mental health & 1.646 & $4 / 236$ & .027 & .045 \\
Setting by Sex & Life satisfaction & 2.789 & $4 / 236$ & .004 & .062 \\
& Social Trust & 3.933 & $4 / 236$ & .224 & .024 \\
& Civic participation & 1.434 & $4 / 236$ & .892 & .005 \\
& Voluntary organization & .278 & & & \\
& membership & .126 & $4 / 236$ & .973 & .002 \\
& Physical health & 1.661 & $4 / 236$ & .160 & .027 \\
\hline & Mental health & & & &
\end{tabular}

\subsubsection{Spirituality}

\subsubsection{One way Multivariate analysis of covariance}

Besides influencing well-being, it was expected that spiritual settings would also influence the participants' manifestations of spirituality. As age is different between various settings and the various dimensions of spirituality are closely related (discussed 
in Chapter Four), multivariate analysis of covariance was conducted to examine whether the four spirituality related variables vary as a function of the immediate settings after adjusting for differences in age. The four spirituality related variables are: fundamentalist belief, daily spiritual experience, time spent in church activities, and years attending church. When the independent variable was the setting, age was used as a covariate.

Both years of attending church and the time spent in church activities were transformed because of violations in the assumption of normality. As explained in the beginning of the chapter, a square root transformation was used on the measure of the years of attending church and a logarithmic transformation was used on the time spent in church activities.

There were six univariate outliers using a criterion $\mathrm{z}=|3.3|(\alpha=.001)$, in which two were also a multivariate outlier. All six outliers were deleted from the subsequent analyses. Although the evaluation of assumptions of linearity and multicollinearity were satisfactory, the assumption of homogeneity of variance-covariance matrix seems to be violated as the Box's $\mathrm{M}$ test is significant at $\mathrm{p}<.001$. As suggested by Tabachnick and Fidell (2007, p. 269) and Hair et al., (2006, p. 414), if sample sizes were unequal and the assumption of homogeneity of variance-covariance is violated, it is better to use Pillai's criterion instead of Wilks' lambda to evaluate the multivariate significance because of its robustness. Using Pillai's criterion, the combined DVs were significantly related to the age (the covariate), Pillai's $V=0.18, F(4,211)=11.77$, $p<.001$, to setting Pillai’s $V=0.86$, $\mathrm{F}(16,856)=14.64, \mathrm{p}<.001$.

The effects of the covariate and the setting on the DVs after adjustment for covariates were investigated in univariate analysis and the results are summarized in Table 25. The covariate was significantly related to the person's fundamentalist belief, $\mathrm{F}(1,214)=14.12, \mathrm{p}<.001, \eta^{2}=.062$, and time spent in church activities, $\mathrm{F}(1,214)=29.19$, $\mathrm{p}<.001, \eta^{2}=.12$. After controlling the covariates, the effect of the setting was still significant on participants' fundamentalist belief, $\mathrm{F}(4,214)=39.70, \mathrm{p}<.001, \eta^{2}=.426$; daily spiritual experience with God, $F(4,214)=19.40, \mathrm{p}<.001, \eta^{2}=.266$; year of attending church, $\mathrm{F}(4,214)=29.57, \mathrm{p}<.001, \eta^{2}=.356$; and time spent in church activities, $\mathrm{F}(4,214)=38.22, \mathrm{p}<.001, \eta^{2}=.417$. 
Planned contrasts (Table 26) reveal that the members of three churches, CHC $(\mathrm{M}=84.23, \mathrm{SD}=10.88)$, FDC $(\mathrm{M}=82.69, \mathrm{SD}=11.25)$, and $\mathrm{EVC}(\mathrm{M}=81.11, \mathrm{SD}=11.82)$, have a significantly higher score in fundamentalist belief compared to SCC members $(M=62.66, S D=10.40)$, and the social center $(M=56.95, S D=10.73)$.

In term of daily spiritual experience with God, the planned contrast also indicates that those in four churches had a significantly higher score in daily spiritual experience than those in social centre $(M=40.76, S D=17.88)$. $C H C$ members $(M=63.94, S D=12.14)$ and FDC (M= 61.36, SD=9.01) also have a significant higher score than SCC members $(\mathrm{M}=52.23, \mathrm{SD}=8.64)$ and $\mathrm{EVC}$ members $(\mathrm{M}=56.69, \mathrm{SD}=9.79)$ who have no significant difference in daily spiritual experience score with members of the other three churches.

Years of attending church was also found to be significantly different across the five settings. The contrast show that, as expected, members of the four churches have a significantly longer period attending the church than those in the social centre $(M=0.48$, $\mathrm{SD}=0.94)$. Within the church groups, $\mathrm{SCC}$ members $(\mathrm{M}=3.30, \mathrm{SD}=1.63$ and FDC $(\mathrm{M}=$ $3.56, \mathrm{SD}=1.13)$ have a significant higher score than $\mathrm{EVC}$ members $(\mathrm{M}=2.71, \mathrm{SD}=1.14)$. CHC members ( $M=2.81, S D=1.30)$ have no significant difference in daily spiritual experience score compared with SCC members, even though it is still significantly different from those in FDC.

Time spent in church activities was also found to be significantly different across the five settings. The contrast indicates that members of the four churches spend significantly longer time in church activities than those at the social centre $(M=0.48$, $\mathrm{SD}=0.94)$. Within the churches, $\mathrm{EVC}(\mathrm{M}=2.68, \mathrm{SD}=0.75)$, $\mathrm{CHC}(\mathrm{M}=2.81, \mathrm{SD}=1.30)$ and FDC members $(\mathrm{M}=2.77, \mathrm{SD}=0.63$ ) have a significantly higher scores than SCC members $(\mathrm{M}=1.88, \mathrm{SD}=1.12)$. 
Table 25 Tests of Age (covariate) and the settings

\begin{tabular}{llllll}
\hline IV & DV & Univariate F & df & p & $\eta^{2}$ \\
\hline Age(Covariate) & Fundamentalism & 14.12 & $1 / 214$ & .000 & .062 \\
& $\begin{array}{l}\text { Daily spiritual experience } \\
\text { with God }\end{array}$ & 1.16 & $1 / 214$ & .283 & .005 \\
& $\begin{array}{l}\text { Years of attending church } \\
\text { Time spent in church }\end{array}$ & 29.19 & $1 / 214$ & .000 & .120 \\
& activities & .000 & $1 / 214$ & .993 & .000 \\
Setting & Fundamentalism & 39.70 & $4 / 214$ & 000 & .426 \\
& $\begin{array}{l}\text { Daily spiritual experience } \\
\text { with God }\end{array}$ & 19.40 & $4 / 214$ & .000 & .266 \\
& $\begin{array}{l}\text { Years of attending church } \\
\text { Time spent in church }\end{array}$ & 29.57 & $4 / 214$ & .000 & .356 \\
& $\begin{array}{l}\text { activities } \\
\end{array}$ & 34.07 & $4 / 214$ & .000 & .417 \\
\hline
\end{tabular}

Table 26 Means and Standard Deviations of spirituality variables by members across settings

\begin{tabular}{lcccccccccc}
\hline & \multicolumn{2}{c}{ Social centre } & \multicolumn{2}{c}{ CHC } & \multicolumn{2}{c}{ SCC } & \multicolumn{2}{c}{ FDC } & \multicolumn{2}{c}{ EVC } \\
& M & SD & M & SD & M & SD & M & SD & M & SD \\
\hline $\begin{array}{l}\text { Fundamentalism } \\
\text { belief }\end{array}$ & $56.95^{\mathrm{a}}$ & 10.73 & $84.23^{\mathrm{D}}$ & 10.88 & $62.66^{\mathrm{a}}$ & 10.40 & $82.69^{\mathrm{D}}$ & 11.25 & $81.11^{\mathrm{D}}$ & 11.82 \\
$\begin{array}{l}\text { Daily } \\
\text { spirituality } \\
\text { experience }\end{array}$ & $40.76^{\mathrm{a}}$ & 17.88 & $63.94^{\mathrm{c}}$ & 12.14 & $52.23^{\mathrm{D}}$ & 8.64 & $61.36^{\mathrm{c}}$ & 9.01 & $59.69^{\mathrm{Dc}}$ & 9.79 \\
$\begin{array}{l}\text { Years of } \\
\text { attending } \\
\text { church }\end{array}$ & $0.48^{\mathrm{a}}$ & 0.94 & $2.81^{\mathrm{D}, \mathrm{c}}$ & 1.30 & $3.30^{\mathrm{c}, \mathrm{a}}$ & 1.63 & $3.56^{\mathrm{a}}$ & 1.13 & $2.71^{\mathrm{D}}$ & 1.14 \\
$\begin{array}{l}\text { Time spent in } \\
\text { church activities }\end{array}$ & $0.48^{\mathrm{a}}$ & 0.94 & $2.81^{\mathrm{c}}$ & 1.30 & $1.88^{\mathrm{D}}$ & 1.12 & $2.77^{\mathrm{c}}$ & 0.63 & $2.68^{\mathrm{c}}$ & 0.75 \\
\hline
\end{tabular}

Note. Means with different superscripts differ significantly at $\mathrm{p}<.05$ in the contrast analysis

\subsubsection{Sense of community and social belief}

\subsubsection{One way Multivariate analysis of variance}

It was proposed that spiritual settings may influence participants' feelings of sense of community and certain social beliefs. As age is a potential covariate, a series of ANCOVAs were conducted to ascertain differences in the participants' sense of community within their immediate setting and the five social beliefs: social cynicism, social flexibility, reward for application, fate control, and religiosity. 
The six variables did not exhibit a serious violation of normality assumption and the results of evaluating assumptions of homogeneity of variance-covariance matrix, linearity and multicollinearity were satisfactory. Nevertheless, there was three univariate outliers using a criterion $\mathrm{z}=|3.3|(\alpha=.001)$, in which one was also one of the two multivariate outliers ( $\alpha=.001)$. All four outliers were deleted from the analyses.

The multivariate analysis demonstrated that the combined DVs were significantly related to the age (the covariate), Wilk's $\Lambda=0.536$, F $(34,967.5)=7.90, \mathrm{p}<.001$. In addition, there are also significant overall effects between settings on the outcomes (Wilk’s $\Lambda=0.536, \mathrm{~F}(34,967.5)=7.90, \mathrm{p}<.001)$. As Wilk’s $\Lambda$ was significant, the univariate comparisons can be interpreted without needing to adjust individual alpha levels for each analysis.

The effects of the covariate and the setting on the DV after adjustment for covariates were investigated in univariate analysis and the results are summarized in Table 27. The covariate was significantly related to the participants' social cynicism $\mathrm{F}(1$, $241)=4.64, \mathrm{p}=.032)$, reward for application, $\mathrm{F}(1,241)=4.47, \mathrm{p}=0.036$, and religiosity, $\mathrm{F}(1,241)=5.91, \mathrm{p}=0.016)$. After controlling the covariates, ages, the effect of the setting was still significant on participants' sense of community, $F(4,241)=15.49, \mathrm{p}<.001, \eta^{2}=$ 0.205, reward for application, $\mathrm{F}(4,241)=2.71, \mathrm{p}=0.031, \eta^{2}=0.043$, fate control, $\mathrm{F}(4$, $241)=9.49, \mathrm{p}<.001, \eta^{2}=0.136$, and religiosity, $\mathrm{F}(4,241)=13.42, \mathrm{p}<.001, \eta^{2}=0.182$

Planned contrasts (Table 28) reveal that there are significant differences between the settings for sense of community, fate control and religiosity. Members in two churches CHC (M=43.26, $\mathrm{SD}=4.47)$, FDC $(\mathrm{M}=42.59, \mathrm{SD}=4.10)$ have a significantly higher score in sense of community than SCC $(M=38.91, \mathrm{SD}=4.30)$, EVC members $\mathrm{M}=$ 39.36, $\mathrm{SD}=4.43)$ and those at the social centre $(\mathrm{M}=36.43, \mathrm{SD}=5.01)$.

Fate control was also found to be significantly different across the five settings. Those at the social centre $(\mathrm{M}=14.85, \mathrm{SD}=2.64)$ had significantly higher fate control than the three churches [FDC $(\mathrm{M}=11.46, \mathrm{SD}=2.87)$, EVC $(\mathrm{M}=11.46, \mathrm{SD}=3.09), \mathrm{CHC}(\mathrm{M}=$ 12.19, $\mathrm{SD}=2.65)]$ but not SCC $(\mathrm{M}=13.52, \mathrm{SD}=2.34)$. While SCC members have no significant difference in the fate control score compared with those at the social centre and charismatic church, CHC members have no significant difference in the score with those in other churches. 
Table 27 Tests of Age (covariate) and the settings

\begin{tabular}{llllll}
\hline IV & DV & Univariate F & df & $\mathrm{p}$ & $\eta^{2}$ \\
\hline Age(Covariate) & Sense of community & .505 & $1 / 241$ & .478 & .002 \\
& Social Cynicism & 4.64 & $1 / 241$ & .032 & .019 \\
& Reward for & 4.47 & $1 / 241$ & .036 & .018 \\
& application & .481 & $1 / 241$ & .489 & .002 \\
& Social flexibility & .238 & $1 / 241$ & .626 & .001 \\
& Fate control & 5.91 & $1 / 241$ & .016 & .024 \\
& Religiosity & & & & \\
& Setting & 15.49 & $4 / 241$ & .000 & .205 \\
& Social Cynicism & 1.00 & $4 / 241$ & .407 & .016 \\
& Reward for & 2.71 & $4 / 241$ & .031 & .043 \\
& application & 1.29 & $4 / 241$ & .276 & .021 \\
& Social flexibility & 1.49 & $4 / 241$ & .000 & .136 \\
& Fate control & 9.49 & $4 / 241$ & .000 & .182 \\
\hline & Religiosity & 13.42 & & &
\end{tabular}

Table 28 Means and Standard Deviations of sense of community and social belief by members across settings

\begin{tabular}{|c|c|c|c|c|c|c|c|c|c|c|}
\hline & \multicolumn{2}{|c|}{ Social centre } & \multicolumn{2}{|c|}{ CHC } & \multicolumn{2}{|c|}{ SCC } & \multicolumn{2}{|c|}{ FDC } & \multicolumn{2}{|c|}{ EVC } \\
\hline & M & SD & M & SD & M & SD & M & SD & M & SD \\
\hline $\begin{array}{l}\text { Sense of } \\
\text { community }\end{array}$ & $36.43^{\mathrm{a}}$ & 5.01 & $43.26^{0}$ & 4.47 & $38.91^{\mathrm{a}}$ & 4.30 & $42.59^{\circ}$ & 4.10 & $39.36^{a}$ & 4.43 \\
\hline $\begin{array}{l}\text { Social } \\
\text { Cynicism }\end{array}$ & 17.03 & 2.75 & 16.32 & 3.51 & 16.63 & 3.03 & 16.57 & 3.00 & 16.50 & 2.85 \\
\hline $\begin{array}{l}\text { Reward for } \\
\text { Application }\end{array}$ & 17.83 & 2.85 & 19.32 & 2.61 & 18.31 & 2.67 & 17.73 & 2.32 & 18.04 & 2.75 \\
\hline $\begin{array}{l}\text { Social } \\
\text { Flexibility }\end{array}$ & 19.28 & 2.08 & 19.15 & 2.60 & 18.83 & 2.58 & 19.57 & 2.13 & 19.86 & 2.21 \\
\hline $\begin{array}{l}\text { Fate } \\
\text { control }\end{array}$ & $14.85^{\mathrm{a}}$ & 2.64 & $12.19^{\mathrm{D}, \mathrm{c}}$ & 2.65 & $13.51^{\mathrm{a}, \mathrm{D}}$ & 3.48 & $11.46^{\mathrm{c}}$ & 2.87 & $11.46^{\mathrm{C}}$ & 3.09 \\
\hline Religiosity & $17.00^{\mathrm{a}}$ & 3.25 & $21.09^{c}$ & 2.55 & $19.11^{\mathrm{ab}}$ & 2.34 & $20.12^{\mathrm{DC}}$ & 2.61 & $21.30^{\mathrm{c}}$ & 2.07 \\
\hline
\end{tabular}

Note. Means with different superscripts differ significantly at $\mathrm{p}<.05$ in the contrast analysis

Religiosity was found to be significantly different across the five settings. The planned contrast indicates that those at the social centre $(\mathrm{M}=17.00, \mathrm{SD}=3.25)$ had a significantly lower spirituality than three churches [FDC $(\mathrm{M}=20.12, \mathrm{SD}=2.61)$, $\mathrm{EVC}(\mathrm{M}=21.30, \mathrm{SD}=$ 2.07), $\mathrm{CHC}(\mathrm{M}=21.09, \mathrm{SD}=2.55)]$ but not SCC $(\mathrm{M}=19.11, \mathrm{SD}=2.34)$ While SCC members have no significant difference in the religiosity with those in the social centre and fundamentalist church, the FDC members have no significant difference in the score with members of other churches. 


\subsection{Underlying patterns between groups}

\subsubsection{Discriminant Analysis}

To further understand the significant multivariate finding, descriptive discriminant analysis (DDA) was conducted to explore how the various settings (both religious and non-religious) differ in the manifestation of well-being, spirituality and context related variables. Instead of giving the pattern of difference on each individual dependent variable between the groups, DDA provided information about the underlying dimensions (or latent variables) along which groups differ (Huberty, 1994; Huberty \& Hussein, 2003; Huberty, Olejnik, \& Huberty, 2006; Tabachnick \& Fidell, 2007). That is, it shows how many dimensions characterize groups separately, and indicates underlying constructs or patterns characterizing group separation.

The 16 outcome variables were: life satisfaction; social trust; civic participation; mental health and (transformation of) physical health; (transformation of) membership of voluntary organizations; fundamentalist belief; daily spiritual experience; and (transformation of) years of attending church; (transformation of) time spent in church activities; sense of community; social cynicism; social flexibility; reward for application; fate control; and religiosity. The grouping variable was the settings.

Because of DDA's sensitivity to outliers, 9 univariate outliers were identified and deleted from subsequent analyses. A total of 324 participants were reduced to 209 after the deletion of cases missing at least one outcome variable. For the remaining 209 cases (40, 38, 34, 38 and 59 members respectively from social centre, CHC, SCC, FDC, and EVC), the results of evaluation of assumptions of linearity and multicollinearity were satisfactory. Observation of matrices indicated homogeneity of covariance was violated; however, this did not have an impact on the results.

The data were analysed using SPSS version 15.0 and four significant discriminant functions were revealed. The first function explained $71.1 \%$ of the variance, canonical $\mathrm{R}^{2}$ $=0.70$; the second function explained $14.4 \%$, canonical $\mathrm{R}^{2}=0.32$; the third function explained $10.3 \%$, canonical $R^{2}=0.25$; the fourth function explained $4.2 \%$, canonical $R^{2}=$ 0.12 . Wilks' lambda was statistically significant for the four combined functions, $\Lambda=.136$, $\left.\chi^{2}(64)=393.94, \mathrm{p}<.001\right)$, three combined functions when the first function was removed, 
$\Lambda=.45, \chi^{2}(45)=157.733, \mathrm{p}<.001$, two combined function when the first two functions were removed $\Lambda=.661, \chi^{2}(28)=81.835, \mathrm{p}<.001$, and the last function when the first three functions were removed $\Lambda=.881, \chi^{2}(13)=25.108, \mathrm{p}<.05$. Table 29 presents these findings.

Table 29 Wilks's lambda and Canonical Correlation for the five settings

\begin{tabular}{lllllll}
\hline Test of & Wilks' & & & & & \\
Function(s) & Lambda & Chi-square & Df & Sig. & $\mathrm{R}_{\mathrm{c}}$ & $\mathrm{R}_{\mathrm{c}}{ }^{2}$ \\
\hline 1 through 4 & .136 & 393.940 & 64 & .000 & .835 & .70 \\
2 through 4 & .450 & 157.733 & 45 & .000 & .565 & .32 \\
3 through 4 & .661 & 81.835 & 28 & .000 & .500 & .25 \\
4 & .881 & 25.108 & 13 & .022 & .346 & .12 \\
\hline
\end{tabular}

$\mathrm{R}_{\mathrm{c}}$ is canonical correlation

Structures were examined to assess the correlations between each outcome variable and the linear discriminant function (LDF). Table 30 shows that the first functions is defined primarily by the spirituality-related variables (fundamentalist belief, time spent in church activities, daily spiritual experience, religiosity and fate control). The second construct was primarily defined by a mixture of well-being and spiritualityrelated variables (years attending church, number of voluntary organizations joined, civic participation, and mental health). The third construct was defined primarily by sense of community and social flexibility. The last construct was defined by a mixture of wellbeing variables and social beliefs (reward for application, physical health, social trust, life satisfaction and social cynicism).

Group centroids were further examined (Table 31) and a LDF plot of group centroids is given in Figure 7. From the Table 31 and the LDF plot, it appears that LDF1 separates the social centre from the church groups. While the LDF2 separates the SCC from the other religious groups, LDF3 separates EVC from two churches (FDC and CHC). The last LDF4, despite not having a very explicit effect, separates CHC from FDC. 
Table 30 Standardized discriminant function and structure coefficients for the five settings

\begin{tabular}{lcccc}
\hline & \multicolumn{4}{c}{ Function } \\
& 1 & 2 & 3 & 4 \\
\hline Fundamentalism & $.661\left(^{*}\right)$ & -.270 & -.266 & .120 \\
Time spent in church & $.639\left(^{*}\right)$ & .217 & -.042 & -.292 \\
activities & & & & \\
Daily spiritual experience & $.470\left(^{*}\right)$ & .021 & .126 & -.232 \\
with God & $.454\left(^{*}\right)$ & .052 & -.252 & -.281 \\
Religiosity & $-.298\left(^{*}\right)$ & -.064 & .264 & -.050 \\
Fate Control & & & & \\
& .467 & $.783\left(^{*}\right)$ & .178 & .094 \\
Years of attending church & .039 & $-.351\left(^{*}\right)$ & -.020 & .218 \\
Affiliated voluntary & & $.261\left(^{*}\right)$ & -.008 & -.247 \\
organization membership &. .017 & $.202\left(^{*}\right)$ & -.090 & -.140 \\
Civic participation & .080 & & & \\
Mental health & & -.118 & $.647\left(^{*}\right)$ & -.098 \\
Sense of community & .387 & -.030 & $-.304\left(^{*}\right)$ & .183 \\
Social Flexibility & .033 & & & \\
& & -.035 & .301 & $-.489\left(^{*}\right)$ \\
Reward for Application & .032 & .079 & .091 & $.403\left(^{*}\right)$ \\
Physical health & .084 & .017 & .186 & $.290\left(^{*}\right)$ \\
Social Trust & .132 & .134 & .072 & $-.253\left(^{*}\right)$ \\
Life satisfaction & .102 & .048 & -.079 & $.195\left(^{*}\right)$ \\
Social Cynicism & -.032 & &
\end{tabular}

Table 31 Group centroids

\begin{tabular}{lcccc}
\hline & \multicolumn{4}{c}{ Function } \\
Church & 1 & 2 & 3 & 4 \\
\hline Secular & -2.728 & -.578 & -.013 & .159 \\
Charismatic & 1.089 & -.710 & .645 & -.459 \\
Social justice & -.809 & 1.361 & .400 & -.204 \\
Fundamentalism & 1.423 & .040 & .330 & .657 \\
evangelical & .698 & .038 & -.849 & -.117 \\
\hline
\end{tabular}




\section{Canonical Discriminant Functions}

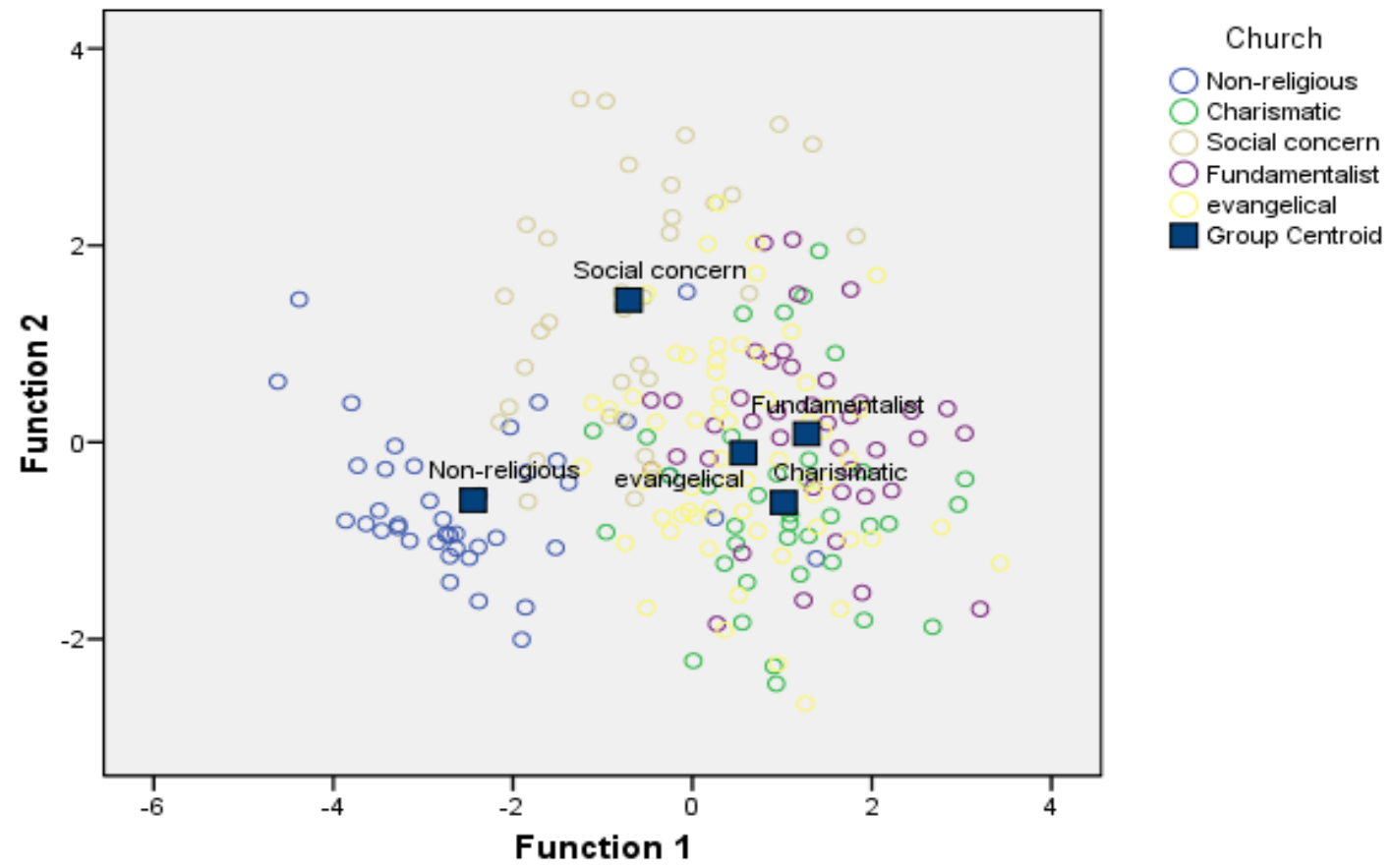

Figure 7: LDF Plot

To be more specific, the separation of social centre from the other church groups can be attributed to spirituality related variables. The separation of SCC church from the other church group may be attributed to the years of attending church and civic participation. The separation of EVC from the other two churches (CHC and FDC) is mainly attributed to the sense of community and social flexibility. The differentiation between CHC and FDC may be related to the set of well-being variables.

\subsection{The influence of spirituality}

After exploring the influence of spiritual context on well-being, spirituality and contextual variables, in the following section we explore whether spirituality variables are associated with well-being outcome and determine whether spirituality variables can predict well-being scores, even while holding constant other major predictors. The primarily focus is on three well-being outcomes: life satisfaction, social trust, and civic participation. 


\subsubsection{Relationship between Spirituality and life satisfaction}

Pearson's correlation of the key variables was conducted and the findings are displayed in Table 32. It is shown that physical health, mental health, social cynicism, reward for application, religiosity (social belief), fundamentalism, daily spiritual experience (DSES), sense of community, years attending church, time spending in church activities, and age, were significantly positively correlated with satisfaction with life. With the exception of the two dependent variables (social trust and civic participation), the other variables were then entered into a series of regression analyses.

\subsubsection{Prediction of spirituality related variables on life satisfaction}

In the first regression analysis, we examine whether spirituality related variables are associated with life satisfaction. The assumptions of regression analyses were examined. As the years of attending church and the time spent in church activities were skewed and with univariate outliers, transformations were applied. As stated previously, square root transformation was applied to the years of attending church and log transformation was applied to the time spent in church activities. After transformation, no univariate outlier was found; however, three other cases were identified through Mahalanobis distance as multivariate outliers with $\mathrm{p}<.001$ and were deleted from subsequent analyses.

The four spirituality-related variables (fundamentalism, daily spiritual experience, years of attending church, and time spent in church activities) were entered into regression analysis as predictor variables, using the stepwise method because of its exploratory nature (Tabachnick \& Fidell, 2007). The results indicate that two spirituality related variables (time spent in church activities and daily spiritual experience) were identified as significant predictors of satisfaction with life (see Table 33). While daily spiritual experience was entered into the equation first, time spent in the church activities followed. The results were significant, $\mathrm{F}(2,251)=14.15, \mathrm{p}<.001$, and the adjusted $\mathrm{R}^{2}$ indicated that nearly $10 \%$ of the variance in life satisfaction is explained by the two spirituality-related variables. 
Table 32 Inter-correlations between all key variables

\begin{tabular}{|c|c|c|c|c|c|c|c|c|c|c|c|c|c|c|c|c|c|}
\hline \multicolumn{2}{|c|}{ Variable } & 1 & 2 & 3 & 4 & 5 & 6 & 7 & 8 & 9 & 10 & 11 & 12 & 13 & 14 & 15 & 16 \\
\hline \multirow{3}{*}{$\begin{array}{l}1 \\
2 \\
3\end{array}$} & Life satisfaction & & & & & & & & & & & & & & & & \\
\hline & Social Trust & $.335(* *)$ & & & & & & & & & & & & & & & \\
\hline & $\begin{array}{l}\text { Civic } \\
\text { participation }\end{array}$ & $.119\left(^{*}\right)$ & $.131\left(^{*}\right)$ & & & & & & & & & & & & & & \\
\hline 4 & $\begin{array}{l}\text { Affiliated } \\
\text { voluntary } \\
\text { organization } \\
\text { membership }\end{array}$ & 0.028 & -0.011 & $.450(* *)$ & & & & & & & & & & & & & \\
\hline 5 & Physical health & $\begin{array}{l}- \\
.219(* *)\end{array}$ & $-.116\left(^{*}\right)$ & 0.015 & -0.066 & & & & & & & & & & & & \\
\hline \multirow{2}{*}{$\begin{array}{l}6 \\
7\end{array}$} & Mental health & $.440(* *)$ & $.296(* *)$ & 0.046 & -0.014 & -0.066 & & & & & & & & & & & \\
\hline & $\begin{array}{l}\text { Sense of } \\
\text { community }\end{array}$ & $.335(* *)$ & $.292(* *)$ & $.160(* *)$ & -0.018 & 0.075 & $.213(* *)$ & & & & & & & & & & \\
\hline 8 & Social Cynicism & $-.124(*)$ & $\begin{array}{l}- \\
.260(* *)\end{array}$ & -0.010 & 0.011 & 0.056 & -0.078 & -0.098 & & & & & & & & & \\
\hline 9 & $\begin{array}{l}\text { Reward for } \\
\text { Application }\end{array}$ & $.120(*)$ & 0.090 & $.118\left(^{*}\right)$ & -0.105 & -0.019 & 0.098 & $.146(*)$ & 0.103 & & & & & & & & \\
\hline \multirow{2}{*}{$\begin{array}{l}10 \\
11\end{array}$} & Social Flexibility & -0.082 & -0.027 & 0.078 & -0.035 & -0.083 & 0.073 & -0.059 & $.316(* *)$ & 0.105 & & & & & & & \\
\hline & Fate control & -0.091 & $\begin{array}{l}- \\
.176(* *)\end{array}$ & 0.030 & -0.033 & -0.014 & $-.141(*)$ & $\begin{array}{l}- \\
.221(* *)\end{array}$ & $.230(* *)$ & 0.046 & -0.016 & & & & & & \\
\hline 12 & $\begin{array}{l}\text { Religiosity (social } \\
\text { belief ) }\end{array}$ & $.253(* *)$ & $.166(* *)$ & $.169(* *)$ & 0.000 & 0.048 & $.191(* *)$ & $.362(* *)$ & 0.016 & $.292(* *)$ & $.238(* *)$ & $\begin{array}{l}- \\
.401(* *)\end{array}$ & & & & & \\
\hline 13 & Fundamentalism & $.231(* *)$ & $.168(* *)$ & -0.002 & 0.095 & 0.041 & $.215\left(^{* *}\right)$ & $.436(* *)$ & 0.003 & 0.010 & 0.114 & $.471(* *)$ & $.571(* *)$ & & & & \\
\hline 14 & $\begin{array}{l}\text { Daily spiritual } \\
\text { experience }\end{array}$ & $.283(* *)$ & $.142(*)$ & $.140(*)$ & -0.031 & 0.053 & $.172(* *)$ & $.504(* *)$ & $-.138\left(^{*}\right)$ & 0.064 & -0.060 & $.294(* *)$ & $.471(* *)$ & $.496(* *)$ & & & \\
\hline 15 & $\begin{array}{l}\text { Years of attending } \\
\text { church }\end{array}$ & $.215(* *)$ & $.188(* *)$ & $.181(* *)$ & $-.125\left(^{*}\right)$ & $.125\left(^{*}\right)$ & $.219(* *)$ & $.372(* *)$ & 0.021 & 0.045 & 0.057 & $.263(* *)$ & $.367(* *)$ & $.353(* *)$ & $.417\left({ }^{* *}\right)$ & & \\
\hline \multirow{2}{*}{$\begin{array}{l}16 \\
17\end{array}$} & $\begin{array}{l}\text { Time spent in } \\
\text { church activities }\end{array}$ & $.274(* *)$ & $.129(*)$ & $.169(* *)$ & -0.026 & 0.081 & $.181(* *)$ & $.441(* *)$ & -0.015 & 0.007 & -0.025 & $.325(* *)$ & $.498(* *)$ & $.519(* *)$ & $.547(* *)$ & $.545(* *)$ & \\
\hline & Age & $.175(* *)$ & -0.002 & -0.057 & 0.096 & $.199(* *)$ & $.277(* *)$ & $.174(* *)$ & 0.066 & 0.096 & 0.037 & $.157(* *)$ & $.338(* *)$ & $.353(* *)$ & $.308(* *)$ & $.475(* *)$ & $.295(* *)$ \\
\hline
\end{tabular}


Table 33 Stepwise Regression of Spirituality related variables on Life Satisfaction

\begin{tabular}{llllll}
\hline Variable & $\mathrm{B}$ & $\mathrm{SEB}$ & $\mathrm{Beta}$ & $\mathrm{R}^{2}$ & Adjusted R $^{2}$ \\
\hline $\begin{array}{l}\text { Daily spiritual } \\
\text { experience with God }\end{array}$ & .074 & .031 & $.179 *$ & .080 & .077 \\
$\begin{array}{l}\text { Time spent in church } \\
\text { activities }\end{array}$ & .833 & .345 & $.179 *$ & .101 & .094 \\
\hline $\mathrm{p}<.05, * * \mathrm{p}<.01$ & & & & &
\end{tabular}

\subsubsection{Prediction of spirituality related variables on sense of community}

The second regression analysis examined if the variance of sense of community can be explained by spirituality related variables. Pearson's product correlation indicated that sense of community was correlated with all the spirituality related variables: fundamentalism, daily spiritual experience, years attending church, and time spent in church activities, and all four variables were entered into the regression equation using the stepwise method. Two multivariate outliers were identified and deleted. Table 34 shows that daily spiritual experience, fundamentalist belief and years attending church are identified as significant predictors of sense of community. Daily spiritual experience was the first predictor entered into the equation, and fundamentalist belief and years attending church were entered subsequently. The result was significant, $\mathrm{F}(3,244)=$ 42.15, $\mathrm{p}<.001$, and the adjusted $\mathrm{R}^{2}$ indicated that one third (33.3\%) of the variance of sense of community can be explained by spirituality related variables: a combination of fundamentalism, daily spiritual experience, and years attending church.

Table 34 Stepwise Regression of Spirituality related variables on Sense of community

\begin{tabular}{llllll}
\hline Variable & $\mathrm{B}$ & $\mathrm{SEB}$ & $\mathrm{Beta}$ & $\mathrm{R}^{2}$ & Adjusted R $^{2}$ \\
\hline $\begin{array}{l}\text { Daily spiritual } \\
\text { experience with God }\end{array}$ & .113 & .023 & $.328^{* * *}$ & .279 & .276 \\
$\begin{array}{l}\text { Fundamentalism } \\
\begin{array}{l}\text { Years of attending } \\
\text { church }\end{array}\end{array}$ & .069 & .021 & $.209 * *$ & .318 & .312 \\
\hline Note: ${ }^{*} \mathrm{p}<.05, * * \mathrm{p}<.01,{ }^{* * *} \mathrm{p}<.001$ & .193 & $.176^{* *}$ & .341 & .333 \\
\end{tabular}




\subsubsection{Prediction of spirituality related variable on mental health}

A third regression analysis examined if spirituality related variables help explain variations in mental health. As with the sense of community variable, Pearson's product correlation also showed that mental health was correlated with all the spirituality related variables: fundamentalism, daily spiritual experience, years attending church, and time spent in church activities. One univariate outlier and two multivariate outliers were identified and deleted from analysis.

Four spirituality-related variables were entered into regression analysis as predictor variables, using the stepwise method. Table 35 shows that fundamentalist belief and years of attending churches were identified as significant predictors of mental health. Fundamentalist belief was entered into the equation first, and years of attending church was entered later. Although the result is significant, F (2, 247) $=7.47, \mathrm{p}<.005$, the adjusted $\mathrm{R}^{2}$ indicated that only about $5 \%$ of the variance of mental health can be explained by spirituality related variables: a combination of fundamentalism and years attending churches.

Table 35: Stepwise regression of spirituality related variables on mental health

\begin{tabular}{llllll}
\hline Variable & $\mathrm{B}$ & $\mathrm{SEB}$ & Beta & $\mathrm{R}^{2}$ & Adjusted R $^{2}$ \\
\hline Fundamentalism & .090 & .040 & $.148^{*}$ & .039 & .036 \\
$\begin{array}{l}\text { Years of attending } \\
\text { church }\end{array}$ & .819 & .382 & $.142^{*}$ & .057 & .049 \\
\hline
\end{tabular}

Note: ${ }^{*} \mathrm{p}<.05,{ }^{* *} \mathrm{p}<.01,{ }^{* * *} \mathrm{p}<.001$

\subsubsection{Prediction of life satisfaction}

In the fourth regression analysis, all the variables correlated with life satisfaction shown in the correlation table were entered into the equation, using three blocks. As well as years attending church and the time spent in church activities, physical health was also transformed because of the violation of the normality assumption and the presence of univariate outliers. Reflect and square root transformation was applied to physical health. Four cases with extremely high z scores respectively on transformed physical health, mental health and religiosity variables were found to be univariate outliers. Two other cases were identified through Mahalanobis distance as multivariate outliers with $\mathrm{p}<.001$. All six outliers were deleted from the analyses. 
Table 36 shows that physical health, mental health and sense of community were significant predictors of life satisfaction. As mental health was the first predictor entered into the equation, physical health and sense of community followed in order. The result is significant, $\mathrm{F}(3,194)=32.91, \mathrm{p}<.001$, and the adjusted $\mathrm{R}^{2}$ indicates that about one third $(32.7 \%)$ of the variance of life satisfaction can be explained by the three predictors. It is worth noting that since physical health has been "reflect and square root" transformed, the negative $\beta$ coefficient shows a positive association between physical health and life satisfaction. In addition, despite predictions, no spirituality-related variable was shown to significantly contribute to the prediction of life satisfaction once the stated variables are taken into account.

Table 36: Stepwise regression of variables predicting life satisfaction

\begin{tabular}{llllll}
\hline Variable & $\mathrm{B}$ & $\mathrm{SEB}$ & Beta & $\mathrm{R}^{2}$ & Adjusted ${ }^{2}$ \\
\hline Mental health & .281 & .041 & $.409^{* * *}$ & .232 & .228 \\
Physical health & -1.606 & .371 & $-.255^{* * *}$ & .292 & .285 \\
Sense of community & .262 & .072 & $.217^{* * *}$ & .337 & .327 \\
\hline Note: ${ }^{*} \mathrm{p}<.05,{ }^{* *} \mathrm{p}<.01,{ }^{* * *} \mathrm{p}<.001$ & & & &
\end{tabular}

\subsubsection{Summary}

Although the correlation table and the first regression equation show that all the spirituality-related variables were significantly correlated with and predictive of life satisfaction, the fourth regression analysis suggests that the effect of these spirituality related variables was cancelled out when physical health, mental health, and sense of community were taken into consideration. Nevertheless, the second and third regression analyses equations reveal that spirituality-related variables significantly affect sense of community and mental health. Fulfilling the four conditions mentioned by Baron and Kenny (1986) and MacKinnon, Fairchild, and Fritz (2007), the present study seems to suggest that the relationship between spirituality related variables and life satisfaction is mediated through mental health and sense of community.

\subsubsection{Relation between Spirituality and Social Trust}

Pearson's product correlation (Table 32) indicates that social trust was significantly correlated with the following 10 variables: physical health, mental health, 
social cynicism, fate control, religiosity (social belief), fundamentalism, daily spiritual experience, sense of community (SCI), years of attending church, and time spent in church activities. Two separate regression analyses were conducted to examine the effects of spirituality-related variables on social trust and its effect when the other variables are controlled.

\subsubsection{Prediction of spirituality related variables on social trust}

In the first regression analysis, the association between spirituality related variables and social trust was examined. As previously, years of attending church and time spent in church activities were transformed accordingly. While one univariate outlier was found, two other cases were identified through Mahalanobis distance as multivariate outliers with $\mathrm{p}<.001$. All three outliers were deleted from the subsequent analysis.

The four spirituality-related variables (fundamentalism, daily spiritual experience, years attending church, and time spent in church activities) were entered into regression analysis as predictor variables, using the stepwise method because of its exploratory nature (Tabachnick \& Fidell, 2007). The results (in Table 37) indicate that only one spirituality related variable - daily spiritual experience -- was identified as a significant predictor of social trust. While the results were significant, $F(1,245)=7.05$, $p<.01$, the adjusted $\mathrm{R}^{2}$ indicates that less than $3 \%$ of the variance in social trust was explained by this spirituality-related variable.

Table 37: Stepwise regression of spirituality related variables on social trust

\begin{tabular}{llllll}
\hline Variable & $\mathrm{B}$ & $\mathrm{SEB}$ & Beta & $\mathrm{R}^{2}$ & Adjusted R $^{2}$ \\
\hline $\begin{array}{l}\text { Daily spiritual } \\
\text { experience with God }\end{array}$ & .048 & .018 & $.167^{* *}$ & .028 & .024 \\
\hline
\end{tabular}

Note: ${ }^{*} \mathrm{p}<.05,{ }^{* *} \mathrm{p}<.01,{ }^{* * *} \mathrm{p}<.001$

\subsubsection{Prediction of social trust}

In the second regression analysis, all the variables correlated with social trust shown in the correlation table were entered into the equation to see which variable(s) were significant predictors of social trust. Before entering into the regression equation, the assumption of regression analyses was examined. As previously, years attending church, time spent in church activities, and physical health, were transformed accordingly. Four univariate outliers and one multivariate outlier were identified and all five outliers 
were deleted from the analysis. The 10 variables were entered into the regression analysis, using the stepwise method.

Table 38 indicates that mental health, social cynicism, and sense of community were identified as significant predictors of social trust. Mental health was entered first into the equation, followed by social cynicism and sense of community. The results was significant, $\mathrm{F}(3,222)=14.38, \mathrm{p}<.001$, and the adjusted $\mathrm{R}^{2}$ indicates that about $15 \%$ of the variance in social trust is explained by the stated variables. As with the life satisfaction variable, no spirituality-related variables could be entered into the equation, indicating that no spirituality-related variables can contribute to explaining the variance of social trust on top of the three stated variables.

Table 38: Stepwise regression of variables predicting social trust

\begin{tabular}{llllll}
\hline Variable & $\mathrm{B}$ & SEB & Beta & $\mathrm{R}^{2}$ & Adjusted $\mathrm{R}^{2}$ \\
\hline Mental Health & .103 & .029 & $.226^{* * *}$ & .073 & .069 \\
Social Cynicism & -.348 & .085 & $-.251^{* * *}$ & .140 & .132 \\
Sense of Community & .129 & .052 & $.155^{*}$ & .163 & .151 \\
\hline
\end{tabular}

Note: ${ }^{*} \mathrm{p}<.05,{ }^{*} \mathrm{p}<.01, * * * \mathrm{p}<.001$

\subsubsection{Summary}

As with life satisfaction, although the correlation table and the first regression equation indicate that spirituality-related variables were significantly associated with social trust, and that the experiential side of spirituality can even predict social trust, the effect of the spirituality variable was cancelled out when the other key variables were taken into consideration. Thus, regression analyses seem to suggest that the effects of spirituality-related variables are again mediated by the relationships between mental health and sense of community to social trust.

\subsubsection{Relationship between Spirituality and Civic participation}

Pearson's product correlation (Table 32) indicates that civic participation is correlated with the following seven variables: membership of voluntary organizations, reward for application, religiosity (social belief), daily spiritual experience (DSES), sense of community (SCI), years attending church, and time spent in church activities. As fundamentalism correlates near zero with civic participation, but correlates significantly 
with other predictor variables (especially spirituality-related variables), it was identified as a suppressor variable (Schumacker \& Lomax, 2004). As Tabachnick and Fidell (2007) note, including a suppressor variable can enhance the effects of other independent variables in predicting the DV. This is because suppressor variables hide or suppress the "true” relationship between independent and dependent variables; by including this variable, some of the unwanted shared variance can be accounted for "by virtue of its correlations with other IVs” (p. 154). Therefore, as they suggested, fundamentalism was also included in the regression analysis.

\subsubsection{Prediction of spirituality related variables on civic participation}

In the first regression analysis, the association between spirituality related variables and civic participation was examined. Years of attending church and time spent in church activities were transformed because of the violation of normality assumption. While no univariate outlier was found, two other cases were identified through Mahalanobis distance as multivariate outliers with $\mathrm{p}<.001$ and were then deleted from subsequent analyses.

As the fundamentalism scale is a suppressor variable, it was entered into the equation before the other three spirituality-related variables (DSES, years attending church, and time spent in church activities) were entered as predictor variables, using the stepwise method (Tabachnick \& Fidell, 2007). The results (see Table 39) indicate that three spirituality related variables -- fundamentalism, daily spiritual experience and years attending church -- were all identified as significant predictors of civic participation. Because of the suppressor effect, fundamentalism yielded a negative beta weight, but it may account for some of the unexplained variance shared with daily spiritual experience and years attending church, which were entered next into the equation. While the results were significant, $\mathrm{F}\left(3,239=6.59, \mathrm{p}<.001\right.$, the adjusted $\mathrm{R}^{2}$ indicates that about $6 \%$ of the variance in civic participation can be explained by these spirituality-related variables.

Table 39: Stepwise regression of spirituality related variables on civic participation

\begin{tabular}{llllll}
\hline Variable & $\mathrm{B}$ & $\mathrm{SEB}$ & $\mathrm{Beta}$ & $\mathrm{R}^{2}$ & Adjusted R \\
\hline Fundamentalism & -.037 & .014 & $-.212^{* *}$ & .001 & -.004 \\
Daily spiritual experience & .038 & .015 & $.213^{* *}$ & .054 & .046 \\
Years of attending church & .296 & .122 & $.174^{*}$ & .076 & .065
\end{tabular}

Note: ${ }^{*} \mathrm{p}<.05 ; * * \mathrm{p}<.01, * * * \mathrm{p}<.001$ 


\subsubsection{Prediction of spirituality related variables on voluntary organization joined}

In the second regression analysis, the association between spirituality related variables and the number of voluntary organizations joined was examined. Years attending church and time spent in church activities were transformed as before, and inverse transformation was also applied to the number of affiliated organizations due to the violation of normality assumption. No univariate outlier was found, but two cases were identified through Mahalanobis distance as multivariate outliers with $\mathrm{p}<.001$ and were deleted from the subsequent analysis.

The four spirituality-related variables (fundamentalism, daily spiritual experience, years attending church and time spent in church activities) were entered into the regression analysis as predictor variables, using the stepwise method because of its exploratory nature (Tabachnick \& Fidell, 2007). The results (in Table 40) indicate that two spirituality related variables - fundamentalism, and years attending church -- were identified as significant predictors of membership of voluntary organizations. Fundamentalism was entered into the equation first and years of attending church followed. While the results were significant, $F\left(2,249=7.50, \mathrm{p}<.005\right.$, the adjusted $\mathrm{R}^{2}$ indicated that only about $5 \%$ of the variance in the number of voluntary organizations joined can be explained by the two spirituality-related variables. It should be noted that since the variable "number of voluntary organization joined” has been inversely transformed, the direction should be reversed in interpretation. In our case, while fundamentalism was shown to be inversely associated with membership of voluntary organizations, years attending church was shown to be positively associated. Table 40: Stepwise regression of spirituality related variables on voluntary organization membership

\begin{tabular}{llllll}
\hline Variable & $\mathrm{B}$ & $\mathrm{SEB}$ & Beta & $\mathrm{R}^{2}$ & Adjusted R $^{2}$ \\
\hline Fundamentalism & .004 & .001 & $.215^{* *}$ & .020 & .017 \\
$\begin{array}{l}\text { Years of attending } \\
\text { church }\end{array}$ & -.036 & .012 & $-.204^{* *}$ & .057 & .049 \\
\hline
\end{tabular}

Note: ${ }^{*} \mathrm{p}<.05 ; * * \mathrm{p}<.01, * * * \mathrm{p}<.001$

\subsubsection{Prediction of civic participation}


In the third regression analysis, all the variables correlated with civic participation were entered into the equation to see which variable(s) were predictors of civic participation. Years attending church, time spent in church activities, and numbers of voluntary organizations joined were also transformed. Two univariate outliers and one multivariate outlier were identified and all three outliers were deleted from further analysis. As fundamentalism is a suppressor variable, it was entered first into the equation before the other seven variables were entered using the stepwise method.

Table 41 shows that the number of voluntary organizations joined, daily spiritual experience, and time spent in church activities were significant predictors of civic participation. Voluntary organization membership was the first predictor entered into the equation, followed by daily spiritual experience and time spent in church activities. The result was significant, $\mathrm{F}(4,222)=21.41, \mathrm{p}<.001$. The adjusted $\mathrm{R}^{2}$ indicated that over one quarter (26.5\%) of the variance in civic participation can be explained. Fundamentalism was not shown to be a significant predictor by itself; however, because of its enhancing effect in predicting the dependent variable, it remained in the whole model. It is worth mentioning that while other spirituality-related variables influence civic participation positively, the influence of fundamentalism was negative.

Table 41: Stepwise Regression of variables predicting Civic Participation

\begin{tabular}{llllll}
\hline Variable & $\mathrm{B}$ & $\mathrm{SEB}$ & $\mathrm{Beta}$ & $\mathrm{R}^{2}$ & Adjusted R $^{2}$ \\
\hline Fundamentalism & -.023 & .013 & -.133 & -.001 & -.004 \\
Voluntary organization & -4.281 & .563 & $-.445^{* * *}$ & .231 & .225 \\
membership & & & & & \\
Daily spiritual experience & .030 & .013 & $.165^{*}$ & .265 & .256 \\
Time spent in church & .306 & .153 & $.149^{*}$ & .278 & .265
\end{tabular}

Note: ${ }^{*} \mathrm{p}<.05 ; * * \mathrm{p}<.01, * * * \mathrm{p}<.001$

\subsubsection{Summary}

The relationship between spirituality and civic participation is complex. Civic participation is shown to be significantly and directly predicted by the number of voluntary organization joined and three spirituality-related variables (fundamentalism, daily spiritual experience and time spent in church activities). However, fulfilling the conditions suggested by Baron and Kenny (1986), the effects of spirituality are also partially mediated through voluntary organization membership. Moreover, the effect of 
spirituality on civic participation can be both positive and negative. Fundamentalism is negatively associated with both the DV (civic participation) and the mediating variable (number of voluntary organizations joined), but other spirituality variables were positively correlated. Despite being a suppressor variable, fundamentalism enhances the overall adjusted $\mathrm{R}^{2}$ by its correlations with other IVs, as a result leading to its combination with other dimensions of spirituality becoming significant predictors of civic participation. Therefore, regression analyses suggest that the number of voluntary organizations joined, more daily spiritual experience, and time spent in church activities, but lower levels of fundamentalist belief, facilitate and predict civic participation. Furthermore, more years attending church, but lower levels of fundamentalism, increase the number of people joining voluntary organizations, and enhance civic participation.

\subsubsection{Path Analysis using AMOS}

The above results suggest that spirituality-related variables affect life satisfaction, social trust and civic participation, either directly or indirectly, through affecting sense of community, mental health and membership of voluntary organizations. To further examine the interrelation (direct, indirect and total effect) between variables, a path model (Figure 8) based on the theoretical framework (Figure 1), and findings drawn from multiple regression analysis, is proposed for testing using AMOS 7.0.

Compared to multiple regression, path analysis examines a more complex model including several dependent variables and mediating variables. Path analysis considers the interrelationship between all variables. Moreover, path analysis is not restricted to additive models, but can examine direct, indirect, and total effects, and is regarded as full, rather than partial, information analysis (Kline, 2005). Path analysis also considers error variance of dependent variables. Although path analysis has been viewed as a "reducedform” of analysis (Kline, 2005; Szaflarski, Leonard, Mrus, Peterman, \& Tsevat, 2005), compared to the full structural equation modelling, because of its vulnerability to the measurement error of independent variables (Kline, 2005; Schumacker \& Lomax, 2004), path analysis is widely used in psychological research, particularly in some conditions where full SEM cannot apply (Kline, 2005; Szaflarski, et al., 2005). 


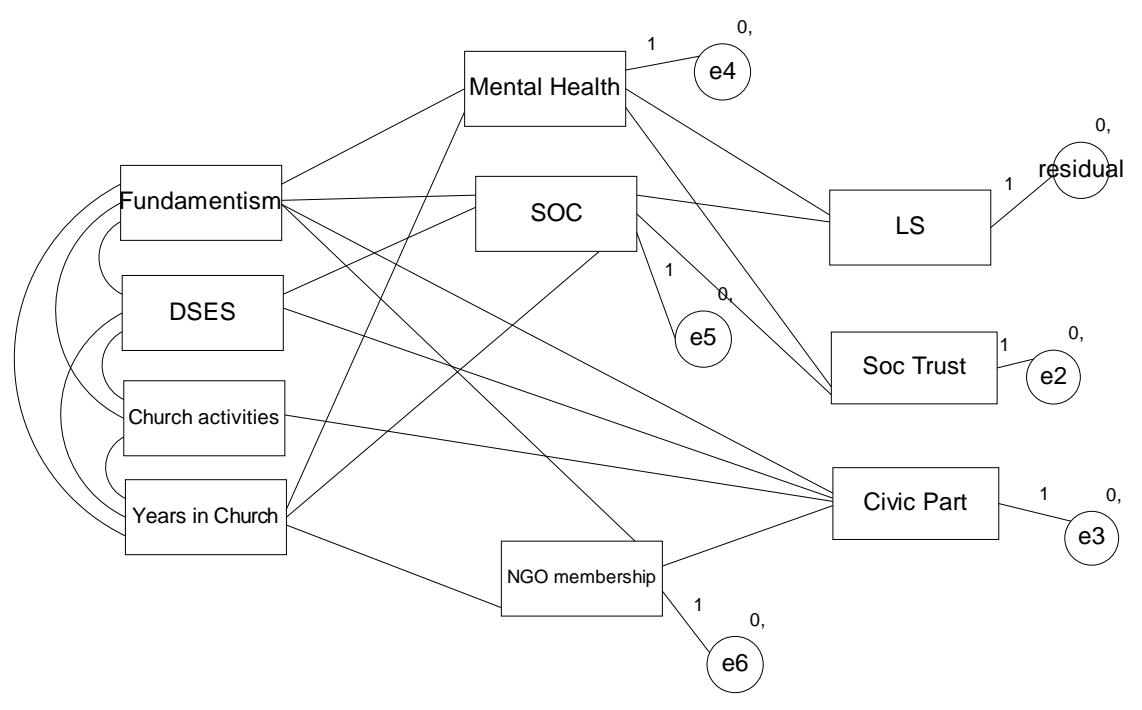

Figure 8 The hypothesized model of religion and well-being

\subsubsection{Preliminary Analysis}

As missing values can lead to huge data loss (98 cases and more than 5\%) and because the use of modification indices in AMOS program would require a full data set, expectation maximization (EM) provided by SPSS Missing Value Analysis (MVA) was employed to replace missing data. The SPSS MVA analysis indicates that two cases had missing value in more than half of the variables of the stated model (that is, more than 5 variables) and another 96 cases had missing values from 1 to 5 variables. As suggested by Holmes-Smith, Cunningham, and Coote (2006), the two cases with missing values for more than half of the variables were deleted. The other cases with missing data were replaced by expectation maximization (EM) imputation method. The non-significant result ( $\mathrm{p}=.293$ ) of Little's MCAR test provided by SPSS MVA output indicates that the missing pattern of the remaining cases are MCAR (missing completely at random) or at least MAR (missing at random). Therefore, analysis can proceed with confidence (Holmes-Smith, et al., 2006; Tabachnick \& Fidell, 2007). Furthermore, voluntary organization membership, years attending church, and time spending in church activities were transformed because of violation of normality, as mentioned before. In addition, two univariate and no multivariate outliers were also identified, resulting in a total of 320 cases for further analysis. Table 42 shows the means, standard deviations, and intercorrelations of the key variables of the path model. 


\subsubsection{Path Analysis}

The proposed path model (Figure 8) was evaluated via observed variable path analysis using maximum likelihood parameter estimation (AMOS 7.0). Since the goodness of fit index $\left(\chi^{2}\right)$ is sensitive to the sample size, three other fit indexes were also used to determine the adequacy of fit of the model, suggested by Meyers, Gamst and Guarino (2006). They are normed fit index (NFI), the comparative fit index (CFI), and the root mean square error of approximation (RMSEA). Specifically, models with NFI and CFI value at or above .95 and RMSEA values at or below .05 show an excellent fit to the data, while models with NFI and CFI values between .90 and .94 and RMSEA values between .06 and .10 show an adequate fit of the data. Values outsides these ranges indicate a poor fit of the model to the data.

The proposed model (A) (Figure 9) provided an adequate fit of the model to the data. Although the $\chi^{2}$ statistics was significant (42.23, $\mathrm{p}=.012$ ), relating to the significant difference between the predicted and observed relationship in the covariance matrix, the other three indices demonstrate an adequate fit of the model to the empirical data of the present study $(\mathrm{NFI}=.948 ; \mathrm{CFI}=.976$, $\mathrm{RMSEA}=.049)$.

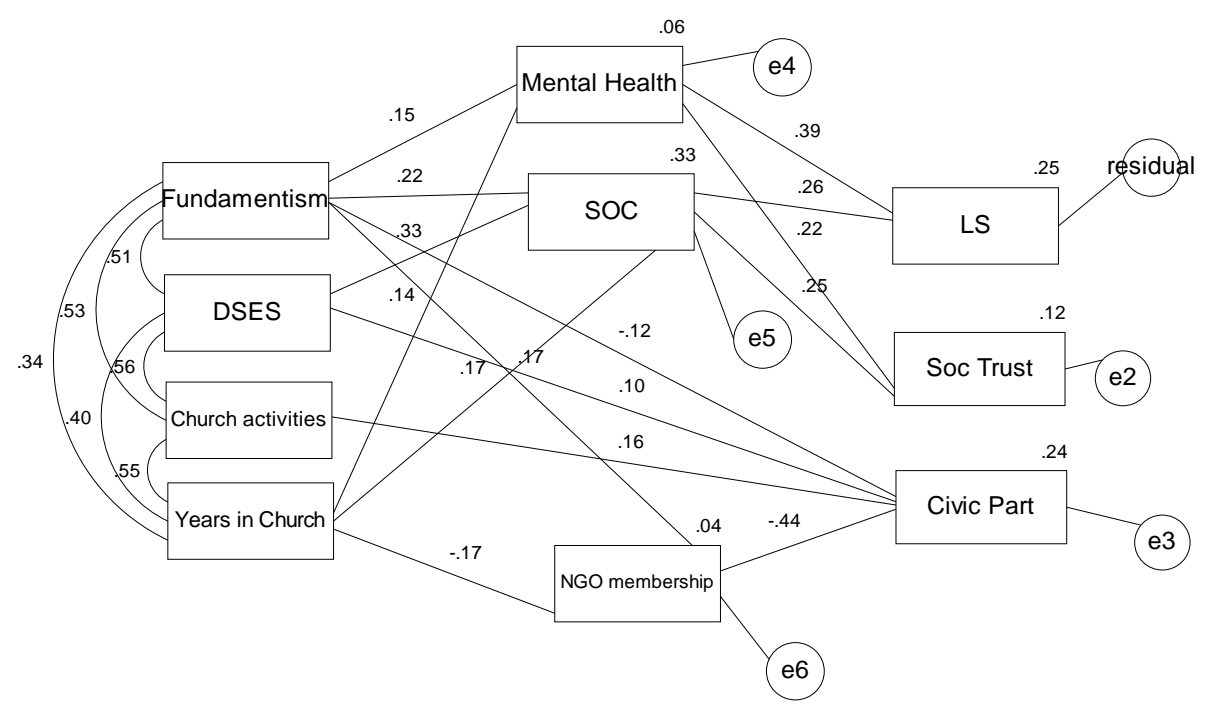

Figure 9 Path Model A 
Table 42 Means, Standard Deviations, and correlations among the variables in path model

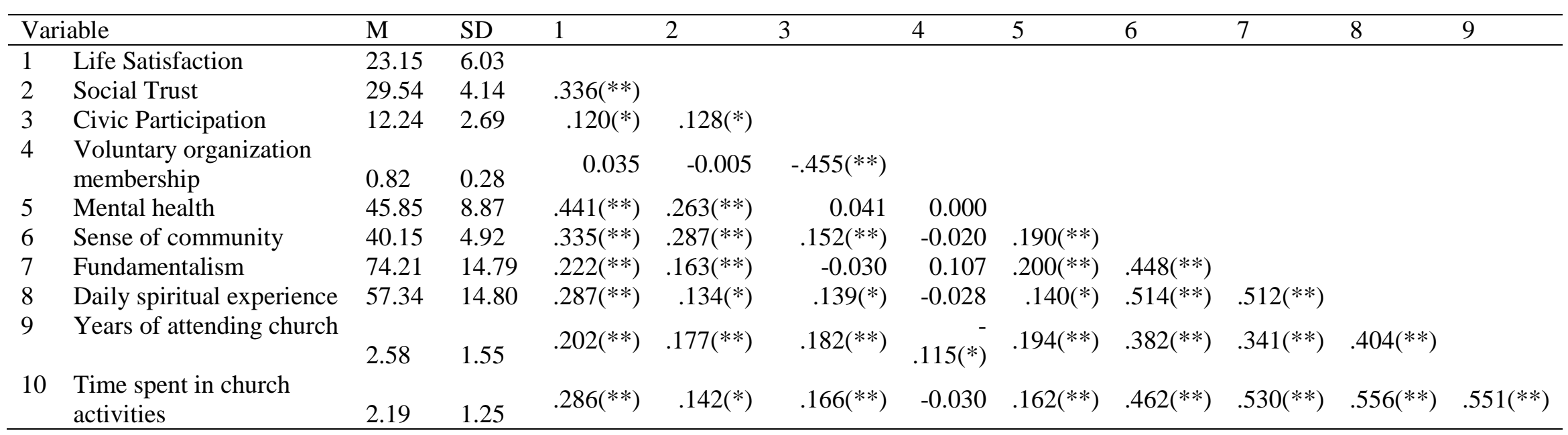

$* \mathrm{p}<.05 ; * * \mathrm{p}<.01, * * * \mathrm{p}<.001$ 
It is worth highlighting here, in Model A, that all the parameters of the path model were statistically significant, except the path from daily spiritual experience to civic participation. It should also be noted that spirituality-related variables are strongly associated with sense of community. Furthermore, while fundamentalism has significant positive association with mental health and sense of community, it has negative association with civic participation and voluntary organization membership. Moreover, the six exogeneous variables explained by predictor variables are: 25\% (life satisfaction), 12\% (social trust), and 24\% (civic participation), 6\% (mental health), 33\% (sense of community), 4\% (voluntary organization membership).

Nevertheless, as Schumacker (2006) understands it, although modelling testing can be conducted in a strictly confirmatory way, an initial model can also be modified (re-specified) on theoretical grounds, until a better fit is obtained by using specification search procedure. As suggested by the modification indices, two more path models with better fit, justified on theoretical grounds, were obtained (see Figure 10 \& 11). Table 43 lists the fit indices for all three models, along with chi-square difference test statistics for comparison of the fit of the nested model.

Table 43 Fit indices across endogenous variables, and chi-Square difference tests for the models

\begin{tabular}{llllllll}
\hline Model & $\chi^{2}(\mathrm{df})$ & $\mathrm{P}$ & NFI & CFI & RMSEA & $\chi^{2}$ difference $(\mathrm{df}=1)$ & $\mathrm{P}$ \\
\hline A & $42.23(24)$ & .012 & .948 & .976 & .049 & - & - \\
B & $29.61(23)$ & .161 & .963 & .991 & .030 & 12.62 & $<.005$ \\
C & $25.55(22)$ & .272 & .968 & .995 & .022 & 4.06 & $<.05$ \\
\hline
\end{tabular}

A second path model (B) (Figure 10), with the addition of a path from social trust to life satisfaction, was evaluated. The chi-square index is not significant (29.61, $\mathrm{p}=.161$ ), in combination with the other fit indices (NFI=.963; CFI=.991, RMSEA=.030), indicating a good model fit. The chi-square difference test comparing model A and B indicated that Model B provided a significant improvement in the model fit, $\chi^{2}(1)=12.62$, $\mathrm{p}<.005$. 


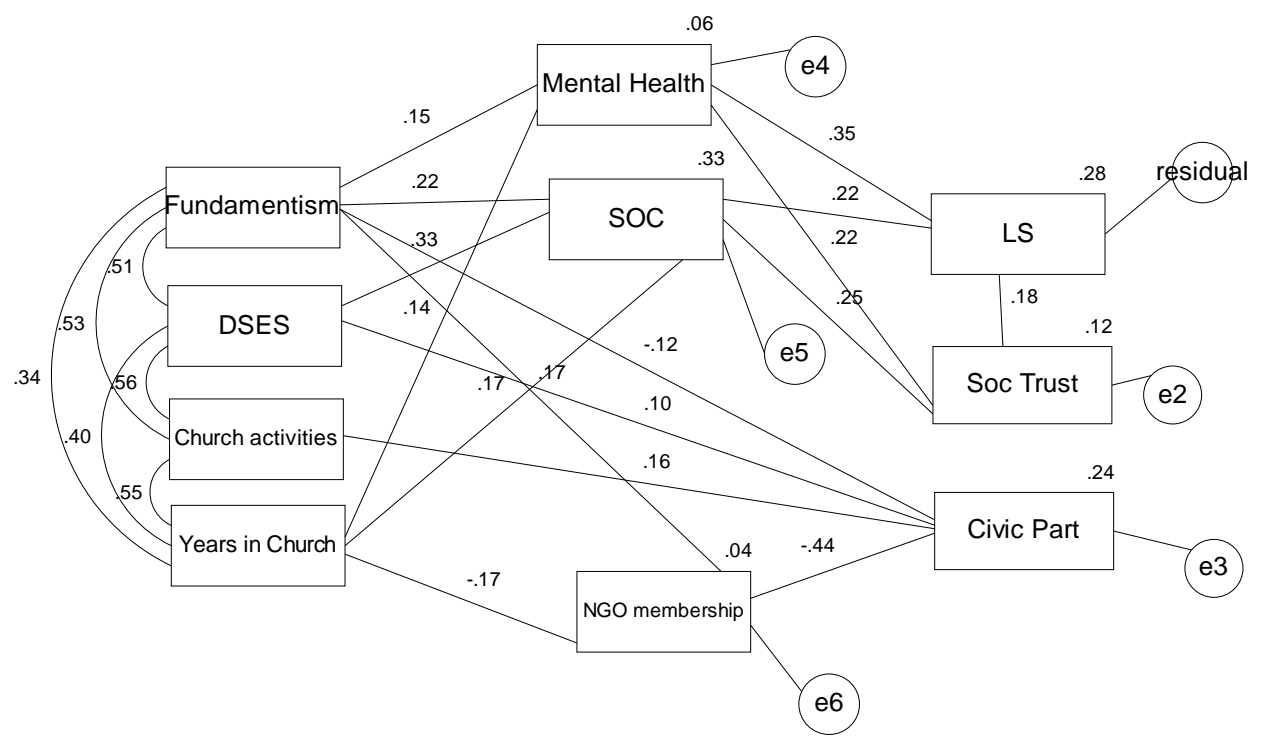

Figure 10 Path Model B

Model C (Figure 11) was identical to Model B, with the addition of a path from the time spent in church activities to sense of community. Model C provided an excellent model fit to the sample data, $\chi^{2}(22)=25.55, \mathrm{p}=.272$, NFI=.968; CFI=.995, RMSEA=.022. Chi-square difference test comparing $\mathrm{B}$ and $\mathrm{C}$ provided a significant improvement in model fit $\left(\chi^{2}(1)=4.06, \mathrm{p}<.05\right)$. The modification indices suggested no more significant paths were likely to emerge from further modelling testing. Compared to Model A, the variances of six exogenous variables in Model C explained by other predicting variables are primarily the same, except in life satisfaction (from 25\% to 28\%), and sense of community (from 33\% to 34\%).

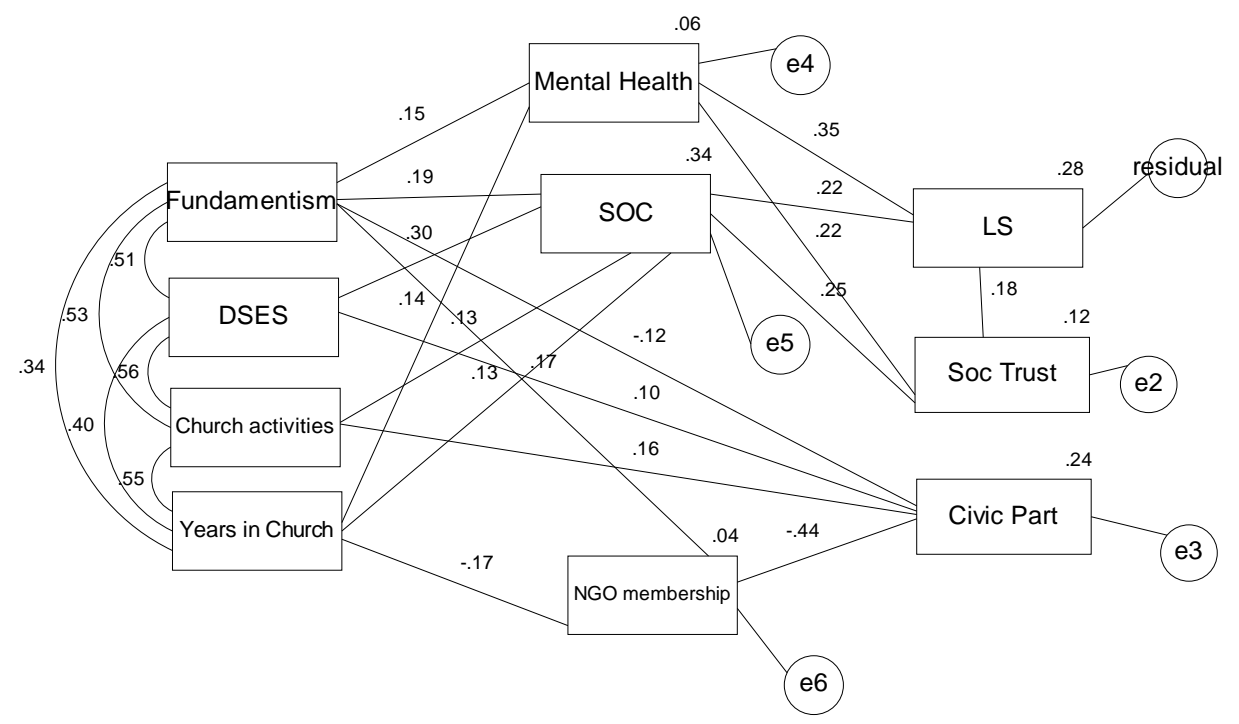

Figure 11 Path Model C 
In addition, when examining the parameters of the path model, like Model A, all hypothesized paths are statistically significant, except the path from daily spiritual experience to civic participation. Nevertheless, it is worth explaining here the negative path from fundamentalism to civic participation. Since the path coefficient has a greater absolute value than its bivariate correlation with the civic participation, and from regression analyses (section 9.5.3), we know that the negative correlation of fundamentalism in the preset data indicates the presence of statistical suppression (Kline, 2005). In this case, fundamentalism functions as a suppressor in relation to other spirituality variables. That means, after the overlapping variance among fundamentalism, daily spiritual experience, time spent in church activities and civic participation are accounted for, the remaining variance in fundamentalism was negatively related to civic participation. Therefore, we discover that spirituality has both a direct negative effect (fundamentalism belief) and positive effect (daily spiritual experience and time spent in church activities) on civic participation and, at the same time, an indirect positive and negative effect (that is, mediated through the number of voluntary organization joined).

Ideally, separate path models would have been run for each setting to examine how well the model applied to different type of spirituality context. However, path analysis is a kind of large sample technique, with estimates of the parameters unstable if the sample size is too small (Kline, 2005; Streiner, 2005; Tabachnick \& Fidell, 2007). A general guideline regarding sample size is 10 times the number of free parameters or at least more than 100 cases (Kline, 2005). Such analyses could not be reliably run in the present study because of the sizes of sub-samples. Models for each of the five settings are included in Appendix 2 as reference, but any interpretation should be cautious.

\subsubsection{Summary}

The results of this study support the theoretical framework that spirituality-related variables influence well-being indirectly or directly, but that depends on the kind of wellbeing outcome. In terms of life satisfaction and social trust, spirituality-related variables are mainly mediated by sense of community, and mental health, which directly predict well-being. No single spirituality variable affects well-being directly. In terms of civic participation, the influence of spirituality is both direct and indirect. Although spirituality can influence civic participation directly, religious influence can also be mediated 
through voluntary organization membership. Moreover, it is worth noting here that religious influence can be both positive and negative. While, fundamentalist belief was inversely associated with civic participation and voluntary organization membership, other spirituality related variables were shown to be positively associated. 


\section{Chapter 10}

\section{Discussion of the Quantitative Study}

This study examines the impact of spirituality and religious community on wellbeing. The section is divided into three parts. The first, drawing on the multivariate ANOVA findings, discusses the effect of setting on a range of outcome variables. The second, based on discriminant analysis findings, explores the underlying dimensions differentiating various settings. The third, based on the modelling testing results, discusses the complex, rather than linear, relationship between religion and well-being.

\subsection{Comparisons between settings}

\subsubsection{Well-being}

The multivariate ANOVA findings in this study indicate that members in a spiritual context have slight, but significant, higher level of well-being, manifested in life satisfaction, social trust, membership of voluntary organization memberships, civic participation and mental health, but not in physical health.

Regarding life satisfaction, social trust and mental health, religious members display higher scores than those belonging to the social centre, although not all church members are significantly different from their social centre counterparts. Regarding life satisfaction, members of three churches - the charismatic church (CHC), social concern church (SCC) and the evangelical church (EVC) - demonstrate higher and significantly different results from their social centre counterparts, while the fundamentalist church (FDC) have no significantly different results from all other groups. Regarding mental health, only social concern church members exhibit significantly higher scores than social centre members, while the other churches have no significant difference with other settings. These findings are consistent with previous studies showing the positive influence of religion on life satisfaction, positive emotion and mental heath (Diener, 1984; Diener, et al., 1999; Hackney \& Sanders, 2003; Koenig, 2005; Myers, 2008). The positive and persistent influence of religion on health and well-being (individual level) may explain why, in the past decade, the relationship between religion, health and well- 
being is gaining more attention. Many special issues of psychological journals have also focused on the topic: Psychological Inquiry (Baumeister, 2002), Mental Health, Religion and Culture (Joseph, Linley, \& Maltby, 2006), American Psychologist (W. R. Miller \& Thoresen, 2003), Journal of Community Psychology (Kloos \& Moore, 2000a; Moore, Kloos, \& Rasmussen, 2001). It has also been observed that recent discussion on religion and health and well-being has shifted focus from whether to how religion can heal (Kluger, 2009). The differential influence of various religious settings, shown in the present study, may reflect different pathways or mechanisms in various religious settings.

The influence of settings on social trust is more complex. Fundamentalist church members demonstrate a higher and significant difference compared to counterparts in the social centre and evangelical church, but there were no significant differences between the social concern church and the charismatic church and all other settings. The higher score of social trust, despite not always being significant in all religious settings over non-religious settings, seems to support Newton's claim (2004) that “culture (or institute) strongly influenced by the Protestant ethic are more trustful than others”(p. 30). However, if the Protestant culture really matters, why does just one church (the fundamentalist church) stands out significantly? Moreover, why does the evangelical church fall behind all the other churches? The outstanding performance of the fundamentalist church and the middling performance of evangelical church members require further exploration.

Welch et al. (2004) posit that conservative religious groups do not hinder people building social trust with outsiders, and they also argue that strong social bonding within the strict community enables extends trust to people outside their community. Fundamentalist church members' higher social trust (outlined in the present study) seems to support this argument. Nevertheless, the evangelical church can also be regarded as a strict community in terms of members' religious fundamentalist beliefs, church attendance, and participation, and so we can ask why its members' levels of social trust are significantly lower than that of the fundamentalist church. Putnam (2000) suggests that the scale of communities may matter, and Calhoun-Brown (2005)'s empirical study confirms the variation between social trust and church size. Her research demonstrates that the relationship between church size and social trust appears to be curvilinear. She found that members belonging to churches with fewer than 200 and more than 1000 people were less trusting. She explains that very small and large churches have can 
inhibit people building trust, because "individuals who attend very small churches may not be willing to extend trust to others and people who go to very large ones may not develop the requisite relationship or have the necessary amount of personal interaction to facilitate trusting bonds” (p. 13). Her explanation accords with the finding in the present study because members in both the social concern church (a congregation with fewer than 100 people) and the evangelical church (more than 1000 people) have lower levels of social trust compared to the fundamentalist church. Therefore, that seems to suggest that, in addition to the type of religious communitity, structural variables like church size may also have influence on members’ social trust.

Regarding civic participation and membership of voluntary organizations, the social concern church (SCC) significantly stands out among all other groups (be they religious or non-religious). Members of the social centre (non-religious group) participate more in civic activity than other church group members, but the difference is not statistically significant. The finding is consistent with Wuthnow's observation (1999) in two ways. First, the standing out of SCC supports the fact that religion enhances civic participation. The ordinary, if not worse, levels of civic participation in other evangelical typed communities confirm Wuthnow's argument that the composition of religious participation matters and affects civic engagement. In the present study, the findings can be explained in two major ways. The first relates to the nature of the control group -social centre, in which nine participants joined the volunteer groups of the centre. The network and affiliation with other organizations provided by the center help social centre members to build more impressive civic participation compared to the general population. The other reason may be due to limited time available to people. As Li, Pickles and Savage (2005) note, social capital is necessarily exclusive because the networks generated within a group also involve non-ties with outsiders. Therefore, when people in the church participate more in the church, they have less time available for other settings. As Wuthnow (1999) argues, it is bonding rather than bridging social capital that is developed within evangelical and fundamentalist communities.

Regarding physical health, there are no significant differences between all settings, either religious or non-religious. Members of the three churches (charismatic, social concern and evangelical) demonstrate a higher value than those in the social centre. However, FDC members demonstrate a lower value in this dimension than counterparts 
in all other settings. The finding seems to contradict the generally positive association between religion and health (Koenig, et al., 2000).

In summary, the generally positive influence of religion on well-being is maintained. The effect of religious influence, however, is not as strong. Nevertheless, the present study clearly shows that the influence of religion spans various dimensions, including subjective well-being (life satisfaction), and psychological and social wellbeing (social trust and civic participation). Moreover, the significant influence of religion on well-being is not the same across different religious settings. Despite similar levels of spirituality (the charismatic, fundamentalist, and evangelical churches), the manifestation of well-being can be quite different across religious settings. Different religious settings may nurture different kinds of well-being.

\subsubsection{Spirituality}

Regarding spirituality, the findings of this study demonstrates that, as expected, spiritual context is significantly and abundantly associated with spiritual outlook, manifested in fundamentalist belief, daily experience with God, years attending church and time spent in church activities. Religious setting apparently has a medium to large influence on members' spirituality variables. Moreover, compared to members in the social centre, members in all kinds of religious settings exhibit higher and significant level in all spirituality variables, except the fundamentalism scale. However, there are still significant differences within church settings: the influence of spiritual setting on spirituality is differential.

Regarding the fundamentalism scale, fundamentalist, charismatic and evangelical church members demonstrate a significantly higher result than their counterparts in the social centre and social concern church while there are no significant differences within the two clusters. This accords with the claim of Altemeyers and Hunsberger (2004) that people with a high score related to religious fundamentalism are "particularly concentrated in "fundamentalist" Protestant denominations, such as Baptists, Mennonites, Evangelicals, Pentecostals, Jehovah’s Witnesses, the Alliance Church, and so on (p. 49)”. It is noted that Protestant Christians in Hong Kong are generally from a fundamentalist background (Ying, 2004). 
Regarding daily spiritual experience, significant differences are observed within church settings. While those in the charismatic and fundamentalist church exhibit a significant higher score than those in the social concern church, members in the evangelical church have no significant difference in their DSES score compared to those in the other three churches. The author understands that no previous empirical study has examined the experiential dimension of different types of congregation, let alone the church in Hong Kong. However Lazar’s (2004) study of religious experience among 165 Jews from Ashkenazi, Sephardic and Ethiopian cultural backgrounds indicates that cultural background influences religious experience and motivation. Poloma and her colleague (Poloma, 1995; Poloma \& Pendleton, 1991) suggest that denominational affiliation is related to religious experience, as conservative Protestants tend to have more experience than Catholics or mainline Protestants. The present study seems to suggest that, as well as ethnic-cultural or denominational background cultural ethos in the immediate setting also affects religious experience.

Regarding time spent in church activities, the present study also indicates similar findings. Those in the charismatic, fundamentalist and evangelical church exhibit a significantly higher score than those in the social concern church. As briefly mentioned before, Wuthnow (1999) observes that evangelical type religious communities help develop a strong bonding capital within the church at the expense of bridging capital that can have a larger impact on society. The finding in the present study accords with that argument, by indicating that members in these religious settings spent significantly more time in church activities, compared to their non-religious counterparts or those in churches with a social concern orientation.

Regarding years attending church, members in the social concern and fundamentalist church have a longer history of attending church than those in the charismatic and evangelical church. More than half of the members of the fundamentalist church had attended the church for 10 to 20 years; over $60 \%$ of evangelical church and charismatic church members had attended church from 1 to 10 years. Length of attendance at the social concern church is clustered around two ranges: from 1 to 5 years, and over 20 years. These patterns not only reflect specific church culture, but also unique church history that is explored in detail in the qualitative section. 
Besides the four studied spirituality variables, chi square findings indicate that there is a significant and strong association between settings and religious practice, manifested in prayer and meditation, bible reading, serving in the church, and church attendance. As expected, people in the religious settings have a significantly higher level of religious practice compared to those in non-religious setting. However, even within the religious setting, there are significant differences related to three kinds of practices (prayer and meditation, reading the bible, and church attendance). As with other spirituality related variables, social concern church members have a lower level of religious practice compared to those in other churches.

In summary, the findings of the present study accord with Kelley’s (1972) observation that conservative churches are strong. Compared to members in churches with a social concern orientation, members in the evangelical, charismatic and fundamentalist churches not only spend more time in church activities, pray and meditate more, read the bible and attend church more frequently, but also experience God on a daily basis more frequently. They also demonstrate a higher level of fundamentalist belief towards their religious faith. These findings apparently support Kelley’s reluctant claim that strength and strictness are highly correlated, if not inevitably concomitant. However, instead of viewing the strictness of religion negatively, it can be said that religious communities give members many opportunities to participate and feasible ways to communicate with the divine. All these strengthen their religious claims, even though those may be "strict" for outsiders. Whether strictness of spirituality is the by-product or the source of the strength of religion is not very clear, and needs further examination. Overall, the findings of this study further confirm the fact that spiritual context influences members' spirituality, whether in belief, experiential or behavioural dimensions, but the influence differs according to religious setting.

\subsubsection{Sense of community and social belief}

Multivariate ANOVA findings in this study also indicate that the spiritual context is moderately but significantly associated with members' sense of community and some social beliefs, expressed in fate control and religiosity. However, spiritual context does not significantly associate with other social beliefs, including social cynicism, social flexibility, and reward for application. 
Regarding sense of community, members in all kinds of religious setting have a stronger sense of community than counterparts in the social centre. However, charismatic and fundamentalist church members have a significantly stronger sense of community than their counterparts in the social center, and social concern and evangelical churches. These findings reflect two facts. Firstly, religious setting, without doubt, plays a significant and contributory role in nurturing a sense of community towards immediate setting. This accords with Durkheim's (1912/2001) idea that religion is the soul of society and community. As he argues, religion contains and generates everything that gives rise to collective life, such as myths and legends, ritual practice and ceremony, law and morality. Nevertheless, secondly, the present study also indicates other facts - how the extent to which a sense of community is nurtured in various religious settings can differ. This may be related to multiple factors. The literature on sense of community (McMillan \& Chavis, 1986) reminds us to closely examine membership, influence, integration, fulfilment of needs, and shared emotional connection, within the setting to gain deeper understanding. Moreover, drawing on social identity theory, Herriot (2007) explains how building a strong in-group identity can help the fundamentalist group maintain selfesteem and reduce uncertainty when facing challenges or threats. While we will look at more detail regarding the internal mechanisms of each church in qualitative study, but church size and church culture may be critical factors.

Regarding religiosity (social belief), as expected, members of all four churches demonstrate higher level of religiosity than those at the social centre. The findings accord with those in our previous section about the significant effect of spiritual context on spirituality. However, within religious setting, the charismatic and evangelical church members have a significantly higher degree of religiosity than social concern church members, while fundamentalist church members are not significantly different in that respect compared to other churches. The result is slightly different to previous findings related to fundamentalist belief in which the fundamentalist church was significantly different to the social concern church. While the Religious Fundamentalism Scale adhere to religious belief (Altemeyer \& Hunsberger, 2004), religiosity measures the social axiom scale, tapping items related to the existence of supernatural forces and the benefits of religious belief (Leung \& Bond, 2004, p. 12). The relatively lower religiosity of fundamentalist church members, in contrast to fundamentalism, seems to suggest that 
fundamentalist church members believe strongly in their religious faith but do not benefit fully from their faith, or they realize that religion is not necessarily beneficial to all kinds of people, as compared to their evangelical and charismatic church counterparts.

Regarding fate control, the fundamentalist and evangelical church members have a significantly lower rating than those in the social concern and the social centre.

Although charismatic church members have a significantly lower rating than those in the social centre, they have no significant difference with their counterparts in different churches. Religion has a generally positive influence in mitigating fatalism, particularly for evangelical churches. This accords with existing findings that suggest fate control is negatively correlated with traditional Christian beliefs (Singelis, et al., 2003) and that religion can address negative and pessimistic views often associated with fatalism, particularly related to cancer research (Morgan, Tyler, \& Fogel, 2008). Fate control measures the belief in life being predetermined by something that beyond individual control and is positively correlated with an external locus of control (Leung \& Bond, 2004, p. 12). The findings suggest that people in religious groups are more self determined, particularly the evangelical and conservative groups. This may be due to a belief that individual fate is in God's hands, rather than being external and unknown.

In summary, the findings of the present study support the notion that religious settings have a positive influence on nurturing a sense of community and certain types of social belief. Compared to their social centre counterparts, members belonging to religious groups not only have a higher sense of community, but also have a stronger sense of religiosity but lower feelings of being controlled by fate. Moreover, even though social axioms, understood as general beliefs that people hold as a social experience, are often used as a comparison in cross-cultural studies (Leung \& Bond, 2004), the withinculture data (as in the present study) further support the notion that that the immediate settings the individual is embedded in can strongly influence general social beliefs. Specifically, in this study, the religious setting was shown to strongly influence belief related to religion and fate control.

\subsubsection{Summary}

Overall, multivariate ANOVA findings indicate that religious settings have a large influence in nurturing spirituality and a moderate effect on enhancing sense of 
community or mitigating fatalism. However, the influence of religious setting on wellbeing is small, but still significant. Moreover, not all kinds of religious settings have the same influence on well-being. Members of the charismatic church have a significantly higher level of satisfaction with life; those in the fundamentalist group have significantly higher levels of social trust, and members of the social concern church have higher levels of civic participation. Therefore, we can say the influence of religious setting on wellbeing is not linear but differential across religious settings. That makes examining the underlying dimensions across various settings valuable and meaningful.

\subsection{Dimensions differentiating the settings}

\subsubsection{Taxonomy of settings}

Discriminant Analysis (DDA) provides us with four discriminant functions along which the five settings separate from each other. The first function is defined primarily by spirituality related variables (fundamentalist belief, time spent in church activities, daily spiritual experience, religiosity, and fate control). The linear discriminant function (LDF) plot indicates that the first function separates the social centre from other religious groups. The second function is primarily defined by a mixture of well-being and spirituality related variables (years attending church, number of affiliated organization joined, civic participation and mental health). This function separates the social concern church from the other religious groups. The third function is defined primarily by sense of community and social flexibility, mainly contributing to the separation of the evangelical church from the other two churches (the charismatic and fundamentalist church). The last function is defined by a mixture of well-being variables and social beliefs (reward for application, physical health, social trust, life satisfaction, and social cynicism). Despite its minor effect, it helps explain the differences between the charismatic and fundamentalist church.

Descriptive discriminant analysis gives us a heuristic understanding of the five studied settings. The first function — spirituality related variables — can help us easily separate the non-religious from the religious settings. It also suggests that the social concern church is in between the social centre and the other three churches in terms of the spirituality related dimension. The other derived functions also further our understanding of the unique characteristic of different studied churches. The second function tells us 
that (compared to other church members), social concern church members attend church longer, participate more in civic organization and activities, and have better mental health. Evangelical church members, in contrast, have a relatively weaker sense of community, and greater social flexibility than their charismatic and fundamentalist church counterparts. Charismatic church members have better physical health, greater life satisfaction and an attitude of reward for application, but lower levels of social trust and social cynicism, compared to fundamentalist church members.

Taxonomies between different religious settings have been carried out before. As mentioned in Chapter Four, the fundamentalist-liberal continuum is commonly used to classify three major types of denominations: fundamentalist, moderate and liberal denominations (T. W. Smith, 1990). Other typological approaches to congregations can also be found. For example, Roozen et al. (1984) categorises congregations into four types: sanctuary, evangelist, civic and activist. Dudley and Johnson (1993) inductively discover five self images that the congregations relate to their community: prophet, pillar, servant, survivor and pilgrim. Becker (1999) identifies four fundamental congregational identities: leader, community, family, and house of worship. Carroll and Roozen (Carroll \& Roozen, 1990) outline six types of organizational identities within the Presbyterian church: old first Presbyterian, civic, evangelical, activist, sojourner, and family.

The findings of the present study resonate with the typology in these literatures. The social concern church is at the liberal end, and the other three churches range from moderate to fundamentalist. SCC is what Roozen et al (1984) describe as activist, or alternatively it is Dudley and Johnson’s prophet church (1993), or Becker’s leader congregation (1999), focusing on civic participation; the charismatic and fundamentalist church can be categorised as Becker’s family congregation, or Carroll and Roozen’s family identity, because of a higher sense of community within the setting and nonactivist orientation. The present study also accords to a recent study by McCrohan and Bernt (2004), examining service and worship as models of religious expression among Catholic college students. Also using Descriptive Discriminant Analaysis, that study successfully distinguished the service-oriented, worship-oriented, and uncommitted groups along two dimensions: questioning and doctrinal. They found that the serviceoriented group emphasizes social justice and spiritual awareness, and also adopt a more open attitude towards religious faith, viewing religion as a quest. Instead, the worship- 
oriented group is more aligned with traditional Catholic belief concerning prayer, sacrament, scripture, or moral values. In the present study, the social concern church is like the service-oriented group, with greater participation in civic activities and with a relatively lower level of fundamentalist belief as well as other spirituality-related variables. The other three churches are like worship-oriented groups, characterised by greater fundamentalism, daily spiritual experience, and participation in church activities.

The present study adds another dimension to differentiate the churches $-\mathrm{a}$ dimension related to the connectivity of the group. The study indicates that compared to evangelical church members, charismatic and fundamentalist church members have a greater sense of community within the settings. They, however, are also less flexible in viewing the multiple realities of the world, or different ways of achieving a given outcome. This may reflect the closely-tied environment. In contrast, EVC, because of its size and lower level of connectivity, cannot maintain a strong sense of community, but allows more diversity and flexibility. This seems to suggest that size, and ties or connectivity, are important areas to explore.

The present study also suggests that within family-type churches (charismatic and fundamentalist churches) we can identify two types of congregation. One is characterized by life satisfaction, reward for application, better physical health. and less social cynicism; the other scores higher in social trust. The charismatic church members are relatively more satisfied with life, believe more in effort, are less sceptical towards life, and have better physical health, while fundamentalist church members show a relatively higher level of social trust. Nevertheless, as discriminant analysis indicates, the fourth function only explains $4.2 \%$ of variance. Whether the function has further implications beyond the studied sample is not certain.

\subsubsection{Summary}

To conclude, discriminant analysis demonstrates several key aspects relevant to the present study. Firstly, spirituality related variables are useful to distinguish nonreligious groups from religious groups. Secondly, the social orientation of the setting is helpful to identify the activist and socially-concern church (SCC) from other evangelical religious settings. Other more refined categories related to theology or mission (such as civic or sanctuary orientation) have not been identified because of the selected area of 
inquiry of the present study. Thirdly, sense of community (combined with weaker social flexibility) emerges as another key dimension - relating to church size and connectivity of the setting - which is worth further scholarly exploration. Finally, within the family type religious setting, various expressions of spirituality and well-being indicate the richly diverse, if not intricate, outlook of religious congregations.

\subsection{The association between spirituality on well-being}

Multiple regression and path analyses indicates that spirituality-related variables are associated with well-being, either directly or indirectly or in a combined way, through the mediation of sense of community, mental health, and number of voluntary organization joined. Although several outcome variables may not be totally or easily separated from each other, we will delineate below each well-being variable separately before we finally examine the whole path model.

\subsubsection{Prediction of Life satisfaction}

In regression analysis it was discovered that physical health, mental health and sense of community are key predictors of life satisfaction. While the direct effect of spirituality on life satisfaction is absent, the mechanism of their influence is more complex. Although spirituality related variables are significantly associated with life satisfaction, their predictive power was cancelled out once the three stated key variables were considered. Nevertheless, the three spirituality variables (fundamentalism, daily spiritual experience and years attending church) are still highly associated with, and predictive of, sense of community, while the participants' fundamentalist belief and years attending church are also significantly associated with mental health. These findings suggest that the relationship between spirituality and life satisfaction is mainly mediated through influencing sense of community and mental health.

The salient relationship between sense of community and life satisfaction is supported by Prezza and colleagues (Prezza, Amici, Roberti, \& Tedeschi, 2001; Prezza \& Costantini, 1998), particularly regarding smaller communities. The relationship between health and life satisfaction also has been heavily documented (Diener, 1984; Diener, et al., 1999). The findings of the present study strengthen these lines of argument. 
Regarding the relationship between religion and mental health, Koenig and his colleague (Koenig, et al., 2000; Levin, 2001) have extensively documented a positive relationship between religion and mental health. Hackney and Sanders (2003), using meta-analysis, argue that "regardless of any considerations of religiosity or mental health definitions, religiosity may be said to have a salutary relationship with psychological adjustment.” (p. 51). Regarding religion and sense of community, although the linkage between religion and the emergence of community has long been acknowledged (Durkheim, 1912/2001), empirical findings are rare (see Obst \& Tham, 2009 for exception). Therefore, the findings of the present study further supports our the literature linking spirituality with enhanced mental health and sense of community.

To the author's knowledge, the present study is the one of the few, if not the first, study examining the relationship between spirituality well-being through an understanding of the mediated effect of the sense of community and mental health. Regarding the connection between religion and mental health, most recent efforts focus on understanding the mediating role of meaning in life (Blaine \& Crocker, 1995; N. K. Vilchinsky, S. , 2005; Whitford \& Olver, 2009), coping strategy (Frabricatore, Paul, Doris, \& Frank, 2004), perceived health (Zullig, Ward, \& Horn, 2006), goal (Emmons, 1999, 2005; Fiorito \& Ryan, 2007), optimism and social support (Salsman, Brown, Brechting, \& Carlson, 2005). The present study makes an important contribution to understanding this connection, although the linkage between religion and sense of community and mental health has long been recognized. Nevertheless, given the positive influence of mental health on life satisfaction shown in the present study, the direction of influence between the two variables is still debatable.

What is worth highlighting here is the significant contribution of two spirituality dimensions on mental health: fundamentalist belief and the years attending church. It is understandable that length of time attending church is associated with mental health because the variable may reflect general religiosity incorporating the time dimension. It is aligned with the work of Obst and Tham (2009) within a church community: they argue that religiosity is significantly related to length of membership. What is not expected, in fact quite surprising, is the predictive power of fundamentalist belief on mental health compared to other religious dimensions (such as the relationship with God dimension). Hackney and Sanders's (2003) meta-analysis indicated that in the pattern of relationships 
between religion and mental health, personal devotion has a much stronger effect than ideology or institutional religiosity. Given the effect of religious belief, fundamentalist belief is often associated with negative outcomes, such as prejudice (Altemeyer \& Hunsberger, 1992), particularly towards homosexuals (Bruce. Hunsberger \& Jackson, 2005; Laythe, Finkel, \& Kirkpatrick, 2001), right-wing authoritarianism (Altemeyer \& Hunsberger, 1992; Shaffer \& Hastings, 2007), dogmatism (Saroglou, 2002c), closedmindedness, or even a lack of humour (Saroglou, 2002a, 2002b; Saroglou \& Jaspard, 2001). The findings of the present study, however, indicate that fundamentalism belief, rather than other more "humane" religious dimensions (such as daily spiritual experience), is associated with mental health. This can be understood in two ways.

The first one is the mitigating effect of fundamentalist belief on anxiety or doubt over existential issues. As Hunsberger, Alisat, Pancer and Pratt (Bruce. Hunsberger, Alisat, Pancer, \& Pratt, 1996) indicate, fundamentalists were shown to have less doubt about God or religion per se than the less fundamentalist believers. Friedman and Rholes (2008) also show that religious fundamentalism can "serve a protective function against existential anxiety.”(p. 36). These findings accord with Batson et al (1993) who argue that those who adopt a "quest" religious orientation tend to experience more distress and anxiety. Following this, the second line of argument is about the stabilizing, if not enhancing, effect provided by fundamentalist belief on psychological health. Saroglou (2002c) observes that, although religious fundamentalism is positively associated with dogmatism (measured by a need for closure scale), it was also found that the strongest relationship between religion and dogmatism is centred around two facets: preference for order, and preference for predictability. Saroglou explains that what religious fundamentalist people dislike is "disorder among ideas, the chaos in the inner world, the simultaneous presence of incompatible elements, and the lack of integrationsubordination of everything to what constitutes the fundamental, essential body of their belief system"(p. 185). Instead, what they are looking for is "meaning that offers consistency, order and integration” (p. 185), which can be satisfied by fundamentalist belief. The salutary and stabilizing effect of religious fundamentalist belief was explored in a recent study based in South Africa (Pienaar, Beukes, \& Esterhuyse, 2006) in which religious fundamentalism (in combination with anti-hedonism) was shown to be predictive of psychological well-being (in terms of sense of coherence, satisfaction with 
life, and level of fortitude) in adolescents. Pienaar et al. explain that conservative and fundamentalist belief gives adolescents a stable internal structure to develop well-being, especially in times of great social change. Hood, Hill and Williamson (2005) have a similar argument. They argue that fundamentalism is a meaning system, derived from historical tradition and drawing heavily on sacred text, which allows people to persevere in a challenging environment and gives them a way and framework to make sense of themselves and their relationship with the world. Besides the stabilizing effect, Sethi and Seligman (1993) further demonstrate the enhancing effect of fundamentalism in their study, in which fundamentalists were shown to be more optimistic than people who had moderate or liberal religious belief. The present study suggests that, instead of having a negative effect, fundamentalist belief can benefit mental health. The SF-12 mental health dimension, used in the present study, primarily measures the people's role emotion, perception of mental health and social functioning, and it is believed that fundamentalist belief also has a salutary effect, not only stabilizing emotion and mental perceptions, but also enhancing social and emotional function. As Park and Paloutzian (2005) and Silberman (2005) argue, it is worth studying religion through the belief perspective by exploring in depth the religious meaning that each religious tradition has inherited.

\subsubsection{Prediction of Social Trust}

As with life satisfaction, the present study indicates that social trust is strongly predicted by social cynicism, mental health, and sense of community. Furthermore, none of the spirituality-related variables are shown to be predictive of social trust, thereby suggesting that the influence of spirituality on social trust is fully mediated through mental health and sense of community. The effect of spirituality related variables on social trust is also minor, compared to the other key predictor variables.

Several points can be drawn from the findings. Firstly, the present study provides empirically supports for significant association between sense of community and social trust. Welch et al. (2004) recognize that while some researchers look at societal factors or historical incidents for determinants of social trust, few empirical studies examine the effects of types of settings on social trust. As well as the few areas that were mentioned in their paper, namely ethnic diversity, scale and common values of the setting, the present study provides empirical support for a less researched area in trust research — sense of 
community — which the author views as worthy of further examination. Anderson (2006) also argues that community provides a more nuanced understanding of the settings which other political science theories cannot provide.

Secondly, although the association between social trust and mental health has been shown in the present study, the directionality between social trust and mental health is still under debate. While many recent studies in public health demonstrate the benefits of social trust on physical and mental health (De Silva, Huttly, Harpham, \& Kenward, 2007; H. M. Wang, et al., 2009; Yip, et al., 2007), Ryff and Keyes (1995) argue that a person with psychological well-being can develop trusting relationships with others. Therefore, the ordering of the two variables awaits further research.

Thirdly, the negative association between social cynicism and social trust shown in the present study accords with the findings of Leung and Bond (2004) that social cynicism is related to lower levels of cooperation with others and interpersonal trust.

Nevertheless, the absence of association between spirituality and social trust, when the other key predictors have been taken into account, seems to contradict Newton's (2004) argument that religious culture benefits trust. In the present study, as in the case of the life satisfaction variable, the influence of spirituality on social trust is mediated through the sense of community and mental health. Therefore, the present study suggests that spirituality still matters, but indirectly through the mediation of perceptions of belonging to a community and mental health. Moreover, as Newton (2001) argues, social trust is more the aggregate properties of communities, rather than individual members. It may be more beneficial to examine the relationship between social trust and spirituality from the religious community perspective, rather than from the individual spirituality perspective. In the qualitative part of the study, we will further examine the differential impact of social trust between the settings shown in the ANOVA section.

\subsubsection{Prediction of Civic participation}

For civic participation, the present study indicates that membership of affiliated organizations, fundamentalist belief, daily experience with God, and time spent in church activities together significantly predict civic participation. It should be noted, however, that the negative standardized regression coefficient of fundamentalist belief points to the 
negative effect of fundamentalist belief on civic participation, even though the influence of the other spirituality variables are positive.

Besides the direct effect of three spirituality-related variables on civic participation, two spirituality related variables (fundamentalist belief and years of attending church) also significantly contribute to the predicting membership of voluntary organizations, which is a major predictor of civic participation. The influence of spirituality on civic participation is also partially mediated through involvement in voluntary organizations. Nevertheless, similar to the direct path, the indirect effect of spirituality variables on civic participation through the mediating variable (voluntary organization membership) can be both positive and negative. Years of attending church have a positive influence on involvement in voluntary organizations, but fundamentalist belief apparently inhibits the positive effect of religion. Therefore, all these findings suggest that religion's influence on civic participation is complex because its influence can be both direct and indirect. Moreover, the influence can be both positive and negative.

The beneficial influence of religion on civic participation has been well illustrated in Wuthnow's award winning text Act of Compassion (1991), as well as much research around the globe (Beyerlein \& Chaves, 2003; Duriez, Luyten, Snauwaert, \& Hutsebaut, 2002; P. Y. Lam, 2002; Loveland, et al., 2005; J. Z. Park \& Smith, 2000; Patterson, 2004; Schwadel, 2005; Uslaner, 2002; Wuthnow, 1999). Nevertheless, research in this area also recognizes there are qualitative, rather than quantitative differences in the civic or political participations across religious traditions. While the conservative/ evangelical congregations tend to encourage members to participate in church related or affiliated voluntary works or organizations, members in the more liberal congregations are more comfortable to reach out to the society and work with people outside their faith community. The present study is further the corroboration in Asia, specifically, Hong Kong. Furthermore, the findings from the present study clearly show that fundamentalist religious belief inhibits participation in civic activities beyond the particular faith community. This illustrates what Wuthnow (1999) argues in his paper, that the mode and form of religious participation affects civic participation. The present study also demonstrates that the influence of religion on civic participation can also be both direct and indirect, and can be both positive and negative. Religion can be sweet and bitter because it both encourages and discourages civic participation. Religious people, who 
attend the church longer, spent more time in church activities, and experience more with God, tend to participate more in civic life, but there are also inhibiting religious influences on civic participation for those who embrace stronger fundamentalist belief.

\subsubsection{Overall Well-Being}

Apart from the individual pathway for each well-being variable, the path analysis of the present study provides us with a more holistic picture demonstrating the complex relationship between spirituality and well-being variables. It can be shown in three major ways.

Firstly, the present study indicates that spirituality related variables influence well-being variables, either directly or indirectly, or in a combined way, through the mediation of sense of community, mental health, and membership of voluntary organizations. This accords with the framework proposed at the very beginning of this thesis. However, it should be noted that the mechanism is quite dependent on the wellbeing measured. For example, regarding life satisfaction and social trust, the present study shows the influence of spirituality is only indirectly mediated through sense of community and mental health. However, regarding civic participation, the influence of spirituality is both direct and indirect. Moreover, while the experiential and participation side of spirituality has consistent positive influence on the studied outcome variables, the fundamentalism belief used in the present study was shown to have both enhancing (on mental health) and inhibiting (on civic participation) related to well-being, depending on the outcome variable. This accords with Koenig et al. (Koenig, et al., 2000) who argue that the relationship between spirituality and health is complex and not easy to articulate because not all religious beliefs and practices have a similar influence on health and wellbeing outcomes. Instead, it depends on the kind of well-being, the kind and dimension of spirituality. Levin and Chatters (1998) propose several pathways to describe how religion influences health and the latest research on the connection between religion and health explores the link between religion and health and well-being. All this indicates that the influence of religion on well-being can be richly diverse, if not intricate.

Secondly, the present study supports the multi-level understanding of well-being. Path analysis demonstrates that sense of community and social trust significantly affects life satisfaction. These findings accord with the emerging literature on the salutary effect 
of social capital on health and well-being (Baum, 1999; De Silva, et al., 2007; Helliwell \& Putnam, 2004; K. Mckenzie, Whitley, \& Weich, 2002; H. M. Wang, et al., 2009; Yip, et al., 2007). Although the definition and conceptualization of social capital is still in debate, there is a broad agreement in the literature on social capital that social capital is about the level of network membership, social trust, and sense of community, embedded in a social network (Baum, 1999; Lochner, Kawachi, \& Kennedy, 1999; K. Mckenzie, et al., 2002). Some believe that sense of community and social trust is an aggregate property and an indicator in the community about the quality of interpersonal or network relationships (Newton, 2001; Sarason, 1974), and they can be measured at the ecological rather than individual level. However, the results of the present study, using individual level data, support the notion that the well-being at the community level (feeling a sense of community and social trust) affects the well-being on the personal level (life satisfaction).

Thirdly, despite not being fully illustrated because of the limitation of the sample size, the path model for the entire sample seems not equally applicable to different settings. This relates to the issue of context in the study of religion (McGuire, 2002), well-being (Manderson, 2005), and their relationship (Lavric \& Flere, 2008). Many religious scholars argue that religion is socio-historically contingent, and thus cannot be understood in isolation from local context (Eliade, 1961/ 1999; McGuire, 2002; Smart, 1996, 1998). Manderson (2005) has a similar point of view regarding the study of wellbeing. She argues that well-being is not just in the subjective realm, but also "deeply embedded in and derives from society itself, socially produced and sustained by social structures and systems” (p. 13). Lavric and Flere (2008), in their study of the relationship between religiosity and psychological well-being in five different cultural environments, further corroborate this view and conclude that there is no culturally universal pattern (p. 164) in the relationship between spirituality and psychological well-being. All these researchers instead advocate examining the role of culture and the local context in dealing with the issue. The author of the present study is also convinced, from the finding of the present study, of the idea that the culture and context within the immediate settings matter and shape spirituality and well-being. Nevertheless, instead of becoming a relativist, the author believes that some cultural patterns and structural dimensions of the context are 
significant to well-being and worthy of more attention in later research into religion and well-being.

\subsection{What is left unanswered}

Although the survey findings provide some insight, these still do not fully answer some of the questions raised in the survey section. For example, while the influence of religious settings on life satisfaction is quite robust, why didn't this affect the members of the FDC church? While religious influence on social trust and civic participation is demonstrated in western studies, why is its influence only significant in one church, but not the other church in the present study? Although members in religious settings demonstrate a significantly higher level in spirituality and sense of community than their counterpart in the social centre, significant difference between the churches was also observed. The reasons for this are not very apparent. All these findings suggest that the effect of religion on well-being is not linear and simple, such as the more religious a person, the higher the level of well-being. Instead, the manifestation of well-being is closely related to the religious context. In the regression and path models, it was found that life satisfaction and social trust are related to sense of community towards the settings, implying the importance of the social context. Nevertheless, separate modelling analysis in each setting seems to suggest that each setting operates differently with different mechanisms. Therefore, apart from sense of community, what other contextual factors are relevant and able to enhance our understandings of the relation between religious context and well-being? A survey study is ill equipped to capture all the nuances of the settings and to fully understand the person in context. As such, in the following chapter, field study was used to make full use the qualitative approach and provide breadth and richness to the studied phenomena. 


\section{Chapter 11}

\section{Qualitative Descriptive Study}

To understand the person in context and further unpack the religious context are the goals of the qualitative study. Chapter Three discussed diversity of context (Trickett, 1996) - social structure, cultural environment, organization or immediate setting. Moreover, person and context are linked and cannot be easily separated. This relates to the critical issue of unit of analysis (Van der Veer, 2001). In this study, the immediate religious setting - congregation - will be focused on. As Berger and Neuhaus (1977) argue, congregation is close enough and not sporadic, so can provide people regular meetings to interact and influence lives. Congregation is the ideal place to understand the development of believers and witness the proximal processes that effect well-being (Bronfenbrenner, 1995). To understand religious settings, activity setting analysis (O' Donnell, Tharp, \& Wilson, 1993) is used to unpack the underlying mechanism and operation in each setting. The multiple-case approach is also employed to gain understanding of the similarities and differences across settings, so the relationship between religion and well-being can be revealed. In the following section, both the idea and rationale of activity setting analysis and the multiple-case approach are discussed.

\subsection{Activity Setting Analysis}

O’Donnell et al. (O' Donnell, et al., 1993) propose “activity settings” as the basic unit for understanding, analysing and intervening in communities. Activity setting is "the unit by which community and culture are propagated” (p. 504). There are similar concepts, such as behaviour settings (Barker, 1968), and microsystems (Bronfenbrenner, 1979), but activity setting is more powerful as it incorporates understanding of both the subjective experience and objective features of settings. O’Donnell et al. also argue that the different aspects of settings are interconnected and cannot be understood in separation from others. A closer examination of various facets of activity setting can reveal the complex interplay between characteristics within a setting and the social process in which people participate. As they argue, activity setting is the foundational interaction process 
by which “cognitions are developed, skills acquired, relationships formed, goals set, and activities carried out” (O' Donnell, et al., 1993).

To operationalise the concept, Gallimore, Goldenberg, and Weisner (1993) propose examining five intertwined dimensions: personnel, tasks, scripts, cultural values and beliefs, purpose or motives of the participants. Personnel concerns people available in activities, and the variations of the experience in a setting depend on the different combinations and interaction of people involved. Tasks are about the activities and tasks that people in the settings perform and are involved in. Scripts are about implicit rules or patterns of interaction governing people’s routine, everyday tasks and action. While cultural values and beliefs indicate what is embraced and emphasized in a setting, the purpose and motivation of participants unveil the underlying meaning that drives action and participation in the settings. Howecer, it should be noted that it is interaction, rather than the individual dimensions, that matters. According to O’Donnell et al. (1993), it is the "joint productive activity" (p. 505) that constitutes the heart of an activity setting and it is the synergy of various components in the settings that create the lasting impacts of the settings on people's development and well-being (Super \& Harkness, 1986). Activity setting analysis is useful to unpack the cultural influence of society on human development and is normally used to explore the relations between child development and cultural community (Farver, 1999; Gallimore, et al., 1993; Maynard, 2005). However, this approach can also be applied to other community settings (Gallimore, et al., 1993; O' Donnell, et al., 1993).

In the present study, the activity setting framework will be used because it shifts the focus of analysis from either individual persons or broader environment to persons in context. It considers both the person and environment as well as the objective and subjective. Moreover, the framework enables researchers to understand the complex interplay between characteristics in the settings. As a result, it can provide a richer picture of the settings. By using multiple case comparisons as the presentation, researchers can also make use of the strength of activity setting analysis to reveal the possible relationships between different religious settings and the specific types of well-being outcome discovered in the survey study. Although the claim of causation is not assessed on the basis of experimental or statistical models, multiple-case comparison can help 
identify the configurations of present or absent conditions that account for the occurrence or non-occurrence of specified states or outcomes (Lofland, 2006, p. 158).

\subsection{Multiple-case comparisons}

As Miles and Huberman (1994) argue, analysis and presentation cannot be totally separated. In the present study, analysis was done both in an inductive and deductive way. Using NVivo 2.0, the observational and interview data was grouped according to some emergent and significant themes, such as activity, artefact, relationship and language used. The author also searched the literature for a relevant framework and made sense of the findings collected. Finally, activity setting analysis (Gallimore, et al., 1993) was used to unpack the eco-cultural environments of the four studied churches, which the author believe is the most useful lens to understand the studied phenomenon. In presentation, four within-case analyses, following the activity setting framework, were carried out to unpack how each religious setting operated in different ways and to provide a rich account of each church. The cross-case analysis also provided the key dimensions to understand the studied settings and person in context. Two types of analyses are also valuable because, as Stake (2006) argues, within-case analysis provides experiential knowledge of each case (the particular), and multiple case comparison allows further exploration of the research question that penetrates the cases (the general). That relates to how religious setting enhances well-being, crucial to the present thesis. As a result, the cross-case activity setting analysis help to unveil how the cultural ethos and immediate specific religious setting can influence members’ spirituality and the specific dimension of well-being.

\subsubsection{Rationale and criticism}

As explained by Yin (2003), case study is a research strategy that allows the researchers "to understand complex social phenomenon" and "to retain the holistic and meaningful characteristic of real life events” (p. 2). It can help address the "how” and "why" questions. Case study methods make use of the multiple sources of evidence in the research process. This not only enables corroboration of findings by triangulation of data (Denzin, 1978), but also provides researchers with a wide range of tools that can overcome the limitations inherent in other forms of enquiry. For example, interviews 
allow researchers to explore the subjective meaning of the individual participants in the study, while participant observation permits researchers to enter the collective world and the experience shared by participants (Kral, 2007). Furthermore, documentary data can provide valuable resources to explore different facets of the same phenomenon. These different types of evidence provide a richer and holistic picture of the context under study.

Despite its strength, case study design has always been regarded as of low value, if not useless (Flyvbjerg, 2001; Yin, 2003). The often mentioned criticisms (Flyvbjerg, 2001; Merriam \& Merriam, 1998; Yin, 2003) include the notion that small N case study research cannot be generalized to create scientific theory. Case studies are also criticized for their bias and lack of validity and rigor. Furthermore, the detailed report of each case can be regarded as only anecdotal, if not judged to be too long to exhaust readers.

Nevertheless, as Yin (2003) argues, the generalization of the case study is not in a statistical sense, but in an analytical sense. Flyvbjerg (2001) further posits that even a single case (particularly a critical one) can be a powerful example to falsify existing theories and advance scientific development. In terms of rigor of the case study approach, Flyvbjerg argues that case study, like qualitative research, has its own criteria for rigor and validity that are no less strict, such as those mentioned in Lincoln and Guba (1985). Moreover, it is the detailed reporting of each case, Stake (2006) and Flyvbjerg (2001) argue, that constate its strength and merits, rather than the pitfalls, of case study. It is the particularity of the case and the richness of data -- the goal of case study -- which provides new understanding of the studied phenomenon.

\subsubsection{The Case and the Quintain}

As Stake (2006) notes, while a qualitative case study is about investigation of the experience of real situations, multi-case research normally starts with a research question (or quintain in Stake's term). According to his definition, a quintain is "an object or phenomenon or condition to be studied” (p. 6), which is what the researcher aims to understand when examining the similarities and differences across cases. In the present study, the cases are the four studied religious settings, while the quintain is our research question: how and why religious settings can enhance well-being. The four cases provide rich accounts of well-being related to each setting; comparison of the four cases, along 
with key features provided by activity setting analysis, enable the researcher to go beyond mere description, to explanatory accounts to answer the key research question.

\subsubsection{The issue of entry}

Stake (1995) suggests a “quiet entry” approach, and the researcher entered the setting basically in low profile. To conduct the survey, the researcher was introduced publicly to two churches (CHC, SCC) and to relevant people in the other two churches (FDC and EVC). The researcher, however, then attended church individually and alone. The introductory session in the four settings was welcoming, but later experience in different settings was quite different, which will be demonstrated in later chapters. It is worth noting that the first contrasting experience was felt in four churches in the first month. As recorded in the field memo, the human connection of each church and the church size was the first felt experience in the stage of entry.

After visiting for more than a month (basically 2 or 3 visits for each church), I find I still haven't made any acquaintance in EVC and CHC. I come and go without anyone noticing even though people are nice to each other. Fortunately, I see some familiar faces and have chatted with some people in FDC and SCC church. I think it is it related to the church size. For FDC and SCC church, with membership of less than 200, it is easier to remember others or even make friends. But for churches of over several hundred members (EVC and CHC, it is more difficult to make friends unless I join some of their small groups or more personal activities such as fellowship. Alternatively, I can start interviews in those churches so I know some people in more depth. In addition, I feel the congregations in SCC church are not very familiar with each other. One congregant, who attended the church for long time, but not frequently in recent years, do not know any congregant at all who had attended more than a year.

\subsubsection{Data collection method used}

The following data collection methods were used in the present study to take full advantage of the qualitative approach. All the data were collected, interrogated, and analysed in Chinese, while only the relevant materials were translated (by the researcher and cross-checked by a third party) and reported. 


\subsubsection{Participant Observation}

Participant observation has many strengths: access to events or groups that are not available otherwise; environmental conditions can be depicted; firsthand experience and “insider” perspective with participants and setting; unusual aspects can be identified during observation (Creswell, 2003; Yin, 1994). Participant observation is a good way to understand the phenomenon in the real life context and very suitable for use in studies of different cultural groups or organizations (Yin, 1994). Moreover, the method can uncover the daily routine of people in a systematic, but normally implicit, way. However, participant observation is not without its weaknesses. Yin (1994) and Creswell (2003) note that balancing both the participant and observer role is not easy: subjectivity and personal biases cannot be totally avoided, and good attending and observing skills are required. It is a challenge, particularly for an inexperienced researcher.

The student researcher ${ }^{10}$ participated in churches from July 2007 to March 2008. The researcher primarily attended Sunday service, but also participated in some other regular or ad hoc activities. Activities unique to specific church were attended, such as social concern activities at SCC, evangelical meetings at EVC, charismatic events at CHC, and evangelical outreach activity at FDC. The researcher also participated in some other general activities, like fellowship, prayer meetings, or meetings for the public. The activities attended by the researcher in each church are shown in Table 44.

Table 44: The activities participated in by the researcher in each setting

\begin{tabular}{lllll}
\hline & CHC & FDC & EVC & SCC \\
\hline Sunday Service & 13 & 14 & 15 & 15 \\
Fellowships & 2 & 1 & 1 & 2 \\
Evangelical activities & 0 & 2 & 4 & 2 \\
Religious oriented activities (e.g. & 3 & 3 & 4 & 2 \\
bible class, talk, prayer meeting) & & & & \\
Anniversary or celebrative activity & 1 & 1 & 1 & 2 \\
Other activities (e.g. social concern & 0 & 0 & 1 & 3 \\
and psycho-educational) & & & & \\
\hline
\end{tabular}

${ }^{10}$ A Hong Kong Chinese and active Christian 
Field notes were taken at the time of observation, where appropriate, and then expanded later. The physical setting, participating person and special happenings were documented, and reflections and insight drawn from the events were recorded as well.

\subsubsection{Semi-structured interviews and feedback sessions}

In the study period, a total of 23 interviews were conducted with members of four churches, from October 2007 to March 2008. The interviews were conducted in two phases: October-December 2007, and January- March 2008. In the first phase, two to four members of each church were interviewed and the interview finding were used to inform the survey analysis and formulate preliminary qualitative analyses. In the second phase of interview recruitment, further varieties of members were explored to enrich the understanding of each case. The recruitment of interview participants varied across religious settings, but generally was carried out in three ways. Firstly, with the help of parish workers, the researcher was introduced to church members so that the researcher could solicit for voluntary interview participants on the spot. While a list of potential interviewees was obtained, normally the senior members -- those who had attended the church for an extended period of time -- were chosen. Another way was snowball sampling (Neuman, 2006) in which the researcher was referred by the interviewees to other potential participants. Moreover, various sampling strategies were used according to the specific religious context, like negative variation -- trying to find people in different camps of the church -- and opportunistic sampling: that is, taking advantage of unexpected opportunities (Miles \& Huberman, 1994). The basic background information of the interviewees is outlined in Appendix 3 and the consent form and relevant documents can be found in Appendix 4 and 5. The questions asked in the interview were developed before the entry of the settings (see the interview guideline in Appendix 6 for details). The interviews were semi-structured, and the actual questions asked varied but the topics involved spiritual life, church life, legacy, or stories heard within the community, important moments in their spiritual and church life, and the impact and meaning their religious life gives to them. All interviews were audio-recorded and then transcribed by the researcher and another helper.

A feedback session on the preliminary survey finding was conducted in each of the participating churches (from January to February 2008), which one to five pastoral 
workers attended. Church pastors formed the primary audience for the researcher's presentations, and also provided valuable responses which were audio-recorded. Although these kinds of data are not the major data source of the present study, they provided the researcher with insights to understand the studied phenomena.

\subsubsection{Document analysis}

In the present study, church documents were also collected to corroborate or rectify the findings collected through other research methods. They not only included weekly brochures disseminated in the church, other church publications or anniversary reports, but also the pamphlets of other recommended activities organized by other affiliated organizations. Moreover, materials posted on the church website were also examined. On top of this, the researcher also analyzed other non-typical but relevant documents, like a seminary thesis studying one of the churches, and an autobiography of a pastor in charge one of the churches. These documents gave the researcher many ideas about the history or theology of the designated church that could not have been easily identified through observation. The researcher also gained valuable information about the routine operation of the church, like the church's regularly organized activities or prayer matters, which helped the researcher make sense of the everyday routine of the congregations (Schwartzman, 1993).

\subsubsection{Validity}

To ensure the validity, qualitative researchers have proposed different kinds of criteria and procedures (Creswell \& Miller, 2000; Denzin, 1978, 1989; Lincoln \& Guba, 1985; Merriam \& Merriam, 1998; Miles \& Huberman, 1994). Several procedures have been employed in the present study.

\subsubsection{Prolonged Engagement}

In the present study, field visits were organized, basically fortnightly, throughout eight months so that extended contact with the community could be attained. Prolonged engagement allows the researchers enough time to observe and make sense of the studied settings, and other sources of information or accounts and stories can also be heard in a natural way. In addition, as Yardley and Marks (2004) argue, prolonged engagement can 
mitigate the researcher's impact on the environment because members of the settings become habituated to the researcher's presence. Furthermore, by the repeated observation and extended contact, rapport could be built between the researcher and the members of the church so that information and invitations for interview can be more easily solicited.

\subsubsection{Triangulation}

A widely used technique for establishing validity in qualitative research is the triangulation method -- searching for convergence among multiple and different data sources, theories, methods or even investigators to form themes and understanding of a study (Creswell \& Miller, 2000; Denzin, 1978). Although it is recognized that triangulation method has its own limitation (Silverman, 2001), such as partial views of multiple sources and accounts that are partial and not necessarily able to give a more complete picture, it is still widely used to combine different sources of data or methods to make better sense of the other. As mentioned earlier, the present study employs multiple methods (observation, interviews, church documentation, and website information) to complement and offset the pitfalls inherent in specific kinds of research method. Furthermore, members of different churches were also carefully chosen, according to the ages, sex, the length of church attendance, so that varieties of account and deeper understanding of each church could be obtained (See Appendix 3 for details).

\subsubsection{Feedback sessions}

As mentioned earlier, feedback sessions were organized in each church which the respective pastor(s) attended. Although mainly survey findings were reported in the session, the researcher also shared with the pastors some observations regarding the church and some reflections on the research process. The pastors were able to express their different opinions or even correct the researcher's misunderstanding. Their opinions also informed analysis and interpretation at the later stage.

\subsubsection{Constant comparative method}

As suggested by Silverman and Seale (Silverman \& Seale, 2005), comparative method enables the qualitative researchers to test out provisional ideas, which can strengthen the validity of interpretation. In the present study, the multiple case 
comparison presentation allowed both the researcher and reader to compare the similarity and difference of each church to gain a deeper understanding of the relationship underlying religious setting and well-being.

\subsubsection{Reflexivity}

Various strategies have been employed to enhance the validity of data collection and analysis, and the researcher acknowledges that, despite trying his best to be objective in describing the congregation, subjectivity and bias cannot be avoided completely. The researcher further employs two measures of remedy. First, the description of each setting will be as detailed and comprehensive as possible so that the description of the settings and interpretation by researchers can be separated and identified more easily. In Chapter 12, the church settings will be described in detail, while the explanatory account of the relationship between religion and well-being will be follow in Chapter 13. Second, a reflexive account by the researcher is provided so that the researcher's personal biases can come under public scrutiny.

\subsection{Researcher's self reflective account}

Being a committed Christian for more than 20 years, the author has gone through the ups and down of the faith journey. Inspired and cultivated by a Christian teacher (with an evangelical or even fundamentalist background), the author became a Christian at 15 years old and settled in a medium-sized Methodist church in Hong Kong, with a mixture of evangelical and mainline tradition. The firm religious belief, intimate relationship with God and colourful church life gave the author good memories and personal growth in his teenage years, searching for meaning and identity. However, university life and some sporadic social teachings in the church and other Christian activities not only broadened his horizon towards faith and society, but also made him aware that continuously reflecting on one's belief, rather than just following others or tradition, is very important to developing faith if a person want to be faithful and truthful to the inner self. That is also the reason why he pursued religious study, after working for a few years, to understand religion and faith more objectively. The author realizes that he is not insulated from ethnocentrism or spiritual struggle in the time of ups and downs of his religious faith, but his striving to understand the other, and openness and respect for diversity, and 
greater understanding of the costs and benefits of religion, and self criticism, prevent a self centred view of the studied issue. As it is understood, in qualitative research, the researcher is the primary vehicle to collect data and interpretation, and it is worth mentioning here that about the author's previous church experience. The author has experience interacting with people from mainline, evangelical and fundamentalist churches, but limited connection with people from the charismatic church.

Although the researcher's committed Christian image may affect the responses of the participants (a few church members guessed the researcher was a theology student doing a study or taking a placement in the church), it is believed that the effect was minimum because of the habituation mentioned earlier (few church members felt surprised I was still attending the church several months later when they knew my researcher role). It may be argued that, in interviews, that participants may give socially desirable accounts, rather than a true picture of themselves, but participant observation in advance of the interview enabled the researcher to understand the interview in a more realistic way. The researcher also has 8 years experience of being a mentor at a college fellowship; here, college students often told him their problems or weaknesses even though they may have known that the author could have opinion different from them. The researcher's warm and sincere personality induced true responses from participants.

\subsection{Chapters ahead}

After providing an overview of the qualitative approach in Chapter 12, activity setting analysis was used as a framework to make sense of the qualitative data collected in each of four studied religious settings and to provide rich contextual information related to the studied churches. In Chapter 13, the author integrates the activity setting data with literature, to explain the significant finding shown in the survey section, and try to demonstrate how the activity setting of each congregation was able to influence its members' spirituality and, particularly, the dimension of well-being. 


\section{Chapter 12}

\section{The Activity Setting of Four churches}

To explore how religious settings is associated with differences in well-being, we used the activity setting framework to situate well-being within daily routine in four religious communities. In the first half of the chapter, each church, according to the five features suggested by the activity setting framework, is explored (Gallimore, et al., 1993). In the second half of the chapter, the five features will be further discussed in relation to well-being. Finally, a synthesis of key features in four settings is presented (see Table 45).

\subsection{Charismatic Church -- CHC}

$\mathrm{CHC}$ is a congregation with a charismatic background. It is a closely-tied church with a strong sense of community, now serving about 500 members. CHC emphasizes the power of Holy Spirit and encountering God explicitly and experientially, in which speaking in tongues is one of its distinguishing features. The survey indicates that $\mathrm{CHC}$ has significant and highest results in life satisfaction, while members also demonstrate a very high level of spirituality and sense of community.

\subsubsection{Personnel}

In $\mathrm{CHC}$, the leader has charisma and a gift from the Holy Spirit has often been mentioned and modelled. Members recall that the founder was a foreign missionary who learnt Chinese in just three months because his heart was with China. He started his missionary work in Hong Kong in the late 1960s, China in late 1970s. Because his heart was with China, the founder was hidden in a coffin to enter China, and even blacklisted by the Chinese government. He was also remembered by his followers as loving prayer, particularly prayer in tongues. A member (interviewee 6) recalled a time when he met the founder in the toilet, and he was still in prayer. Another member told a miraculous story about the founder in relation to his speaking in tongues:

Interviewee1: There is a night the (founding) pastor prayed in tongue. God moved him when he prays and he sees a vision. He sees many people going to hell and many people cry desperately. The other day he then knew that an earthquake 
happened in Taiwan and more than ten thousand people died. ....Thus, so often we know afterward what speech in tongues is exactly about.

Compared to the founder, the second generation leadership was noted as having strong commitment and pastoral care. Their charisma, as described by CHC members, is an important driving force of church dynamics. A CHC member commented that the new leader was a leader with charisma and who "can easily call hundreds response” and mobilize church members towards the same goal and "contribute actively, whether financially or physically”.

As regards relations with church leaders, the relationship between members is close and engaged, reflected in people's active participation in church organized activities, and also in strong feelings of belonging to the same community. A CHC member noted:

Interviewee1: it is not easy, for a church with four to five hundreds. It is not easy for all of us to know each other, because normally in a group of four to five hundreds people will not know each other. But I don't know why in our church, it seems that we will not be in the stage that we haven't talked to each other before. It is not uncommon to observe people pray for each other after the service. The supportive and selfless concern between members was also often recalled.

\subsubsection{Tasks}

In CHC, praise and worship is the primary activity, in which people's soul can be lifted high to even enter ecstasy. The critical role of praise and worship in the CHC community is stated clearly in their $30^{\text {th }}$ anniversary publication. The establishment and development of CHC can be traced back to an event in a Canadian Bible school in 1947 when a man who was tongue-tied suddenly fluently prophesied "the Lord would restore four aspects of truth that would culminate in the return of the Lord. These four aspects were unity, gifts of the Holy Spirit, praise and worship, and world evangelism (p.22).” The claiming of "restored truth", praise and worship in CHC, is reflected in the length of praise and worship at Sunday service (nearly one hour), and is also indicated by outward bodily movements, or even trance or ecstasy, of the leaders and congregants.

As well as worship, the cell group is also another crucial church activity in CHC because $\mathrm{CHC}$ is an expanding church with more than 500 people. To be able to care for 
members, the cells have been used since $1991^{11}$. There are now more than 50 cell groups in 10 parish districts. Each cell meets regularly in church or members' homes. Some meetings are religion-related while some are socially oriented. As well as cell meetings, cells will join together seasonally or yearly to organize district activities (such as picnics) so people from different cells can become familiarized and form networks.

Cells provide nurturing and supportive relationships between members, and $\mathrm{CHC}$ also organizes other activities from time to time, to meet the diverse needs and interests of members. The nature of the activities ranges from religious, evangelical, psychoeducational or even recreational. The teaching of the bible is improving, although some members commented that the teaching is not solid and systematic enough. In contrast, more religious programs are related to the power of the Holy Spirit (restored truth). The CHC often organizes revivalist meetings, faith healing sessions or prophecy meetings, particularly when affiliated guest speakers visit Hong Kong. Revivalist activities provide another channel for members to experience the presence of the Holy Spirit and enter the exotic realm is seldom experienced in daily life.

\subsubsection{Scripts}

\subsubsection{Free, lively, expressive and spontaneous worship}

Praise and worship play a crucial role in CHC activities, and worship that is free, lively, expressive and spontaneous is endorsed at CHC so that people can truly worship and praise in the Holy Spirit. During worship, lively music is important. People are invited to leave their seats and stand near the altar so they can be more freely involved in worship, and a small group of teenage dancers dressed colourfully will dance cheerfully, with ribbons and tambourines, in front of the whole congregation and near the altar.

In praise and worship, the worship team play a crucial role in affecting the mood of the session. Song leaders seem may not simply follow the order or schedule set in advance, but are open to change and to the intervention of the Holy Spirit. For example, leaders may extend prayer time when the congregation is very involved (reflected by the

\footnotetext{
${ }^{11}$ Instead of the fellowship format used in most churches in Hong Kong, which sometimes cater for 30-50 persons, cell groups in CHC contain around 10 people. A total of three up to seven cells will form in a district. Each cell group has a group leader, and each district has a district leader and district pastor who attend to spiritual needs more closely.
} 
continued speaking in tongues). Sometimes they suddenly kneel down and cry for the Lord's mercy and grace when they are moved to do so. This emphasis on spontaneity is verified by the $30^{\text {th }}$ anniversary publication (p. 23) in which the pastor-in-charge of the worship department encourages song leaders to be "sensitive to the guidance of the Holy Spirit and submit to the Holy Spirit's instruction."She notes a memorable event in which spontaneity and openness lead to guidance and instruction from the Holy Spirit.

Reporter: During the past 30 years, have you experienced something memorable? Pastor: A lot. In a recent worship meeting, we deliberately did not set the schedule of songs because we want the Holy Spirit to lead us in worship. That was a very special meeting: both the congregations and the worship team deeply experienced the presence of God.

\subsubsection{Tacit approval of exotic experiences}

In praise and worship, the song leaders and congregants are sometimes so absorbed they even enter ecstasy. The most distinguished outward behaviour is speech in tongues. The other outward behaviour when $\mathrm{CHC}$ members enter ecstasy includes falling down, laughing, dancing, coughing or trembling hands. CHC members attribute this kind of exotic experience to being filled by the Holy Spirit, as an interviewee explained:

Interviewee 3: Charismatic experience, I have. When you pray or sing, speaking in tongue does not necessarily happen instantly. But when you open yourself to God, you may be moved within your heart and then you will naturally start to cry and speak in tongue.

CHC not only accepts but sometimes even encourages members to cry and yearn for the Holy Spirit, by all means (powerful music, speaking in tongues, kneeling down or raising hands).

Song leader: Lord you not only live in the world. More importantly, you live in the life of your child and your people. Lord, we love to surrender our life to you. So speak to God, Lord, we love your presence in our life. Lord, live in our life. ......Lord, please present in our life and take hold of our life. (CHC 16-9-07 worship)

After the song leader cried out "Lord, we call your name! We call your name." Then many people started to speak in tongues, and some even sang in a language 
that the author didn't really understand. Some people knelt down as well. (CHC 16-9-07 worship)

\subsubsection{Using encouraging and engaging language}

If speaking in tongues is too exotic, the language used in $\mathrm{CHC}$ in other rational times is encouraging, inspiring and uplifting. For example, pastors often encourage congregants to greet and give encouragement towards each other, by saying: "Tell your brothers or sisters next to you 'you are loved and cherished by the heavenly Father." Congregants normally will follow. Even facing tension, the pastor can still give a gentle reminder by using language skilfully. For example, viewing the late arrival of the congregation, the pastor appreciated first the members' insistence of attending the church in cold weather before reminding them of the need for punctuality.

In addition to using encouraging words, CHC also uses many concise, rhyming and easily understood Chinese slogans or short phases to enhance memory of the main teachings of the church. For example, CHC uses the phase 生命見證隨時講，生命改變 認真爽 (ever ready to witness, so amazing to life change) to encourage congregants to share with others their blessing from God. When introducing an evangelical tool — 滿福 寶 (Fullblessing) the pastor quotes two funny Chinese couplets 我愛滿福寶，照著念就 好；三餐勤服用，好處不得了 (I love fullblessing, it is good to recite. Recite it three times a day, the blessing is uncountable).

Furthermore, various uses of the word "Amen” by song leaders, pastors and congregants is also worthy of note in CHC culture. In some instances, "Amen” is used at the end of a sentence, like "This year is the year of the Lord. Amen!" (then the congregation clap). In other instances, “Amen” is used in conjunction with hallelujah as an exclamation and praise to Lord. “......Hallelujah, Amen!” Nevertheless, “Amen” can also used by the congregants as a response to show encouragement and support to what has been said. For example, when a pastor prays “......to make Hong Kong belonging to the Lord", the congregants respond with "Amen” unanimously (CHC 16-9-07 worship field note). Last, but not least, another common usage of "Amen” in CHC is as a question, 
to elicit the congregants’ supporting “Amen”. Asking “Amen?” becomes a question: “is it?” Replying “Amen” means yes. For example:

Song leader: "Do we want to experience God today?”

Congregations: “Amen!”

Song leader: "Then we should worship him with all your strength. Amen?”

Congregations respond unanimously “Amen!” (CHC 16-9-07 worship).

Therefore, speaking in tongues, the use of positive language, easily understood slogans, and diverse notions of Amen, all help CHC create a spontaneous, dynamic, and engaged culture that encourages and enhances interaction between members.

\subsubsection{Cultural Values/beliefs}

The importance of the Holy Spirit, and being filled by the Holy Spirit, are crucial beliefs at CHC, as stated in "restored truths", and reflected in many activities, such as Encountering God Camp, healing and revival meetings. It is also demonstrated in members' talking intimately with God, in prayer, praise and worship sessions. Song leaders often call members to surrender before God in praise and worship (see p.172).

Besides emphasis on the Holy Spirit, living an abundantly rich life with your God is also highly emphasized by CHC, especially in 2007, because that was the church theme of the year. Having an abundantly rich life is reflected in church slogans, and throughout the year sermons focus on ways to attain it. CHC also organize large scale evangelical concerts during Christmas with the theme "A life with Happiness".

An enhancing and uplifting spirit can be felt upon entering CHC church on Sunday morning. Members in the serving team will greet you with smiles and an uplifting phase, "Welcome and Jesus blesses you”, and the church bulletin will be distributed. Before entering the main hall you will pass through a small but well decorated meeting place where there are boards depicting colourful church life. By the end of 2007, a very large and colourful board entitled "Thanksgiving blog” was placed near the entrance of the hall and many thanksgiving notes were posted on the board by members.

In the main hall, a huge, stainless steel cross stands in the front of the stage, with two large screens on both sides. Under the cross is a full set of musical instruments for praise and worship, including drums, keyboards, a piano, music stands, and amplifier. On the side wall, fresh and bright banners display the slogans of the church, creating a 
feeling of modernity. The church logo is a dove and flame, clearly related to the bible: Jesus' baptism and his baptizing people with the Holy Spirit and fire (Matthew: 3:11-16).

\subsubsection{Motivation}

Being filled by the Holy Spirit is, to many CHC members, related to the need to experience God in an explicit way. The exotic experience and outward behaviour is confirmation from God that He is present and in control, which is important particularly in mundane daily life. As one interviewee notes, encountering God normally brings outward behaviours, and experiencing God is the goal:

Interviewee 1: If you say we really want to experience God, that is correct. But does that mean encountering God is equal to the outward behaviours? It is not necessary. Why do we emphasise (encountering God) so much? It is because we have a strong belief that every time we come to church we have to see God. Seeing God means God talks to you. To my understanding, it means you have some changes in your life.

Nevertheless, being filled by the Holy Spirit is heavily emphasized by CHC, so that pursuing this kind of peak experience and subsequent outward behaviours may become seen as the ends (not the means) by some members. A CHC member observes: Interviewee 1: Being so close to God is indeed a special experience. But some people in churches with charismatic background endlessly pursue this kind of feeling, to an extent that it seems the Holy Spirit is not present in a person without trembling, or say, they attending every meeting with pastor with super powers. I think it is meaningless. They endlessly pursue a world-class pastor with power, as though chasing a superstar to get some blessing and experience from the superstar. I think it is meaningless.

As the founder of CHC also poignantly points out in the $35^{\text {th }}$ anniversary $\mathrm{CHC}$ video, the essence of charismatic experience is not an end in itself, but a means to an end, that is, to witness:

Pastor: Experiencing the filling of theHoly Spirit is not our major vision, even the praise and worship; nowadays many churches have better praise and worship practice than ours. The only one, and the most critical thing, is preaching the gospel and mission. What is charismatic experience for? To please God? To have 
people falling down, laughing and speaking in tongues? No, the charismatic experience is to enable and empower people to witness for God.

Being filled by the Holy Spirit, and being a witness to God, are two sides of a coin. Feeling the Holy Spirit is being witness to God, and it is necessary to experience the Holy Spirit from the outset. This can be seen in the CHC 35 anniversary video. The pastor encourages the congregation, by saying: "Yes, we really hope God can use our church to take on the vision of mission, but we know we first have to revive an energy -- to revive an energy to pray, an energy to preach the gospel, an energy to grow spiritually, so that we can take on the vision God gave us, to become a truly reviving church.”

\subsection{Social concern church -- SCC}

Compared to CHC, SCC is an older church that can be traced back to the late $19^{\text {th }}$ century. Like mainline churches in western countries, SCC is a small church with a stagnating, if not shrinking, membership. As with its western counterparts, SCC and its denomination have strong roles in the community, not only providing religious services, but also partnering with government to provide education or social service in Hong Kong. The survey findings demonstrate that SCC members have a significantly higher level of civic participation than their counterparts in other settings, either religious or nonreligious. In addition, SCC members also have significantly lower spirituality-related variables and a sense of community than other churches.

\subsubsection{Personnel}

Compared to the caring shepherd in CHC, the leader in SCC is an inspirational prophet. Pastor L was in charge of SCC for more than 10 years in the 1970s, introducing new practices and developing the unique identity that exists today. Initiatives in four areas helped develop uniqueness. First, the liturgy was transformed. Second, innovative lay member summer schools were run for five consecutive years, translating profound theology into something easily understood by lay people. Thirdly, the positioning of SCC as a church serving the local community explains why it developed varieties of community service and programs targeting local needs. Moreover, the concept of corporate ministry was introduced: pastors are responsible for the church ministry, but also play active roles in church development, collaborating with lay members. 
Compared to Pastor L's visionary leadership, Pastor T is a debatable pastor. His divorce not only agitates the SCC congregation, but also the Christian circle in Hong Kong, as reflected by a SCC member:

Interviewee3: Divorce is a taboo in church, not to mention the pastor who is having a divorce, which is the taboo in the taboo. How do they handle and view the issue?... How does the church understand divorce? Is it a must for those who are divorced to leave the church?... Are you expecting them to drift or wander outside? How can churches accept these people? Can those divorced get married again? These are all questions the church has to think about.

Like CHC, the participation of SCC members is also high, but the relationship between members is rather distant, if not tense. It was observed SCC members' involvement in church is vigorous, according with the SCC emphasis on corporate ministry, but also practical necessity. For example, it is observed that many young adults cannot attend the Sunday service because they need to take care of children's Sunday programs organized at the same time. Senior members also have many responsibilities in the church and because of great involvement in church ministry, there can be differences in opinion and perspectives. A senior SCC member notes:

Interviewee 5: Basically there is no problem when we are just learning and studying together, but conflicts arise when we work together. Sometimes, the conflict is meaningless. We lack the acceptance. We better not suspect others' motivation, since everyone works in different styles and has a different focus. If the decision is made reasonably at the time, we should not be to blame. Unfortunately, the divergence of different camps in the church has lasting negative impact on the church members and church development, which is awaiting reconciliation. The division of the church into different camps is symbolically reflected in two teenage fellowships which are aligning with different camps, and also in the hurt shared by SCC members. The internal conflict over the past decade has lead to many members leaving the church. Nevertheless, some signs of reconciliation were observed in the research period. Joint functions have been organized between two teenage fellowships, and people from the two major camps also study together, after inviting Pastor L back to SCC to deliver religious training. At end of 2007, the chapel was flooded because of a reptured water pipe, damaging the organ, electricity boxes, appliances, and other items. 
The cost was estimated at HK\$100,000 Hong Kong dollars. According to the church bulletin, people from both camps immediately went back to church to help clear the water. As a member noted, people leaving the church does not indicate a lack of a sense of belonging, but the converse. They leave the church because they love it too much, like a lover who breaks up in pain.

\subsubsection{Tasks}

Compared to CHC, programs organized by SCC are action-oriented. Their focus and concern is beyond the church, or even religious faith, such as welfare or social justice.

The social concern programs organized or co-organized by SCC in the research period include a people's forum and an exhibition about prejudice and discrimination faced by ethnic minorities in Hong Kong; a seminar on public consultation regarding the green paper on constitutional development in Hong Kong; an activity in the church to collect used clothes, stationery and money to donate to the needy in Philippines; a seminar about how the congregations in Hong Kong can show concern and support for “alternative economic" life in Hong Kong; and a sharing session inviting three asylum seekers from West Africa to voice their experience and difficulties in HK. Djembe and French lessons are also organized in the church so that asylum seekers who teach there can contribute and earn money for their efforts.

Nevertheless, compared to its splendid past, the social concern programs of SCC displayed in the period of the present study are ordinary. According to internal church documents, from 1985 to 2006, social concern programs or initiatives were the key components in SCC events. Programs can be divided into following major categories: educational or awareness raising, action and supportive (see Appendix 7 for examples).

However, regardless of its social concern, SCC has often been criticised by outsiders, and even some members, for their lack of biblical teaching. Some even labelled them heretical, for only being concerned with societal rather than spiritual matters. To be fair, instead of saying SCC has no concern with religious and biblical teaching, the teaching of SCC is sporadic and distant from the members. The SCC had a good reputation for lay members' summer schools in the 1970s. However, religious teaching today is far from satisfactory, reflected by several SCC members noting that most biblical 
and spiritual learning was from Christian bodies other than SCC. One member's comment on SCC teaching is insightful:

Interviewee 1: You cannot say there is no religious teaching. I attended children's Sunday school and youth fellowship and, until nowadays, we still have some 'so called' biblical teaching. The problem is teaching style.... SCC emphasizes the systematic study of bible, it is very academic. While it relates to life experience, it is more like studying at university - examining the historical background of scripture, and how and why something is said.. Even when it may relate to people's lives, it is rather removed. For example, when discussing the relevance of the bible to life, it tends not to relate to what we feel, but to social problems in society.

\subsubsection{Script}

Compared to the expressive style in CHC, emotional expression normally gives way to concern beyond oneself. It is manifested in Sunday service, whether in prayer, sermon or benediction. For example, every Sunday there is a prayer time inviting people to share in public what can be prayed together. During the 13 visits in the research period, only one prayer was for members' needs (led by the new pastor who prayed for a member who was to undergo major surgery). The other prayers raised publicly were all about societal problems, such as the demonstration in Burma, exploited building workers in Hong Kong, or hostage taking in Afghanistan. After the prayer time shared openly, a passage of prayer, prepared by the congregants, is read altogether. Although the theme may vary, concern about societal issues will be mentioned in the prayer session. During the research period, two prayers primarily focus on political issues, including constitutional development and democracy in Hong Kong. Even in individually oriented prayers, the passage will draw the members to societal problems before asking for the Lord's repentance. The following dialogue is extracted from a prayer on a festival day: Service leader: The ruling Father, with the advent of the Lunar New Year holiday, marking the Year of the Pig is over. We are accustomed to say congratulations on the words, such as: Kung Hei Fat Choy, have a healthy and pleasing life. But in this festive season, do we also say hello to you and do we still remember your great love to us? 
Congregants: Lord, have mercy on us. (x2)

Service leader: Almighty Father, the recent arrest of a suspect by the police regarding the nude posting of a celebrity ${ }^{12}$ seems like disclosing the event when the offenders deserve punishment, but the event is just the tip of the iceberg. Moreover, the event reveals people's disrespect of the others' privacy and disregard of others' rights. Lord, we are all sinners and ask for your forgiveness so that we can learn from the lesson and bring no more hurt to others by satisfying our desire.

Congregants: Lord, have mercy on us. (x2)

Service leader: Gracious Father, when most of us in Hong Kong can enjoy the festival with our family, our Chinese compatriots in Guangzhou cannot return home for festival because of heavy snow. They not only cannot enjoy the reunion with family members, but also suffer from hunger and cold. Some even lose their lives because of accidents. Lord, we pray for the commuters who are still waiting to return to their home towns, and we pray in Jesus name.

Moreover, apart from prayer, sermons at SCC do not lack a societal message, even though religious teaching is still primary. For example, in the studied period, a Christian legislative councillor was invited to deliver a sermon about the minimum wage. Speakers or pastors, active in both the Christian circle and in civil society, have been invited to give sermons. In addition, before the end of services, pastors conclude by saying that "Be prepared to go serve humanity”. It is a normal and routine phase, but its impact is lasting.

\subsubsection{Cultural Values/beliefs}

\subsubsection{Social Concern and Action Orientation}

Compared to the uplifting spirit encouraged by $\mathrm{CHC}$, the religious worldview inherited from the SCC tradition is action and socially oriented. In other words, religion is not a privatized matter, but expressed outwardly through practice, including action in

\footnotetext{
${ }^{12}$ The celebrity sex-photo scandal, of February 2007, concerning the release on the internet of nude photographs of several Hong Kong movie stars, raised discussion within society. Many (including the other three studied churches) mainly focused on the moral dimensions of the issue - whether the celebrities were morally wrong. However, others (including SCC), were more concerned about protection of, and respect for, personal privacy.
} 
the community or even the political realm. This is reflected in the varieties of organized socially oriented activities (discussed previously), and also by a legacy circulated in SCC: the persistence of seven women in serving a patient with paralytic stroke.

Interviewee 3: An always mentioned story is one involving Pastor L leading a group of seven women.. There was a person who lived in that block which you can see nowadays, even though no one lives there any longer. That person had a stroke and you know how difficult it is for a person like this to live in those sevenfloor-resettlement ${ }^{13}$ blocks without help. So, Pastor L mobilized seven women (and not one of them is still alive), to do the man a favour. For example, helping the man boil water in the morning, cooking him rice or helping him buy something. Each woman was responsible for one day only. These kinds of service are great and the idea was later developed into the community nursing practice. SCC emphasizes this very much and that explains why some elders who do social service also regard it as a serving opportunity because this is what they can do to the least of the brothers. They are not proud of these, but they will talk about this. Besides this story, the fact that the SCC is socially concerned and action orientation is also shown in the built environment. For example, Chinese Buddhist style couplets in the main entrance of the chapel say: 深情種善根, 愛意渡眾生 (Breed good endowment with affection and save the masses of mankind with love). In one of the function rooms there is a world map saying 天下為公 (World is for all mankind). Upon entering the main hall, written on the wall on both sides of the main entrance, are four words in Chinese and Greek: preaching, teaching, fellowship and service, which are the four basic functions of the churches, according to a senior SCC member. Inside the hall, 14 medium sized glass paintings hang on both sides of the sanctuary, depicting the stations of the cross of Jesus Christ. Towering above the altar stands a huge cross with four pictures placed at each quarter (which is also the church logo). One of pictures depicts two hands embracing Hong Kong Victoria Harbor (representing Hong Kong). As explained by a SCC senior leader, the picture symbolizes the hope that the congregation can serve the community and society with their efforts. In addition, since SCC insists on

\footnotetext{
${ }^{13}$ In 1960-1970s, the settlement house was built on seven floors, without a lift or facilities for people with disabilities. In addition, the toilet and kitchen is exposed in the public area.
} 
having no air conditioning, fans are mounted on the wall (on top of those glass paintings) and floor type fans are placed all around the sanctuary. In the summer, there may be more than 10 fans whirling when you are having a quiet and serious Sunday service. It is not unfair to say that the SCC sanctuary looks shabby rather than grand.

The emphasis on action in SCC is closely related to their understanding of faith and what is meant by love.

Interviewee 4: If you are too emotional, such as saying Jesus love you and I love you, I also will not accept...... because I regard it too casual. You say you love me in this moment, but when I ask you to borrow money the next moment, will you lend it to me? Will you really act out? Do you know my point? If you want to say you really love, I will ask you how you show the love. That means it is too vague (if it is just saying). But if you show your love through service, it is more real. For example, I prepare a meal for you. This is an expression of love. But if you just say I love you even though I know you are hungry, then it is meaningless.

Nevertheless, there are still variations in the understanding of action. While some understand action as serving and caring for others, others take social action or even political participation as the expression. What is common is faith in action.

\subsubsection{Openness and diversity}

The second critical perspective in SCC religious belief is its openness and acceptance of diversity. This is shown by welcoming people from marginalized backgrounds. For example, SCC has hosted $\mathrm{WTO}^{14}$ peasant protesters who were detained by the government, and some dissidents from China who were waiting to go overseas. Two members also remember that SCC had members who were ex-prisoners or beggars. In addition, SCC's acceptance of diversity is evidenced by their permissiveness to some sensitive, if not taboo, topics. Just like the divorce of the Pastor T mentioned earlier, SCC allows the space to think and discuss. They even organized or co-organized seminars on homosexuality, AIDS or understanding other religions.

\footnotetext{
${ }^{14}$ The Sixth WTO Ministerial conference was held in Hong Kong from 13-18 December 2005.
} 


\subsubsection{Motivation}

As explained earlier, SCC members' action oriented faith is closely related to their understanding of love, which is deeply rooted in their understanding of their religious faith. In their understanding, an abundant life is a life for others, like what Jesus did. Therefore, it is not difficult to understand why SCC accepts marginalized people. In their commitment they realize their true religious faith.

Interviewee 4: Since I always feel that all our gifts is from God. It is more blessed to give than to receive. In giving we offer ourselves to others and this is the example set by Jesus. Jesus was to offer himself to human. In giving we can see the abundance of life. Do not reduce our life to ourselves only. When we live for the others, we will find our life more meaningful.

In term of SCC members' openness towards diversity or even taboo issues in religious faith, an underlying belief is that religious faith is not as simple as many Christians assume. That means that there are no fixed but rather varieties of ways to understand and pursue religious faith. In their understanding, religion is so rich that people cannot fully understand it. What people can do is to humbly pursue faith.

Interviewee 3: Then we have to find and pursue (the faith) endlessly and open ourselves. This is also what SCC gave me-- to face, to challenge and to be open. As a consequence, SCC members are reluctant to accept certain typical models of Christian or religious faith or practices. That also explains why some members hesitate in their attitude towards evangelism.

Interviewee 1: Many people say preaching is very important in religious faith, but I feel it is not as simple as organizing large scale evangelical meeting. It is because we also know history that preaching religion also bring a lot of problems. I don't think it is necessary to preach religious faith in such an intentional way. Rather, it is better for someone to believe if they are interested in religion because of what you have done. That is better and more enduring.

Nevertheless, as some members argue, because of an overemphasis on social concerns and diversity, the essence of religious faith has given way to the human mind and personal will. That also explains why the estrangement between the two camps in the church cannot be healed for some time. 


\subsection{Fundamentalist Church -- FDC}

In contrast to the other congregations, FDC is the only branch seeding church which started in the 1990s. With a strong passion for street evangelism, FDC and its mother and sister churches have roots in the Brethren movement tradition and have a conservative, if not fundamentalist, spiritual outlook. As a branch seeding church, FDC has developed its unique type of spirituality which is more open. The survey findings show that FDC members demonstrate a significantly higher result in social trust than their social centre counterparts. They also have a very high level of spirituality and sense of community in relation to their church. However, FDC members did not demonstrate the generally positive religious influence on life satisfaction shown in other studies, or compared to three other religious settings.

\subsubsection{Personnel}

The leaders and members of FDC developed a unique and egalitarian relationship with each other. This can be shown in several ways. Firstly, an interesting practice in FDC is that the FDC members will not use the term "pastor”, “chaplain”, "minister” or "Reverend" to name those who serve in the church. Instead, they refer to senior members or those who serve in the church as brothers or sisters, thereby saying Brother $\mathrm{X}$ or Sister Y, instead of Pastor X or Rev. Y. Secondly, lay-leaders take a lot of responsibilities, even with the sacrament. This is explained in a church document: "In all kinds of sacrament, all brothers who are spiritual and well respected can take charge of it. Conversely, any pastor or elder who is not spiritual is not encouraged to do so.” Thirdly, although the leadership of the FDC's mothers church --PEC -- has been noted for its strict manner, the new generation of FDC leadership has a closer relationship with members.

As well as close and mentoring relationships between member and leaders, the relationship between members is close, sincere and supportive, clearly demonstrated in this member's account:

Interviewee 5: Regarding the caring of brothers and sisters, there is a lot of love expressed in real terms. In fact all these is influencing me, because I think not much love is in this world, or at least not as sincere as shown among us. They (the 
other members) will blame you if you are wrong, but will support you if you are right. But the people outsides may use a different lens or different attitude.

\subsubsection{Tasks}

In contrast to $\mathrm{CHC}$ and SCC, the programs provided by the FDC church are religious and rational. Sunday activities, particularly the breaking of bread (communion service), are very important. The primacy of breaking the bread is not only reflected in it being held weekly (rather than practiced monthly by other churches in the present study), but is also demonstrated by serious handling of the activity. The breaking of bread service in FDC is only open to brothers and sisters baptised in Christ. The church guidelines even mention that those Christians from another church should be examined clearly regarding their orthodox religious background before being allowed to join the activity. The sacred status of breaking the bread in FDC members' lives is spread throughout church documents, but also shown in members' accounts. A pastor in the FDC mother church, when teaching about the communion service, recalls her being taught about the critical role of communion service taking precedence over one's wedding date. Several interviewed members, when recalling times they could not attend the communion service, showed their sadness, if not regret, at missing the communion service.

Besides some regular activities, such as fellowship, there are still other programs standing out among FDC organized activities. Two of these are evangelical type activities, and family type activities. In terms of the evangelical activities, FDC not only hosts evangelical meetings regularly on Sunday mornings for non-believers, but also actively organizes evangelical outreaches monthly in the nearby street or area so as to spread the gospel. The passion for preaching the gospel directly and in person is the founding element of its mother church, documented in historical study of the denomination of FDC.

With respect to the family type activities, FDC have several kinds of activities that aim to build relationship between the members. For example, they regularly have a "family gathering function" for congregations to meet at members' homes to pray or share on the weekday. "Member sharing sessions" are also organized every month in the church, with special themes, so that members from different fellowships can communicate with each other about church issues and religious life they are interested in. On top of that, FDC organizes church cleaning days every several months so that every 
member of the church can actually take part and share responsibility for the church they belong to. Although it has become a bit ritualistic nowadays, it is a tradition with historical grounding.

\subsubsection{Script}

Compared to Sunday service at SCC, which is rather formal and structured into four sections, Preparation, Confession, Holy Word and Offering, the format of FDC is very simple: hymns, sermon/ breaking of bread, and announcement. In other words, no liturgy is concerned. While Jesus' crucifixion is highlighted in the breaking of bread session, God's word is the focus in Sunday service. The simple program of church activities is documented in the "Church spiritual guideline" document:

2.1 We have no fixed procedure in our activities, but the procedure set by the responsible persons who are guided by the Holy Spirit.

2.8 We have no creed, Lord's prayer, benediction or any call to worship in our activities.

This kind of practice accords with emphasis on the primacy of scripture over everything else. Despite simple procedures in church activities, the needs of members are remembered, even by name. For example, in the church announcement (titled "family report”), important church matters are publicized and prayed about together. They include church events, but also members’ updates that invite others' concern and attention. A typical example is the following:

Prayer matter on 15/3/2008

1. Please remember the mission camp on 21-22/3, the evangelical meeting for parents on 23/2, and the evangelical meeting organized by XXX fellowship on $28 / 3$.

2. It is the peak period of influenza, pay attention to personal hygiene and may God bless all of us physically healthy.

Members' news

1. After the surgery, the physical health of Sister $X$ is ordinary, and sometimes with emotion. Please remember the psycho-spiritual need of the sister.

2. Sister $Y$ will give birth to her baby on $28 / 3$, please remember the baby and health of sister. 
In addition, simple, direct, face to face interaction between the members after services is worth noting. There are separate sessions of activities on Sunday (the breaking of bread, Sunday service, gospel service or Sunday bible class). In between the sessions, FDC members have time to interact, chat and share. Moreover, because of limited space in church, the hall, which can house nearly 200 people and be divided into several small function rooms by the use of the folding doors, can be rearranged to serve different purposes. FDC members will often be casually called in, either on Saturday or Sunday, to help arrange the chairs. As such, church as a family is encouraged. From church members' interviews, it is known that a lot of other caring or recreational activities, including visiting the sick, hiking, gathering, shopping, or even travelling, were organized through this kind of short and plain interaction.

\subsubsection{Cultural Value/ belief}

FDC is undergoing change. With close affiliation with its mother church, FDC has been greatly influenced by its more fundamentalist belief and culture. As a branch seeding church, it also develops its unique identity which is more open towards change and family type.

\subsubsection{Truth over everything}

In the "Church spiritual guideline" document, the supreme primacy of religious matters over all things is clearly stated in the guideline number one - overall principle.

1.1 All the activities, services, meetings, systems, rituals, affairs, directions, messages, gospel, administration, external affair, thought or appointment, should be focused on God and the soul of people. This means it should be focused on the things with eternal values, at the direction of heaven, moved by the Holy Spirit, at the truth of the bible. This is so-called "spiritual route". We take that at heart, as the center, as the backbone for the principles of goal, as the criterion. Other aspects, such as people's physical, psychological, knowledge and wisdom of people, the decoration, music, beauty, the excitement of many people, organizations management, the spirit of democracy, talent and intellectual skills, money and other material ... and so on, are of secondary importance. 
The emphasis on religious matters over all the earthly desire is reflected in church documents, and also in built environment and members' accounts of church activity. For example, no cross, no pictures, and no icons are hung up; only a banner describing the church theme is displayed above the pulpit. At the annual summer camp, the emphasis is on religious teaching and revival of spiritual life that should not be compromised by human weakness. A young member remembers the harsh demands of summer camp:

Interviewee 3: For example in the summer camp, we have to wake up at 6 in the morning and then listen to three sermons. Actually the summer camp is always like this, only sleeping, eating and sermon. Basically I am ok to this high intensity, but it does not consider human weaknes. We can get really tired and find it difficult to concentrate. To me, it is inhuman, but I still appreciate their seriousness.

The emphasis on religious matters over everything not only downgrades earthly desire, but also dichotomizes religious things as good and earthly desire as bad. Everything is black and white, clear-cut and simple. Therefore it is not difficult to find, from interviewees' accounts, feelings of guilt when religious ideals are compromised for earthly desire, such as living comfortably, earning money, or having sexual feelings.

\subsubsection{Church under change}

Having said that biblical truth is clear-cut and cannot be compromised, the commitment of FDC church members is not absolute, even ambivalent. The ambivalence of FDC members towards their own tradition can be shown in many ways, and one is the overt behaviour permissible in their church. For example, three FDC members mentioned that the dress of the younger generation in FDC had become looser and more casual. The decline of strictness in FDC is not only limited to the permissiveness of certain behaviour, but is also reflected in the commitment required of a believer in their religious faith. Two members also stated that fellow members in the past were more passionate in their faith-seeking and preaching the gospel, even dying for Christ, but that is not the case nowadays. A deacon reflects:

Interviewee 5: In the past, we emphasized personal devotion and reading bible. We put a lot of emphasis on the spiritual relationship with God. But it is weaker now. Maybe the pastoral workers understand that it is difficult, if not impossible, to push the 
newcomer in this way...... When I was baptised before, I remembered we were called to die for God. We were asked whether we had thought of this and whether we were willing to do so. I felt it was right and necessary when I was baptized more than 10 years before. But (now) when I see some people to be baptized, I ask the same but, unfortunately, one person finally left the church because of feeling hurt when being asked to die for God...... Then I understand, we cannot just pass on something that we took for granted, because the extent to bear, or the value, or the point of view, of people has changed.

In addition, the difference between the FDC and its mother church can be shown by two incidents. The first relates to a pastor's openness towards new ideas and practices. Instead of using the traditional top down approach practised in the mother church, a pastor in FDC trusted and allowed the youth to organize programs on their own, resulting in support from the congregation. The second event is about the reduced obedience of congregations towards the leadership in a significant church event -relocation ${ }^{15}$. Because of an increase in church members, FDC decided to buy another building to accommodate the increasing number of people. However, as the area of relocation was quite far from the original place, many FDC members were strongly against the initiative, thereby creating tension between the leadership and members of the church. Finally, the leadership of the church compromised and cancelled the relocation plan. Having said that, although FDC members are no longer so apt to embrace traditional belief passed down, the church as their family and spiritual home is still indicated explicitly in many members' accounts of their church, even in the event of church relocation.

\subsubsection{Motivation}

FDC members' use dualistic thinking and emphasise religious truth above everything to show their willingness to sacrifice for the truth, whatever the cost. Here, a member notes how she tried to overcome bodily discomfort to attend the church summer camp, which is normally not well-equipped:

Interviewee 2: In the past I also didn't like the summer camp. Why? It is because I seek the ease and comfort and I cannot sleep very well in the camp..... the other

\footnotetext{
${ }^{15}$ In church documents applying to all PEC churches, it is stated clearly that congregations need to observe and obey senior members who have spiritual power.
} 
is about the toilet and bathing. I only will go when the place is nice. That is why I always struggle every year......But every time I go I also feel it is not at waste of time and it is worth going ......Last summer......I needed take medicine at night to sleep, but I feel it is worthwhile to overcome. Since I was young, I like comfort, but now I know the proper way of thinking: God not worthy of me to do it for him? Not everybody who is ready to sacrifice for the truth. Standing at the crossroads, identifying with the religious ideal, but understanding the difficulties, is the norm. Although the ambivalence may be related to unwillingness to sacrifice, it may also be about uncertainty towards change and new perspectives, as alternative to make sense of religious faith are sought, particularly when traditional notions are not fully applicable. This ambivalence is evident in the following conversation initiated by a FDC member at the end of the interview session.

Interviewee 6: Our church life. It is a bit long, how do you think? Sometimes it makes brothers and sisters very tired.

I: Have you reflected?

T: But we also feel.... Reflect? Whom to reflect?

I: The leader or elder?

T: Sometimes I think, can you pay a little for God? If you suggest me to cut this and cut that, that is not good. They are good, but added up, it takes much time.. In addition to both the willingness and ambivalence to sacrifice for truth, guilty feelings are also found in the FDC member interviews; they feel they are not living to the standards and not worthy of God's abundant love. However, while they are fully aware of their own weakness and sinful nature, they are also grateful for God's acceptance and grace given their own imperfections.

Interviewee 1: In terms of the things that I want and the things that I am worried about,, God not only protect me from worry, but also provide for me in every possible way. In breaking bread yesterday, brothers shared how Jesus died for us and I felt ashamed and torn. Once I have little difficulties, I am like the Israelis and become weak and have no confidence. Can you see how, how, how unworthy I am? I really do not deserve to be sons and daughters of God, but God lets me see He loves me so much. That is what these three years are about,, they are indelible. 


\subsection{Evangelical Church -- EVC}

EVC church was founded in the 1950s. With its huge size of more than 5,000 members, EVC is one of the strongest representatives of the evangelical church in Hong Kong, providing a wide range of religious and evangelical activities and programs. With a diverse demography, EVC now has four separate church buildings in the same region and passers-by are not uncommon. It is not an exaggeration to say that EVC is more like a small community rather than a closely tied congregation. The survey findings demonstrate that EVC members have a higher and significant level of life satisfaction than their social centre counterparts. Like CHC and FDC, EVC members also demonstrate a very high level of all kinds of spirituality-related variables. However, members' social trust and sense of community towards the church are much lower.

\subsubsection{Personnel}

While Pastor $\mathrm{K}$ is the pastor in chief, Pastor $\mathrm{W}$ is the pastor responsible for about 50 pastoral staffs and 5000 congregants. The legacy they leave members is humble in character. The EVC $55^{\text {th }}$ anniversary publication is full of members recalling how Pastor $\mathrm{K}$, with prestigious qualifications, started work in EVC with very low pay; how he committed and contributed to the church, even by sharing his home so that more members can be housed on Sunday. In contrast to the quiet, calm and humble servant image of Pastor K, Pastor $\mathrm{W}$ is a gentle, stable and dignified person who can master the numerous affairs of the church. Besides being a Pastor in chief of a church with more than 5000 members, Pastor $\mathrm{W}$ also plays an important role in the denomination and some evangelical organizations, and he is a famous speaker in Hong Kong. However, as described in the EVC $55^{\text {th }}$ anniversary publication, Pastor $\mathrm{W}$ still attends closely to the needs of pastoral workers and members of the church amid his busy schedule (p.68-70). The leadership, characterized as both diligent and humble, has gained a favourable reputation across the congregations. Nevertheless, the pastors of EVC will show their authority and power in times when the biblical teachings are violated or challenged. For example, a member remembered a gay person argued fiercely and in public with the pastor of EVC in church, which finally led to an unpleasant event. 
EVC pastors are nearly perfect role models as humble servants, This means that, firstly, many members are obedient and conflicts are rare, even when church members may disagree with church leaders. A member observed:

Interviewee 3: EVC (members) are well-behaved. EVC has its unique culture or tradition ......There is less conflict. Certainly there will be conflict when there are people, but it is relatively rare and harmonious (in EVC). Pastor K and Pastor W are approved of by brothers and sisters. They are very perfect models and lead the church well. That is why few object to what they say. This is my observation. Secondly, taking responsibilities in church is not easy unless people are committed and have good character and reputation. A large church requires much manpower and it is careful how it chooses those who will not depart from orthodox religious faith. Some members also recalled experiences of being neglected, or even rejected, when they wanted to contribute themselves to church.

While it is good for the members to take serving the church seriously, a drawback is that loafing is not uncommon. In addition, feeling lost or lacking a sense of community was often mentioned because of the lack of participatory roles.

\subsubsection{Tasks}

EVC provides very comprehensive religious services and education to which no other church in Hong Kong can compare. The Sunday bible school of EVC is exceptional. About 30 classes, from introductory to intermediate or advanced level, are offered every three months. Some of the classes are even taught by famous lecturers or professors working in the seminary in Hong Kong.

Sunday Service is another critical activity at EVC. Church documents suggest the centre of worship is God, and people (even passers-by) attend EVC service because of its strong teaching and preaching of God's word, especially by famous speakers.

In addition to typical religious teaching, EVC also has specially designed activities for target groups. For example, men’s groups are organized for male congregants, in view of declining numbers. Chastity agreement meetings are organized for youth to educate them in moral and Christian ethics about sex. Some emotional support groups or talks are organized for members with special needs, such as patients or family members facing cancer, terminal illness or emotional problems. For those who 
have an interest in music, EVC also provides both training and performing opportunities. If someone is concerned about the society, a study group and some irregular talks are organized, although the topics are mainly around moral ethical issues, like homosexuality, or gambling, concerning Hong Kong in general.

Nonetheless, compared to its evangelical program, these activities are are quite ordinary. In the study period from July 2007 to Mar 2008, there were nearly 10 large scale evangelical meetings organized by EVC, not mentioning those held in EVC, but organized by other organizations. In addition, bible classes, movie sharing sessions or gospel lunch programs run continuously throughout the year, whether on weekends or week days. Furthermore, EVC also organizes innovative programs to appeal the outsiders. For example, music performances are organized by the choir or musicians of the church. Basketball competitions are coordinated for the youth who can bring friends or nonbelievers to the church. They even organize a few days gospel journey to mainland China during the Mid-Autumn festival [Chinese festival] so that members can invite relatives to the Christian circle in a non-threatening way.

After becoming a Christian, new converts or new members of EVC can find numerous activities to strengthen their faith or deepen their biblical knowledge. In the meantime, they will be introduced to suitable fellowships or groups so that they can enjoy a deeper social life in the church. Welcoming tea gatherings or spiritual birthday celebrations are regularly run for new converts or new members so that more information about the church can be introduced. Varieties of small groups on different themes are also available for the new members who have joined EVC for certain period of time, but still have not found appropriate fellowship.

For those who are stronger in faith, there are wide ranges of activities or training programs to equip congregants to preach the gospel or to serve in the church. For example, there are training programs, lasting for a year or two, to prepare congregants spiritually and practically to take care of new converts, or to be mentors of fellowship, or to serve in a different division of the church.

As well as local evangelism, EVC is dedicated to a global mission. It not only coordinates regular mission sharing sessions, inviting missionaries to share their cultural and overseas experiences, but also structures overseas mission trips so that the members can get first hand experience of global missions. In the study period, EVC organized 
mission trips to several Chinese provinces with which they had connections; the mission fields introduced in EVC activities are not limited to mainland China, but also include Cambodia, Europe, Arabia, and Africa.

In short, the EVC provides a wide range of activities or programs to cater for the needs of members, across a wide age range, or in different stages of religious faith. It is not an exaggeration to say that EVC strives to attend to a variety of needs - from those of non-believers to those able to preach the gospel competently.

\subsubsection{Script}

Compared to the three other studied churches, EVC is a large scale congregation in which formal rule and structure is expected. It can be shown not only in the hierarchical organization, but also explicitly in the long queues before the service and the formal and systematic, if not hierarchical, procedures.

\subsubsection{The long queue before the service}

At EVC, there are 10 services spreading through Saturday afternoon to Sunday evening, catering for different age groups and populations. Taking Sunday morning as an example, there are four Sunday services provided in the main hall of EVC, as well as services that are held in other church buildings and designed for people from a Mandarin or English speaking background. Due to the packed schedule, and to be able smoothly operate the services, time management and crowd control is very important in EVC.

In EVC, there is a practice that people who are more than 20 minutes late are not allowed to attend the service, so church members are punctual. Moreover, many people come earlier to obtain a better position in the service. However, because of the packed services, people who come earlier must wait outside to wait for the earlier service to finish. It is intriguing to note that every Sunday morning, about 15 to 20 minutes before the service commences, several very long queues ${ }^{16}$ of people, from 50 to 100 meters long, form in an orderly manner outside the church. During the handover of services, there are more than 1000 people hanging around the platform outside the main hall. The scene is like the finishing of a movie show. The congregants finishing the service are busy registering for various church programs, making enquiries, chatting, or waiting for

\footnotetext{
${ }^{16}$ Since there are 3 entrances to the main building, multiple queues were practiced.
} 
someone else. Meanwhile, those joining the later service queue up and prepare for the service, and some are busy with kids and the child care service provided by EVC.

\subsubsection{The procedure in church is formal and systematic, if not hierarchical}

The format of service in EVC is rather formal and led by deacons or ministers, following the order of welcome, reading, hymns, prayer, sermon, offering, notice, as well as the blessing at the end of the service. On some special days, an orchestra will perform or missionaries from overseas may be invited to share. In general, the service is very structured and tightly scheduled. The interaction of the congregation in the service is kept to the minimum and people quietly pay full attention to the altar, the pulpit or the choir. People sleeping or chatting with each other is rare and people receiving phone calls may be advised, on the spot, against this.

Besides formal procedures in the service, participating in the EVC activities also involves a systematic procedure. An EVC member, in the anniversary publication, remembered her shocking and frustrating experience when she had to wait for few months before getting her kids settled in the child care service provided by the church.

To remind church members to pray, seven days of prayer matters are printed in the church bulletin: one day involves prayer for social issues, two days involve prayer for the global mission, and the other four days involve prayer for other church ministries or general matters, according to a theme.

\subsubsection{Cultural Values/beliefs}

In contrast to the other churches, the size of EVC is an outstanding feature that creates a unique EVC culture. The first of which is the mega church identity.

\subsubsection{Resources and size}

Being aware, if not proud, of their own size and resources is deeply rooted in the church and members' identity. The brilliant achievement of EVC can be clearly demonstrated in its development and expansion. According to the church document "EVC -- history and tradition" (2007), the development of the EVC can be divided into five major periods. The highlight in each period is the change in church location, church size, and the relevant increase in the numbers of church members. Starting in the 1950s, 
EVC was initially located in a 300 square foot area for only 70 people. Going through three more expansions across the decades, EVC moved to the existing location in 1990s, to accommodate more than 1,000 people in its 28,000 square foot main hall. Since then, EVC has continued to expand. To serve large numbers of congregations, EVC not only increased the number of Sunday services from one to five, but also purchased three more church buildings in the subsequent years. A church-supported social centre is housed in one of the buildings. As a result, EVC can now reach and serve more than 5000 members and even the local community.

The church expansion is not only important in the church history document, but also in people's minds. From the interviews in the present study, collective stories that can be recalled by members are, ironically, not many, except about the completion of the new church building. A EVC member notes:

Interviewee 1: what I remember doesn't mean the others will remember. .... Interviewer: Then can you talk about something you regard as important? Interviewee 1: the opening of the new church building.....because our church is really crowded, extremely crowded.....since the church expansion requires a lot of money, basically it is a huge amount of money, so more people talk about it.

The size of the church not only is about the number of people, but also the associated financial and religious resources, reflected in the comprehensive, if not diverse religious activities provided.

\subsubsection{Flagship religious and evangelical work}

Having said that the EVC provides diverse religious programs, their religious concern is still certainly orthodox, with an emphasis on religious and evangelical work. EVC is reputable with their decent and undebatable faith tradition tied closely to the bible. Their emphasis on the evangelical mission, whether locally or globally, also becomes their key foundation. A mission conference has been held annually in EVC since 1965, to educate the members about the importance of mission in the world, but the various kinds of short mission trips also give members direct experiences of what the global mission means to them. On top of that, the financial support of EVC to overseas missions is kept to one half to one fourth of their income. With its abundant resources and a respectable 
image, EVC gains credibility with Christian circles in Hong Kong. A member compares EVC to blue chips in the stock market:

Interviewee 3: Basically EVC is a sound church in good shape, because their faith is comprehensive. Their sermons .... are all rounded. They emphasise evangelical work. It is like if you want to buy shares, you would buy the blue chip stocks. EVC is the flagship of the denomination. To be honest, if you commit yourself to the fellowship, it is still possible (for you to find a role of yourself) even though it may be a bit difficult. But if you would like to have a cosy Christian life, EVC has first rate service. That means no one can bother you. You have your own work and family, and can be well fed spiritually. At some stage, after a rest and with good nurturing, people move again.

The church size and evangelical image is overtly shown in the built environment of EVC. The four church buildings, in 10 minutes walking distance, can make you feel like you inhabit a small community. In front of the newly founded EVC church building,

a decoration features grain, a picture of wheat, and a biblical verse: "lift up your eyes, and look on the fields, for they are already to harvest”. This is set up in the entrance of the ground floor. Above the decoration is a large plasma TV showing some gospel movies or sharing by Christian celebrities, so that passers-by can stay and watch. Following the escalator at the entrance, is a spacious lounge area where church members get together. In the lounge, besides a whole wall of fellowship notice boards, a display full of national characteristics is set up on the other side of the lounge. The countries on display include China, India, Arabic countries, as well as Laos, Indonesia, Thailand and Cambodia. In the annual mission conference, EVC will also hang up national flags in the main hall to raise the awareness of the global mission inherent in their tradition.

\subsubsection{Motivation}

EVC organizes diverse programs and, as an interviewee said, it is not a church with only one goal, and the emphasis on evangelical work reflects the urgency and obligation required of members to share God's love with others. The pastor in chief always reminds the congregations that they cannot allow God's love to stay in their own life without sharing with others. As an interviewee comments, members who are really "EVC" are also those who know God's love and love to share God's love with others. For 
example, one interviewee, who is the coordinator of a serving team, regularly visits an orphanage in the mainland China and organizes various kinds of service and moral education for the orphans. Another two interviewees work laboriously in the ministry preaching gospel to non-believers or nurturing new believers. As they understood, evangelical responsibility is "not just for some people with special talent and gift". Instead, as the gospels last long, sharing with others the good news is natural, if not obligatory. It is a style of living, and the true self reveals others when one has experienced God's love. Interviewees do not use the hard sell method to preach the gospel. Instead, they prefer to develop relations naturally and share with others through their life example.

Nevertheless, because of the huge church size and emphasis on formal and systematic procedure, the human touch and individual uniqueness in EVC is sometimes compromised. As a result, it is church growth, rather than individuality, that becomes the forefront of church ministry, as an EVC member observes:

Interview 3: EVC is so big that its main concern is about the system. In that way people are just like a product. I lead you to Jesus, sometimes like buying a product. Then we talk about the after service-nurturing them. Do you know my point? After they are nurtured and are ready to serve, they will get training. After the training, then you will be assigned to some other departments to serve. You are like a product in the church, brought back from somewhere, then being assigned, after service, to train up and then to serve.

\subsection{Summary of Cases}

In summary, the four religious settings have provided, for their members, very different contexts and environments in which they develop spirituality and well-being. The charismatic church (CHC) emphasizes encountering God explicitly and experientially, and develops an emotionally rich environment supported by praise and worship and other revival activities. Moreover, the expressive, spontaneous and enhancing environment and supportive interpersonal relationships together create very energetic and dynamic circumstances for people to pursue their faith. The social concern church (SCC), in contrast, emphasizes that religion is not only personal, but is also concerned with social, matters. SCC also realizes that faith is not only about words, but 
also deeds. As a result, it develops a very strong social concern and action-oriented religious traditions, sustained by various kinds of socio-political and consciousnessraising activities. Its critical understanding of faith, concern for social over personal matters, and the relatively distant, if not conflicting, interpersonal relationships, together give rise to challenging circumstances for people to seek faith. Like SCC, the goal of the fundamentalist church (FDC) is to realize the truth, whatever the cost and this is not easy to achieve. As with its mother church, FDC tries to emphasize the pre-eminence of truth

above everything and maintains various traditional practices (including breaking of bread, summer camp, and avoidance of religious symbols). The ambivalent attitude of members to rigidly following traditions can open up space for change. However, close ties and intimate interaction between members, supported by various kinds of religious and family activities, provide a spiritual family for members to understand faith.The evangelical church (EVC) also highly regards religious truth and the sharing of the gospel, and it is the flagship Christian church of Hong Kong, able to provide comprehensive religious education and evangelical programs that not many churches can offer. Nevertheless, because of its rapid expansion over the decades to more than 5000 members, EVC has to face the challenges, such as bureaucracy and organizational control, members lacking participation opportunities, and loss of a sense of community. In the next section, we will further discuss how the five key features are related to well-being in general. In the next chapter, we specifically focus on the key well-being variables measured in the survey section, and draw on interviewees' accounts and some psychological theories to explore how the various settings can influence or enhance well-being.

\subsection{Cross-case comparisons}

After presenting each case, the key features of the activity setting framework (Gallimore, et al., 1993) will be further discussed in relation to well-being in general. Activity settings data across cases will be summarized and highlighted again so that readers can comprehend the presence and absence of condition in each setting, which may help us further understand the relationship between religion and well-being. A more detailed explanation of its relation to specific variables in the survey - life satisfaction, social trust, civic participation, and sense of community - will be provided in the next chapter. 


\subsubsection{Personnel}

As discussed by O’Donnell et al. (1993) and Gallimore et al. (1993), people involved in an activity setting can tell us a lot about the dynamics within a setting and well-being. Whiting and her colleagues (Beatrice Blyth. Whiting, 1980; Beatrice Blyth Whiting, Whiting, \& Longabaugh, 1975) argue that the environment in which a child grows up, and the people the child got along with, have enormous impact on child behaviour. Maton (2008) also argue that the relational environment and leadership style of the setting can determine whether a setting is empowering or not, and whether the members feel empowered. The literature on sense of community (McMillan \& Chavis, 1986) indicates that the quality of people's interconnection and shared emotional connection within a setting is critical to the feelings of belonging and connection to each other, which is supposed to be a universal human need (Baumeister \& Leary, 1995). Literatures on social capital also remind us that the social network embraced by the people can have huge impact on their behaviour, interaction with others, or even the ability to attain certain goals. Organizational studies also highlight that human needs have to be fulfilled for the organization to survive (Scott, 2003). Symbolic interactionism also signifies the importance of generalized others in self definition. To understand the interplay between people and the setting and how the setting influences well-being, examining the relational aspect of people and the personnel dimension of settings is critical.

As demonstrated above, the four churches also have certain elements of empowering settings in terms of relational environment, opportunity role structure and leadership, according to Maton (2008). Nevertheless, the internal dynamics are different. The charismatic church (CHC) can be regarded as having a visionary and committed leadership, and a caring and supportive community with strong sense of community; the social concerned church (SCC) also have inspirational leader and shared leadership. However, because of conflict between church members, the relational dimension has been impeded at the social concern church. The fundamentalist church (FDC), like the charismatic church, has a shared and committed leadership, as well as a caring and supportive community with a strong sense of community. In contrast, the evangelical church (EVC) has nearly perfect role model leaders who are able to access resources, but 
the large number of people in the church and the high standards required of members who want to serve the church, hinder members’ opportunity to participate.

\subsubsection{Tasks}

The tasks and activities in which church members participate structure behaviour and experience. They govern the daily routine in which one may think, feel and act and also guide interaction engaged in with significant others. According to Cole (1996), it is in activity that people generate their psychology. Rogoff (2003) contend that it is participation in activity that people are transformed and developed. Furthermore, Giddens' (1984) notion of structuration, and Bourdieu's (1977) notion of habitus, highlight the importance of regular activities and routine social practice on behaviour and thought. It is not surprising to realize the beneficial effects of participation on well-being (Cantor \& Sanderson, 1999; Lyubomirsky, et al., 2005). Cantor and Sanderson argue that participation enhances well-being, by providing a sense of personal agency and purpose, a structure and meaning to daily life, and creating social connections. Lyubomirsky et al. argue how participating in intentional activities can combat adaptation , the hedonic treadmill, and sustain happiness. In the four churches, we find how closely the well-being is tied to the activities endorsed in each setting.

In summary, members of the four churches are provided with meaningful and engaging activities congruent to their cultural background (K. I. Maton, 2008). There are activities common to all settings, such as Sunday service, Sunday bible class, communion, prayer meetings and fellowships, and distinctive programs and activities in each church, which finally develop in the church members a unique character of their spirituality. While the charismatic church mainly provides activities to revive members' spirits in encountering God and community, the social concern church broadens members’ horizons and raises awareness and consciousness towards societal issues (Freire, 1972). In contrast, the fundamentalist and evangelical churches emphasize religious education and evangelical programs. The evangelical church provides their members with an extraordinarily comprehensive program to serve people with various needs, and the fundamentalist church emphasizes the breaking of bread in order to remember how Jesus died for people. In addition, family-type activities are also important. 


\subsubsection{Script}

As discussed by Gallimore et al. (1993), scripts are about implicit rules or patterns of interaction governing routine and everyday actions. The tacit rules tell us what is appropriate and govern what should be done or not done. Cole (1996) argues that script is an event schema that can specify action and can mediate cultural behaviour. DiMaggio (1997) adds that schema is a kind of automatic mechanism shaping perceptions and recalling information, unless people are particularly motivated to do otherwise. Goffman (1959) outlined ground rules of human communication and also highlights how rules facilitate interaction. Scripts not only provide ways for people to acquire the cultural practice of a community, but also the basic unit and building block constituting members' routine and daily behaviour from which well-being evolves.

The CHC values the lively, expressive and encouraging style of interaction and even accepts exotic behaviour, SCC appreciates concern for society over personal matters. While FDC pays no attention to liturgy, rejecting symbolism, the close interaction between members builds intimate bonding. EVC requires more structured, formal, and hierarchical interaction.

\subsubsection{Cultural Values/Beliefs}

Cultural values and beliefs reveal the heart of a community. They tell what is valued and important to the members. They are the shared symbol, concept and meanings systems within a community (Geertz, 1973; Carl. Ratner, 1999; Silberman, 2005). Although cultural values are supposed to be ideational, they are also reflected in the material world -- the activities organized in the settings, the accounts of members, as well as the built environment (Cole, 1996). The incarnated form of cultural value, like Vygotsky's (1978) notion of symbols and language, affect people's perceptions and thoughts, and even mediate human development. To understand development, including well-being, cultural values should be carefully examined. Some may argue for one unified culture, or fragmented culture (Martin \& Frost, 1996). This author believes that there are multiple dominant cultural values, along with underlying sub-cultural values. Nevertheless, in the present study, only dominant cultural values will be explored.

Cultural values of religious settings provide members a solid foundation for various kinds of emotional or behavioural development, enhancing different types of 
well-being. Each of the four churches demonstrates their own distinctive cultural ethos in which members develop faith. The charismatic church emphasizes experiencing God as a way to an abundant life; the social concern church advocates faith in action and openness. In contrast, the fundamentalist and evangelical churches highlight the supremacy of biblical truth and evangelical responsibility. It is observed that while fundamentalist church members show an ambivalent attitude towards faith from their mother church, a more accepting and caring culture, aligned with family type characteristics, evolves. The evangelical church, instead, allows more diversity because the size of population, even though religious and evangelical works are still primary.

\subsubsection{Motivation}

Purposes or motivation are not the only underlying forces driving people's action and behaviour, but also critical to well-being (Emmons, 1999; Karoly, 1999). Emmons (Emmons, 1986, 2005) explain that goals lend structure and order to people's life and also determine thoughts and emotions. As Emmons argues, goal attainment is the "benchmark for the experience of well-being” (2005, p. 733). In addition to the individual level, the importance of organizational goals on individual behaviour has long been recognized in organizational studies (Scott, 2003). In Cole’s (1996) understanding of culture, goal is also the key component. He explains how artefacts can mediate culture, highlighting that artefacts are not only materials, but also ideal because artefact is "the material world that has been modified over the history of its incorporation in goal directed human action” (p. 117). Oishi (2000) also suggests that the goal may be the moderator accounting for the difference of subjective well-being between cultures. Shinn and Yoshikawa (2008) note that being able to understand and measure the goal of a social setting is the key to changing the settings towards a more positive youth development.

To really understand well-being in relation to setting, understanding goals and motivation are important. Emmons (Emmons, 1999) even argue that religious and spiritual striving is one of three types of goal striving consistently related to well-being. In the present study, although the underlying motivation driving church members in various churches is more or less the same --to realize and witness their religious faith --to realize the goal of faith is quite different across the settings. Like cultural values, it is not unanimous for all people to share the same motivation; some hidden, if not dark, 
motivation was detected in the field study. In short, while charismatic church members strive to experience God so that they can witness God, social concern church members are taught to realize their faith by imitating Jesus’ service and even sacrificing for others. Fundamentalist church members try to express their fidelity to faith by sacrificing for truth, whatever the cost. They also acknowledged their limitation and God's love. Evangelical church members are encouraged to share God's love to others.

\subsubsection{Summary}

In summary, the activity setting has enormous impact on people's development, including well-being. It not only influences relational dimensions by its unique demographic composition and interpersonal relationships, but also helps shape the people’s frame of reference by cultural value, which is derived and sustained by the settings' underlying goal and motivation. Furthermore, activity setting embodies, or even materializes, its ideational dimension in the activity organized for people, the script guiding interactional behaviour, language used in the church or even in the architectural artefacts, with which people get in touch on a regular and a daily basis. Most importantly, the activity setting is not transient in nature, but institutionalised in regular practice on a daily basis, or over an extended period of time. It has lasting effects and the ability to transform the interpersonal processes into an intrapersonal one, and even make the internalization process complete, as Vygotsky (1978)and Bronfenbrenner (1995) argue. It is not difficult to imagine the enormous impact this kind of setting has on human development and well-being. However, activity setting is not completely deterministic. As Lewin (1951) and other psychologists (Bronfenbrenner, 1995; Riger, 2001; Rogoff, 1990, 2003) argue, it is the joint interactive effect (between human agents and the environment) that is critical to human development. In other words, given the enormous power of immediate settings on people, human agents still have a role. Sociologist DiMaggio (1997), after reviewing the research on the interplay between culture and human cognition, makes a useful insight. He notes that culturally available schemata constitute and inform our daily and automatic cognition, in perceiving or recalling information, and under the situation of schema failure, or when one pays special attention and is particularly motivated, people can "override programmed modes of thought to think critically and reflexively (p. 271)”. Therefore, DiMaggio is right to point out that 
culture can be constraining, but not overwhelmingly constraining so that there is no room for human agency.

After a brief summary of the similarities and differences of each setting and they relates to well-being in general, in the next chapter the author will apply the activity setting framework to further discuss how these differences of settings contribute to the significant findings shown in the survey section. 
Table 45: Synthesis of Activity Setting Key Dimensions

\begin{tabular}{|c|c|c|c|c|c|}
\hline \multicolumn{2}{|c|}{ Activity Setting Theme } & \multirow{2}{*}{$\begin{array}{l}\text { CHC } \\
\text { - Charismatic leadership } \\
\text { - Close membership } \\
\text { relation }\end{array}$} & \multirow{2}{*}{$\begin{array}{l}\text { SCC } \\
\text { - Inspirational leader } \\
\text { - Conflicts between } \\
\text { camps }\end{array}$} & \multirow[b]{2}{*}{$\begin{array}{l}\text { FDC } \\
\text { Equalitarian pastor } \\
\text { member relationship } \\
\text { - Close and sincere } \\
\text { relation between } \\
\text { members }\end{array}$} & \multirow[b]{2}{*}{$\begin{array}{l}\text { EVC } \\
\cdot \text { Humble and perfect role } \\
\text { model } \\
\text { - Members obedient and } \\
\text { loafing } \\
\text { - Lack of community }\end{array}$} \\
\hline $\mathrm{P}$ & $\begin{array}{l}\text { Personnel tell the } \\
\text { relational } \\
\text { environment of } \\
\text { the church and } \\
\text { the ideal model }\end{array}$ & & & & \\
\hline $\mathrm{T}$ & $\begin{array}{l}\text { Activity and tasks } \\
\text { structure the daily } \\
\text { routine of } \\
\text { people's behavior }\end{array}$ & $\begin{array}{l}\text { - Praise \& worship } \\
\text { - Cell group and other } \\
\text { revival activity }\end{array}$ & $\begin{array}{l}\text { - Social concern program } \\
\text { - Religious program not } \\
\text { strong \& personal }\end{array}$ & $\begin{array}{l}- \text { Breaking bread session } \\
\text { - Family type activity } \\
\text { - Evangelical activity }\end{array}$ & $\begin{array}{l}\text { Comprehensive } \\
\text { religious education } \\
\text { - Evangelical program }\end{array}$ \\
\hline$S$ & $\begin{array}{l}\text { Scripts: the tacit } \\
\text { rule and what is } \\
\text { appropriate in the } \\
\text { church }\end{array}$ & $\begin{array}{l}\text { - Free and expressive } \\
\text { style } \\
\text { - Exotic experience } \\
\text { - Enhancing language }\end{array}$ & $\begin{array}{l}\text { - Concern beyond oneself } \\
\text { - Not personal }\end{array}$ & $\begin{array}{l}\text { - Simple procedure } \\
\text { - Members' need were } \\
\text { concerned and attended } \\
\text { - Straightforward and } \\
\text { plain interaction }\end{array}$ & $\begin{array}{l}\text { - Long queues } \\
\text { - Systematic and formal } \\
\text { procedure }\end{array}$ \\
\hline \multirow[t]{2}{*}{$\mathrm{C}$} & $\begin{array}{l}\text { Cultural ethos tell } \\
\text { what is valued } \\
\text { and important to } \\
\text { shape the values } \\
\text { of members }\end{array}$ & $\begin{array}{l}\text { - Emphasis on the filling } \\
\text { by the Holy Spirit } \\
\text { - Abundantly rich life }\end{array}$ & $\begin{array}{l}\text { - Socially concerned and } \\
\text { action oriented } \\
\text { - Openness and diversity }\end{array}$ & $\begin{array}{l}\text { - Emphasis on the truth } \\
\text { over everything } \\
\text { - Ambivalence towards } \\
\text { the tradition } \\
\text { - Church as family }\end{array}$ & $\begin{array}{l}\cdot \text { - Resources and size } \\
\text { - Strong religious and } \\
\text { evangelical face }\end{array}$ \\
\hline & $\begin{array}{l}\text { Artefacts tell the } \\
\text { story of the } \\
\text { church }\end{array}$ & $\begin{array}{l}\text { - Fire/ harvest/ pigeon/ } \\
\text { colorful/ dancing }\end{array}$ & $\begin{array}{l}\text { - World Maps, couplet } \\
\text { - Station of Cross of } \\
\text { Jesus } \\
\text { - No air-conditioning }\end{array}$ & $\begin{array}{l}\text { - No symbol } \\
\text { - Not festival celebration }\end{array}$ & $\begin{array}{l}\text { - } 4 \text { church building } \\
\text { - Overseas culture display } \\
\text { - Harvest }\end{array}$ \\
\hline \multirow[t]{2}{*}{$\mathrm{M}$} & $\begin{array}{l}\text { The purpose and } \\
\text { motivation of } \\
\text { action tell the } \\
\text { church mission }\end{array}$ & $\begin{array}{l}\text { - To experience God } \\
\text { • To witness for Jesus }\end{array}$ & $\begin{array}{l}\text { - To follow Jesus' } \\
\text { sacrifice } \\
\text { • Faith in quest }\end{array}$ & $\begin{array}{l}\text { - To sacrifice for truth } \\
\text { - Any alternative way to } \\
\text { understand the faith }\end{array}$ & $\begin{array}{l}\text { - To share God’s love to } \\
\text { others } \\
\text { - Church growth }\end{array}$ \\
\hline & $\begin{array}{l}\text { Some hidden and } \\
\text { dark motivations }\end{array}$ & $\begin{array}{l}\text {-To pursue experience } \\
\text { for its own sake }\end{array}$ & •Human agency & $\begin{array}{l}\text { - Guilty feeling towards } \\
\text { God's love }\end{array}$ & - Production line \\
\hline
\end{tabular}

P- Personnel T-Tasks/ Activities C-Cultural value and belief S- Script/ routine M- Purpose of motive/ goal 


\section{Chapter 13}

\section{Findings and Discussion of the Qualitative Study}

\subsection{The effect of religion on well-being}

In this chapter, the findings provided by the activity setting analysis are discussed in relation to how various religious settings are able to influence and enhance well-being, specifically reflecting the variables under examination in the survey section. The religious setting is a complex system, as argued in Chapter Three, and the relationship between the parts is less constraining and deterministic. Instead of definitive answers, the author will try to provide an explanatory account for the significant findings shown in the survey section. Specifically, four key variables are the focus: sense of community, social trust, civic participation and life satisfaction.

\subsubsection{Sense of community}

As shown in the survey section, members in the four churches have a significantly stronger sense of community than their counterparts in the social centre. Nevertheless, there are also significant differences between the church settings. Members in the fundamentalist church (FDC) and the charismatic church (CHC) have a significantly stronger sense of community than those in the social concern (SCC) and evangelical church (EVC). We can understand this phenomenon by integrating both the sense of community literature and activity setting findings, especially the personnel and cultural value dimensions.

As proposed by McMillan and Chavis (1986), sense of community is defined by and heavily dependent on four aspects: the boundary of membership, the mutual influence between the member and the community, the integration and fulfilment of the needs of members, and shared emotional connection. These elements are identified in studied settings even though some of the dimensions may be interfered in specific settings. Firstly, in terms of the boundary of membership, although the four churches are open to non-believers, the majority of followers and common religious faith provide a clear boundary and ground for people to identify themselves. However, as mentioned previously, because of its location and 
good reputation in Hong Kong, it is believed that there are many passers-bys attending EVC. So, the boundary of EVC may be impeded.

Secondly, regarding the influence dimension, high levels of participation by members (except EVC) also creates a dynamic community in which mutual influences between members and the community are strong. For example, in the three churches (SCC, CHC and FDC), lay leaders (some are young adults) play a very active, even a leading, role in Sunday services or other ministries. In CHC, most of the worship leaders are young adults. In SCC and FDC, lay leaders can take charge of the sacrament. This was not seen in EVC. Moreover, church members in these three churches are also actively involved in, and take responsibility for, functional activities or church ministries. Members (especially in SCC and FDC) express that they are busy, if not burdened, with the church ministry, but they nevertheless enjoy and keep doing it. However, as McMillan and Chavis (1986) understand, influence is mutual and not a one way direction. For example, it was observed in SCC that young people (below 20 years old) can read the Bible in Sunday service. As explained by the deacon, it is one of their strategies to get young people to attend Sunday service. Through participation, members exert their influence on the congregation, but they are also influenced by it.

Comparatively, the size and culture of EVC seems to have a negative impact on participation, resulting in feelings of having less influence. The impediments of organizational size on participation has been documented in manning theory (Barker, 1968) and others' work (e.g. Wicker, McGrath, \& Armstrong, 1972). Wiken (1971) also gave notes several reasons for the negative effect of congregational size on participation, including difficulties in members' communication, few demands made on members because of the large pool of people, difficulties in monitoring members' participation, specialized roles, and the professionalization of some roles which may exclude members from participating. This accords with the high demands required of those who want to serve at EVC, and suggests opportunities for participation are also limited.

We came across this discontent often in EVC members' interviews. A young adult member remembers a time she was shocked by the rigorous requirements of merely attending a training course to serve, rather than actually serving. Another member also discussed the dilemma facing many church members:

Interviewee 3: In EVC, many people have said they want to contribute themselves but find no opportunity. This is true. While the church is big, it should be careful. Another 
reason is there are lots of people with ability. It is because we don't know about your faith if you are from the outside, we also cannot monitor you. However, it is different in smaller churches. The pastor will know tomorrow what you have said today. But in EVC, how long will it take to pass back to the pastor for what you have said? Therefore, it is right (for the pastor) to lead (the church) carefully. It is difficult to have it both ways. But you are right and I have heard many examples, saying that I am available, but.....

As a result of the limited participatory roles, in addition to the church size, EVC members find it difficult, if not impossible, to change the church. Nevertheless, it should be noted that the size of the church not only impedes members' participation in the church and their influence on it, but also hinders the church's influence on members. As previously mentioned, in 2007, about 2000 EVC members (about 40\%) were involved in serving in the church, but loafing is not uncommon. Two members lamented the "bigness" of the church and the inability of human strength:

Interviewee 1: Of course, the church is so big that sometimes you feel empty and pity...... Why? Many people came and go quickly. We cannot retain them, even with effort, because there are so many people. We have tried to do a lot to back up and make people familiar with each other, but it is very difficult for such a big church.

Interviewee 5: At the time I began to attend (EVC), there were about 1100 people; now this number has increased several times. At that time, the majority of people knew each other. Before you never saw people in the church take a bottle of water to drink, or heard the ring tone of a mobile, or people walking around or coming and going from the toilet. It would not happen before. To me, it is really disturbing. If the church is small, it is easier to control this kind of happening because everyone know each other and you also don't want to annoy others. So, it is easier. Let's say in the fellowship, it is easier to remind people not to be late. But you can't say too much when it is a huge church. Impossible! Do you introduce legislation? Do you actually confiscate mobiles? This is why I feel it is so difficult when the church is big.

For integration and fulfilling needs, committed leadership, close and caring relationships (especially in CHC and FDC), along with various kinds of meaningful activities, 
basically enable religious communities to become an empowering setting (K. I. Maton, 2008) in which the church members can learn and play, share and support each other. At EVC, although members find it more difficult to identify with the whole community, as some interviewees mention, the fellowship or smaller group is where they find their identity and sense of belonging. Furthermore, since many EVC members (or even passers-by) attend this church because of its reputation and comprehensive religious programs, they doubtlessly will not be dissatisfied with the resources and professional religious service provided in the church, sentiments unanimously expressed in interviews.

By contrast, because of its unusual religious tradition, SCC has to bear the cost which may restrain members' fulfilment and satisfaction. Externally, the socially-oriented religious faith of SCC may seem that it is considered marginal, or even heretical, in Hong Kong Christian circles. Internally, the open and critical cultural ethos makes SCC like an arena, rather than a comforting place. The concern about social, rather than personal matters, socially concerned activities, and less personal language, mean that members are less likely attend to each others' needs. As a result, this may affect the members' satisfaction within the community.

Fourthly, with reference to shared emotional connection, all four churches provide their members with a distinct but shared emotional connection in which they share their distinguished identity. For example, the exotic or ecstatic experience at CHC, the long Sunday service hours and family type activity in FDC, unique socially oriented religious action in SCC, noble Sunday service, and comprehensive Bible study in EVC. Nevertheless, it should be noted that there may be some restraints on developing a shared emotional connection in SCC, because of internal conflicts, and in EVC, because of its huge size. In SCC, because of conflict between camps in the church, church development is hampered, emotional pain and grievances have developed, and relationships are tense. Members could at least (as they claim that they occasionally can) get together harmoniously. This may also explain why three SCC interviewees mentioned their struggle as to whether to stay in church because of the culture and conflict in SCC, although they finally chose to stay. A member commented: "attending SCC, to a certain extent, is challenging and fluctuating."

In contrast, in EVC, it is not conflict that creates the problems, but the tiny feeling of the individual among the mass that makes it so difficult for people to develop the connection. The primacy of a huge bureaucracy or system and the secondary role of the individual, are 
often mentioned in the church document or members' accounts of EVC (such as church expansion and the production line metaphor). In the church anniversary publication, a senior EVC member looks forward when the collective memory in EVC can go beyond merely mentioning church buildings:

Today we always talk about collective memory. I don't know how to defend collective memory, but what I have is the individual memory........I not only use remembrance to defend my own individual memory, but also share my remembrance to call for others, finding their own individual memory in EVC as well. You need to have your own stories here, otherwise you do not belong to this place and this place does not belong to you. Our individual memory can weave into the EVC collective memory. Then the collective memory is not just about building,, but the abundance of God's grace. In summary, the activity setting findings, especially in terms of the relationship between members and participation (personnel) and distinctive cultural values, deepen our understanding of how religious settings enhance people's sense of community through McMillan and Chavis's suggested four dimensions: boundary, influence, integration of needs and shared emotional connection (1986). While the charismatic and the fundamentalist church are basically positive in all four aspects, it is also observed that the evangelical and social concern church seem to have some impediments related to certain dimensions because of church size and cultural ethos of their religious tradition. As a result, that may lessen religion's contribution to forming community. However our survey findings confirm that the evangelical and social concern churches still have significantly stronger senses of community than the social centre. We can say that the power of religion in SCC and EVC in the forming of community still exists, but is inhibited.

\subsubsection{Social Trust}

Regarding social trust, the survey findings show that religious groups have a higher level of social trust than the social centre, but only the fundamentalist church (FDC) members have a significantly higher level of social trust than those in the evangelical church (EVC) and social centre, while the charismatic church (CHC) and social concern church (SCC) members show no significant differences to those in the other settings.

In his review of the literature on trust and distrust in organizations, Kramer (1999) argues that, although some have conceptualized trust as a psychological state, others, either in 
sociology or psychology, regard trust as a relational concept. He also recognizes that much research on trust development indicates that trusting behaviours between people are "largely history-dependent processes” (p. 575). It is the interactional histories which help people calibrate and adjust the mutual trust expectation and subsequent behaviour.

The four churches provide a structure and institutionalized opportunity for people to develop interactional histories. The perennial activities provided by the churches are ideal places for people to calibrate their expectations and develop their trusting behaviour. In spite of potentially diverse opinions, shared religious faith gives people common goals and direction. This may explain why the members of four churches have generally higher levels of social trust than the non-religious group. Nevertheless, the significantly higher results of trust at FDC compared to EVC may be further explored.

From the FDC members' accounts, the close and sincere relationships between members and the family type activities seem to be related to people's mutual trust. Two members also reasoned that level of trust towards others is nurtured and developed within their immediate setting, and one of them also suggests that FDC's family type activity can facilitates mutual understanding between people in the church:

Interviewee 6: I think the trust you have towards each other is developed in your environment of growth, and depend on whether you have experienced or not the suffering or hurt. If you have experienced hurt before, you may develop suspicion. Maybe because our church is harmonious so we really can feel the trust between each other, at least we are sure the others will not harm you or cheat you......I think it is the life here, in which we gather together and participate in activities, that we know the community and each other. You will then find this group of people can be trusted and it is very natural in this kind of environment to develop trust. That may be the reason why those who regularly attend the church may have less suspicion towards the others.

Interviewee 5: I don't know whether it is related to the fact that our church has an activity called "member sharing session", which was set up in the early days by our spiritual leader. They are really good. Members sharing, means that it is not only limited to your fellowship. Normally after the service, we do not have many opportunities to communicate but in the "member sharing activity" every fellowship 
will be introduced to each other. We can share anything or business happened in our spiritual family -- the God's family. That probably enhances the cohesion.

As well as member sharing sessions, various kinds of family-type activities (e.g., family gathering function, church cleaning day, see p.196), mentioned in the activity setting analysis, and the many short, straightforward, interaction opportunities (e.g. interaction in breaks, chair arranging, p.198), all create opportunities for member to meet and interact. As members realize, every member have at least some basic understanding of each other in the church. All these enhance mutual trust between the members.

In addition, from members' accounts, it is apparent that the pre-eminence of religion has certain effect on mutual trust. As a member mentions on p.195, members' sincere relationships are not only shown in support and positive feedback by brothers and sisters, but also in reminders and even blaming others for wrong-doing, according to religious truth. Therefore, to FDC members, the challenge is not the speaking or not of truth, but how to "speak the truth in love", as a member noted. We can believe the relatively clear cut religious belief in FDC provides its members with a sacred foundation and trust. Moreover, although it has been argued that FDC members have shown ambivalence towards their traditional holding of religious ideal, Pratt and Doucet's (2000) examination of ambivalent feelings also sheds some light on understanding FDC members' close bonding, which may facilitate trusting behaviour. They argue that, when there are ambivalent feelings towards the organization, people have four types of responses, depending on the attitude (either positive or negative) and movement (either approaching or avoiding) they adopt. They argue that those who adopt a positive/approach response will get closer and move towards others to feel safe to resolve ambivalent feeling. From FDC member interviews, it is also observed that although they sometimes mention falling behind in their religious ideals, and the subsequent ambivalent feeling toward those ideals, they often note that their church is their family in which they lay their heart. By moving towards others they resolve both their love and hesitation in those religious ideals.

We can see the impact of activity setting on the development of social trust in FDC members. Synergy is created by sincere relations (personnel), family type activities (tasks), straightforward, direct, plain and open interaction (scripts), and emphasis on truth above everything and associated ambivalent feeling (cultural belief). Together this creates a true, 
genuine and truthful relationship between members, a foundation to build trust. Nevertheless, interpersonal trust within the immediate setting has effect beyond the immediate circle. In her article "Culture and social behaviour", Whiting (1980) argues that the habits of interpersonal behaviour that is learned in settings can be generalized to other settings. A truly and reciprocal trusting relationship developed in FDC not only facilitates stronger interpersonal trust between members, but also later transfers to people outside their immediate setting.

Following this line of argument, we can understand the relatively lower levels of social trust among EVC members. Since it is personalized knowledge about one another which provides the foundation of trust (Kramer, 1999, p. 576), the size of the setting and the opportunities provided for repeated interaction may also affect the development of this kind of personalized trust. Apparently, the size of EVC constrains opportunities provided for repeated interaction to develop personalized understanding, let alone mutual trust. For example, a senior member who attended EVC for more than 20 years witnessed the change in church size and how this change affects interpersonal relationships between members. In the past, many EVC members attended Sunday bible class regularly throughout the year. Participants could develop deeper relationships and understanding of each other. Nevertheless, she observed, as the church expanded, the flow of church members became so frequent that relationships between members (or between students and teachers) could not be built. She comments: "because of excessively high mobility, all are short-lived.” Formal, if not distant, interaction is now the norm. These formal interactions, in addition to normative obedient attitudes towards leadership by members, further hinder authentic and straightforward interpersonal relationships between members. For example, a member observed that agreements in EVC are often reached only on the surface, rather than wholeheartedly. Some believers, who may disagree with the pastor, normally keep silent out of respect for authority and unity.

The activity setting findings are helpful to make sense of the operation and mechanism of social trust in the evangelical church. The size of the church, obedience towards the leader (cultural belief and personnel), and the proper, formal and official ways of interaction between people (scripts), together inhibit more genuine trustful relationships between people in the immediate setting, which subsequently limits the salutary effect of religion on social trust. 


\subsubsection{Civic Participation}

Regarding civic participation, the survey findings show that only social concern church (SCC) members showed significantly stronger levels of social participation than those in other settings, be they religious or non-religious. The other settings do not show any significant difference from each other.

To understand religiously based civic and political activity, political theorists Wald et al. (2005) note three dimensions: the motives drawing religious groups into action, the means and resources that enable religious groups to sustain participation, and the opportunities available for religious groups to enter into the political realm. To understand the question as to how and why SCC is able to enhance civic participation, we can focus on examining the cultural dynamic and resources available in SCC to reveal the motivations behind members' civic behaviour, as suggested by Wald et al. (2005) and Wood (1999).

As documented in activity setting analysis, SCC members view religious faith as not only an individual matter, but as going beyond the self, to a broader, collective level. Their socially concerned and action-oriented interpretation of religious faith, with the various symbols and associated images (such as the heroine story or built in artefacts), helps frame and build members' shared and unique identity, as reflected by a member:

Interviewee 4: They [ SCC members] feel that participating in society is what believers have to do. It is their mission, because Jesus lived a life, not just within the church and reading the bible, praying or meditating, but also reaching out to people. This provides us with great inspiration to take part in human life. Taking part means to realizing our faith through serving others. I volunteered in a home for the elderly a few years ago. I go to the home for the elderly to dance and perform sometimes. We think it is very common, not something special.

To participate in democratic life, researchers highlight the importance of the psychological underpinnings of political tolerance (J. L. Sullivan \& Transue, 1999), or capacity for ambiguity (Wood, 1999). Political tolerance, evolving from the concept of religious tolerance, means living with ideological and political enemies (J. L. Sullivan \& Transue, 1999). Wood (1999), drawing on the Weick's (1979) ideas, argues that for organizations to participate in the public or political realms they have to develop an interpretive lens that should be flexible and nuanced enough to understand a complex 
political world. As such, SCC's cultural values of openness and diversity, even towards religious faith, make it an ideal for members to substantially participate in the civic world.

On an ideological level, SCC provides rich cultural resources for people to draw on to participate in civic life, and the liberal theological faith also helps members not to just confine their horizon to religious faith in the immediate setting, but to engage actively in broader society and to interact with outsiders.

Civic participation is not only justified on the ideological level, but is upheld on the relational and material world. In SCC, the relatively distant relationships between members creates weak ties (Granovetter, 1973), ideal to develop bridging rather than bonding social capital (Putnam, 2000; Wuthnow, 1999). The routine activities and the daily scripts in the settings also help maintain and sustain this kind of behaviour. As explicated in the activity setting analysis, SCC provides member a wide range of action and socially oriented activities. This raises the awareness of the societal issues, and also provides participation opportunities. Moreover, the daily script of SCC often activates the ideal self beyond one's immediate interest. We can find this kind of association in a member's account:

Interviewee 2: SCC has a tradition - to serve. The pastor, at the end of service, will ask you to serve people. I really feel that not many churches say this - to serve. To be honest, sermons at other churches are more appealing than that in SCC, which are boring. However, I feel that who I am is nurtured. Why I am different from others is because someone in SCC has implanted something in me when I was young.

Interviewee 2: SCC has really given me a sense of social concern, from a young age. I still remember how I helped to deliver meals when I was at primary school. We did that every year at that time. We had been driven out even by laundry pole. We learned to know the different people in the community, mainly the elderly, single parents and those living alone, and learnt how to take care of them. That is the great lesson I learned at SCC. There have been criticism of SCC for its openness towards many issues, including its discussion oft AIDS or homosexuality. This is totally different from the other churches where these kinds of issues cannot be discussed.

We have observed the higher levels of social trust in the fundamentalist church (FDC). We also note that the social concern church (SCC) provides a unique cultural environment 
that maintains and sustains interest and behaviour in relation to the civic life, leading to higher levels of civic participation. That means, although there are weak ties between members (personnel), the socially concern program (activities), the language which is less personal but socially oriented (script), the cultural belief that is grounded in concern beyond oneself, and the open, if not critical, reflection on religious faith, all contribute to a strong civic sense institutionalized and implanted in members’ minds and their life.

Using this perspective, we can easily understand why other religious setting cannot facilitate members’ civic participation (especially CHC and FDC). Firstly, in relational dimension, intimate relationships between the members within the settings help build strong bonding social capital, but hinder development of bridging social capital (Putnam, 2000; Wuthnow, 1999). Secondly, ideologically, primary concern is towards internal affairs rather than societal matters (experiencing God in CHC and family issues in FDC). Members are not prepared on the ideational level to engage in social events or face societal challenges. Furthermore, the relatively clear-cut, if not rigid, fundamentalist religious worldviews -emphasizing the absolute primacy of religion above everything -- also ill-prepares members to be politically tolerant and open and participate competently in civic or political worlds. Certainly, their mainly religious-orientation and associated activities do not provide them with enough cultural resources and tools to articulate the complex political world and mobilize members towards social issues (Wood, 1999). Although EVC does organize some social concern program and does not totally ignore the existing world (the pastor also encourages members to vote on election day), the focus is primarily focused on moral issues and the programs are relatively sporadic, if not ad hoc, compared to frequent evangelical meetings and activities. Thus, the impact is less powerful.

Again, activity setting analysis helps us understand how different religious setting can promote or constrain well-being, through ideational (cultural values), material (activity and scripts), and relational (personnel) aspects.

\subsubsection{Life satisfaction}

In terms of life satisfaction, survey findings demonstrate that members in four churches (CHC, SCC, FDC and EVC) also have stronger results than their counterparts in the social centre, even though only the charismatic church (CHC), the social concern church (SCC) and the evangelical church (EVC) attain statistically significant results. The focus here 
is to examine the not outstanding result shown in fundamentalist church (FDC), and the possible different mechanisms underlying the other three settings having significant results

As documented in the literature (see Chapter Two), relationships are shown to have a significant impact on subjective well-being, and the sense of belonging is also a universal human need (Baumeister \& Leary, 1995). The beneficial effects of sense of community on subjective well-being have been documented (Davidson \& Cotter, 1991). At first sight the relatively stronger sense of community in the four churches (shown in survey) seems to help explain why the members have higher levels of life satisfaction than non-religious groups. However, logically then, we cannot easily understand why FDC has a relatively lower level of life satisfaction when they have a relatively stronger sense of community.

As mentioned in the literature review, pursuing goals was also shown to have a large impact on life satisfaction. As Emmons(1986) and King (2008) argue, simply having a valued goal to strive for is related to subjective well-being. The beneficial effects of pursuing goals can be further enhanced if the goal is personally valuable, instrumental to human needs, moderately challenging, and compatible with the endorsed cultural value (Diener, et al., 1999; Emmons, 1986; L. A. King, 2008). Emmons (1999) noted that spiritual striving is one of the three personal strivings often associated with well-being. In the current study, it was also found that members in each church internalized the immediate setting's cultural goals as their personal goals, which brings out the diverse manifestation of beliefs, experience and behaviours, finally leading to different levels of subjective well-being. For church members, having a community to identify with, as well as having a spiritual goal to strive for, endorsed by and aligned with cultural value in the immediate setting, enables members to have a generally higher level of life satisfaction than the non-religious comparison group. However, differential results between the churches are worth further examination: the CHC and FDC churches have contrasting characteristics. Then, to explain the life satisfaction of members in EVC and SCC, the author proposes different mechanisms are in play. Speaking overall, it is the goal pursued in different churches that matters and creates the different levels of life satisfaction.

According to King (L. A. King, 2008) and Fredrickson (2008), one of the promising ways to increase subjective well-being (including life satisfaction) is to enhance people's positive affect. King argues that, not only can participating in activities which enhance positive affect help in the short term, but also living a rich emotional life, incorporating both 
happiness and occasional unhappiness, is effective in bringing long term change to subjective well-being.

In our activity setting analysis, we can note that CHC provides an emotionally rich environment, reinforced by lively, expressive praise and worship (activities), accompanied by enhancing language and exotic and charismatic experience (scripts). Most importantly, this kind of practice is driven by a cultural belief that believers are privileged to encounter God and the goal is to experience God face to face. As noted by a CHC member, the emphasis on inner feelings and encountering God in CHC enriches members' emotional lives:

Interviewee 1:The evangelical church may also have religious experience, but in traditional church I feel that the experience is not to the soul. You seldom hear that Christians can convert and confess to God. I did not hear these things in my previous church, but it is emphasized in the charismatic church. The feeling is stronger and there are more emotional experiences, inner healing, and confessional experience... I feel that I am more able to feel, be more human, and have more emotions.

Fredrickson (Fredrickson, 2000, 2001, 2005) notes that these positive emotions, such as joy, interest, contentment, or gratitude benefit emotional well-being. As she argues, positive emotions not only broaden members' thought-action repertoires, but also build personal resources, such as physical resources, social bonds or intellectual creativity, which have lasting effects. From the interviews, it was found that speaking in tongues by CHC members not only enable them to express feeling, but also uncovers hidden emotions or "reality" and this expands their thoughts-action repertoires.

Interviewer: Do you speak in tongues naturally?

Interviewee 3: Yes. When you use this way to pray - speaking in tongues, and crying out your feeling to God, you know, some pain cannot be expressed by language, but after speaking in tongues, you can feel great satisfaction in your soul......

Interviewee 1: Recently I have an experience. When I pray, I pray intellectually ${ }^{17}$ in the beginning, but later in my prayer I suddenly have a feeling that the relationship between my sister in law and her husband is not very good. Then I asked my wife to see if she knows about that. My wife says she doesn't know and she doesn't think

\footnotetext{
${ }^{17}$ Intellectual prayer, according to interviewee 2, is prayer using language understood by humans, using thought and reason.
} 
so.... Then I continue pray for them (in tongues) and feel that the problem is becoming very serious. Later I find that they really have a problem in their marriage and have separated already. If you ask me about my prayer, I may not know about it in detail, but the direction is to pray for their marriage. When I prayed, I became very emotional but didn't know why. I also didn't know what I prayed for. Then I prayed until I was exhausted but still didn't know why. But lastly I know the answer. As well as broadening thought, it can also be noted that the exotic experience of CHC members after they encounter God often create opportunities for people to communicate and exchange, which strengthen social bonds or intellectual creativity. A member recalled another member's exotic experience - speaking in tongues - and how this built a special relationship. Interviewee 2: There is a brother who went on a short mission trip.....(probably to France).He did not how to speak French, so he prayed in tongues for another brother... Normally nothing happens on the spot..... Then he flew back to Hong Kong...... After a period of time (one or two years), that overseas brother came to Hong Kong to find this brother. He had to find this brother because from translation he said, "I have done what you asked me to do!" The brother in Hong Kong felt puzzled and asked what he had asked him to do. The brother replied that "you asked me to build a church, then now what should I do next?" This is speaking in tongues. Furthermore, Fredrickson (2001) contends that positive emotions "undo lingering negative emotion” (p.221) and "fuel psychological resiliency” (p.222). In our data, this can be effectively demonstrated by an interviewee who draws on her spirituality and religious community resources to face her many years of struggle with her nearly broken and violent relationship with her husband.

Interviewee 3: I have tried many ways to save our relationship by myself, but did not succeed. I could not do anything, but felt fatigue and powerlessness. The only thing I could do was go to church, pray and cry for His giving me a heart with strength........ When I reflect about the relationship with my husband in these few years, it has been very difficult indeed. But every time (in the service), I am also the first one to stand up and go near the altar. Then I can cry in front of the Lord or be blessed by others. Even if I do not cry, or nobody prays for me or gives me a blessing, it is still a kind of healing only if I can cry before Him (God) or speak in tongues before Him. The reason I can endure so many years is through God's healing. 
Emotional exhaustion, stress and burning out occur, but the negative side effects can be mitigated by carefully designing the organizational environment, such as providing social support and opportunities for the people to release their emotion, which can have supportive and cathartic effects (Adelmann, 1995; Hochschild, 1983). From the interviewee's comment just quoted, the supportive and accepting environment of $\mathrm{CHC}$ for even unusual expression release the members' emotion, resulting in beneficial effects. A CHC pastor also sought to understand members' high levels of life satisfaction:

Pastor: At the beginning we also thought that they might have an easy life, but it was not true. Over the last few years, particularly the last year, we have organized camps called "encounter weekends". We separate male and female members so we can touch something deeper. We discover some of sisters face many problems that we cannot touch or handle in cell groups... Some inner problems: issues related to development, childhood, parents, and family. We even found that some members had been sexually abused. All these issues could not be discussed in cell groups or disclosed in Sunday services. However, the camp provided an alternative space for those issues to be addressed, through spiritual healing, prayer or counselling... To women, these kinds of services are very important. In those two days at camp, they can cry, comfort each other freely.... These programs help sisters address their inner issues, thereby enhancing their wholeness and integrity.

That is not to say that other churches do not have this kind of positive emotion; however, strong and intensified emotion can be expressed in CHC and there exists an emotionally rich environment, sustained by relevant activities (revival meetings), interactional patterns (speaking in tongue), and cultural norms and beliefs (emphasis on experiencing God vividly and explicitly), which have far-reaching and impressive effects.

Conversely, the ambivalent or guilty feelings shown at FDC seem to have inhibitory effects on life satisfaction. A member remembered there were several times when the preacher asked members whether they felt satisfied with life, and just a few people responded while most members were silent. In the activity setting analysis, it was also observed that the breaking of bread session is highly regarded to remind the members of Jesus's love for humankind and their sinful nature, as not deserving God's love. In addition, FDC members were always reminded of the biblical truth above every human desire and earthly comfort. Even if FDC members do not fully embrace those religious ideals, the feeling of falling 
behind in their religious idealism, and the associated guilty feelings, has been clearly mentioned in interviewees’ accounts. Kennedy (1999) and Murray and Ciarrocchi (2007) argue that spiritual struggle and guilty feelings can suppress positive relationship between religious faith and subjective well-being.

However, King (2008) argues that occasional unhappiness and negative experiences are necessary for people to have a rich emotional life and for subjective well-being, to avoid the hedonic treadmill. Rubin (2008) also notes that religious melancholy is a commonly associated with evangelical pietism. As Rubin argues, the spiritual itinerary of evangelical pietism is sin to salvation, along which people have to "experience an initial Godly sorrow for sin", then "lose himself or herself in despair and thus seek redemption and healing as a child of God” (p.294). As Rubin understands it, only through the terrors of conscience, despair and hopelessness, can the lost sheep return to God and renew their mind and heart through grace. To evangelical pietists, melancholy is an important sign, if not a necessary step, in a spiritual journey.

Having said that, Rubin also recognizes that this melancholic type of devotional piety has its limitations, such as weakness of faith, excessive meditation, scrupulous conscience, or even doubt over redemption. King (2008), despite acknowledging the importance of negative experience to subjective well-being, also admits that a persistent low level of subjective wellbeing may indicate to people "the need for life changes, goal re-evaluation and a reconsideration of life meaning” (p.443). The unique kind of religious piety in FDC may induce in members an experience of falling behind in their religious idealism, and the associated guilty feelings, particularly for those who may not be fully embrace the religious ideal of the setting. It is not only institutionalized mentally (as a cultural value), but also materially (activity), finally inhibiting members’ life satisfaction. This kind of inhibitory effect was discussed by an FDC interviewee who courageously, painstakingly, and successfully strove to separate from her gambling-addict husband. Originally, she wanted to share, with many others, the amazing story and the providence God had given to her in the process; however because of the traditional and conservative attitude towards divorce in the church, and because of other church-mates' advice, the woman kept silent and just shared with a small circle of brothers and sisters within the church. Therefore, despite close and caring relationships and a strong sense of community existing in FDC, and their associated 
positive effects (K. I. Maton, 2008), the enhancing effect of religion is impeded because of unique religious beliefs and practices associated with the settings.

Compared to CHC and FDC, the author argues that the pathway to life satisfaction in EVC and SCC may be different. Instead of having a strong sense of community and of an emotion-promoting environment like CHC, SCC and EVC may provide other means by which people to attain subjective well-being. In the author's opinion, this may be related to pro-social behaviour nurtured in SCC, and the resources, richly provided in EVC, which help the members to meet religious needs.

In our study of SCC, it was observed that living a life of service and concern for others is not only encouraged, but also leads to greater life satisfaction, even though it may sometimes bring them inconvenience or even trouble. For example, a SCC member, after helping a student with a disability through his work, experienced a deep sense of satisfaction:

I: Religious faith helps us to be concerned with those around us, especially when we understand the life of Jesus and his teaching, which gives us inspiration..... Believing in Jesus's love for us, when we see others' needs and then respond with care and contribute ourselves, and the joy we gain is huge because we can see changes in others. The support, comfort and satisfaction we can draw is quite large.

In the activity setting analysis, we also find other examples of how SCC members persist in their altruistic behaviour or service to the community, even if facing challenge or rejection, but still feeling proud of their persistence. Collectively, despite being criticised for the great an emphasis on service and social matters, as opposed to religious matters, SCC still identifies strongly with this social and other-focused orientation.

The benefit of altruistic or pro-social behaviour to self-esteem has been recognized (Batson, 1998). Fredrickson (2008) argues that doing good and helping others can generate positive feelings, thereby enhancing subjective well-being. Thoits and Hewitt (2001) also observe volunteer work is closely associated with six aspects of well-being: happiness, life satisfaction, self-esteem, sense of control over life, physical health, and countering depression.

Although the classic literature on altruism assumes that altruistic actions are mainly due to egotistical motives, Batson and his colleagues (Batson, 1998; Batson, Ahmad, \& Lishner, 2009) argue against egoism as the only motivation underlying altruism. Instead, drawing on over 30 experiments, they tentatively conclude that "feeling empathy for a person in need does indeed evoke altruistic motivation to see that need relieved” (Batson, Ahmad, \& 
Lishner, 2009, p. 424). They further propose two other forms of pro-social motivation: collectivism and principalism. Collectivism as a motivation is about benefits to the collective and whole group; principalism as a motivation is about higher moral principles. Our findings related to SCC seem to suggest that pro-social behaviour in SCC members is closely related to religious ideals, which accords with the principalist explanation.

The open attitude of SCC also helps members handle contradictory life events (such as the divorce of the pastor, and concern for AIDS related issues). A member also remembers how the liberal religious tradition of SCC helped her face the reality that Christianity has been detrimental in some situations (like in South Africa). Moreover, the liberal SCC tradition helps people to get along across different cultural backgrounds. Fredrickson (2008) also notes that the limited existing research tends to support the link between openness and positive benefits. This seems to suggest that SCC's socially concerned orientation, as well as its openness, are able to enhance people’s positive affect, which further strengthens life satisfaction.

In contrast, it was observed that EVC members have a sense of contentment when participating in church activities or serving the church ministry because, in the process, they experience the presence of God, which is satisfying.

Interviewee 4: The presence of God is really important. The feeling that... if you have experienced God before, you will know it is truly......truly......it is difficult to explain the pleasure or say, the feeling being with God is like nothing the world can offer.. As Cantor and Sanderson (1999) argue, participating in personally and culturally valued activities, permitted by available resources and opportunities, can enhance well-being. In addition, as Pargament and his colleagues (Pargament, 2002b; Pargament, Magyar-Russell, et al., 2005) argue consistently, religion is a unique source for well-being. Due to abundant resources, EVC is able to provide a wide range of programs and services, addressing people with various religious needs. It has been argued before (13.1.1) that the participation opportunities for members in EVC are limited, but there are still a wide variety of activities to join and attend, through which people can encounter the divine. It is not difficult to find EVC members feeling satisfied with the marvellous and professional service provided in the settings. EVC not only provides members with many activities, but also, through the participation, members are able to experience spiritual renewal. As a member commented, EVC is a "magnificent temple for people to enjoy the resources inside, even though they may 
not feel they belong to this church.” This interviewee also adds that EVC is also an ideal resting place for those who are burnt out to recover spiritually, after serving in the other churches, or for those who were overwhelmed by busy, modern society. Nevertheless, the author is not saying that other churches cannot facilitate life satisfaction through a spiritual route. In reality, all the interviewees in the study highlighted the important role of religion in their lives, whether in the meaning making process, or by having a rich emotional life, or living a life concerned with others. However, more EVC members, relative to other settings, mentioned their satisfaction in drawing on religious service provided by settings.

In summary, while strong and positive emotions may be one of the drivers leading to life satisfaction in CHC, it is believed that EVC and SCC offer other pathways to enhance satisfaction. Instead of nurturing members to be more emotionally sensitive so that they can draw on positive emotion to gain life satisfaction (as with CHC does), SCC, instead induces in members a strong belief in self-sacrifice and serving others, so that they can derive satisfaction from their involvement in those acts. By contrast, EVC, by emphasising religious development and using abundant resources, enables members to experience God solidly.

To understand the cultural influence on well-being, different pathways to cultural difference on SWB have been documented. Suh (2000) suggests the self as the hyphen between culture and subjective well-being, and Oishi (2000) recognizes that different goals embraced by people in different cultures have a moderating effect in their subjective wellbeing. Markus and Kitayama (1994) argue that the involvement, or not, in culturally appropriate behaviour determine whether people can feel good about themselves and satisfied with life. The current findings seem to suggest that various kinds of religious environments can enhance satisfaction in multiple ways. The present study seems to support Oishi (2000) who argues that different goals embraced by people (in different cultures and different immediate settings), can have a moderating effect on subjective well-being.

\subsection{Summary}

The activity setting data help to demonstrate how different religious settings provide church members with a unique cultural environment facilitating their individual development. The activity setting provides people with a goal towards which behaviour and action is directed. Nevertheless, striving towards this goal creates varieties of cultural values, activities, scripts, and interpersonal relationships. While the cultural values provide people with a 
shared belief and common understanding on the ideational level, tasks and scripts are concerned with the tangible world and govern behaviour on a daily basis. Bourdieu (1986) discusses the notion of capital, important here; the religious community provides rich religious and socio-cultural resources on which members can draw to enhance well-being. The motivation dimension of the setting provides people with a personal goal to strive for; the personnel aspect of the setting provides people with a social network of resources; activities organize people's daily routine; scripts implicitly guide interaction. Moreover, the cultural ethos not only maintains and sustains, but also makes sense of on-going practices. All these finally lead to individual development. The effect of the setting can be expressed in multiple facets: the members’ relational side, participatory opportunities, behaviour, interactional patterns, belief and motivation, as well as emotions. It is believed that the synergy and lasting effect created by this unique setting enhances the well-being of people in different religious settings in different ways.

The previous section demonstrated that the charismatic church provides members an emotionally rich environment so that emotion be released, thereby achieving catharsis, but also positive emotion can be induced, broadening thought and building personal resources (Fredrickson, 2001, 2005) and a higher level of life satisfaction is attained. In contrast, the social concern church's action and socially oriented faith (passed on from the previous generation and sustained throughout the activity setting) help frame and shape members’ understanding, interpretation and execution of faith, leading to high levels of civic participation as well as life satisfaction. The fundamentalist church, with its nurturing environment and sincere interpersonal relationships, ties members together in a process that build trust towards church members and even social trust towards outsiders. The evangelical church, with its huge size, seems to inhibit the building of social trust and a sense of community towards the community, even though it is able to enhance satisfaction with life. It can be concluded that different religious settings enhance different kinds of well-being, by different ways of influencing emotions, cognition or interactional patterns.

Nevertheless, the salutary influence of religious settings is not only through enhancing different kinds of well-being, but also through providing different pathways to the same kind of well-being. In our study, although charismatic church members' level of life satisfaction is the highest, social concern and evangelical church members also demonstrate higher and significant findings than the non-religious group. Our qualitative and interview data suggests 
that the social concern church members acquire life satisfaction through embracing the religious belief of self sacrifice and through serving others. In contrast, the evangelical church members' find life satisfaction through abundant resources supporting their search of faith. Social concern and evangelical church members' route to life satisfaction is different from that provided by the charismatic church with its emotionally rich environment that encourages positive emotions.

In summary, religious settings provide members with rich social and cultural resources to develop well-being, and enhancement of well-being is manifested in diverse ways. Firstly, settings may provide for members unique cultural environments where they can develop distinctively emotional, cognitive and behavioural patterns, leading to different types of well-being. Secondly, the culture created by different settings may pave different pathways leading to the same kind of well-being. One may be the emotional pathway, others are the thinking pathway and the behaviour pathways. Based on both our study and the literature, we can argue that the cultural environment has enormous influence on well-being. In addition, the ways culture leads to well-being is intricate and diverse. 


\section{Chapter 14}

\section{Overall Discussion}

Mental health has long been associated with the absence of mental illness, rather than the positive notion of well-being. It is only in recent decades that well-being has received the attention of empirical researchers, through the positive psychology movement and concern about prevention. Spirituality and religion, despite being part of traditional wisdom and human resources, has only recently garnered greater attention from social scientists concerning its importance to people's lives, including well-being. The aim of the present thesis is to further the discussion of the topic and to examine whether, how and why Protestant spirituality and religious community can enhance well-being in an Asian city -Hong Kong. Although Protestant Christianity is practised by only about 5\% of the Hong Kong population, its impact and influence on the development of Hong Kong society has been huge, especially under British colonial government. In this final chapter, the overall findings of the study will be summarized, and their implications for the psychology discipline and the scientific community will be discussed. Last, but not least, the strengths and limitations of the present study and the direction for future research will also be examined.

\subsection{Overall findings}

The researcher recognizes that both spirituality and well-being are multi-dimensional concepts and their relationship is complex and not direct. The MANOVA findings demonstrate that the positive influence of religious context on people's spirituality and sense of community is medium to high, but the positive influence on well-being is small, but still significant. Nevertheless, the effect of religion on well-being is not simple and directly correlated, but largely depends on the context and the kind of well-being. To put it simply, in this study, members of the three churches were shown to have higher level of life satisfaction compared to the social centre. These were the charismatic church, social concern church, and the evangelical church. In terms of social trust, higher levels of social trust were seen in the fundamentalist church compared to the social centre. Regharding civic participation, members of the social concern church have significantly higher results than the social centre. 
Discriminant analysis finding further consolidates our MANOVA findings and demonstrates that different settings are characterized by different mixtures of variables and patterns of behaviour. Not surprisingly, the non-religious group is characterized by lower levels of spirituality. The socially-oriented church is singled out from the other stricter type religious settings, reflecting their unique kind of spirituality with an activist outlook. The evangelical church is characterized by a weaker sense of community and high social flexibility, reflecting the potential effect of church size. Last, but not least, the charismatic church is characterized by a higher level of life satisfaction and reward for application, while the fundamentalist church has a higher levels of social trust, but also social cynicism.

Therefore, it can be said that people in religious settings are more spiritual (in terms of spirituality-related variables used in the present study), but the effect of religion on their well-being is not linear and simple. If a person is more religious, it does not necessarily follow that they have a higher level of well-being. Instead, the manifestation of well-being is closely related to religious context.

As well as the influence of religious settings, regression and path analysis findings further explored the relationship between spirituality and well-being outcomes at the individual level. Findings demonstrate that spirituality has both direct and indirect effects on well-being outcomes, either directly influencing the outcome or having its influence mediated through the other variables. Specifically, the influence of spirituality on personal (life satisfaction) and interpersonal well-being (social trust) is fully mediated through mental health and a sense of community. Spirituality can directly predict civic participation, even though its influence is also partially mediated through voluntary organization membership.

In other words, spirituality influences well-being through both unique and mediated pathways, depending on the kind of well-being variable. Nevertheless, the path analyses also clearly show the close association between spirituality and the sense of community, as well as the importance of this contextual influence on well-being. That means that spirituality not only affects well-being directly, but also can predict sense of community, which finally is believed to lead to well-being. The data from each setting suggests that the relationship between spirituality and well-being operates differently at different settings; the path model, drawing on aggregate data, is still powerful enough to demonstrate the intricate relationship between the various kinds of variables, whether spiritual, contextual or well-being. 
All the survey findings demonstrate that the outlook of spirituality in different religious settings can be richly diverse, if not intricate. In order not to oversimplify the understanding of the phenomena to a few variables and a linear fashion, qualitative study was used in parallel, not just to situate and inform our interpretation of the survey data, but also to enhance our understanding of the underlying mechanism running in each setting.

The qualitative study, using participant observation, interviews and documentary analysis, helped uncover the operations in various settings. The activity setting framework offers five key dimensions (personnel, tasks, scripts, cultural values and belief, and motivation, Gallimore, et al., 1993) along which the researcher was able to unpack various religious settings and to identify how the social and eco-cultural environment in different settings influences daily routine and the development of different well-being outcomes. Activity settings provide members with a goal or motivation to strive for. The situated cultural values or belief substantiates various types of tasks and activities, scripts and tacit rules, or interpersonal relationships. The synergy maintains and sustains, both at the ideational or material level, various kind of patterns behaviour and psychological outcomes.

In this study, the charismatic church (CHC) emphasizes encountering God directly and experientially to promote and develop an emotionally rich environment, substantiated by praise and worship and other revival activities. An expressive, spontaneous and uplifting environment, as well as supportive interpersonal relationships, maintain an engaging environment in which people seek their faith. Members in such an emotionally rich environment can become more positive in an emotional sense, and psychological resiliency can be enhanced.

In contrast, the social concern church develops a very unique religious tradition which is social and action oriented. It supports various kinds of social and politically oriented and consciousness raising activities, maintained by a tacit communication style. It has more concern for the social over personal matters, and relatively distant interpersonal relationship (weak ties). Most importantly, this unique tradition is grounded on open and critical understanding and reflection on religious faith. Consequently, such a socially and actionoriented religious environment facilitates a high level of civic participation in members. Other-serving rather than self-serving attitudes help develop the life satisfaction for members,

The fundamentalist church is at a crossroads. The emphasis on the pre-eminence of truth above everything is the legacy passed down from its mother church with a Brethren 
tradition. However, the new generations in this branch seeding church provides more space for alternative interpretation of the traditionally fully embraced religious belief and practices, leaving room for change. Nevertheless, the closely-tied family type activities, plain and straightforward interactional patterns, and close and sincere interpersonal relationships inherited from the past seem to successfully shift the focus of fidelity from a hard and demanding religious ideal to a more humane commitment to religious community. As a result, members develop an ambivalent feeling, sometimes associated with guilty feelings, towards religious faith. Fortunately, in such a cohesive community church, members also develop strong bonding and interpersonal trust towards each other, believed to have a salutary effect on their trust towards people outside their church.

In contrast to the other churches, the evangelical church is a mega-church with several thousand congregants. Due to its size and resources, its focus on religious education and evangelical mission work is amplified. Nevertheless, also due to its church size, the evangelical church has developed a rather structured and formal, and less intimate, interactional pattern between members. Participation roles provided for members are also hindered by many people with ability and the relatively stricter requirements of people who want to serve. Members enjoy rich resources and professionally-led religious service that may enhance spiritual well-being, but members' sense of community and uniqueness has been compromised, if not sacrificed.

Qualitative study, using activity setting analysis, provides rich accounts of each setting by which to understand the uniqueness of each church, and also offers several key dimensions to further examine the relationship between the settings and well-being variables across the various settings. The five key dimensions - personnel, tasks, scripts, cultural values and belief, and motivation - are useful, heuristic and avoid oversimplified contrasts between the objective and subjective, material and ideational domain (Hays, 1994). Instead, they provide information in both subjective and objective realms, as well as on the ideational and material levels of the setting, necessary to make sense of the person in context. Moreover, the qualitative study demonstrates that religion provides people with different activity settings which facilitate different kinds of well-being and different pathways to the same kind of well-being. 


\subsection{Contributions and Implications of the study}

The major contributions of the present study can be discussed in three aspects: the importance of religion on well-being and community life; the importance of social context on well-being; and the benefit of mixed methodology in investigating well-being.

\subsubsection{The importance of religion on people's well-being}

Some have argued that the role of religion in secular society is declining; however religion is still robust (Peter L. Berger, 1967; Emmons \& Paloutzian, 2003) if not reviving (Dallmayr, 2003; Thomas, 2003) in many places in the world and has shown no sign of going away (Ebaugh, 2002; Emmons \& Paloutzian, 2003; Pargament \& Maton, 2000). Nevertheless, a lack of interest in (if not an active rejection of) religion is reflected in the literature and research. In recent decades rigorous empirical research on the influence of religion on wellbeing has been undertaken. Despite having impressive results, the necessity and value of studying religion are still sometimes raised (e.g., Funder, 2002). Following pioneering work in the field of psychology of religion (Emmons \& Paloutzian, 2003; Gorsuch, 1988; Pargament, 1997; Pargament \& Maton, 2000), the author of the present thesis supports the notion that religion is important for well-being and community life, and notes that psychological research has to take religion more seriously. It is especially important to two areas of psychological research: well-being and community.

\subsubsection{Religion and well-being}

The present thesis demonstrates the importance of religion on well-being. The survey findings show that the influence of religion on well-being can be through either spirituality and/or religious community. The MANOVA findings show that the influence of religion on well-being is small, but still significant. Path analyses suggests that the influence of religion on well-being works in a complex way. Religious influences well-being both directly and indirectly, but the influence can also be both positive and negative. In the present study, the influence of spirituality on well-being can be either unique or mediated through other variables: mental health, sense of community and voluntary organization membership. Experiential and behavioural aspects of religion normally have a positive influence, but fundamentalist religious beliefs can have a negative effect on civic participation and voluntary organization membership. The issue is complicated by the fact that while 
fundamentalist belief has a negative influence on civic participation, it has a significantly positive influence on mental health, enhancing life satisfaction.

We can see a multifaceted picture of the impact of religion on well-being. Regarding some well-being outcomes (life satisfaction, social trust, and mental health in the present study), religious influence is basically positive. As with other well-being outcomes (like civic participation in this study), certain aspects of religion can be positive while others are negative. What is not known in this study is whether or not religion is negative regarding some well-being outcomes. Therefore, when discussing the relationship between religion and well-being, it is very important to state clearly what kind of well-being and which aspect of religion are being discussed. Otherwise, the conclusions drawn can be misleading. However, in view of most previous research into well-being, primarily focusing on subjective wellbeing, the general account of the positive influences of religion is still valid.

The mediating effect of spirituality on well-being through sense of community, shown in the survey findings, is worthy of note. Eckersley (2007) argues that while individualism prevalent in western culture brings considerable cost to health and well-being, religion, with its social and collective focus, can play a significant role in restoring the situation. The present study supports this by showing that religion can enhance life satisfaction and social trust by building a stronger sense of community.

In addition, the qualitative study also demonstrates that differences in the mission and goals across religious settings can result in vast differences in spiritual outlooks, which are powerful in influencing human development (including well-being) in different ways. These findings accord with recent research on well-being, focusing on internal processes within the individual rather than just external, situational factors (Diener, et al., 1999). As Diener et al. understood, goals critically affect SWB. However, goals do not exist in a vacuum, but are deeply embedded in the socio-cultural environment. Moreover, Pargament and Maton (2000) point out that what psychologists can learn from religion is the ability to meet the basic human need for meaning. Empirical research corroborates the fact that meaning in life mediates the influence of religion on well-being or quality of life (Vilchinsky, 2005; Whitford \& Olver, 2009). Therefore, understanding the underlying religious and cultural meanings of settings is important to understanding the diverse manifestation of well-being in people. In recent studies regarding the psychology of religion, religion as a meaning system is highly regarded as a promising area of research (C. L. Park \& Paloutzian, 2005; Silberman, 
2005). Examining religion and well-being through the lens of meaning and goals will be fruitful.

Viewing the importance of religious goals and the multi-faceted picture of the religious impact on well-being, the vigour of spirituality in human everyday life is reaffirmed. If psychologists want to gain a better comprehension of well-being, understanding the various mechanisms of religious influence underpinning psychosocial and community life will be a feasible, and, the researcher believes, a fruitful area of study.

\subsubsection{Religion and community life}

The power of religion in the formation of community has long been recognized and documented (Durkheim, 1912/2001; Sarason, 1974), but research of community psychology focusing on religion or conducted in religious settings is still peripheral. Despite the research in religious settings in two special issues of the Journal of Community Psychology in the early twentieth first century (Kloos \& Moore, 2000a; Moore, et al., 2001), the interest in religion in relation to community research is still limited given the close relation between religion and the core values of community psychology, as Kloos and Moore (2000b) argue.

The present thesis extends its support for incorporating religion into community psychology research for two reasons. Firstly, the survey findings show that people in religious settings have a significantly stronger sense of community than their social centre counterparts. Moreover, in multiple regression and path models, the spirituality variables are highly associated with, and predictive of, sense of community. The causal effect of religion and sense of community can be reciprocal, and the findings in the present study clearly demonstrate that religion has a very unique role in the formation of community, as Durkheim argued nearly a century ago. If community psychologists would like to realize the overarching goal of the discipline (Sarason, 1974) -- fostering a sense of community -religious settings need to be a focus of research so that learning about the antecedents and the operation of sense of community can be furthered.

Secondly, the qualitative study further demonstrates that cultural values and the spiritual outlook of different religious settings can be a richly diverse area, even under the same religious traditions, implying that religion has multiple, distinctive and diverse ways of influencing people and community life. For community psychologists who take community as the basic unit of analysis and are interested in understanding persons in context, religion is 
one of the key areas that cannot be eschewed. In other words, unless we pay enough attention to the religious dimension of the various settings, the intricacy of the community life may not be easily understood.

Early in this century, in the special issue of Journal of Community Psychology, Kloos and Moore (2000b) and Hill (2000) proposed theoretical justifications to integrate spirituality into community psychology and the critical role of spiritual value in the community life. However, the present study provides empirical evidence for religion as an important factor in developing a sense of community and the distinctive cultural ethos of religious settings, which have huge impact on well-being. Supporting a sense of community and well-being is important in community psychology (Kloos \& Moore, 2000b; Toro, 2005), and therefore religion - one of the key predictors and associated factors - should gain proper focus.

Putting religion back into psychological research not only enables psychologists to learn more about human behaviour and underlying processes, but it is also an absolute necessity because of the integral role religion can play in human social and community life (Baumeister, 2002; Maton, 2001).

\subsubsection{The importance of social context on people's well-being}

In the study of health and well-being, most study have been conducted at the individual level while research on social determinants (Marmot \& Wilkinson, 1999) or the social origins (Eckersley, Dixon, \& Douglas, 2001) of health and well-being has only attracted research in the recent decade. The effect of the social environment on health and well-being has been mentioned and a disproportionate number of studies into well-being focus on the individual level and subjective realm which seems to suggest that well-being is largely individual, with less value attached to concern about the social environment. Likewise, although empirical research on the relationship between religion and health and well-being has made much progress in the past decade, with generally positive results, the religiouslybased context has also been rarely examined and explored in detail. This, unfortunately, seems to suggest that spirituality can be understood in isolation from the religious context. However, this is not the stance taken in the present study. Instead, both the survey and qualitative study clearly demonstrate the importance of examining context related to social capital, and through the cultural environment of the settings. 


\subsubsection{Social capital and well-being}

Our survey findings indicate the critical role of contextual factors - sense of community - in well-being outcomes (life satisfaction and social trust), but interpersonal well-being (social trust) was also shown, in the path model, to be significant in affecting well-being at the individual level (life satisfaction). These findings convince us of the claims that social capital matters in health and well-being (Baum, 1999; Helliwell \& Putnam, 2004; Kawachi \& Berkman, 2000; Marmot \& Wilkinson, 1999; Kwame McKenzie \& Harpham, 2006a; K. Mckenzie, Whitley, \& Weich, 2002). Although the idea of social capital my need more refinement, a large number of studies point out that social relations and networks have psychological implications. Social capital (especially in terms of trust), not only has an inverse association with mental illness (De Silva, Mckenzie, Harpham, \& Huttly, 2005), but also has a beneficial effect on health and well-being (Kawachi \& Berkman, 2000; K. Mckenzie, et al., 2002). The salutary effect of social capital is not only on the individual level, but also the community level (Sampson, 1996).

This study clearly demonstrates the point that feelings of a sense of community have a strong influence on life satisfaction and social trust, which can further strengthen subjective well-being at the individual level. Although some may argue that the sense of community can be double-edged, not only enhancing intimacy within a group, but also creating exclusion towards outsiders (Baum, 1999; Fisher \& Sonn, 2007), the present study suggests that enhancing sense of community towards immediate settings will not decrease social trust in general or towards people outside immediate settings, rather the opposite. It may reflect the specific benefits of nurturing a sense of community within the settings in the present study.

\subsubsection{Cultural environment and well-being}

Besides sense of community and social capital, the qualitative study provides a more holistic and in-depth picture that can capture the nuances of the religious context. It seems to suggest the critical role of cultural environment of the settings on well-being. The activity setting analysis reveals that various religious settings develop a unique and intriguing social and cultural environment, in which members develop distinctive behaviour patterns, tacit communication, as well as interactional and relational styles. These distinctive social environments can enhance well-being outcomes, manifested in life satisfaction, social trust, civic participation and sense of community. 
Eckersley et al. (2001) argues, regarding the social determinants of health, that research has unduly focused on socio-economic factors at the expense of other social sources of health and well-being, including historical, global, cultural or organizational dimensions. The author of the present thesis supports argument, and notes that research into religion and health and well-being has unduly neglected the religious setting as well as the cultural environment of the religious settings, in favour of other individual factors in the subjective religious domain.

Nevertheless, it is fair to say that examining religious and cultural context is not an easy task. While we will discuss in more detail the methodological challenge of examining culture in the following section (14.2.3), we will highlight some of advance in psychology regarding culture and its relation to well-being. Psychologists have been always criticised, either internally or externally, for the lack of concern for culture (Greenfield, 2000), or not taking the culture seriously (Cole, 1996), or for being too naïve and ill-prepared to understand the issues of culture (Schwartz, 1981). Global demographic and socio-economic change and massive immigration (Cooper \& Denner, 1998), the importance of the cultural aspects of human development (Rogoff, 1990, 2003), and culture and cognition (DiMaggio, 1997) have brought the culture back under the closer scrutiny of psychologists, resulting in fruitful exchange between the two areas.

Although cross-cultural psychologists try to tease out the underlying common factors between cultures, such as individualism versus collectivism (Triandis, 1995), independent and interdependent views of the self (H. R. Markus \& Kitayama, 1991), other cultural psychologists (ecocultural, cultural-historical, or sociocultural psychologists), such as Cole (1996), Rogoff (2003) or Ratner (2006), regard this kind of understanding of culture as too superficial to be at all meaningful. Instead they consider culture and psychology to be organically interrelated and intertwined with each other. The difference between the two camps can be understood in several major dimensions. Firstly, while cross-cultural psychological approach often regards culture as a variable (Greenfield, 2000; such as individualism or collectivism, proposed by Triandis, 1995), the socio-cultural approach regards culture as a resource of toolkits, where people can draw ideas, emotions and various kinds of practices (Cole, 1996). Secondly, while the cross-cultural approach emphasizes the ideational side of culture and conceptualizes culture as core societal values (Cooper \& Denner, 1998), the socio-cultural approach highlights both the symbolic and the materialistic 
sides of the cultural environment and the mediated effect of culture through physical artefacts and practical activity (Cole, 1996). Thirdly, while the cross-cultural approach considers culture to be a separate entity influencing individuals externally, the socio-cultural approach understands the individual and cultural processes as mutually constitutive, rather than separate from each other (Cole, 1996; Rogoff, 2003). In the ongoing participation process, people not only draw on the cultural tools and resources provided by the cultural environment, thereby being shaped and constrained by them, but also actively contribute to the creation of the psychological and cultural processes of which they are a part.

Cross-cultural and cultural psychology represents attempts to empirically understand how broader cultural environment influences the individual. However, differences between cross-cultural and cultural psychology raise a very important issue - can culture be separated from social structure? Although culture is normally regarded as ideational, many argue that it cannot be understood in isolation from the material world (Cole, 1996), or socio-material reality (Alvesson, 2002). Hays (1994), in her paper Structure and Agency and the Sticky Problem of Culture, discusses in detail how culture is tied closely with social structure and agency, and a simple contrast between the concepts is misleading. To really understand the cultural environment of a setting, elements in the structural dimension cannot be ignored. That is also why tasks, activities, and cultural scripts of the settings have been proposed (Alvesson, 2002; Cole, 1996) and are also explored in the present study, along with cultural belief.

Pargament and Maton (2000) suggest that, when examining religious institutions, researchers can investigate several dimensions: theology, mission and organizational structure. When combined with the religious influence - either externally to society or internally on members - it results in richly intricate patterns and forms of religious settings. Despite using different dimensions, their suggestion is echoed by five key features suggested in the present study, and both ideational and material dimensions of the religious settings should also be examined. In addition, along with Pargament and Maton’s argument, the different combinations and synergies of the key dimensions in the religious settings result in diversity in forms and functions of religion.

In their work expanding behaviour setting theory, Luke, Rappaport and Seidman (1991) propose the use of setting phenotypes as well as Barker's (1968) notion of genotype in examining people's behaviour context. As they understand it, the genotype notion is 
concerned with various forms of the setting, and the phenotype concept is better suited to examining different functions served by the settings given the same form of the setting. After studying 510 meetings of 13 mutual help groups, they discovered four basic behavioural patterns: personal, impersonal, small talk, and advising. As they argue, using both the genotype and phenotype concept can enable researchers to maintain a balance between the structure and function in understanding the setting.

This present study also demonstrates this close linkage between the structural and cultural dimensions of a setting. For example, the evangelical church, because of its size, has developed a unique cultural environment that is associated with rich resources, formality, and obedience, which is also aligned with bureaucracy. In contrast, the social concern church, because of its strong socially concerned ethos, has evolved many activities, artefacts or scripts which accord with its goals.

Whether or not the culture can be separated from the social structure, the influence of culture on health and well-being has been acknowledged (Eckersley, 2001, 2007). In his examination of young people's health and well-being in Australia, Eckersley (2001) emphasizes the critical impact of cultural values. He notes that western culture (represented by its individualization and modernization) can amplify or moderate the effects of socioeconomic factors (such as inequality) on health and well-being, but also can act in independent ways. Similarly, in the present study, we can also see the impact of distinctive culture in the religious setting on various kinds of well-being. Different religious traditions, with unique cultural values and goals, develop quite distinctive cultural environments, which finally moderate individual development and well-being. Therefore, the present study demonstrates that to fully understand the mechanism of well-being in general, or the relation between religion and well-being in particular, the immediate settings, including structural and cultural dimensions, cannot be overlooked; otherwise, the richly diverse connections will be missed.

In addition to putting religion back into psychological research, bringing back context is not just required in fields like epidemiology (Diez-Roux, 1998), but also in psychology, especially research on well-being. Recently, pioneers of well-being research, such as Diener (2008, 2009) and Myers (2007), support the exploration of the context of well-being and building on the "third pillar" of positive psychology: positive communities/institutions. This study, echoing their calls, contributes to understanding in this aspect. Nevertheless, as 
mentioned earlier, examining social context is a challenging, if not daunting, task. That brings us to our last point: the importance of mixed methodology.

\subsubsection{The importance of mixed methodology}

Given the importance of context in well-being research, examining social context is not an easy task. As argued in Chapter Three, context is a slippery concept because there is diversity of contexts - immediate settings, institution or organization, community, society or cultural environment. Moreover, when we examine the context, it is not clear whether the objective versus subjective or structural versus cultural dimensions should be the focus. What further complicates the issue, as Hays (1994) argues, is the difficulty in separating culture and social structures. As to untangling the two, if they are inherently intertwined with each other, and how to empirically study them are the real challenges facing researchers. Structure and culture are elusive concepts, and can be unfamiliar to psychologists.

As argued previously, to really understand the context of well-being in general, or the relationship between religious setting and well-being in particular, researchers have to delve into the sticky problems of culture, structure and agency (Hays, 1994). While questionnaires are notoriously ill-equipped for capturing the nuances and detail in subjective experiences, or in the collective meaning of culture, other kinds of methodologies should be explored to avoid narrowness or even distortions in research. The present thesis proposes an attempt that combines both survey and field work study, in the hope of overcoming limitations in the conventional scientific approach and to make full use of the strength of the qualitative approach to provide a richer and more holistic account of the studied phenomenon.

While the survey provides us with a snapshot and the 'hard facts' of the phenomenon, field study enables researchers to explore religious setting in more detail. Interviews help the author to uncover the church members' subjective thoughts and experiences in the settings, and participant observation and prolonged engagement in the settings enabled him to obtain first hand experience and an insider perspective of the dynamics and culture of the specific setting, which can be compared with the other sources of data and used to inform later analysis and interpretation.

As well as the unique benefits inherent in each method, the mixing of qualitative and quantitative methods also provides additional advantages derived from their parallel use. According to Greene and her colleagues (Greene, 2007; Greene, Careacelli, \& Graham, 1989), 
the combination of qualitative and quantitative methods can boost understanding of the topic of research by achieving triangulation, complementarity, initiation and expansion.

For example, the primary goal of using the field study in parallel with the survey in the present study was to extend the scope and breadth of inquiry, especially related to the exploration of the nuances of the religious context. While this kind of mixed method practice is very common in evaluation studies, in which quantitative methods evaluate program outcomes and qualitative methods assess program implementation (Greene, 2007), it also accords very well with the present study to expand the understanding of the aspects (social, cultural, relational and material) of religious settings through the use of different methods. The survey findings provide us hard factual data about social capital within settings (sense of community, social trust and civic participation), and the field study furthers understanding of the cultural ethos and the interpersonal dynamic of religious settings, difficult to measure by using surveys alone. The mixture of methods thus expands our scope and ability to capture the key elements of the context which are elusive and dynamic.

The second benefit of employing mixed methods in the present study is triangulation. In its general use (Denzin, 1978; Greene, 2007), triangulation refers to the convergence of results, regarding the same phenomenon, through multiple methods or data source. In this study, one of the major goals is to examine the beneficial effects of religion on well-being, in terms of life satisfaction, social trust and civic participation. The quantitative survey demonstrates that the influence of religion on well-being is small, but still significant, and that not all religious settings have the same kinds of effects. Findings from the qualitative study corroborate the picture that each setting has a quite distinctive culture believed to enhance the specific dimension of well-being.

In addition, even though some interviews with members of two churches (social concern and evangelical church) indicate that the sense of belonging to the church is not satisfactory or less than expected, the survey findings, despite corroborating the interviews' relatively lower results compared to the other two churches, still show a significant and stronger sense of community than their social centre counterparts. The mixed method components in the present study make our claim stronger and more substantial.

The third benefit provided by mixed methods in the present study is complementarity. According to Greene (2007), mixed method study with this focus "seeks broader, deeper, and more comprehensive social understandings by using methods that tap into different facets or 
dimensions of the same complex phenomenon” (p.101). In the present study, realizing that well-being, religious phenomenon and religious settings are all complex and multifaceted, complementing the survey with the more holistic field study can provide both individual (subjective data) and collective perspectives that can help interpret the survey findings.

Another advantage of using mixed methods in this study is initiation. To Greene (Greene, 2007), the most exciting thing about mixing methods is the generative potential of the divergent or even contradicting results shown in different methods, which she argues results in fresh insights, perspectives and understanding. In our study, the survey findings show that SCC church members are significantly lower in their spirituality variables than their counterparts in the other religious settings, whether in relation to religious belief, experience, or practices or involvement in the church. At first sight, this seems to suggest that SCC members are not as spiritual as the members in other religious settings. Nevertheless, our qualitative study findings (especially in interviews) reveal that members of SCC are very strong and persistent in their religious faith and action. The not fully compatible results of qualitative and quantitative studies force researchers to engage in understanding the difference (Greene, 2007) in different religious settings, rather than simply viewing the issue in a linear fashion. In other words, it is not the SCC members who are not spiritual enough, but the questionnaires which are not able to capture the diverse and complex nuance of the religious faith. In this case, without the help of alternative methods, the understanding of the studied phenomenon may be at risk.

In summary, the goal of examining the impact of religious communities on well-being poses a methodological challenge to the present study because of the sticky problem of context. Due to the intertwined relationship between social structure, culture and human agency, conventional survey methodology is not well-equipped to capture the nuances and diverse meanings within or across various settings. Multiple methods, however, enable the researcher to unpack and uncover the detailed, if not intricate, dynamics running in each setting. In addition, multiple methods help triangulate, complement, and expand the findings provided by each method so that stronger claims can be made. Furthermore, the diverse if not contradictory findings, also gave the researcher a new perspective and understanding that a single method cannot provide. 


\subsection{Strengths and limitations of the study}

\subsubsection{Limitations}

Despite the benefits provided by using mixed methods, this study also has limitations, which can be addressed in later studies. In the survey section, the use of crosssectional and correlational design has been commonly criticized in psychological research for its inability to provide causal relationships. As it make sense to conclude that religious people tend to have higher level of satisfaction in life and a greater degree of social trust, it is also feasible to contend that people who have a higher level of life satisfaction and trust like to attend religious activity to nurture their religiosity. While it is reasonable to assume that religious setting strongly influences religious belief and behaviour, it is also logical to argue that people also actively seek the kind of church with accords with their own thoughts and beliefs. Moreover, the variety of age ranges in each setting should be noted (especially the high numbers of young adults - aged 18 - in the social centre sample). Furthermore, the limited sample sizes in each setting deprive the researcher of the ability to conduct separate path analysis in each setting, so that different mechanisms underlying each setting can be uncovered. Furthermore, even though the four religious settings represent the four major distinctive Protestant spiritual traditions in Hong Kong, whether they can represent all the varieties of Protestant tradition in over 1200 congregations in Hong Kong (Wu \& Fok, 2006) still awaits further examination, especially when the present study puts so much emphasis on the importance of examining the context of a studied phenomenon. In addition, given the importance and critical role of spirituality on well-being and community life, the understanding and measurement of spirituality is far from easy. The survey section demonstrates many ways spirituality can influence well-being, and the qualitative section shows the nuance and intricacy, if not abundance, of religious meaning, experience, and behaviour in various religious settings; this plethora is not fully captured by survey measurement. For example, fundamentalist belief certainly is not the only way to understand religious beliefs in our studied settings because we will miss the very rich social concern meaning attached to their religious faith by the SCC person. Even within the fundamentalist church, the fundamentalist scale cannot capture the ambivalence of people who are reflecting truly and deeply about their religious faith. While acknowledging that quantitative measurement employed in the present investigation can provide a window to understand 
religious faith, the studied dimensions should be expanded and complemented by qualitative research so as to identify the relevant aspects to be investigated and to ensure the measurement used is culturally anchored.

In the qualitative section there are also limitations. One of the common criticisms about qualitative methods is the validity and reliability issue. Instead of making an authoritative claim of objective truth, the author acknowledges the interpretative nature as well as the impossible subjective judgment that has been made in the process. Nevertheless, the author tried to lay bare the inquiry process, and to separate the description and the interpretation of the findings, so that the readers can make their own interpretation and explanation and the public discourse can continue. In addition, despite the richness of the activity setting findings, this study still does not give the whole picture of the studied phenomenon in two dimensions. Firstly, microscopically, heterogeneity within each setting has not been studied in detail. As described in brief in the findings, but not explored in detail in the discussion section, there are some discrepancies and potential heterogeneity in religious beliefs and practices between people of different age and different camps within each setting. Therefore, the amount of heterogeneity and how settings address the issues of diversity would be worth further exploring in future study. Secondly, macroscopically, as argued in Chapter Three, each organization is not a closed system, but an open system interacting with the broader ecological environment. Activity settings, no doubt, are a good analytical tool to understand the internal dynamics of the immediate settings, but how this setting is influenced by the external broader environment, or even the socio-political realm, is still not examined thoroughly. As briefly mentioned in Chapter Six, the spiritual orientation of Protestant churches in Hong Kong shifted from a more balanced point of view to a more conservative and fundamentalist orientation from the 1950s, the post-war era. Why did this happen and how does it relate to the socio-political context? Therefore, when examining the well-being and its relation to religion, can the macro social-political realm and the relevant analysis tool be neglected?

\subsubsection{Strengths}

Despite some limitations, this study still has its merits and contributions to make. Firstly, the multi-dimensional conceptualization of spirituality and well-being enables the researcher to discriminate between which aspects of spirituality are mostly associated with 
which aspects of well-being. In this study, different aspects of spirituality cannot be easily teased out in relation to satisfaction with life and social trust, but the effects of spirituality (related to religion) on civic participation were observed. In addition, the complex direct and indirect effects, as well as the positive and negative effects of religion on different kinds of well-being outcomes helps delineate the intricate relationships between religion and wellbeing. As Koenig et al. (2000) and Pargament (2002a) note, religion can be sweet and bitter and the salutary effects of various forms of religion may be different in terms of well-being. Secondly, instead of just focusing on the individual factors and variables, as many psychologists do, this study takes the ecological issue seriously. Not only were contextual factors examined in the survey section, but people's cultural and contextual environments were studied in the qualitative section, enabling a more holistic and nuanced understanding of the phenomenon. Finally, combining both survey and qualitative findings, this study demonstrates that well-being is not only associated with religion, but also closely intertwined with both private and public facets of religion, for its ability to address the issue of context.

\subsection{Future Research}

Research into religion and well-being is thriving. The author has some suggestions for worthwhile research. Firstly, the present study focuses on the Asian population, and explores Judeo-Christian traditions. Does the effect of religion on well-being (on the individual level or interpersonal level) still hold in other Chinese religion? Secondly, the Protestant tradition is institutionalized, and so can other Chinese religions (such as Daoism, which is be more loosely organized) similarly build a sense of community? Thirdly, as well as subjective well-being, the relationship between religion and other human strengths, or collective well-being, can be explored. A study of religion and social capital (Smidt, 2003), or the notion of spiritual capital (P. L. Berger \& Hefner, 2003; Finke, 2003; Malloch, 2003; Verter, 2003; Woodberry, 2003) constitute some research into the impact of religion beyond the individual level. Fourthly, regarding the mechanism underlying religion and well-being, meaning, goals, and optimism are some of the most researched linkages. The present study proposes that sense of community is a worthwhile and valuable pathway related to religion and well-being. Whatever the line of research, the examination of religion and well-being is fascinating because of the numerous, relationships that can be revealed and their close relevance to human and community life. 


\section{Reference}

Adelmann, P. K. (1995). Emotional labour as a potential source of job stress. In S. L. Sauter \& L. R. Murphy (Eds.), Organizational risk factors for job stress. Washington, DC: American Psychological Association.

Allport, G. W., \& Ross, J. M. (1967). Personal religious orientation and prejudice. Journal of Personality and Social Psychology, 5, 432-443.

Altemeyer, B., \& Hunsberger, B. (1992). Authoritarianism, religious fundamentalism, quest, and prejudice. The International Journal for the Psychology of Religion, 2(2), 113133.

Altemeyer, B., \& Hunsberger, B. (2004). A revised religious fundamentalism scale: The short and sweet of it. The International Journal for the Psychology of Religion, 14(1), 4754.

Alvesson, M. (2002). Understanding organizational culture. London: SAGE.

Anderson, M. (2006). Community psychology, political efficacy and trust. Paper presented at the Annual Meeting of the Midwest Political Science Association. Chicago. April 2006.

Andrews, M., Sclater, S. D., Rustin, M., Squire, C., \& Treacher, A. (2000). Introduction. In M. Andrews (Ed.), Lines of narrative : psychosocial perspectives (pp. xiii, 202). London ; New York: Routledge.

Argyle, M. (2000). Psychology and religion: An introduction. London ; New York: Routledge.

Argyle, M. (2001). The psychology of happiness (2nd ed.). Hove, East Sussex: Routledge.

Atkinson, R. (1998). The life story interview. London: Sage Publications Ltd.

Axinn, W. G., \& Pearce, L. D. (2006). Mixed method data collection strategies. New York ; Cambridge: Cambridge University Press.

Baker, H. D. R. (1983). Life in the cities: The emergence of Hong Kong man. The China Quarterly, 95, 469-479.

Banyard, V. L., \& Miller, K. E. (1998). The powerful potential of qualitative research for community psychology American Journal of Community Psychology, 26(4), 485-504.

Barker, R. G. (1968). Ecological psychology: Concepts and methods for studying the environment of human behaviour. Stanford, CA: Stanford University Press.

Barker, R. G., \& Schoggen, P. (1973). Qualities of community life (1st ed.). San Francisco: Jossey-Bass.

Baron, R. M., \& Kenny, D. A. (1986). The moderator-mediator variable distinction in social psychological research: Conceptual, strategic, and statistical considerations. Journal of Personality and Social Psychology, 51(6), 1173-1182.

Batson, C. D. (1998). Altruism and prosocial behavior. In D. T. Gilbert, S. T. Fiske \& L. Gardner (Eds.), The handbook of social psychology (Vol. 2, pp. 915-981). Boston: McGraw-Hill

Batson, C. D., Ahmad, N., \& Lishner, D. A. (2009). Empathy and altruism. In C. R. Snyder \& S. J. Lopez (Eds.), Oxford handbook of positive psychology (2nd ed.). Oxford ; New York: Oxford University Press.

Batson, C. D., Schoenrade, P., \& Ventis, W. L. (1993). Religion and the individual New York: Oxford University Press.

Batson, C. D., \& Ventis, W. L. (1982). The religious experience: A social-psychological perspective. New York: Oxford University Press. 
Baum, F. (1999). Social capital: is it good for your health? Issues for a public health agenda. Journal of Epidemiology Community Health, 53, 195-196.

Baumeister, R. F. (2002). Religion and psychology: Introduction to the special issue. Psychological Inquiry, 13(3), 165-167.

Baumeister, R. F., \& Leary, M. R. (1995). The need to belong: Desire for interpersonal attachment as a fundamental human motivation. Psychological Bulletin, 117, 497-529.

Becker, P. E. (1999). Congregations in conflict: Cultural models of local religious life. Cambridge ; New York: Cambridge University Press.

Belzen, J. A. (1997). The historicocultural approach in the psychology of religion: Perspectives for interdisciplinary research. Journal for the Scientific study of religion, 36(3), 358-371.

Bension, P. L., Roehlkepartain, E. C., \& Rude, S. P. (2003). Spiritual development in childhood and adolescence: Toward a field of inquiry. Applied Developmental Science, 7, 204-212.

Berger, P. L. (1967). The sacred canopy: Elements of a sociological theory of religion. Garden City, N.Y.: Doubleday.

Berger, P. L., \& Hefner, R. W. (2003). Spiritual capital in comparative perspective. Retrieved June 112006 from http://www.spiritualcapitalresearchprogram.com/pdf/Berger.pdf.

Berger, P. L., \& Neuhaus, R. J. (1977). To empower people. Washington, D. C.: American Enterprise Institute.

Berkman, L. F., \& Glass, T. (2000). Social integration, social network, social support, and health. In I. Kawachi \& L. F. Berkman (Eds.), Social epidemiology (pp. 137-173). New York ; Oxford: Oxford University Press.

Bertalanffy, L. v. (1968). General system theory: foundations, development, applications (Rev. ed.). New York: G. Braziller.

Blumer, H. (1969). Symbolic interactionism : Perspective and method. Englewood Cliffs, N.J.: Prentice-Hall.

Bolman, L. G., \& Deal, T. E. (2003). Reframing organizations : Artistry, choice, and leadership (3rd ed.). San Francisco: Jossey-Bass.

Bottoms, B. L., Shaver, P. R., Goodman, G. S., \& Qin, J. (1995). In the name of God: A profile of religion-related child abuse Journal of Social Issues, 51(2), 85-111.

Boulding, K. E. (1956). General systems theory: The skeleton of science. Management science, 2(3), 197-208.

Bourdieu, P. (1977). Outline of a theory of practice. Cambridge ; New York: Cambridge University Press.

Bourdieu, P. (1986). The forms of capital. In J. Richardson (Ed.), Handbook of Theory and Research for the Sociology of Education (pp. 241-258). New York: Greenwoood.

Bowling, A. (1997). Measuring health : A review of quality of life measurement scales (2nd ed.). Buckingham ; Philadelphia: Open University Press.

Brodsky, A. E. (1996). Resilient single mothers in risky neighbourhoods: Negative psychological sense of community. Journal of Community Psychology, 24, 347-363.

Brodsky, A. E., O' Campo, P. J., \& Aronson, R. E. (1999). PSOC in community context: Multi-level correlates of a measure of psychological sense of community in low income, urban, neighbourhoods. Journal of Community Psychology, 27, 659-679.

Bronfenbrenner, U. (1979). The ecology of human development: Experiments by nature and design. Cambridge, Mass: Harvard University Press. 
Bronfenbrenner, U. (1995). Developmental ecology through space and time: A future perspective. In P. Moen, G. H. Elder, K. Lüscher \& U. Bronfenbrenner (Eds.), Examining lives in context : Perspectives on the ecology of human development (1st ed. ed.). Washington, DC: American Psychological Association.

Bruner, J. S. (1990). Acts of meaning. Cambridge, Mass.: Harvard University Press.

Buckley, W. F. (1967). Sociology and modern systems theory. Englewood Cliffs, N. J.: Prentice-Hall.

Byrne, D. (2002). Interpreting quantitative data. London: SAGE Publications.

Cahill, S. (2004). Inside social life : readings in sociological psychology and microsociology (4th ed.). Los Angeles: Roxbury Pub.

Calhoun-Brown, A. (2005). Be fruitful and multiply? Church size and the generation of social capital. Paper presented at the 2005 Meeting of the American Political Science Association, Washington, DC, September 1-4 2005.

Cantor, N., \& Sanderson, C. A. (1999). Life task participation and well-being: The importance of taking part in daily life. In D. Kahneman, E. Diener \& N. Schwarz (Eds.), Well-being: The foundations of hedonic psychology. New York: Russell Sage Foundation.

Carroll, J. W., \& Roozen, D. A. (1990). Congregational identities in the Presbyterian church. Review of Religious Research, 31(4), 351-369.

Chan, J. M. (2005). Hong Kong style "people power": The mode of social mobilization and the reconfiguration of public opinion politics. In C. H. Ng, T. L. Lui \& E. Ma (Eds.), Hong Kong style cultural studies. Hong Kong: Hong Kong University Press (in Chinese).

Chan, S. H. (1999). The three model of Hong Kong church state relationship (Chinese). Si, 65, 4-8.

Chan, S. H. (2002). The structure and change of religion. In T. Tse, K. C. (Ed.), Our place, our time: A new introduction to Hong Kong society (pp. 375-410). Hong Kong Oxford Unviersity Press (in Chinese).

Chavis, D. M., \& Wandersman, A. (1990). Sense of community in the urban environment: A catalyst for participation and community development. American Journal of Community Psychology, 18(1), 55-81.

Cheal, D. (2005). Dimensions of sociological theory. New York: Palgrave Macmillan.

Chen, S. X., Bond, M. H., \& Cheung, F. M. (2006). Personality correlates of social axioms: Are beliefs nested within personality? Personality and Individual Differences, 40, 509-519.

Chow, W. Y. (2001). A response to "The social roles of Hong Kong churches after the return to Chinese sovereignty in 1997". Journal of History of Christianity in Modern China, 4, 71-80.

Cole, M. (1996). Cultural psychology: A once and future discipline. Cambridge, Mass.: Belknap Press of Harvard University Press.

Cole, M. (2003). Vygotsky and context: Where did the connection come from and what difference does it make? Paper presented at the Biennial conference of the International Society for Theoretical Psychology.

Cole, M., \& Scribner, S. (1978). Introduction. In L. S. Vygotsky \& M. Cole (Eds.), Mind in society: the development of higher psychological processes. Cambridge: Harvard University Press. 
Cole, M., \& Wertsch, J. V. (2002). Beyond the Individual-Social Antimony in Discussions of Piaget and Vygotsky Retrieved 31 Oct 2006, from http://www.massey.ac.nz/ alock/virtual/colevyg.htm

Coleman, J. S. (1988). Social capital in the creation of human capital. American Journal of Sociology, 94, 95-120.

Collins, R., \& Collins, R. (1994). Four sociological traditions. New York: Oxford University Press.

Cooper, C. R., \& Denner, J. (1998). Theories linking culture and psychology: Universal and community-specific processes. Annual Review of Psychology, 49, 559-584.

Costanza, R., Fisher, B., Ali, S. B., C., Bond, L., Boumans, R., \& Danigelis, N. L. (2007). Quality of life: An approach integrating opportunities, human needs, and subjective well-being. Ecological Economics 61, 267-276.

Cowen, E. L. (1991). In pursuit of wellness. American Psychologist, 46(4), 404-408.

Cowen, E. L. (2000). Community psychology and routes to psychological wellness. In J. Rappaport \& E. Seidman (Eds.), Handbook of community psychology (pp. 79-99). New York: Kluwer Academic/Plenum.

Creswell, J. W. (2003). Research design: Qualitative, quantitative, and mixed methods approaches (2nd ed.). Thousand Oaks, CA.: Sage Publications.

Creswell, J. W., Clark, V. L. P., Gutmann, M. L., \& Hanson, W. E. (2003). Advanced mixed methods research designs. In C. Teddlie \& A. Tashakkori (Eds.), Handbook of mixed methods in social \& behavioural research. Thousand Oaks, CA.: SAGE Publications.

Creswell, J. W., \& Miller, D. L. (2000). Determining validity in qualitative Inquiry. Theory into Practice, 39(3), 124-130.

Csikszentmihalyi, M. (1990). Flow : The psychology of optimal experience (1st ed.). New York: Harper \& Row.

Cummins, R. A. (1997). Comprehensive quality of life scale - Adult. Melbourne: School of Psychology, Deakin University.

Davidson, W. B., \& Cotter, P., R. (1989). Sense of community and political participation. Journal of Community Psychology, 17, 119-125.

Davidson, W. B., \& Cotter, P., R. (1991). The relationship between sense of community and subjective wellbeing: A first look. Journal of Community Psychology, 19, 246-253.

De Silva, M. J., Mckenzie, K., Harpham, T., \& Huttly, S. R. (2005). Social capital and mental illness: A systematic review. Journal of Epidemiology Community Health, 59, 619627.

Delhey, J., \& Newton, K. (2003). Who Trust? The origins of social trust in seven countries. European Societies, 5(2), 93-137.

Denzin, N. K. (1978). The research act : A theoretical introduction to sociological methods. New York: McGraw-Hill.

Diener, E. (1984). Subjective well-being. Psychological Bulletin, 95(3), 542-575.

Diener, E. (2008). Myths in the Science of Happiness, and Directions for Future Research. In M. Eid \& R. J. Larsen (Eds.), The science of subjective well-being (pp. 493-514). New York: Guilford Press.

Diener, E. (2009). Positive psychology: Past, present, and future. In C. R. Snyder \& S. J. Lopez (Eds.), Oxford handbook of positive psychology (2nd ed., pp. 7-12). Oxford ; New York: Oxford University Press.

Diener, E., Emmons, R. A., Larsen, R. J., \& Griffen, S. (1985). The satisfaction with life scale. Journal of Personality Assessment, 49, 71-75. 
Diener, E., Sapyta, J. J., \& Suh, E. (1998). Subjective well-being is essential to well-being. Psychological Inquiry, 9, 33-37.

Diener, E., \& Suh, E. (1997). Measuring quality of life: Economic, social, and subjective indicators. Social Indicators Research, 40, 189-216.

Diener, E., \& Suh, E. M. (1999). National differences in subjective well-being. In D. Kahneman, E. Diener \& N. Schwarz (Eds.), Well-being: The foundations of hedonic psychology. New York: Russell Sage Foundation.

Diener, E., \& Suh, E. M. (2000a). Culture and subjective well-being. Cambridge, Mass. ; London: MIT Press.

Diener, E., \& Suh, E. M. (2000b). Measuring subjective well-being to compare the quality of life of cultures. In E. Diener \& E. M. Suh (Eds.), Culture and subjective well-being. Cambridge, Mass. ; London: MIT Press.

Diener, E., Suh, E. M., Lucas, R. E., \& Smith, H. L. (1999). Subjective well-being: Three decades of progress. Psychological Bulletin, 125(2), 276-302.

Diez-Roux, A. V. (1998). Bringing context back into epidemiology: Variables and fallacies in multilevel analysis. American Journal of Public Health, 88(2), 216-222.

Diez-Roux, A. V. (2000). Multilevel analysis in public health research. Annual Review of Public Health, 21, 171-192.

DiMaggio, P. (1997). Culture and cognition. Annual Review of Sociology, 23, 263-287.

Dionne, E. J., \& Chen, M. H. (2001). Sacred places, civic purposes: Should government help faith-based charity? Washington, D.C.: Brookings Institution Press.

Dittes, J. E. (1969). Psychology of religion. In G. Lindzey (Ed.), Handbook of Social Psychology. Reading, Mass: Addison-Wesley.

Dokecki, P. R., Newbrough, J. R., \& O' Gorman, R. T. (2001). Toward a community-oriented action research framework for spirituality: Community psychological and theological perspectives. Journal of Community Psychology, 29(5), 497-518.

Dorr, D. (1984). Spirituality and justice. Dublin: Gill and Macmillan.

Dorr, D. (2004). Time for a change: A fresh look at spirituality, sexuality, globalisation, and the church. Blackrock, Co. Dublin: Columba Press.

Dudley, C. S., \& Johnson, S. A. (1993). Energizing the congregation: Images that shape your church's ministry (1st ed.). Louisville, KV.: Westminster/John Knox Press.

Durkheim, E. (1912/2001). The elementary forms of religious life. Oxford: Oxford University Press.

Durkheim, E. (1952). Suicide : A study in sociology. London: Routledge.

Ebaugh, H. R. (2002). Presidential address 2001 Return of the sacred: Reintegrating religion in the social science. Journal of the Scientific Study of Relgion, 41(3), 385-395.

Eckersley, R. (2001). Culture, health and well-being. In R. Eckersley, J. M. Dixon \& R. M. Douglas (Eds.), The social origins of health and well-being. Cambridge ; Melbourne: Cambridge University Press.

Eckersley, R. (2004). Well and Good. Melbourne: The Text Publishing Company.

Eckersley, R. (2007). Culture, spirituality, religion and health: Looking at the big picture. Medical Journal of Australia, 186(10), s54-56.

Eckersley, R., Dixon, J. M., \& Douglas, R. M. (2001). The social origins of health and wellbeing. Cambridge ; Melbourne: Cambridge University Press.

Eitel, E. J., \& Lethbridge, H. J. (1895/ 1983). Europe in China. Hong Kong: Oxford University Press. 
Eliade, M. (1961/ 1999). A new humanism. In R. T. McCutcheon (Ed.), The insider/outsider problem in the study of religion : a reader. London ; New York: Cassell.

Ellens, J. H. (2004). The destructive power of religion: violence in Judaism, Christianity, and Islam. Westport, Conn.: Praeger.

Ellison, C. G., \& Levin, J. S. (1998). The religion-health connection: Evidence, theory and future directions. Health Education and Behaviour, 25(6), 700-720.

Emmons, R. A. (1986). Personal strivings: An approach to personality and subjective wellbeing. Journal of Personality and Social Psychology, 51, 1058-1068.

Emmons, R. A. (1999). The psychology of ultimate concerns : Motivation and spirituality in personality. New York ; London: Guilford Press.

Emmons, R. A. (2005). Striving for the sacred: Personal goals, life meaning, and religion. Journal of Social Issues, 61(4), 731-745.

Emmons, R. A., \& McCullough, M. E. (2004). The psychology of gratitude. Oxford: Oxford University Press.

Emmons, R. A., \& Paloutzian, R. F. (2003). The psychology of religion. Annual Review of Psychology, 54, 377-402.

Endacott, G. B. (1964). A History of Hong Kong. Hong Kong: Oxford University Press.

Erikson, E. H. (1964). Insight and responsibility (1st ed.). New York: W. W. Norton.

Erikson, E. H. (1968). Identity, youth, and crisis (1st ed.). New York: W. W. Norton.

Estes, R. J. (2005). Social development in Hong Kong: The unfinished agenda. In R. J. Estes \& Hong Kong Council of Social Service. (Eds.), Social development in Hong Kong : the unfinished agenda. Oxford ; New York: Oxford University Press.

Ferraro, K. F., \& Albrecht-Jensen, C. M. (1991). Does religion influence adult health? Journal for the Scientific study of religion, 30(2), 193-202.

Field, A. P. (2005). Discovering statistics using SPSS (2nd ed.). London: SAGE Publications.

Fiese, B. H., \& Tomcho, T. J. (2001). Finding meaning in religious practices: The relation between religious holiday rituals and marital satisfaction. Journal of Family Psychology, 15, 597-609.

Finke, R. (2003). Spiritual capital: Definitions, applications, and new frontiers. Retrieved June 112006 from http://www.spiritualcapitalresearchprogram.com/pdf/Finke.pdf.

Fisher, A. T., \& Sonn, C. C. (2007). Sense of community and dynamics of inclusionexclusion by receiving communities. Australian Community Psychologist, 19(2), 2634.

Fisher, A. T., Sonn, C. C., \& Bishop, B. J. (2002). Psychological sense of community : Research, applications, and implications. New York: Kluwer Academic/Plenum Publishers.

Fiske, A. P., Kitayama, S., Markus, H. R., \& Nisbett, R. E. (1998). The cultural matrix of social psychology. In D. T. Gilbert, S. T. Fiske \& L. Gardner (Eds.), The handbook of social psychology (Vol. 2, pp. 915-981). Boston: McGraw-Hill

Fitzapatrick, R. (1996). Alternative approaches to the assessment of health-related quality of life. In A. Offer (Ed.), Pursuit of the Quality of Life. Oxford: Oxford University Press.

Flyvbjerg, B. (2001). Making social science matter: Why social inquiry fails and how it can succeed again. Cambridge: Cambridge University Press.

Foster-Fishman, P. G., \& Behrens, T. R. (2007). Systems change reborn: Rethinking our theories, methods, and efforts in human services reform and community-based change. American Journal of Community Psychology, 39, 191-196. 
Foster-Fishman, P. G., Nowell, B., \& Yang, H. (2007). Putting the system back into system change: A framework for understanding and changing organizational and community systems. American Journal of Community Psychology, 39, 197-215.

Frabricatore, A. N., Paul, J. H., Doris, M. R., \& Frank, H. G. (2004). Stress, religion, and mental health: Religious coping in mediating and moderating roles. The International Journal for the Psychology of Religion, 14, 91-108.

Fredrickson, B. L. (2001). The role of positive emotions in positive psychology: The broaden-and-build theory of positive emotions. American Psychologist, 56(3), 218226.

Fredrickson, B. L. (2008). Promoting positive affect. In M. Eid \& R. J. Larsen (Eds.), The science of subjective well-being. New York: Guilford Press.

Freire, P. (1972). Pedagogy of the oppressed. London: Panguin Books.

Friedman, M., \& Rholes, W. S. (2008). Religious fundamentalism and terror management. The International Journal for the Psychology of Religion, 18, 36-52.

Funder, D. C. (2002). Why study religion? Psychological Inquiry, 13(3), 213-214.

Gallimore, R., Goldenberg, C. N., \& Weisner, T. S. (1993). The social construction and subjective reality of activity settings: Implications for community psychology. American Journal of Community Psychology, 21(4), 539-559.

Geertz, C. (1973). The interpretation of Cultures. New York: Basic Books.

George, L. K., Ellison, C. G., \& Larson, D. B. (2002). Explaining the relations between religious involvement and health. Psychological Inquiry, 13(3), 190-200.

Giddens, A. (1979). Central problems in social theory : Action, structure, and contradiction in social analysis. London: Macmillan.

Giddens, A. (1984). The constitution of society: Outline of the theory of structuration. Berkeley: University of California Press.

Giddens, A., Appelbaum, R. P., \& Duneier, M. (2005). Introduction to sociology (5th ed. ed.). New York: W.W. Norton.

Glock, C. Y. (1962). On the study of religious commitment. Religious Education, 57, S98S110.

Goffman, E. (1959). The presentation of self in everyday life. Garden City, N.Y: Doubleday.

Gorsuch, R. L. (1984). Measurement: The boon and bane of investigating religion. American Psychologist, 39(3), 228-236.

Granovetter, M. (1973). The strength of weak ties. American Journal of Sociology, 78(6), 1360-1380.

Greenberg, A. (2000). The church and the revitalization of politics and community. Political Science Quarterly, 15(3), 377-394.

Greene, J. C. (2007). Mixed methods in social inquiry (1st ed.). San Francisco, CA: JosseyBass.

Greene, J. C., \& Caracelli, V. J. (2003). Making paradigmatic sense of mixed methods practice. In C. Teddlie \& A. Tashakkori (Eds.), Handbook of mixed methods in social \& behavioural research. Thousand Oaks, CA.: SAGE Publications.

Greenfield, P. M. (2000). Three approaches to the psychology of culture: Where do they come from? Where can they go? . Asian Journal of Social Psychology, 3, 223-240.

Haas, B. K. (1999). A multidisciplinary concept analysis of quality of life. Western Journal of Nursing Research, 21, 728-742.

Hackney, C. H., \& Sanders, G. S. (2003). Religiosity and mental health: A meta-analysis of recent studies. Journal for the Scientific study of religion, 42(1), 43-55. 
Hair, J. F. (2006). Multivariate data analysis (6th ed.). Upper Saddle River, N.J.: Pearson Prentice Hall.

Haralambos, M., \& Holborn, M. (1995). Sociology: Themes and perspectives (4th ed.). London: Collins Educational.

Hays, S. (1994). Structure and agency and the sticky problem of culture. Sociological Theory, 12(1), 57-72.

Helliwell, J. F. (2002). Globalization and well-being. Vancouver: UBC Press.

Helliwell, J. F., \& Putnam, R. D. (2004). The social context of well-being. Philosophical Transactions of the Royal Society B, 359(1449), 1435-1446.

Helminiak, D. A. (2008). Spirituality for our global community: beyond traditional religion to a world at peace. Lanham, Md.: Rowman \& Littlefield Publishers.

Herriot, P. (2007). Religious fundamentalism and social identity. London ; New York: Routledge.

Hill, J. (2000). A rationale for the integration of spirituality into community psychology. Journal of Community Psychology, 28, 139-149.

Hill, P. C. (2005). Measurement in the psychology of religion and spirituality. In R. F. Paloutzian \& C. L. Park (Eds.), Handbook of the psychology of religion and spirituality. New York: Guilford Press.

Hill, P. C., \& Hood, R. W. (Eds.). (1999). Measures of religiosity. Birmingham, Alabama: Religious Education Press.

Hill, P. C., \& Pargament, K. I. (2003). Advances in the conceptualization and measurement of religion and spirituality: Implication for Physical and mental health research. American Psychologist, 58(1), 64-74.

Hochschild, A. R. (1983). The managed heart : Commercialization of human feeling. Berkeley: University of California Press.

Holmes-Smith, P., Cunningham, E., \& Coote, L. (2006). Structural equation modelling: From the fundamentals to advanced topics. Melbourne: SREAMS, STATSLINE.

Hood, R. W. (1995). Handbook of religious experience. Birmingham, Ala.: Religious Education Press.

Hood, R. W., Hill, P. C., \& Williamson, W. P. (2005). The psychology of religious fundamentalism. New York: Guilford Press.

Hughes, R. (1976). Borrowed place, borrowed time : Hong Kong and its many faces (2nd rev. ed.). London: Deutsch.

Hunsberger, B. (1995). Religion and Prejudice: The role of religion fundamentalism, quest and right-wing. Journal of Social Issues, 51(2), 113-129.

Hunsberger, B., Alisat, S., Pancer, S. M., \& Pratt, M. (1996). Religious fundamentalism and religious doubts: Content, connections, and complexity of thinking. The International Journal for the Psychology of Religion, 6(3), 201-220.

Hunsberger, B., \& Jackson, L. M. (2005). Religion, meaning, and prejudice. Journal of Social Issues, 61(4), 807-826.

Jahoda, M. (1958). Current concepts of positive mental health. New York,: Basic Books.

James, W. (1902/ 1960). The varieties of religious experience (2nd ed.). New York: Longman Green.

Joiner, T. E., Hollar, D., \& Van Orden, K. (2006). On Buckeyes, Gators, Super Bowl Sunday, and the miracle on ice: "Pulling Together" is associated with lower suicide rates. Journal of Social and Clinical Psychology, 25(2), 179-195. 
Joseph, S., Linley, P. A., \& Maltby, J. (2006). Positive psychology, religion and spirituality. Mental Health, Religion and Culture, 9(3), 209-212.

Karoly, P. (1999). A goal systems-self-regulatory perspective on personality, psychopathology, and change. Review of General Psychology, 3, 264-291.

Kasser, T. (2000). Two versions of the American dream: Which goals and values make for a high quality of life? In E. Diener \& D. R. Rahtz (Eds.), Advances in quality of life theory and research. Dordrecht ; Boston: Kluwer Academic Publishers.

Kawachi, I., \& Berkman, L. F. (2000). Social epidemiology. New York ; Oxford: Oxford University Press.

Kelley, D. M. (1972). Why conservative churches are growing; A study in sociology of religion ([1st ed.). New York: Harper \& Row.

Kelly, J. G., Ryan, A. M., Altman, B. E., \& Stelzner, S. P. (2000). Understanding and changing social systems: an ecological view. In J. Rappaport, \& Seidman, E., (Ed.), The handbook of community psychology (pp. 495-522). New York: Kluwer Academic/ Plenum Publishers.

Kennedy, J. E. (1999). Religious guilt and fear, well-being, and fundamentalism. Unpublished Manuscript. Retrieved 2 February 2009 from http://jeksite.org/research/bv.htm.

Keyes, C. L. M. (1998). Social well-being. Social Psychology Quarterly, 61, 121-140.

Keyes, C. L. M. (2006). Subjective well-being in mental health and human development research worldwide: An introduction. Social Indicators Research, 77, 1-10.

Keyes, C. L. M., \& Lopez, S. J. (2002). Toward a science of mental health: Positive directions in diagnosis and treatment. In C. R. Snyder \& S. J. Lopez (Eds.), Handbook of positive psychology. Oxford England ; New York: Oxford University Press.

Keyes, C. L. M., Shmotkin, D., \& Ryff, C. D. (2002). Optimizing well-being: The empirical encounter of two traditions. Journal of Personality and Social Psychology, 82(6), 1007-1023.

Keyes, C. L. M., \& Waterman, M. B. (2003). Dimensions of well-being and mental health in adulthood. In B. M. H. (Ed.), Well-being : Positive development across the life course Mahwah, N.J.: Lawrence Erlbaum Associates.

King, L. A. (2008). Interventions for enhancing subjective well-being. In M. Eid \& R. J. Larsen (Eds.), The science of subjective well-being. New York: Guilford Press.

King, P. E., \& Furrow, J. L. (2004). Religion as a resource for positive youth development: Religion, social capital, and moral outcomes. Developmental Psychology, 40(5), 703713.

King, V. (2003). The influence of religion on fathers' relationships with their children. Journal of Marriage and Family, 65, 382-395.

Kitayama, S., \& Markus, H. R. (2000). The pursuit of happiness and the realization of sympathy: Cultural patterns of self, social relations, and well-being. In E. Diener \& E. M. Suh (Eds.), Culture and subjective well-being. Cambridge, Mass. ; London: MIT Press.

Kitayama, S., \& Park, H. (2007). Cultural shaping of self, emotion, and well-being: How does it work? Social and Personality Psychology Compass, 1(1), 202-222.

Kline, R. B. (2005). Principles and practice of structural equation modelling (2nd ed.). New York: Guilford Press.

Kloos, B., \& Moore, T. (2000a). Introduction to special issue on spirituality, religion and community psychology. Journal of Community Psychology, 28(2), 115-118. 
Kloos, B., \& Moore, T. (2000b). The prospect and purpose of locating community research and action in religious settings. Journal of Community Psychology, 28(2), 119-137.

Kluger, J. (2009). How faith can heal. TIME, 173, 31-45.

Knorr-Cetina, K. (1981). The micro-sociological challenge of macro-sociology: Towards a reconstruction of social theory and methodology. In K. Knorr-Centina \& A. V. Cicourel (Eds.), Advances in social theory and methodology : toward an integration of micro- and macro-sociologies Boston: Routledge \& Kegan Paul.

Koenig, H. G. (1998). Handbook of religion and mental health. San Diego: Academic Press.

Koenig, H. G. (2005). Faith and mental health : religious resources for healing. Philadelphia: Templeton Foundation Press.

Koenig, H. G., McCullough, M. E., \& Larson, D. B. (2000). Handbook of religion and health. New York ; Oxford: Oxford University Press.

Kral, M. J. (2007). Psychology and anthropology: Intersubjectivity and epistemology in an interpretive cultural science. Journal of Theoretical and Philosophical Psychology, 27(2), 257-275.

Kramer, R. M. (1999). Trust and distrust in organizations: Emerging perspectives, enduring questions. Annual Review of Psychology, 50, 569-598.

Kung, L. Y. (2004). Politics and religions in Hong Kong after 1997: Whether tension or equilibrium is needed. Religion, State and Society, 32(1), 21-36.

Kwok, N. W. (1997). Christian churches in Hong Kong under colonial rule. Tripod, 98, $28-42$.

Kwok, W. L. (2001). Social participation of the Hong Kong Protestant church in the early eighties: An analysis of her conviction. CGST Journal, 30(1), 13-52.

Kwok, W. L. (2002). The social participation of Hong Kong young evangelicals in the seventies. CGST Journal, 32(1), 145-190.

Kwong, C. w. (2000). Hong Kong's religions in transition : Confucianism, Taoism, Buddhism, and Christianity, and the restructuring of their public roles during Hong Kong's incorporation into mainland China (1984-1998) (1st ed.). Waco, Tex.: Tao Foundations.

Kwong, C. W. (2001). The social roles of Hong Kong churches after the return to Chinese sovereignty in 1997. Journal of the History of Christianity in Modern China, 4, 56-70.

Lam, C. L. K., Tse, E., Y. Y., \& Gandek, B. (2005). Is the standard SF-12 Health Survey valid and equivalent for a Chinese population? Quality Life Research, 14, 539-547.

Lam, P. Y. (2002). As the flocks gather: How religion affects voluntary association participation. Journal for the Scientific study of religion 41(3), 405-422.

Lam, W. F., \& Perry, J. L. (2000). The role of the nonprofit sector in Hong Kong's development. Voluntas: International Journal of Voluntary and Non-profit Organizations, 11(4), 355-373.

Lam, W. M. (2007). Political Context. In W.-m. Lam (Ed.), Contemporary Hong Kong politics : governance in the post-1997 era. Hong Kong: Hong Kong University Press.

Lam, W. M., \& Tong, L. K. (2007). Civil society and NGOs. In W.-m. Lam (Ed.), Contemporary Hong Kong politics: Governance in the post-1997 era. Hong Kong: Hong Kong University Press.

Langer, E. (2002). Well-being: Mindfulness versus positive evaluation. In C. R. Snyder \& S. J. Lopez (Eds.), Handbook of positive psychology. Oxford: Oxford University Press.

Larson, J. S. (1996). The world health organization's definition of health: Social versus spiritual health. Social Indicators Research, 38, 181-192. 
Lau, S. K., \& Kuan, H. C. (1988). The ethos of the Hong Kong Chinese. Hong Kong: The Chinese University Press.

Lavric, M., \& Flere, S. (2008). The role of culture in the relationship between religiosity and psychological well-being. Journal of Religion and Health, 47, 164-175.

Laythe, B., Finkel, D., \& Kirkpatrick, L. A. (2001). Predicting prejudice from religious fundamentalism and right-wing authoritarianism: A multiple-regression approach. Journal for the Scientific Study of Religion, 40(1), 1-10.

Lazar, A. (2004). Cultural influences on religious experience and motivation. Review of Religious Research, 46(1), 64-71.

Lenski, G. (1963). The religious factor: A sociological study of religion's impact on politics, economics, and family Life. (rev. ed.). Garden City, NJ: Anchor.

Leung, K., \& Bond, M. H. (2004). Social axioms: A model for social beliefs in multi-cultural perspective. Advances in Experimental Social Psychology, 36, 119-197

Levin, J. S. (1994). Religion in aging and health: Theoretical foundations and methodological frontiers. Thousand Oaks: Sage Publications.

Levin, J. S. (2001). God, faith, and health: Exploring the spirituality-healing connection. New York: John Wiley \& Sons, Inc. .

Levin, J. S., \& Chatters, L. M. (1998). Research on religion and mental health: An overview of empirical findings and theoretical issues. In H. G. Koenig (Ed.), Handbook of Religion and Mental Health. San Diego, CA. : Academic Press.

Lewin, K. (1951). Field theory in social science. New York: Harper and Row.

Li, Y., Pickles, A., \& Savage, M. (2005). Social capital and social trust in Britain. European Sociological Review, 21(2), 109-123.

Lin, N. (2001). Social capital : A theory of social structure and action. Cambridge ; New York: Cambridge University Press.

Lincoln, Y. S., \& Guba, E. G. (1985). Naturalistic inquiry. Beverly Hills, CA.: Sage Publications.

Liu, Y. (1941). Xianggang Jidu jiao hui shi. Xianggang: Xianggang Jidu jiao lian hui.

Lo, H. l. (1963). Role of Hong Kong in the cultural interchange between East \& West. Tokyo: Centre for East Asian Cultural Studies.

Lofland, J. (2006). Analyzing social settings : A guide to qualitative observation and analysis (4th ed.). Belmont, CA.: Wadsworth Pub. Co.

Loveland, M., T. , Sikkink, D., Myers, D. J., \& Radcliff, B. (2005). Private prayer and civic Involvement. Journal for the Scientific study of religion, 44(1), 1-14.

Lui, T. K. (1997). "Check, please!" : A Sociologist's Notes on Hong Kong. Hong Kong: Xian Ren Hang Publisher (in Chinese).

Luk, B. H. K. (1989). Religion and custom. In T. L. Tsim \& B. H. K. Luk (Eds.), The Other Hong Kong Report 1989. Hong Kong: CUHK Press.

Luke, D. A., Rappaport, J., \& Seidman, E. (1991). Setting phenotypes in a mutual help organization: Expanding behaviour setting theory. American Journal of Community Psychology, 19(1), 147-167.

Lyubomirsky, S., Sheldon, K. M., \& Schkade, D. (2005). Pursuing happiness: The architecture of sustainable change. Review of General Psychology, 9(2), 111-131.

Ma, N. (2007). Political development in Hong Kong: State, political society, and civil society. Hong Kong Hong Kong University Press.

MacKinnon, D. P., Fairchild, A. J., \& Fritz, M. S. (2007). Mediation analysis. Annual Review of Psychology, 58, 593-614. 
Mahoney, A., Pargament, K. I., Tarakeshwar, N., \& Swank, S. B. (2001). Religion in the home in the 1980s and 1990s: A meta-analytic review and conceptual analysis of links between religion, marriage, and parenting. The Journal of Family Psychology 15, 559-596.

Malloch, T. R. (2003). Social, human and spiritual capital in economic development. Retrieved June 112006 from http://www.spiritualcapitalresearchprogram.com/pdf/Berger.pdf.

Manderson, L. (2005). Rethinking wellbeing. Perth, W.A.: API Network.

Mankowski, E. S., \& Rappaport, J. (2000). Narrative concepts and analysis in spiritually based communities. Journal of Community Psychology, 28(5), 479-493.

Markus, H., \& Kitayama, S. (1994). The cultural construction of self and emotion: Implications for social behavior. In S. Kitayama \& H. Markus (Eds.), Emotion and culture : Empirical studies of mutual influence (1st ed.). Washington, DC: American Psychological Association.

Markus, H. R., \& Kitayama, S. (1991). Culture and the self: Implications for Cognition, Emotion, and Motivation. Psychological Review, 98(2), 224-253.

Marmot, M. G., \& Wilkinson, R. G. (Eds.). (1999). Social Determinants of Health. New York: Oxford University Press.

Martin, J., \& Frost, P. (1996). The organizational culture war games: A struggle for intellectual dominance. In C. Hardy, W. R. Nord \& S. Clegg (Eds.), Handbook of organization studies (pp. xxix, 730). London: Sage.

Maton, K. (2008). Habitus. In M. Grenfell (Ed.), Pierre Bourdieu : key concepts. Stocksfield England: Acumen Pub.

Maton, K. I. (1993). A bridge between cultures: Linked ethnographic empirical methodology for culture anchored research. American Journal of Community Psychology, 21(6), 747-773.

Maton, K. I. (2000). Narrative concepts, spiritually based communities: Strengths and future development. Journal of Community Psychology, 28(5), 529-533.

Maton, K. I. (2001). Spirituality, religion, and community psychology: Historical perspective, positive potential, and challenge. Journal of the Scientific Study of Relgion, 29(5), 605-613.

Maton, K. I. (2008). Empowering community settings: Agents of individual development, community betterment, and positive social change. American Journal of Community Psychology, 41, 4-21.

McCrohan, B., \& Bernt, F. M. (2004). Service and worship as modes of religious expression among Catholic college students. The International Journal for the Psychology of Religion, 14(4), 273-284.

McCullough, M. E. (2000). Forgiveness as a human strength: Theory, measurement, and links to well-being. Journal of Social and Clinical Psychology, 19, 43-55.

McCullough, M. E., Pargament, K. I., \& Thoresen, C. E. (2000). Forgiveness : Theory, research, and practice. New York: Guilford Press.

McCutcheon, R. T. (1999). The insider/outsider problem in the study of religion: a reader. London ; New York: Cassell.

McGregor, I., \& Little, B. R. (1998). Personal projects, happiness, and meaning: On doing well and being yourself. Journal of Personality and Social Psychology, 74, 494-512.

McGuire, M. B. (2002). Religion: the social context (5th ed.). Belmont, CA. ; London: Wadsworth. 
McKenzie, K., \& Harpham, T. (2006a). Social capital and mental health. Philadelphia, PA: Jessica Kingsley Publishers.

McKenzie, K., \& Harpham, T. (2006b). Meanings and uses of social capital in the mental health field. In K. McKenzie \& T. Harpham (Eds.), Social capital and mental health. Philadelphia, PA: Jessica Kingsley Publishers.

Mckenzie, K., Whitley, R., \& Weich, S. (2002). Social capital and mental health. British Journal of Psychiatry, 181, 280-283.

McMillan, D. W., \& Chavis, D. M. (1986). Sense of community: A definition and theory. Journal of Community Psychology, 14, 6-23.

McPherson, M., Smith-Lovin, L., \& Cook, J. M. (2001). Birds of a feather: Homophily in social network. Annual Review of Sociology, 27, 415-444.

Mead, G. H. (1934). Mind, self \& society from the standpoint of a social behaviourist. Chicago, Ill.: The University of Chicago press.

Meyers, L. S., Gamst, G., \& Guarino, A. J. (2006). Applied multivariate research: Design and interpretation. Thousand Oaks: SAGE Publications.

Michalos, A. C. (2004). Social indicators research and health-related quality of life. Social Indicators Research, 65(1), 27-72.

Miers, R., \& Fisher, A. T. (2002). Being church and community: Psychological sense of community in a local parish. In A. T. Fisher, C. C. Sonn \& B. J. Bishop (Eds.), Psychological sense of community: Research, applications, and implications. New York: Kluwer Academic/Plenum Publishers.

Miles, M. B., \& Huberman, A. M. (1994). Qualitative data analysis : An expanded sourcebook (2nd ed. ed.). Thousand Oaks: Sage Publications.

Miller, J. (1997). The interdependence of interpretive ethnographic and quantitative psychological methodologies in cultural psychology. Ethos, 25(2), 164-176.

Miller, P. J., Hengst, J. A., \& Wang, S. H. (2003). Ethnographic methods: Applications from developmental cultural psychology. In P. M. Camic, J. E. Rhodes \& L. Yardley (Eds.), Qualitative Research in Psychology: Expanding perspectives in methodology and design. Washington DC: American Psychological Association.

Miller, W. R., \& Thoresen, C. E. (2003). Spirituality, religion, and health: An emerging research field. American Psychologist, 58(1), 24-35.

Moore, T., Kloos, B., \& Rasmussen, R. (2001). A reunion of ideas: Complementary inquiry and collaborative interventions of spirituality, religion, and psychology. Journal of Community Psychology, 29(5), 487-495.

Morgan, P. D., Tyler, I. D., \& Fogel, J. (2008). Fatalism revisited. Seminars in Oncology Nursing, 24(4), 237-245.

Morse, J. M. (1991). Approaches to qualitative-quantitative methodological triangulation Nursing Research, 40, 120-123.

Murray, K., \& Ciarrocchi, J. W. (2007). The dark side of religion, spirituality and the moral emotions: Shame, guilt, and negative religiosity as markers for life dissatisfaction. Journal of Pastoral Counseling, 42, 22-41.

Murray, M. (2000). Levels of narrative analysis in health psychology. Journal of Health Psychology, 5(3), 337-347.

Murray, M. (2003). Narrative psychology and narrative analysis. In P. M. Camic, Rhodes, J. E., \& Yardley, L. (Ed.), Qualitative Research in Psychology: Expanding perspectives in methodology and design. Washington DC: American Psychological Association. 
Myers, D. G. (1999). Close relationships and quality of life. In D. Kahneman, E. Diener \& N. Schwarz (Eds.), Well-being: The foundations of hedonic psychology. New York: Russell Sage Foundation.

Myers, D. G. (2007). Building on the "third pillar": Positive communities. In C. R. Snyder \& S. J. Lopez (Eds.), Positive psychology: the scientific and practical explorations of human strengths (pp. 490). Thousand Oaks, CA.: SAGE Publications.

Myers, D. G. (2008). Religion and human flourishing. In M. Eid \& R. J. Larsen (Eds.), The science of subjective well-being. New York: Guilford Press.

Myers, D. G., \& Diener, E. (1995). Who is Happy? Psychological Science, 6(1), 10-19.

Nelson, G. B., \& Prilleltensky, I. (2005). Community Psychology: In pursuit of Liberation and Wellbeing. New York: Palgrave Macmillan.

Neuman, W. L. (2006). Social research methods: Qualitative and quantitative approaches (6th ed. ed.). Boston: Pearson/Allyn and Bacon.

Newton, K. (2001). Trust, social capital, civil society, and democracy. International Political Science Review, 22(2), 201-214.

Newton, K. (2004). Social trust: Individual and cross-national approaches. Portuguese Journal of Social Science, 3(1), 15-35.

Ng Lun, N. H. (1989). History. In T. L. Tsim \& B. H. K. Luk (Eds.), The Other Hong Kong Report 1989. Hong Kong: CUHK Press.

Nicholl, T. (2006). Vygotsky : An introduction to culturally-mediated identity Retrieved 22 May 2006, from www.massey.ac.nz/ alock/virtual/trishvyg.htm

Niebuhr, H. R. (1929). The social sources of denominationalism. Cleveland, New York: World Pub. Co.

Nock, S. L. (1998). Marriage in men's lives. New York: Oxford University Press.

Noren, L. E. (1963). Urban church growth in Hong Kong 1958-1962 : Third Hong Kong study. Kowloon, Hong Kong: n. p.

O' Donnell, C. R., Tharp, R. G., \& Wilson, K. (1993). Activity settings as the unit of analysis: A theoretical basis for community intervention and development. American Journal of Community Psychology, 21(4), 501-520.

O' Neill, P. (2005). The ethics of problem definition. Canadian Psychology, 46(1), 13-20.

Obst, P., \& Tham, N. (2009). Helping the soul: The relationship between connectivity and well-being within a church community. Journal of Community Psychology, 37(3), 342-361.

Obst, P., Zinkiewicz, L., \& Smith, S. G. (2002). Sense of community in science fiction fandom, Part 1: Understanding sense of community in an international community of Interest. Journal of Community Psychology, 30(1), 87-103.

Oishi, S. (2000). Goals as cornerstones of subjective well-being: Linking individuals and cultures. In E. Diener \& E. M. Suh (Eds.), Culture and subjective well-being. Cambridge, Mass. ; London: MIT Press.

Otto, R. (1917). The Idea of the Holy. London: Oxford University Press.

Paloutzian, R. F., \& Kirkpatrick, L. A. (1995). Introduction: The scope of religious influences on personal and societal well-being. Journal of Social Issues, 51(2), 1-11.

Paloutzian, R. F., \& Park, C. L. (2005). Integrative Themes in the current science of the psychology of religion. In R. F. Paloutzian \& C. L. Park (Eds.), Handbook of the psychology of religion and spirituality (pp. 3-20). New York: Guilford Press.

Pals, D. L. (2006). Eight theories of religion. New York: Oxford University Press. 
Pargament, K. I. (1999). The psychology of religion and spirituality? Yes and no. The International Journal for the Psychology of Religion, 9(1), 3-16.

Pargament, K. I. (2002a). The bitter and sweet: An evaluation of the costs and benefits of religiousness. Psychological Inquiry, 13(3), 168-181.

Pargament, K. I. (2002b). Is religion nothing but...? Explaining religion versus explaining religion away. Psychological Inquiry, 13(3), 239-244.

Pargament, K. I., Magyar-Russell, G. M., \& Murray-Swank, N. A. (2005). The sacred and the search for significance: Religion as a unique process. Journal of Social Issues, 61(4), 665-687.

Pargament, K. I., Magyar, G. M., \& Benore, E. M., Annette. (2005). Sacrilege: A study of sacred loss and desecration and their implications for health and well-being in a community sample. Journal for the Scientific study of religion, 44(1), 59-78.

Pargament, K. I., \& Mahoney, A. (2002). Spirituality: Discovering and conserving the sacred. In C. R. Snyder \& S. J. Lopez (Eds.), Handbook of positive psychology. Washington, DC: APA Press.

Pargament, K. I., \& Mahoney, A. (2005). Sacred matters: Sanctification as a vital topic for the psychology of religion. The International Journal for the Psychology of Religion, 15(3), 179-198.

Pargament, K. I., \& Maton, K. I. (2000). Religion in American life: A community psychology perspective. In J. Rappaport, \& Seidman, E., (Ed.), The handbook of community psychology (pp. 495-522). New York: Kluwer Academic/ Plenum Publishers.

Park, C. L., \& Paloutzian, R. F. (2005). One step toward integration and an expansive future. In R. F. Paloutzian \& C. L. Park (Eds.), Handbook of the psychology of religion and spirituality (pp. 550-564). New York: Guilford Press.

Park, J. Z., \& Smith, C. (2000). "To whom much has been given..." Religious capital and community voluntarism among churchgoing Protestants. Journal for the Scientific Study of religion, 39, 272-286.

Peacock, J. R., \& Poloma, M. M. (1998). Religiosity and life satisfaction across the life course. Social Indicators Research, 48, 321-345.

Pearce, L. D., \& Axinn, W. G. (1998). The impact of family religious life on the quality of mother-child relations American Sociological Review, 63, 810-828.

Perkins, D. D., Florin, P., Rich, R., Wandersman, A., \& Chavis, D. M. (1990). Participation and the social and physical environment of residential blocks: Crime and community context. American Journal of Community Psychology, 18, 83-115.

Peterson, C., \& Seligman, M. E. P. (2004). Character strengths and virtues: A handbook and classification. New York: Oxford University Press.

Phillips, D. (2006). Quality of life: Concept, policy and practice. London ; New York: Routledge.

Pichon, I., Boccato, G., \& Saroglou, V. (2007). Nonconscious influences of religion on prosociality: A priming study. European Journal of Social Psychology, 37, 1032-1045.

Pienaar, J. M., Beukes, R. B. I., \& Esterhuyse, K. G. F. (2006). Te relationship between conservatism and psychological well-being in adolescents. South African Journal of Psychology, 36(2), 391-406.

Poloma, M. M. (1995). The Sociological Context of Religious Experience. In R. W. Hood (Ed.), Handbook of religious experience (pp. 661 p.). Birmingham, Ala.: Religious Education Press. 
Poloma, M. M., \& Pendleton, B. R. (1991). The effects of prayer and prayer experiences on measures of general well-being Journal of Psychology and Theology, 19, 71-83.

Pratt, J. B. (1924). The religious consciousness. New York: Macmillan.

Pratt, M. G., \& Doucet, L. (2000). Ambivalent feelings in organizational relationships. In S. Fineman (Ed.), Emotion in organizations (2nd ed., pp. viii, 289 p.). London: SAGE.

Pretty, G. M. H. (2002). Young people's development of the community-minded self: Considering community identity, community attachment and sense of community. In A. T. Fisher, C. C. Sonn \& B. J. Bishop (Eds.), Psychological sense of community : Research, applications, and implications. New York: Kluwer Academic/Plenum Publishers.

Prezza, M., Amici, M., Roberti, T., \& Tedeschi, G. (2001). Sense of community referred to the whole town: Its relations with neighbouring, loneliness, life satisfaction, and area of residence. Journal of Community Psychology, 28(29-52).

Prezza, M., \& Costantini, S. (1998). Sense of community and life satisfaction: Investigation in three different territorial contexts. Journal of Community \& Applied Social Psychology, 8(3), 181-194.

Prilleltensky, I., \& Prilleltensky, O. (2006). Promoting well-being : Linking personal, organizational, and community change. Hoboken, N.J.: John Wiley.

Primavera, J., \& Brodsky, A. E. (2004). Introduction to the special issue on the process of community research and action. American Journal of Community Psychology, 33(3), 177-179.

Proescholdbell, R. J., Roosa, M. W., \& Nemeroff, C. J. (2006). Component measures of psychological sense of community among gay men. Journal of Community Psychology, 34(1), 9-24.

Putnam, R. D. (2000). Bowling alone: The collapse and revival of American community. New York ; London: Simon \& Schuster.

Raphael, D., Renwick, R., Brown, I., Steinmetz, B., Sehdev, H., \& Phillips, S. (2001). Making the links between community structure and individual well-being: Community quality of life in Riverdale, Toronto, Canada. Health and Place, 7(179196).

Rapkin, B. D., \& Mulvey, E. P. (1990). Criteria of excellence III. Methods of studying community psychology: Towards excellence in quantitative community research. In P. Tolan, Keys, C., Chertok, F., \& Jason, L. (Ed.), Research Community Psychology. Washington, DC.: American Psychological Association.

Rapley, M. (2003). Quality of life research : a critical introduction. London: SAGE.

Rate, C. R., Clarke, J. A., Lindsay, D. R., \& Sternberg, R. J. (2007). Implicit theories of courage. The Journal of Positive Psychology, 2(2), 80 - 98.

Ratner, C. (1999). Three approaches to cultural psychology: A critique. Cultural Dynamics, $11,7-31$.

Ratner, C. (2006). Cultural psychology : A perspective on psychological functioning and social reform. Mahwah, N.J.: Lawrence Erlbaum Associates.

Regnerus, M., Smith, C. T., \& Firitsch, M. (2003). Religion in the lives of American adolescents: A review of the literature. Chapel Hill, NC: National Study of Youth and Religion.

Riger, S. (2001). Transforming community psychology. American Journal of Community Psychology, 29(1), 69-81. 
Roberts, B. (2006). Micro social theory. Houndmills, Basingstoke, Hampshire: Palgrave Macmillan.

Rogoff, B. (1990). Apprenticeship in thinking: cognitive development in social context. New York: Oxford University Press.

Rogoff, B. (2003). The cultural nature of human development. New York: Oxford University Press.

Roozen, D. A., McKinney, W., \& Carroll, J. W. (1984). Varieties of religious presence. New York: Pilgrim Press.

Rotter, J. B. (1967). A new scale for the measurement of interpersonal trust. Journal of Personality, 35(4), 615-665.

Rubin, J. (2008). Melancholy. In J. Corrigan (Ed.), The Oxford Handbook of Religion and Emotion. New York: Oxford University Press.

Ryan, R. M., \& Deci, E. L. (2001). On happiness and human potentials: A review of research on hedonic and eudaimonic well-being. Annual Review of Psychology, 52, 141-166.

Ryff, C. D. (1989). Happiness is everything, or is it? Explorations on the meaning of psychological well-being. Journal of Personality and Social Psychology, 57, 10691081.

Ryff, C. D., \& Keyes, C. L. M. (1995). The structure of psychological well-being revisited. Journal of Personality and Social Psychology, 69(4), 719-727.

Sachs, J. (2003). Validation of the satisfaction with life scale in a sample of Hong Kong university students. Psychologia, 46, 225-234.

Salsman, J. M., Brown, T. I., Brechting, E. H., \& Carlson, C. R. (2005). The link between religion and spirituality and psychological adjustment: The mediating role of optimism and social support. Personality and Social Psychology Bulletin, 31, 522-535.

Sampson, R. J. (1996). The community. In J. Q. Wilson, J. Petersilia \& Center for SelfGovernance. (Eds.), Crime. San Francisco, CA.: ICS Press, Institute for Contemporary Studies.

Sarason, S. B. (1974). The psychological sense of community: Prospects for a community psychology (1st ed.). San Francisco: Jossey-Bass.

Saroglou, V. (2002c). Beyond dogmatism: The need for closure as related to religion. Mental Health, Religion and Culture, 5(2), 183-194.

Saroglou, V. (2006). Religion's role in prosocial behavior: Myth or reality? Psychology of Religion Newsletter: American Psychological Association Division 36, 31(2), 1-8.

Schalock, R. L. (2000). Three decades of quality of life. In M. L. Wehmeyer \& J. R. Patton (Eds.), Mental retardation in the 21st century. Austin, TX: Pro-Ed.

Schalock, R. L., \& Felce, D. (2004). Quality of life and subjective well-being: Conceptual and measurement issues. In E. Emerson, C. Haton, T. Thompson \& T. R. Parmenter (Eds.), The international handbook of applied research in intellectual disabilities. .

Scheier, M. F., \& Carver, C. S. (1985). Optimism, coping, and health: Assessment and implications of generalized outcome expectancies. Health Psychology, 4(219-247).

Schumacker, R. E. (2006). Conducting specification searches with AMOS. Structural Equation Modelling, 13(1), 118-129.

Schumacker, R. E., \& Lomax, R. G. (2004). A beginner's guide to structural equation modeling (2nd ed.). Mahwah, N.J.: Lawrence Erlbaum Associates.

Schwadel, P. (2005). Individual, congregational, and denominational effects on church members' civic participation. Journal for the Scientific study of religion, 44(2), 159171. 
Schwartz, T. (1981). The acquisition of culture. Ethos, 9(1), 4-17.

Schwartzman, H. B. (1993). Ethnography in organizations. Newbury Park, CA.: Sage Publications.

Scott, W. R. (2003). Organizations: Rational, natural, and open systems (5th ed.). Upper Saddle River, N.J.: Prentice Hall.

Scott, W. R., \& Davis, G. F. (2007). Organizations and organizing: Rational, natural, and open systems perspectives ([6th ed.). Upper Saddle River, N.J.: Pearson Education, Inc.

Seeman, T. E., Dubin, L. F., \& Seeman, M. (2003). Religiosity/ spirituality and health: A critical review of the evidence for biological pathways. American Psychologist, 58(1), 53-63.

Segal, R. A. (1983/1999). In defense of reductionism. In R. T. McCutcheon (Ed.), The insider/outsider problem in the study of religion : a reader. London ; New York: Cassell.

Seligman, M. E. P. (1991). Learned optimism. New York: Knopf.

Seligman, M. E. P., \& Csikszentmihalyi, M. (2000). Positive psychology: An introduction. American Psychologist, 55(1), 5-14.

Sethi, S., \& Seligman, M. E. P. (1993). Optimism and fundamentalism. Psychological Science, 4(4), 256-259.

Shaffer, B. A., \& Hastings, B. M. (2007). Authoritarianism and religious identification: Response to threats on religious beliefs. Mental Health, Religion and Culture, 10(2), 151-158.

Shapiro, A., \& Keyes, C. L. M. (2008). Marital status and social well-being: Are the married always better off? Social Indicators Research, 88, 329-346.

Shariff, A. F., \& Norenzayan, A. (2007). God is watching you: Priming God concepts increases prosocial behavior in an anonymous economic game. Psychological Science, 18(9), 803-809.

Shinn, M., \& Yoshikawa, H. (2008). Toward positive youth development transforming schools and community programs. Oxford ; New York: Oxford University Press.

Silberman, I. (2005). Religion as a meaning system: Implications for the new millennium. Journal of Social Issues, 61(4), 641-663.

Silberman, I., Higgins, E. T., \& Dweck, C. S. (2005). Religion and world change: Violence and terrorism versus peace. Journal of Social Issues, 61(4), 761-784.

Silverman, D. (2001). Interpreting qualitative data: methods for analysing talk, text, and interaction (2nd ed.). London ; Thousand Oaks, CA.: Sage Publications.

Silverman, D., \& Seale, C. (2005). Doing qualitative research: A practical handbook (2nd ed.). London: SAGE.

Singelis, T. M., Hubbard, C., Her, P., \& An, S. (2003). Convergent validation of the social axioms survey. Personality and Individual Differences, 34, 269-282.

Sirgy, M. J., Michalos, A. C., Ferriss, A. L., Easterlin, R. A., Patrick, D., \& Pavot, W. (2006). The quality-of-life (QOL) research movement: Past, present, and future. Social Indicators Research, 76, 343-466.

Sirgy, M. J., \& Rahtz, D. (2006). A measure and method to assess subjective community quality of life. In M. J. Sirgy, D. Rahtz \& D. Swain (Eds.), Community quality of life indicators: Best Cases II (pp. 61-74). Netherlands: Springer.

Smart, N. (1996). Dimensions of the sacred: An anatomy of the world's beliefs. Berkeley: University of California Press. 
Smart, N. (1998). The world's religions (2nd ed.). Englewood Cliffs, N.J.: Prentice Hall.

Smidt, C. E. (2003). Religion as social capital: Producing the common good. Waco, TX: Baylor University Press.

Smith, J. A., Harré, R., \& Langenhove, L. V. (1995). Rethinking psychology. London: Saga Publications.

Smith, T. W. (1990). Classifying Protestant denominations. Review of Religious Research, 31(3), 225-245.

Smith, W. C. (1962/ 1991). The meaning and end of religion. Minneapolis: Fortress Press.

Snyder, C. R. (1994). The psychology of hope: You can get there from here. New York: Free Press.

Snyder, C. R. (2000). Handbook of hope: Theory, measures, and applications. Burlington: Elsevier.

Snyder, C. R., \& Lopez, S. J. (2002). Handbook of positive psychology. Oxford England ; New York: Oxford University Press.

Snyder, C. R., \& Lopez, S. J. (2007). Positive psychology: The scientific and practical explorations of human strengths. Thousand Oaks, CA.: SAGE Publications.

Sonn, C. C. (2002). Immigrant adaptation: Understanding the process through sense of community. In A. T. Fisher, C. C. Sonn \& B. J. Bishop (Eds.), Psychological sense of community : Research, applications, and implications. New York: Kluwer Academic/Plenum Publishers.

Sonn, C. C., \& Fisher, A. T. (1996). Psychological sense of community in a politically constructed group. Journal of Community Psychology, 24(4), 417-430.

Stake, R. E. (1995). The art of case study research. Thousand Oaks, CA.: Sage Publications.

Stake, R. E. (2006). Multiple case study analysis. New York: The Guilford Press.

Sternberg, R. J. (1990). Wisdom : Its nature, origins, and development. Cambridge ; New York: Cambridge University Press.

Sternberg, R. J. (1998). A balance theory of wisdom. Review of General Psychology, 2, 347365.

Suh, E. (2000). Self, the hyphen between culture and subjective well-being. In E. Diener \& E. M. Suh (Eds.), Culture and subjective well-being. Cambridge, Mass. ; London: MIT Press.

Sullivan, J. L., \& Transue, J. E. (1999). The psychological underpinnings of democracy: A selective review of research on political tolerance, interpersonal trust, and social capital. Annual Review of Psychology, 50, 625-650.

Sullivan, K. T. (2001). Understanding the relationship between religiosity and marriage: An investigation of the immediate and longitudinal effects of religiosity on newlywed couples. Journal of Family Psychology, 15, 610-626.

Super, C., \& Harkness, S. (1986). The developmental niche: A conceptualization at the interface of child anc culture. International Journal of Behavior Development, 9, 1-25.

Szaflarski, M. R., P. N. , Leonard, A. C., Mrus, J. M., Peterman, A. H., \& Tsevat, J. (2005). Modelling the effect of spirituality/ religion on patients' perceptions of living with HIV/ AIDS. Paper presented at the Annual Meeting of the American Sociological Association in Philadelphia, PA, USA.

Tabachnick, B. G., \& Fidell, L. S. (2007). Using multivariate statistics (5th ed.). Boston: Pearson/Allyn \& Bacon.

Tashakkori, A., \& Teddlie, C. (1998). Mixed methodology: combining qualitative and quantitative approaches. Thousand Oaks, CA.: Sage. 
Teddlie, C., \& Tashakkori, A. (2009). Foundations of mixed methods research: Integrating quantitative and qualitative techniques in the social and behavioural sciences. Thousand Oaks: Sage Publications.

Tedlock, B. (2000). Ethnography and ethnographic representation. In N. K. Denzin \& Y. S. Lincoln (Eds.), Handbook of qualitative research (2nd ed.). Thousand Oaks, CA.: Sage Publications.

Thoits, P. A., \& Hewitt, L. N. (2001). Volunteer work and well-being. Journal of Health and Social Behavior 42, 115-131.

Thomas, S. M. (2004). Building communities of character: Foreign aid policy and faith based organizations. SAIS Review, 24(2), 133-148.

Toro, P. A. (2005). Community psychology: Where do we go from here? American Journal of Community Psychology, 35, 9-16.

Triandis, H. C. (1995). Individualism and collectivism. Boulder: Westview Press.

Trickett, E. J. (1996). A future for community psychology: The contexts of diversity and the diversity of contexts. American Journal of Community Psychology, 24(2), 209-234.

Tse, T., K. C. (Ed.). (2002). Our place, our time: A new introduction to Hong Kong society. Hong Kong Oxford Unviersity Press (in Chinese).

Underwood, L. G. (2006). Ordinary spiritual experience: Qualitative research, interpretive guidelines, and population distribution for the daily spiritual experience scale. Archiv for the Psychology of Religion, 28, 181-218.

Underwood, L. G., \& Teresi, J. (2002). The daily spiritual experience scale: Development, theoretical description, reliability, exploratory factor analysis, and preliminary construct validity using health related data. . Annals of Behavioural Medicine, 24(1), 22-33.

Van der Veer, R. (2001). The idea of units of analysis: Vygotsky's contribution. In S. Chaiklin (Ed.), The theory and practice of cultural-historical psychology. Oxford: Aarhus University Press.

Veenhoven, R. (2008). Sociological Theories of Subjective Well-Being. In M. Eid \& R. J. Larsen (Eds.), The science of subjective well-being. New York: Guilford Press.

Verter, B. (2003). Spiritual capital: Theorizing religion with Bourdieu against Bourdieu. Sociological Theory, 21(2), 150-174.

VicHealth (2009a). Mental health and wellbeing: Everybody's business. VicHealth Letter, 34, 2.

VicHealth (2009b). THE MELBOURNE CHARTER for promoting mental health and preventing mental and behavioural disorder. VicHealth Letter, 34, 14-15.

Vilchinsky, N. K., S. (2005). How are religious belief and behavior good for you? An investigation of mediators relating religion to mental health in a sample of Israeli Jewish students Journal for the Scientific study of religion, 44(4), 459-471.

Vygotsky, L. S. (1978). Mind in society : The development of higher psychological processes. Cambridge: Harvard University Press.

Wald, K. D., Silverman, A. L., \& Fridy, K. S. (2005). Making sense of religion in political life. Annual Review of Political Science 8, 121-143.

Ware, J. E., Kosiinski, M., \& Keller, S. D. (1996). A 12-item short-form health survey. Construction of scales and preliminary tests of reliability and validity. Medical Care, 34, 220-233. 
Ware, J. E., Kosinski, M., Turner-Bowker, D. M., \& Gandeck, B. (2002). User's manual for the S-12v2 Health Survey: (with a supplement documenting SF-12 Health Survey). Lincoln: QualityMetric.

Waterman, A. S. (1993). Two conceptions of happiness: Contrasts of personal expressiveness (eudaimonia) and hedonic enjoyment. Journal of Personality and Social Psychology, 64, 678-691.

Weaver, A. J., Pargament, K. I., Flannelly, K. J., \& Oppenheimer, J. E. (2006). Trends in the scientific study of religion, spirituality, and health: 1965-2000. Journal of Religion and Health, 45, 208-214.

Webb, P. R. (1977). Voluntary social welfare service A quarter-century of Hong Kong : Chung Chi College 25th anniversary symposium. Hong Kong: Chinese University of Hong Kong. Chung Chi College.

Weber, M. (1958). The Protestant ethic and the spirit of capitalism. New York: Charles Scribner's Sons.

Weick, K. E. (1979). The social psychology of organizing (2d ed.). Reading, Mass.: AddisonWesley Pub. Co.

Weisner, T. S. (1996). Why Ethnography should be the most important method in the study of human development. In R. Jessor, A. Colby \& R. A. Shweder (Eds.), Ethnography and human development : Context and meaning in social inquiry. Chicago: University of Chicago Press.

Welch, M. R., Sikkink, D., Sartain, E., \& Bond, C. (2004). Trust in God and trust in man: The ambivalent role of religion in shaping dimensions of social trust. Journal for the Scientific study of religion, 43(3), 317-343.

Whitford, H. S., \& Olver, I. N. (2009). The multidimensionality of spirituality wellbeing: Meaning, peace, and faith and their association with quality of life and coping in an oncological population. Paper presented at the 3rd Australian Conference on Spirituality and Health: Integrating spirituality in the practice of health care., Adelaide 13-15 July 2009.

Whiting, B. B. (1980). Culture and social behavior: A model for the development of social behavior. Ethos, 8, 95-116.

Whiting, B. B., Whiting, J. W. M., \& Longabaugh, R. (1975). Children of six cultures: A psycho-cultural analysis. Cambridge, Mass.: Harvard University Press.

Wicker, A. W. (1991). Behavior settings reconsidered: Temporal stages, resources, internal dynamics, context. In D. Stokols \& I. Altman (Eds.), Handbook of environmental psychology. Malabar, Fla.: Krieger Pub. Co.

Wicker, A. W., McGrath, J. E., \& Armstrong, G. E. (1972). Organization size and behaviour setting capacity as determinants of member participation. Behavioural Science, 17, 499-513.

Wiebe, D. (1999). Does Understanding Religion require religious understanding? In R. T. McCutcheon (Ed.), The insider/outsider problem in the study of religion: a reader. London ; New York: Cassell.

Wiken, P. H. (1971). Size of organizations and member participation in church congregations. Administrative Science Quarterly, 16(2), 173-179.

Wilkinson, R. G. (1996). Unhealthy societies: the afflictions of inequality. London ; New York: Routledge.

Williams, D. G. (1988). Gender, marriage, and psychosocial well-being. Journal of Family Issues, 9(4), 452-468. 
Wilson, W. (1967). Correlates of avowed happiness. Psychological Bulletin, 67(4), 294-306. Wong, T. W. P. (2003). Colonial governance and the Hong Kong story. In N. Pun \& L. Yu (Eds.), Narrating Hong Kong culture and identity. Hong Kong: Oxford University Press.

Wong, Y. M. (2001). The conceptual changes of social responsibility in the 1950s and 1960s and their corresponding undertakings (Chinese). Jian Dao, 15, 393-430.

Wood, R. L. (1999). Religious culture and political action. Sociological Theory, 17(3), 307332.

Woodberry, R. D. (2003). Researching spiritual capital: Promises and pitfalls. Retrieved June 112006 from http://www.spiritualcapitalresearchprogram.com/pdf/woodberry.pdf.

Wu, D. C. W., \& Fok, A. O. K. (2006). Growth amidst changes: Studies on Hong Kong church 2006. Hong Kong: Hong Kong Church Renewal Movement Ltd.

Wulff, D. M. (1997). Psychology of religion : classic and contemporary (2nd ed.). New York: John Wiley \& Sons.

Wuthnow, R. (1991). Acts of compassion: Caring for others and helping ourselves. Princeton, N.J.: Princeton University Press.

Wuthnow, R. (1999). Mobilizing civic engagement: The changing impact of religious involvement. In M. Fiorina \& T. Skocpol (Eds.), Civic engagement in American democracy. Washington, DC: Brookings Institution.

Wuthnow, R. (2004a). Presidential address 2003: The challenge of diversity. Journal of the Scientific Study of Religion, 43(2), 159-170.

Wuthnow, R. (2004b). The religious factor revisited. Sociological Theory, 22(2), 205-218.

Yardley, L., \& Marks, D. F. (2004). Research methods for clinical and health psychology. London: SAGE.

Yin, R. K. (1994). Case study research: Design and methods (2nd ed. ed.). Thousand Oaks, CA: Sage.

Yin, R. K. (2003). Case study research: Design and methods (3rd ed.). Thousand Oaks, CA.: Sage Publications.

Ying, F. T. (2004). Introduction to Christian church history of Hong Kong. Hong Kong: Alliance Bible Seminary.

Zullig, K. J., Ward, R. M., \& Horn, T. (2006). The association between perceived spirituality, religiosity, and life satisfaction: The mediating role of self-rated health. Social Indicators Research, 79, 255-274. 


\section{Appendix 1: Spirituality and Well-being Survey}

$\underline{\text { Demographic Variables }}$

\begin{tabular}{|c|c|c|c|c|c|c|c|}
\hline Sex: & \multicolumn{3}{|l|}{$\square$ Male } & \multicolumn{4}{|c|}{$\square$ Female } \\
\hline \multicolumn{8}{|l|}{ Age: } \\
\hline \multirow[t]{2}{*}{ Education } & \multicolumn{2}{|l|}{$\square$ Primary } & \multicolumn{3}{|c|}{$\square$ Junior Secondary } & \multicolumn{2}{|c|}{$\square$ Senior Secondary } \\
\hline & \multicolumn{2}{|c|}{$\square$ Tertiary education } & \multicolumn{2}{|c|}{$\square$ University } & & \multicolumn{2}{|c|}{$\square$ Postgraduate } \\
\hline Marital Status & $\square$ Single & \multicolumn{2}{|l|}{$\square$ Married } & $\square$ Cohabit & & eparated & $\square$ Widow \\
\hline \multicolumn{8}{|l|}{ Number of children } \\
\hline Employment Status & $\square$ Employed & \multicolumn{2}{|c|}{$\square$ PT/ Casual } & $\square$ Unemployed & \multicolumn{2}{|c|}{$\square$ Housewife } & $\square$ student \\
\hline \multirow[t]{2}{*}{ Working nature } & $\square$ Student & \multicolumn{2}{|c|}{$\begin{array}{l}\square \\
\text { Professional }\end{array}$} & $\begin{array}{l}\square \text { Managerial } \\
\text { level }\end{array}$ & \multicolumn{2}{|c|}{$\square$ Clerical } & $\square$ Blue collar \\
\hline & \multicolumn{3}{|c|}{$\square$ customer service } & \multicolumn{4}{|c|}{$\square$ Others, please specify } \\
\hline \multirow[t]{2}{*}{ Monthly Income } & $\square<\$ 5000$ & \multicolumn{2}{|l|}{$\begin{array}{l}\square \$ 5000- \\
10000\end{array}$} & $\begin{array}{l}\square \\
20000\end{array} \$ 10000-$ & & 00 & $\begin{array}{l}\square \quad \$ 30000- \\
40000\end{array}$ \\
\hline & $\square>\$ 40000$ & \multicolumn{2}{|l|}{$\square$ NA } & & & & \\
\hline
\end{tabular}

1. Below are five statements that you may agree or disagree with. Please indicate your agreement with each item using the following scale. (Satisfaction with life scale)

\begin{tabular}{|c|c|c|c|c|c|}
\hline $\begin{array}{l}\text { Strongly } \\
\text { Disagree }\end{array}$ & Disagree & $\begin{array}{l}\text { Slightly } \\
\text { disagree }\end{array}$ & $\begin{array}{l}\text { Neither } \\
\text { agree or } \\
\text { disagree }\end{array}$ & $\begin{array}{c}\text { Slightly } \\
\text { agree }\end{array}$ & Agree \\
\hline
\end{tabular}

1) In most ways my life is close to my ideal

2) The conditions of my life are excellent

3) I am satisfied with my life

4) So far I have gotten the important things I want in my life

5) If I could live my life over, I would change almost nothing. 
2. Below are the statements that you may agree or disagree with. Please indicate your agreement with each item using the following scale.

\begin{tabular}{|c|c|c|c|}
\hline $\begin{array}{l}\text { Strongly } \\
\text { Disagree }\end{array}$ & Disagree & $\begin{array}{l}\text { Neither } \\
\text { agree or } \\
\text { disagree }\end{array}$ & Agree \\
\hline
\end{tabular}

1) Generally speaking, most people can be trusted.

2) Your co-worker can best be described as honest

3) In general your neighbours are just looking out for themselves

4) Most people would try to take advantage of you

5) Your neighbours try to take advantage of others

6) Most of the time people try to be helpful

7) Your co-workers try to take advantage of others

8) In general your co-workers are just looking out for themselves

9) Your neighbours can best be described as honest

3. How many voluntary associations, other than your church/ NGO, do you belong to or affiliated with?

4. Please tick the box (es) of the associations that you belong to or affiliated with:

$\begin{array}{lll}\square \text { Social service groups } & \square \text { Professional associations } & \square \text { Environmental groups } \\ \square \text { Youth work } & \square \text { Community based associations } & \square \text { Health groups } \\ \square \text { Recreation groups } & \square \text { Senior citizens groups } & \square \text { Women's group } \\ \square \text { Political groups } & \square \text { Education, arts or culture groups } & \square \text { Support Groups } \\ \square \text { None } & \square \text { Other, please specify: }\end{array}$

5. In the past 4 years, have you joined the following activities?
Activities
Never
Seldom
Sometimes
Often Always

Volunteering in some organizations

Served on any committee in some organizations

Vote in the election (e.g. Legislative Council or District Council)

Reading newspaper

Follow the current affair/ political news 


\section{For each of the following questions, please select the response that best describes your answer.}

i. In general, would you say your health is:
Excellent
Very good
Good
Fair
Poor

ii. The following questions are about activities you might do during a typical day. Does your health now limit you in these activities? If so, how much?

a. Moderate activities, such as moving a table, pushing a vacuum cleaner, bowling, or playing golf

b. Climbing several flights of stairs

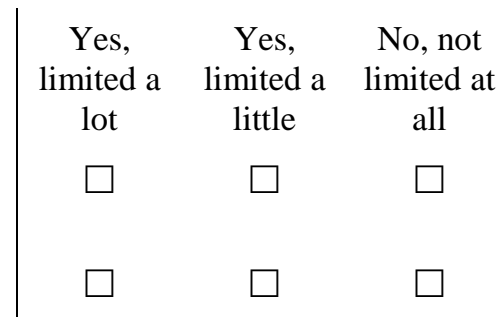

iii. During the past 4 weeks, how much of the time have you had any of the following problems with your work or other regular daily activities as a result of your physical health?

$\begin{array}{cccc}\begin{array}{c}\text { All of the } \\ \text { time }\end{array} \text { the time } & \begin{array}{c}\text { Some of } \\ \text { the time }\end{array} & \begin{array}{c}\text { A little of } \\ \text { the time }\end{array} & \begin{array}{c}\text { None of } \\ \text { the time }\end{array}\end{array}$

a. Accomplished less than you would like

b. Were limited in the kind of work or other activities

iv.. During the past 4 weeks, how much of the time have you had any of the following problems with your work or other regular daily activities as a result of any emotional problems (such as feeling depressed or anxious)?

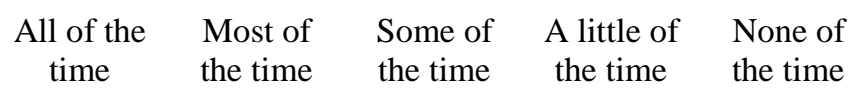

a. Accomplished less than you would like

b. Did work or other activities less carefully than usual

v. During the past 4 weeks, how much did pain interfere with your normal work (including both work outside the home and housework)?
Not at all
A little bit
Moderately
Quite a bit
Extremely

vi. These questions are about how you feel and how things have been with you during the past 4 weeks. For each question, please give the one answer that comes closest to the way you have been feeling. How much of the time

during the past 4 weeks.

\begin{tabular}{|c|c|}
\hline $\begin{array}{l}\text { All of the } \\
\text { time }\end{array}$ & $\begin{array}{l}\text { Most of } \\
\text { the time }\end{array}$ \\
\hline
\end{tabular}
a. Have you felt calm and peaceful?
b. Did you have a lot of energy?
c. Have you felt downhearted and depressed?

vii. During the past 4 weeks, how much of the time has your physical health or emotional problems interfered with your social activities (like visiting friends, relatives, etc.)? 
All of the time $\quad$ Most of the time Some of the time A little of the time None of the time

7. Below are some statements that you may agree or disagree with. Please indicate your agreement with each item using the following scale

\section{Statements}

Strongly

Disagree

1) Current losses are not necessarily bad for one's long-term future.

2) People may have opposite behaviours on different occasions.

3) Kind-hearted people usually suffer losses.

4) There are many ways for people to predict what will happen in the future.

5) There is a supreme being controlling the universe.

6) Power and status make people arrogant.

7) Powerful people tend to exploit others.

8) Fate determines one's successes and failures.

9) Most disasters can be predicted.

10) One has to deal with matters according to the specific circumstances.

11) Human behavior changes with the social context.

12) People will stop working hard after they secure a comfortable life

13) Religion makes people escape from reality.

14) There are certain ways to help us improve our luck and avoid unlucky things.

15) Adversity can be overcome by effort.

16) Every problem has a solution.

17) Belief in a religion helps one understand the meaning of life.

18) One will succeed if he/she really tries.

19) Kind-hearted people are easily bullied.

20) Knowledge is necessary for success.

21) Individual characteristics, such as appearance and birthday, affect one's fate.

22) Belief in a religion makes people good citizens.

23) Hard working people will achieve more in the end.

24) Religious faith contributes to good mental health.

25) One's behaviors may be contrary to his or her true feelings.

\begin{tabular}{|c|c|c|}
\hline Disagree & $\begin{array}{l}\text { Neither } \\
\text { agree or } \\
\text { disagree }\end{array}$ & Agree \\
\hline
\end{tabular}


8. Please think about of being a member of your church/ NGO, respond to the following statements on the basis of how you feel.

1) I think $X X X^{18}$ is a good place for me to be

2) People at $X X X$ do not share the same values

3) Other members and I want the same things from XXX

4) I can recognize most of the people who belong to $\mathrm{XXX}$

5) I feel at home at $X X X$.

6) Very few people at XXX know me.

7) I care about what other members of XXX think of my

Strongly
Disagree

Disagree

Agree Strongly

Agree actions.

8) I have no influence over what XXX is like.

9) If there is a problem in XXX people who come from here can get it solved.

10) It is very important to me to belong to this particular church

11) People at XXX generally don't get along with each other

12) I expect to belong to $\mathrm{XXX}$ for a long time

9. The following identity is important to me

1) the member of $X X X$ congregation/group of NGO

do not

Completely agree at all

2) the member of $X X X$ denomination / NGO

3) Christian*

4) Hong Konger

5) Chinese

10. To what extent do you think your church/ group

1

2
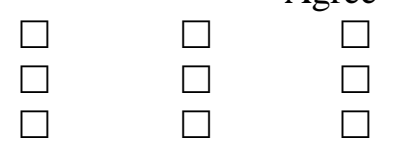
11. Compared with the other church/ NGO, what do you think are the major 3 characteristics of your church/ NGO?

12. What do you think are the goals of your church/ NGO?

Measure on Religion and Spirituality

13. What is your religious belief?

$\square$ Protestant

None

Others, please specify:

14. The following includes items which you may have or may not experienced, please consider how often you directly have this experience, and try to disregard whether you feel you should or should not have these experiences. A number of items use the word "God". If this word is not a comfortable one for you, please substitute another idea which calls to mind the divine or holy for you.

$\begin{array}{cccccc}\begin{array}{c}\text { Many } \\ \text { times a } \\ \text { day }\end{array} & \begin{array}{c}\text { Every } \\ \text { day }\end{array} & \begin{array}{c}\text { Most } \\ \text { days }\end{array} & \begin{array}{c}\text { Some } \\ \text { days }\end{array} & \begin{array}{c}\text { Once in } \\ \text { a while }\end{array} & \begin{array}{c}\text { Never } \\ \text { or } \\ \text { almost }\end{array}\end{array}$

1) I feel God's presence

2) I experience a connection to all life.

3) During worship, or at other times when connecting with God, I feel joy which lifts me out of my daily concern

4) I find strength in my religion or spirituality

5) I find comfort in my religion or spirituality

6) I feel deep inner peace or harmony

7) I ask for God's help in the midst of daily activities

8) I feel guided by God in the midst of daily activities

9) I feel God's love for me, directly

10) I feel God's love for me, through others

11) I am spiritually touched by the beauty of creation

12) I feel thankful for my blessings

13) I feel a selfless caring for others

14) I accept others even when they do things I think are wrong.

15) I desire to be closer to God or in union with the divine

16) In general, how close do you feel to God? Not at all
close $\quad \begin{gathered}\text { As close as } \\ \text { possible }\end{gathered}$

15. This survey is part of an investigation of general public opinion concerning a variety of social issues. You will probably find that you agree with some of the statements, and disagree with 
others, to varying extents. Please indicate your opinion by giving a tick in the relevant boxes, according to the following scale:

-4 if you very strongly disagree with the statement

-3 if you strongly disagree with the statement

-2 if you moderately disagree with the statement

-1 if you slightly disagree with the statement

0 if you are neutral about the statement
4 if you very strongly agree with the statement 3 if you strongly agree with the statement 2 if you moderately agree with the statement 1 if you slightly agree with the statement

1) God has given humanity a complete unfailing guide to happiness and salvation, which must be totally followed

2) No single book of religious teachings contains all the intrinsic, fundamental truths about life

3) The basic cause of evil in this world is Satan, who is still constantly and ferociously fighting against God

4) It is more important to be a good person than to believe in God and the right religion

5) There is a particular set of religious teachings in this world that are so true, you can't go any deeper because they are the basic, bedrock message that God has given humanity.

6) When you get right down to it, there are basically only two kinds of people in the world: the Righteous, who will be rewarded by God, and the rest, who will not.

7) Scriptures may contain general truths, but they should not be considered completely literally true from beginning to end

8) To lead the best, most meaningful life, one must belong to the one, fundamentally true religion

9) "Satan" is just the name people give to their own bad impulses. There really is no such thing as a diabolical "Prince of Darkness" who tempts us.

10) Whenever science and sacred scripture conflict, science is probably right

11)The fundamentals of God's religion should never be tampered with, or compromised with others' beliefs

12) All of the religions in the world have flaws and wrong teachings. There is not perfectly true, right religion.

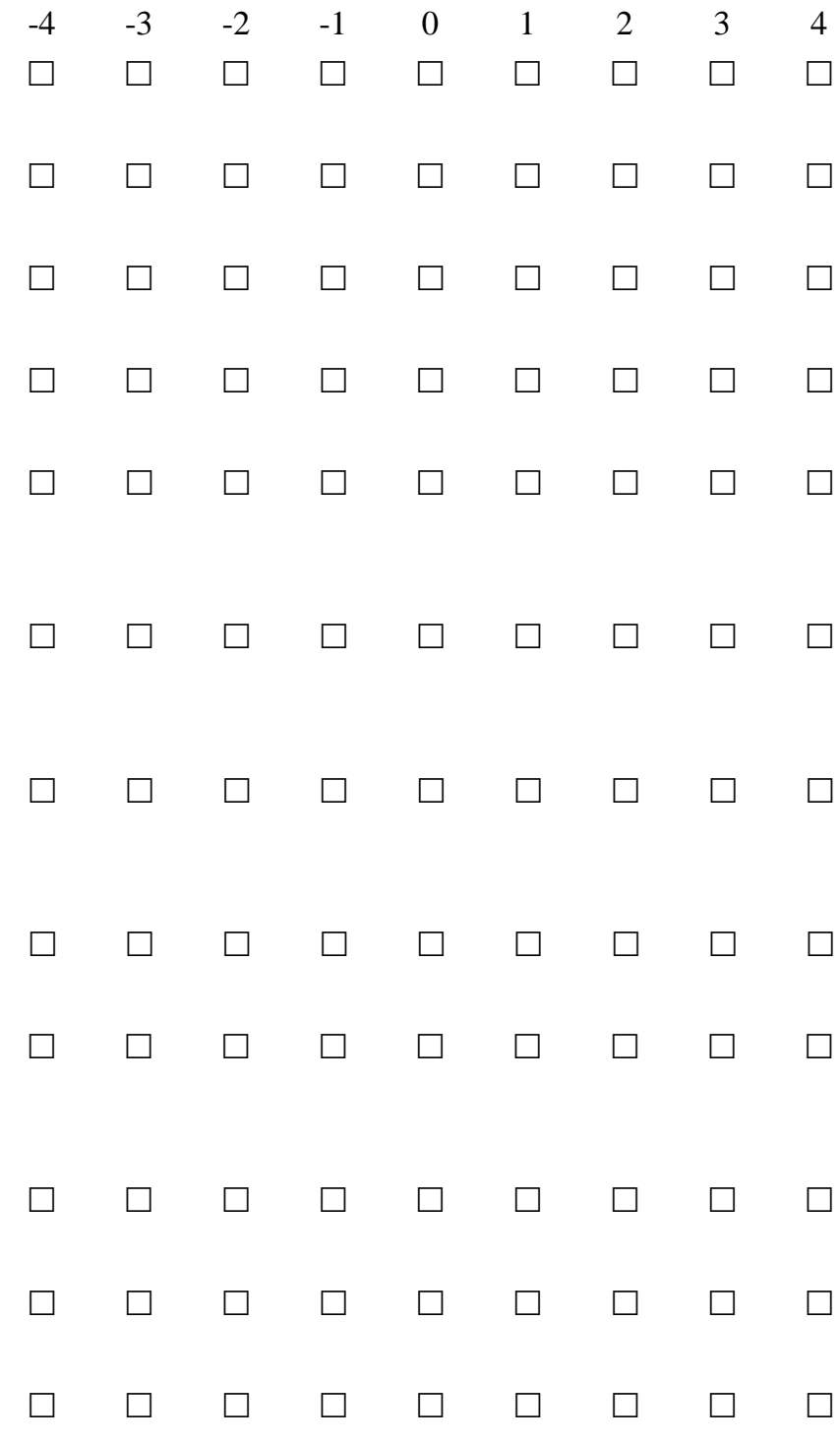


16. How often do you participate in the followings?

\begin{tabular}{|c|c|c|c|c|}
\hline $\begin{array}{l}\text { Daily } \\
\text { or } \\
\text { more }\end{array}$ & $\begin{array}{c}\text { Weekly } \\
\text { or } \\
\text { more }\end{array}$ & $\begin{array}{c}2-3 \\
\text { times a } \\
\text { month }\end{array}$ & $\begin{array}{l}\text { Once a } \\
\text { month }\end{array}$ & $\begin{array}{l}\text { Few } \\
\text { times a } \\
\text { year }\end{array}$ \\
\hline
\end{tabular}

1) Private prayer \& meditation

2) Bible reading

3) Serve in church mission

17. How many years have you been coming to worship services and/ or activities run by the church that you attended? Not applicable

18. How often do you attend worship services at your church

$\square$ Weekly

$\square$ Fortnightly

Once a month

Less than once a month

Special occasions

Never

19. Apart from worship services, how many hours a month do you spend in committees, social events, educational and outreach programs of this church in average?

hours a month

20. Have you taken part in any of the following activities organized by your church? If so, please put a tick in the box next to the activity, and indicate roughly the time you have spent on it in an average month.(Measure on congregation involvement)

1) Fellowship

2) Nurturing new believer program

3) Personal evangelical preaching

4) Missionary

5) Church planting/ church development

6) Sunday bible school teaching

7) The mentor or leader in the fellowship

8) Singsparation / Worship team training

9) Choir

10) Service leader

11) Prayer team

12) Meditation training

13) Visitation

14) Social concern

15) Church Leadership training/ meeting

16) Discipleship training/ Bible study approximately

approximately approximately approximately approximately approximately approximately approximately approximately approximately approximately approximately approximately approximately approximately approximately hours a month hours a month hours a month hours a month hours a month hours a month hours a month hours a month hours a month hours a month hours a month hours a month hours a month hours a month hours a month hours a month 
17) Group Leader training/ meeting

18) Theology training

19) Counseling

20) Spiritual healing

21) Others, please specify: approximately __ hours a month

approximately hours a month

approximately hours a month

approximately hours a month

approximately hours a month

Thank you very much for completing this survey 


\section{Appendix 2}

\section{Assessing Model Invariance between the five settings}

To assess if the tested path model (Figure 11 Path Model C) also operates in the five studied spiritual settings, multiple group path analysis was conducted. Nevertheless, as explained before, the interpretation should be treated with great caution because of the limitation in the sample size of each setting.

Assessing model invariance between groups is normally conducted in two phases (Meyers et al., 2006). In the first phase, an "unconstrained” or free model, in which the path coefficient will be estimated simultaneously and separately for each group, will be compared with a "constrained" or null model, in which the corresponding path coefficient of each group was supposed to be equal to the other. Two chi-square fit measures will respectively show whether the model, either the unconstrained or constrained model, fits the data. Then a chisquare goodness of fit test will determine if there is a significant difference between the fit measures for the two models. Once differences between groups are identified, we will enter the second phase. Each pair of estimated path coefficients will be compared to see if differences exist across the various settings.

The chi-square test for differences revealed that the hypothesized model was not equally applied to the five settings, $\Delta \chi^{2}(56)=99.43, \mathrm{p}<.001$. The NFI, CFI and RMSEA findings further demonstrate that the free model has a better fit with the data than the fixed model. It seems to suggest that different groups have different path coefficients for the hypothesized model of well-being. The estimated path coefficient for each setting, suggested by the free model, is provided in the following figures.

Table

Fit indices across Endogenous Variables, and Chi-Square difference Tests for the Models

\begin{tabular}{lllllllll}
\hline Model & $\chi^{2}(\mathrm{df})$ & $\mathrm{p}$ & $\mathrm{NFI}$ & $\mathrm{CFI}$ & $\mathrm{TLI}$ & RMSEA & $\chi^{2}$ difference $(\mathrm{df}=1)$ & $\mathrm{P}$ \\
\hline Equal & $217.89(136)$ & .000 & .673 & .832 & .778 & .044 & - & - \\
Free & $118.46(80)$ & .003 & .822 & .921 & .822 & .039 & 99.43 & $<.001$ \\
\hline
\end{tabular}



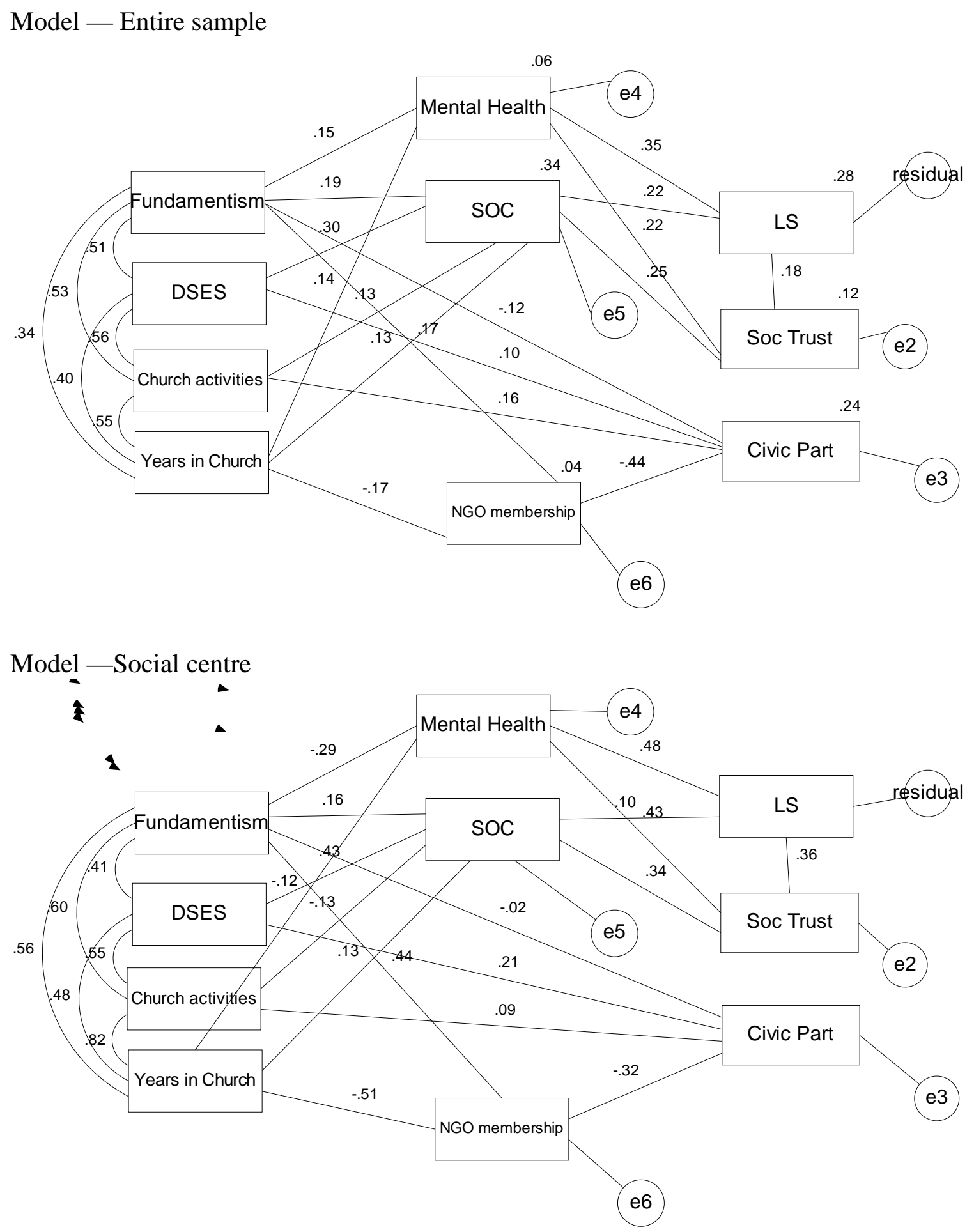

Model - CHC (charismatic) 

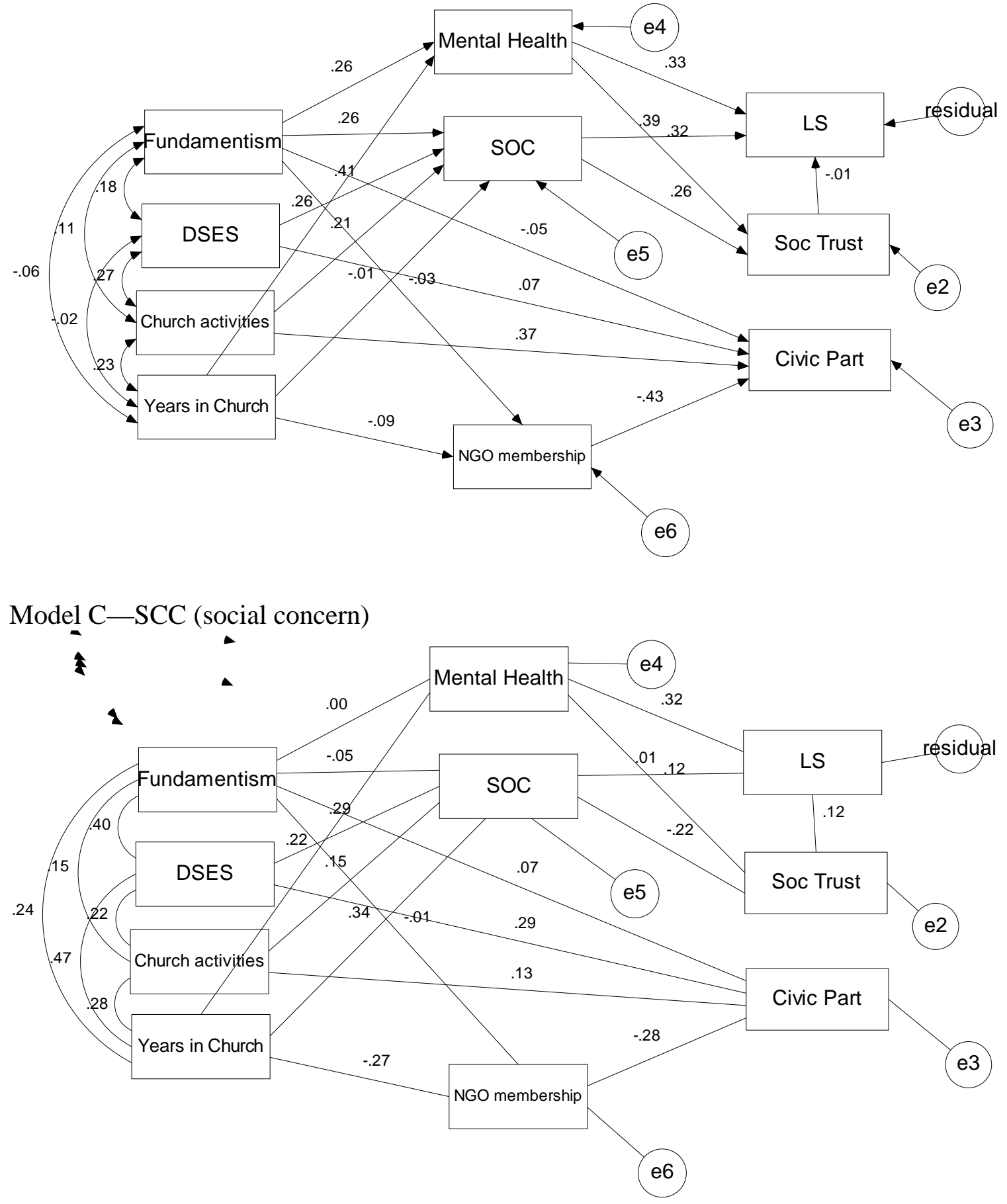

Model C-FDC (conservative) 


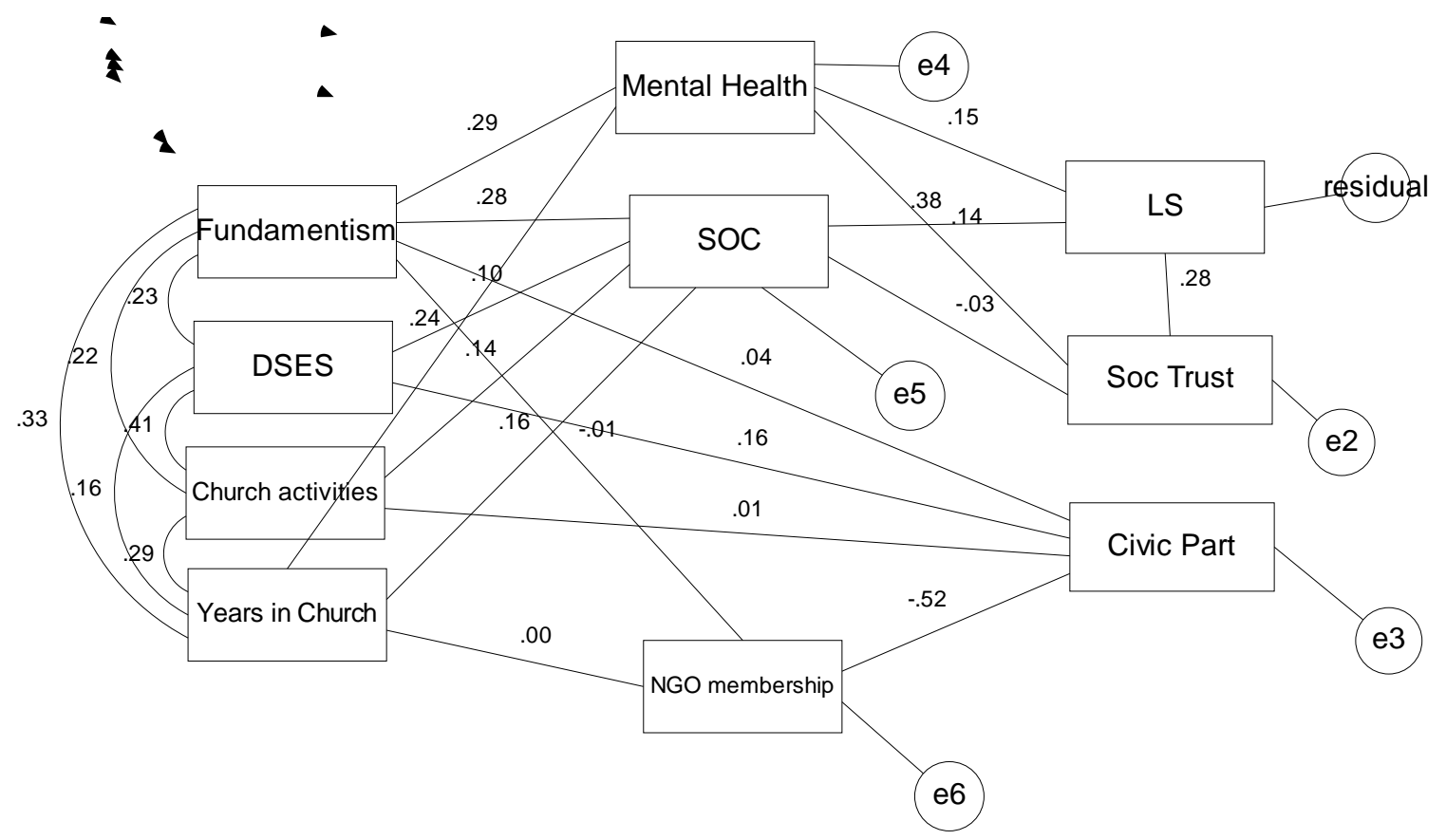

\section{Model C—EVC (evangelical)}

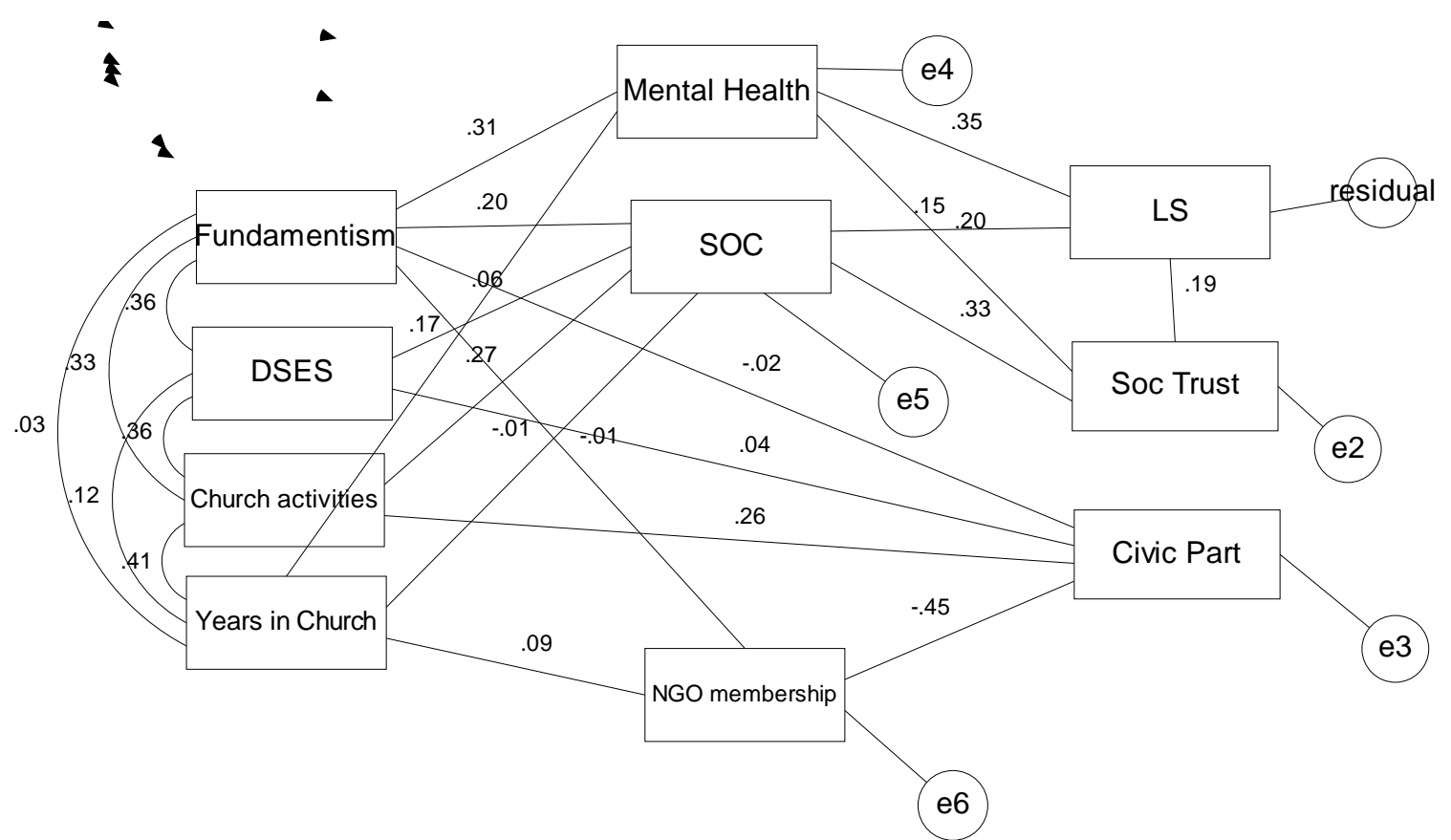




\section{Appendix 3: Interviewee list}

\begin{tabular}{|l|l|l|l|l|l|l|l|l|l|}
\hline \multicolumn{9}{|c|}{ FDC } & \multicolumn{6}{|c|}{ EVC } \\
\hline Person & Sex & Age & C.Y.* & Occupation & Person & Sex & Age & C. Y. & Occupation \\
\hline 1 & F & $\sim 45$ & 21 & Housewife & 1 & F & $25+$ & $\begin{array}{l}9 \\
(20+)^{*}\end{array}$ & Business \\
\hline 2 & F & $50+$ & 40 & Housewife & 2 & M & $25+$ & 0.5 & Technology \\
\hline 3 & M & $25+$ & 20 & $\begin{array}{l}\text { Social } \\
\text { welfare }\end{array}$ & 3 & M & $40+$ & $11(25)$ & Pastoral work \\
\hline 4 & F & 34 & 6 & Education & 4 & F & $\sim 30$ & $10+$ & Business \\
\hline 5 & M & $40+$ & 18 & Government & 5 & F & $\sim 40$ & 28 & Design \\
\hline 6 & M & $\sim 40$ & 12 & $\begin{array}{l}\text { Further } \\
\text { study }\end{array}$ & 6 & & & & \\
\hline
\end{tabular}

\begin{tabular}{|l|l|l|l|l|l|l|l|l|l|}
\hline \multicolumn{9}{|c|}{ SCC } & \multicolumn{6}{|c|}{ CHC } \\
\hline 1 & M & 34 & 24 & $\begin{array}{l}\text { Further } \\
\text { study }\end{array}$ & 1 & M & 34 & 10 & Professional \\
\hline 2 & F & $30+$ & $20+$ & Business & 2 & F & $30+$ & 11 & Business \\
\hline 3 & M & 56 & $\begin{array}{l}40+ \\
(28)\end{array}$ & $\begin{array}{l}\text { Managemen } \\
\text { t }\end{array}$ & 3 & F & $40+$ & $2(30)$ & Housewife \\
\hline 4 & F & $\begin{array}{l}51- \\
55\end{array}$ & $30+$ & Retired & 4 & F & $40+$ & 18 & Government \\
\hline 5 & M & $50+$ & $\sim 30$ & Education & 5 & M & $30+$ & $9(14)$ & Pastoral worker \\
\hline 6 & F & $\sim 30$ & $\sim 10$ & $\begin{array}{l}\text { Social } \\
\text { service }\end{array}$ & 6 & M & $\sim 30$ & $7(12)$ & Design \\
\hline
\end{tabular}

* C. Y. means the number of years the participant attend the studied church. The number in bracket is the number of years of attending church in general (i.e. including the years in the other churches) 


\title{
Appendix 4: Information Letter for Participants
}

\author{
The Study of "Impacts of Spirituality and religious Communities \\ for people in Hong Kong” \\ Student researcher: Eddie Chi Wai Ng; Principal Investigator: Dr. Adrian Fisher
}

Dear Prospective Participant:

I am inviting you to participate in a research study that I am conducting for my $\mathrm{PhD}$ thesis in Psychology at Victoria University, under the supervision of Dr. Adrian Fisher. The purpose of this study is to discover what the qualities and impacts of spirituality and faith-communities are for people. I am now recruiting for around 5 to10 participants who can share with me their spiritual life and church life. The interview will be tape-recorded so that the interview can later be transcribed and coded for themes.

The interview will take about 1-2 hours, with breaks if necessary. Around two month following the interview, the transcribed interview and summarized result will be shown to the participants, so that feedback on the themes drawn from the interview can be obtained.

Please note that your participation in this research is purely voluntary, and that if you choose not to participate that this will not in any way affect your relationship with the church or your participation in any activities or programs. You are under absolutely no obligation to participate in this research.

The only risk that we anticipate is that some of the questions may trigger experiences that caused you distress. Please note that we want to make the interview as comfortable as possible for you. Moreover, you are free to refuse to answer any question without penalty. If you leave the interview before data collection is completed, your responses will be destroyed and omitted from the transcripts.

Everything you say during the interview will be held in the strictest confidence by the researchers. Your name will not be associated with anything you say and your interview responses will be securely stored. Nevertheless, due to the nature of the study it will be necessary to use quotations from participants in the final report of the findings. No quotes will contain information that will jeopardize the anonymity of participants (i.e., quotes will not identify who you are). If you do not want any quotations to be written into the final report you may inform the researcher of this. The accuracy of quotations will be verified with participants before being added to the final document. You may choose to be identified (not be anonymous) in the research project. In addition, the results of the research may be presented at some psychology conference. The final report will be submitted to the Victoria University School of Psychology and will remain on file there.

If you have questions at any time about the research or the procedures, or you experience adverse effects as a result of participating in this study, you may contact either of us, Eddie Ng, 94888671, or Dr. Adrian Fisher, (613) 99195221, or through email Adrian.fisher@vu.edu.au. Any queries or complaints about the way you have been treated, you may contact the Secretary, Victoria University Human Research Ethics Committee, Victoria University, PO Box 14428, Melbourne VIC 8001, Australia. Telephone +613-9919-4710

Sincerely yours,

Eddie Ng, School of Psychology

Victoria University 


\section{Need to talk to someone?}

It is not unusual for people to experience periods of loneliness, confusion or struggle in terms of their life situation.

Below are the contact numbers of the counseling service of some voluntary organizations in Hong Kong that may be helpful.

\begin{tabular}{|l|l|l|}
\hline Organization & Contact numbers & Website \\
\hline $\begin{array}{l}\text { Hong Kong } \\
\text { Christian Service }\end{array}$ & 27316227 & http://www.hkcs.org/fcb/cp/cp.htm \\
\hline Breakthrough & $2377-8511$ & http://www.breakthrough.org.hk \\
\hline YWCA & 34431601 & http://www.ywca.org.hk/hotline/main.htm \\
\hline $\begin{array}{l}\text { Hong Kong Mood } \\
\text { Disorders Center }\end{array}$ & 28330838 & http://www.hmdc.med.cuhk.edu.hk/main.html \\
\hline
\end{tabular}




\section{Appendix 5: Consent Form for Participants}

The Impacts of Protestant Spirituality and Religious Communities on the well-being of People in Hong Kong

Student Researcher: Eddie, Chi Wai Ng; Principal Investigator: Dr. Adrian Fisher;

School of Psychology, Victoria University.

I have been informed of the purposes and methods of the above named study and what will be required of me to participate in this study. $\quad$ Furthermore, I understand that:

+ I am free to withdraw my participation at any time and to not answer any questions which I do not wish to answer;

+ The researchers will provide me with the complete transcript of the interview, if I wish, which I can review and return to the researchers with my comments;

+ The transcript of my interview will be stored in a locked cabinet to protect my confidentiality;

+ My name will not be associated with any quotes; Unless I request so;

Participant’s Signature

Researcher's Signature

I have read and understand the information from the informed consent letter. I agree to participate in the focus group for this research.

Yes__ No

I agree to have the interview tape-recorded.

Yes

No

I understand that quotes of things that I say may appear in reports or presentations available to the public, but only in an anonymous form, so that I cannot be identified as the source of these quotes.

Yes

No 


\section{Appendix 6: Interview Guide}

Research in the area of religion, spirituality, and community psychology has suggested that there are great impacts on the people's psychological health of one's spiritual practices and being a member of a faith community. The purpose of this interview is to determine how your personal spirituality and membership in the congregation (at XXX church) has impacted your life.

1) Background information

- How long do you believe in Christian faith?

- How long do you join this church?

2) Personal faith and spiritual practices

- Tell me about your personal faith (probe for beliefs and values concerning God, world and society, human nature, self).

- Why you believe in this religious faith?

- Can you share some of the important moments in your spiritual life?

3) Description of the faith community

- Tell me about the congregation here

- Can you share some of the important moments in your church life?

- What are some common values shared between members within this community?

- What is it like here?

- Are there any legacies or stories widely circulated in your church? Can you describe?

- In what ways do you participate at your church?

- Why you join this congregation? Why do you continue to be involved?

- What aspects of your church did you cherish the most?

- Does this community provide opportunities for participation? Describe some of them and that you have participated.

- Does this community give members a sense of belonging? Describe some of the examples

- What resources and activities are available from your church

- Does your church have a strong or distinguished identity? Can you describe what is it about?

5) Well-being

- What is a good life? What is life satisfaction?

6) Well-being outcomes of one's faith and spiritual practices

- In what ways has your life changed as a result of your faith? How has the spiritual practices you perform changed your life, your relation with others and your relation with the society?

7) Well-being outcomes of belonging to this faith community 
- How has your life changed as a result of belonging to this faith community? How does your belonging change the way you feel about yourself, your relation with others and your relation with the society?

Is there anything you would like to add that has not already been mentioned? Thank-you for participating in this interview. I will get back to you in a 2 months to go over quotes and my interpretations of them. 


\section{Appendix 7: Social concern church activities}

The programs in SCC can be divided into three major categories: educational or awareness raising, action and supportive. The following are just some of the examples of the educational type programs:

1. Seminars or forums on Christian and the Basic Law (1988), the need of Vietnam refugees in local community (1988), Chinese identity (1994), Crisis in Kosovo (1999), educational reform (2000).

2. Study groups on Green Paper on the Further Development of Representative Government in Hong Kong (1987), the Basic Law (1988), and democratic education after June four Tiananmen square incident (1989, 1990).

3. A Study trip to the Philippines was organized in 1989 to learn how the churches there respond to societal need and development. Summer camp about Christianity and student movement in China was organized in 1989 for the teenage fellowship.

4. Movie and sharing sessions were arranged so as to broaden the members' horizons towards societal issue or societal need in a mild way. ${ }^{19}$

Besides the educational type program, SCC has also been actively involved in the community and society at large, especially in the past. The programs include initiatives that are either action- oriented or supportive in nature.

1. Community service: SCC has a long tradition serving the needy in the local community by meal delivery, visiting some senior elders in the region on festival day, or organizing some community events promoting health or sense of community.

2. Submission or joint signature in the newspaper: SCC, in the recent decades, often joins signature with other Christian organizations in newspapers expressing their views on and opinions towards societal issues. Besides the annual memorial prayer for the June four (Tiananmen Square) incident published in the newspaper on that day, SCC is also actively engaged in joint signature across wide ranges of societal issues, such as

\footnotetext{
19 For example, in 1989, SCC has screened a famous documentary "He shang”-about the origin of the Chinese civilization--"Yellow River". In 1997, the debatable documentary “The gate of heavenly peace” was screened in the church. In addition, from time to time, SCC also invited some organizations to share the needs of the marginal groups in the society, ranging from homeless, people facing chronical illness to drug addiction.
} 
educational reform, degrading trend of mass media, the abuse of power by the Hong Kong police force, protesting against the Legalisation of soccer gambling and the oppression on the freedom of speech and religious freedom, to name a few.

3. Fundraising or donation: Instead of organizing the donation or fundraising activities themselves, SCC normally supports the works organized by outsides organizations by contributing collectively the money or materials (normally clothes) collected in the church. The recipients of the donation vary widely, from local community (children in the Vietnamese refugee camp in the area), Hong Kong society (patients with cancer or terminal illness, homeless, single parents) to the global world (children in Ethiopia, survivor after the Philippine earthquake, flood victims in eastern China, Kurdish refugee in Iraq).

4. Prayer meeting: Besides the normal prayer meeting, SCC has occasionally organized or co-organized prayer meetings focusing on the societal issues happening locally or globally. For example, SCC had organized prayer meetings regarding the black people living under the South Africa apartheid policies (1985), the establishment of Daya Bay nuclear plant near Hong Kong (1987), the students and activists in the Tiananmen Square protest of 1989, the peace of Hong Kong (1997), and the article 23 of the Basic Law (2003).

5. Other: Besides the actions aforementioned, SCC also provided their church space for some less well known organizations to organize activities, even though some of the activities may be sensitive in nature, such as a seminar talking about the legislation on homosexual issue and activities organized by a parent group of cleft lip and palate. In addition, SCC hosted the accused Korea peasant protestors in 2005 when the WTO conference was held in Hong Kong. SCC also organized visit to refugee camp and the social concern fellowship conducted a small scale survey in 1989 in the community about the people's attitude towards the June Four student movement which happened in China. 